Universidade de São Paulo

Instituto de Astronomia, Geofísica e Ciências Atmosféricas

Departamento de Geofísica

Rodrigo Corrêa Rangel

Inversão Conjunta 2D de Dados de Caminhamento

Elétrico e Caminhamento TDEM Aplicados a Estudos

Hidrogeológicos em Taubaté/SP

São Paulo

2018 

Rodrigo Corrêa Rangel

\title{
Inversão Conjunta 2D de Dados de Caminhamento Elétrico e Caminhamento TDEM Aplicados a Estudos Hidrogeológicos em Taubaté/SP
}

\author{
Versão Corrigida \\ (A versão original encontra-se disponível na Unidade) \\ Dissertação apresentada ao \\ Departamento de Geofísica do Instituto \\ de Astronomia, Geofísica e Ciências \\ Atmosféricas da Universidade de São \\ Paulo como requisito parcial para \\ obtenção do título de Mestre em \\ Ciências. \\ Área de Concentração: Geofísica \\ Orientador: Prof. Dr. Jorge Luís Porsani
}

São Paulo

2018 



\section{Agradecimentos}

Primeiramente agradeço a minha mãe, Maria Conceição Corrêa Rangel, por sempre ter me apoiado nos meus estudos, pois sem seu apoio eu não teria alcançado esse objetivo.

Agradeço a minha namorada, Evelise Cardozo Machado, por sempre ter acreditado no meu potencial e me incentivado a fazer o mestrado. Também agradeço a minha sogra, Tereza Cristina Cardozo Machado, pelo carinho e acolhimento.

Agradeço muito ao meu orientador e amigo, Prof. Dr. Jorge Luis Porsani, por compartilhar o seu conhecimento, acreditar no meu potencial e pela oportunidade de trabalhar nesta pesquisa, a qual incluiu as etapas de aquisição dos dados, processamento dos dados e interpretação dos resultados. Essa experiência me fez adquirir uma perspectiva completa de uma pesquisa científica em geofísica, sendo crucial para o meu crescimento pessoal, acadêmico e profissional.

Agradeço ao amigo Dr. Cassiano Antonio Bortolozo por ter me ensinado a metodologia de Inversão Conjunta 2D CE/CTDEM e compartilhado as suas rotinas no MATLAB, pois seu apoio e voto de confiança foram fundamentais para o desenvolvimento desta pesquisa.

Aos amigos Luiz Rodrigo Hamada, Marco Antonio Couto Junior e Gabriela Serejo de Oliveira por terem compartilhado seus conhecimentos em relação aos métodos elétricos e eletromagnéticos e aos amigos Felipe dos Anjos Neves e Matheus Magalhães Pereira de Andrade pela amizade e companheirismo.

Agradeço aos técnicos de campo e amigos Ernande Costa Santos e Marcelo Cesar Stangari pelas aquisições dos dados e por terem compartilhado as suas experiências comigo, pois esse é um conhecimento extremamente importante na geofísica aplicada.

Agradeço também ao Prof. Dr. Vagner Roberto Elis, ao Prof. Dr. Renato Luiz Prado e à Prof ${ }^{a}$ Dr $^{a}$ Naomi Ussami pelo apoio e incentivo durante a graduação e também no mestrado.

Sou muito grato ao Instituto de Astronomia, Geofísica e Ciências Atmosféricas e à Universidade de São Paulo pela ótima infraestrutura e qualidade de ensino e também à CAPES pela bolsa de estudos.

Por fim, muito obrigado a todos que de alguma forma participaram e contribuíram com a minha formação! 



\section{Sumário}

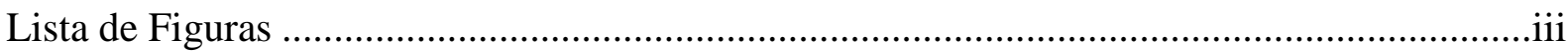

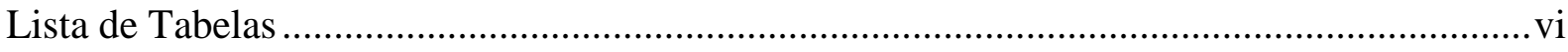

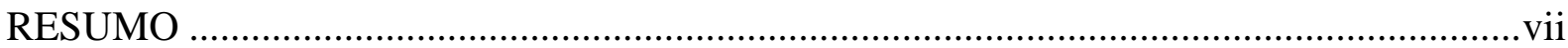

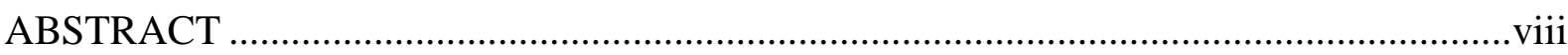

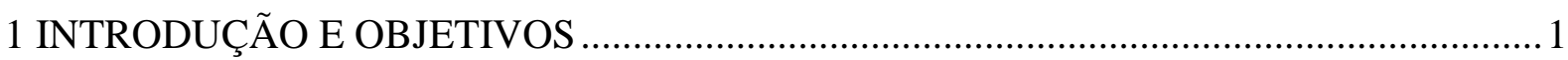

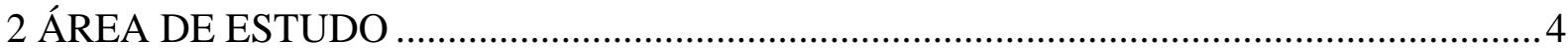

2.1 Aspectos Geológicos da Bacia de Taubaté .................................................................. 4

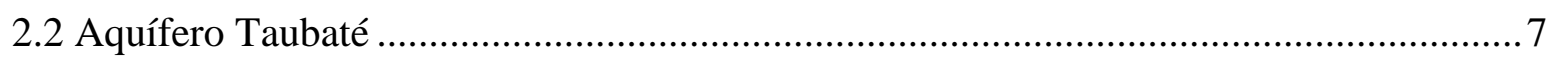

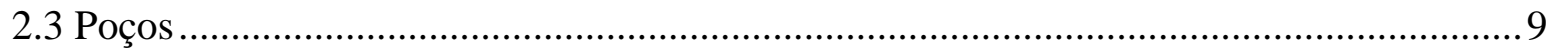

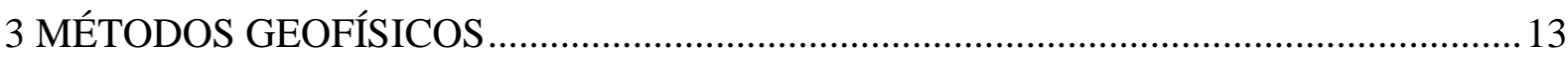

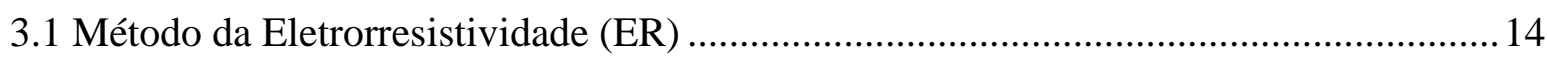

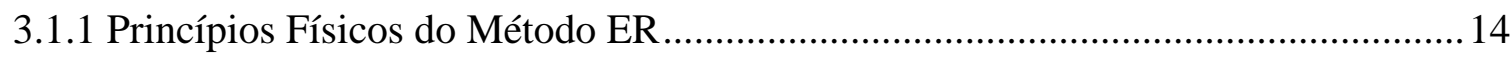

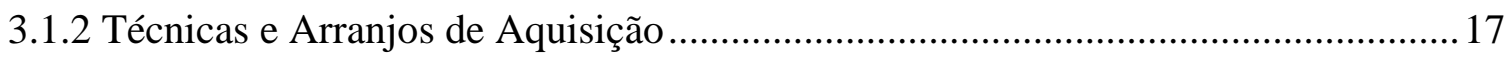

3.2 Método Eletromagnético no Domínio do Tempo (TDEM) ......................................... 19

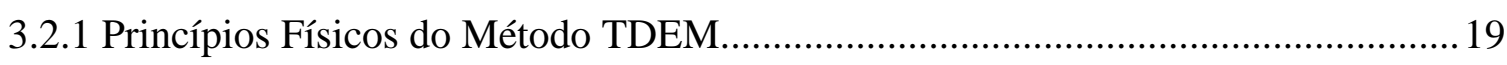

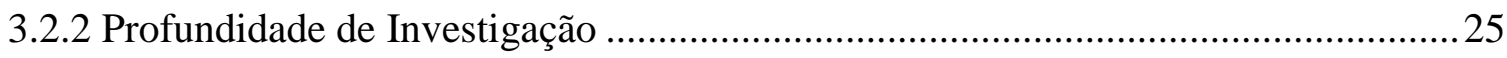

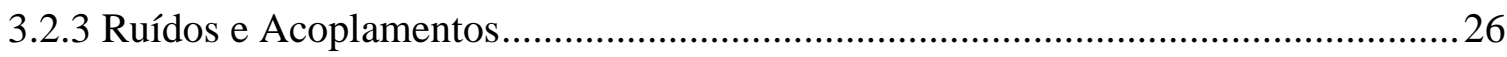

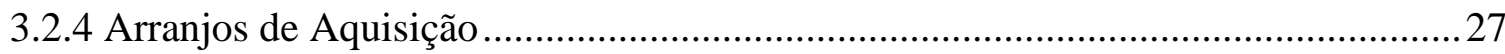

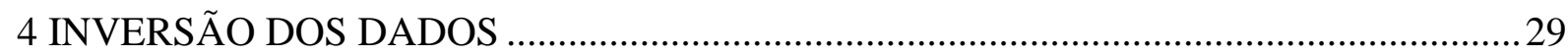

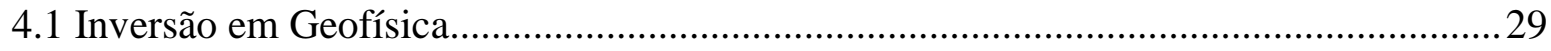

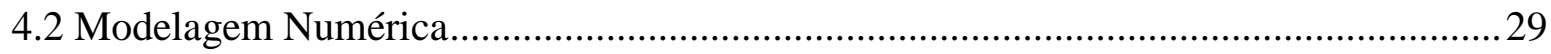

4.2.1 Modelagem ER 2D por Diferenças Finitas ........................................................... 30

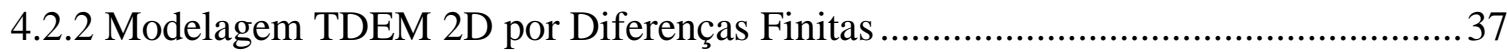

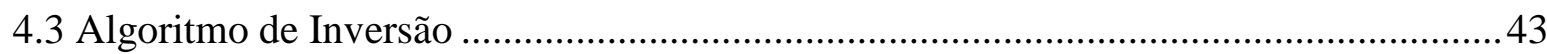




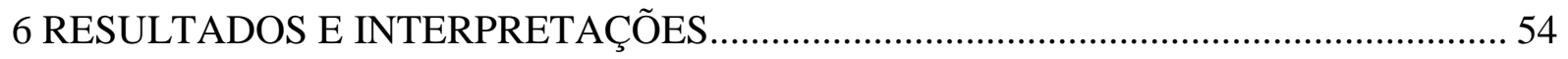

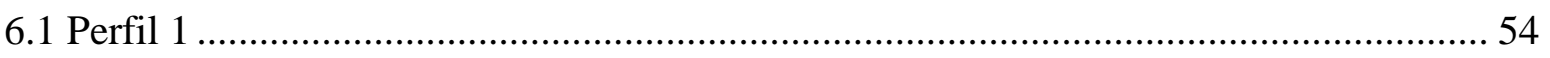

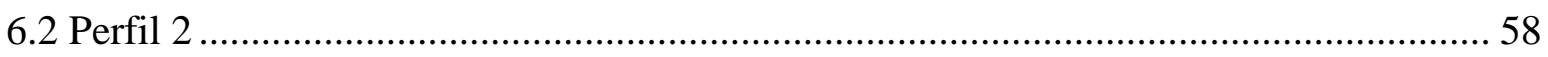

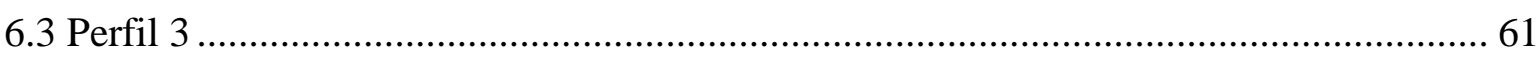

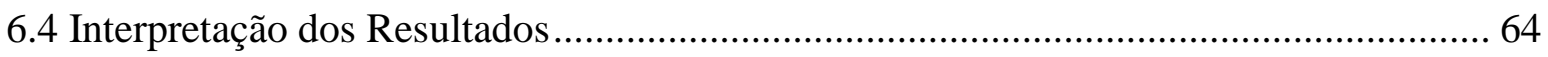

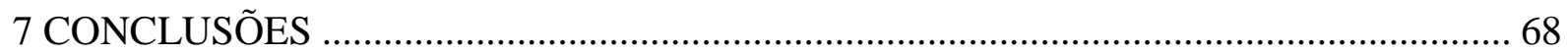

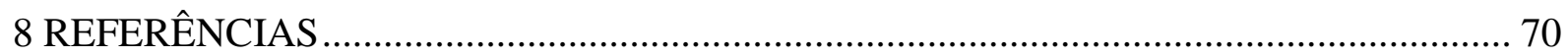

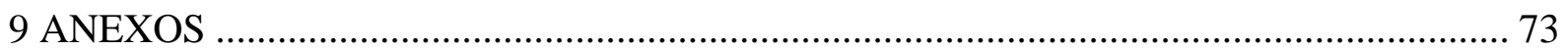

9.1 Resumo expandido para o $15^{\circ}$ Congresso Internacional da Sociedade Brasileira de

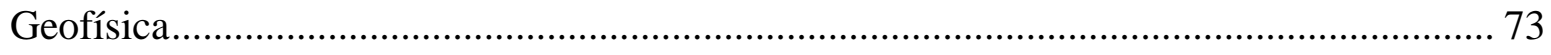

9.2 Artigo publicado pela revista International Journal of Geosciences ............................ 79

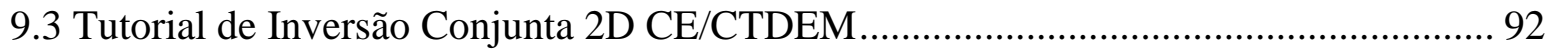




\section{Lista de Figuras}

Figura 1 - Mapa de localização da cidade de Taubaté/SP.

Figura 2 - Localização da Bacia de Taubaté e da área de estudo (modificado de Souza Filho et al., 2013).

Figura 3 - Quadro litoestratigráfico e evolução tectono-sedimentar do segmento central do RCSB - Letras: $\mathrm{p}$ - leques aluviais proximais; $\mathrm{m}-\mathrm{d}$ - leques aluviais medianos a distais associados a planície aluvial de rios entrelaçados; $\mathrm{t}$ - depósitos de tálus; c - depósitos coluviais; ca - depósitos colúvio-aluviais; a - depósitos aluviais (modificado de Riccomini et al., 2004)

Figura 4 - Espessura saturada do Aquífero Taubaté (modificado de DAEE et al., 2005).........8

Figura 5 - Localização dos poços em Taubaté cadastrados no SIAGAS-CPRM e da área de estudo, com destaque para o poço mais profundo (P40).

Figura 6 - Eletrodo pontual na superfície de um meio homogêneo e isotrópico 15

Figura 7 - Arranjo dos eletrodos de corrente $(\mathrm{AB})$ e de potencial $(\mathrm{MN})$ (adaptado de Kearey et al., 2002).

Figura 8 - Sondagem elétrica vertical - arranjo Schlumberger (adaptado de Telford et al., 1990).

Figura 9 - Caminhamento elétrico - arranjo dipolo-dipolo (adaptado de Telford et al., 1990).

Figura 10 - Caminhamento elétrico - arranjo polo-dipolo (adaptado de Telford et al., 1990).18

Figura 11 - Esquema do loop transmissor, bobina receptora e as correntes secundárias induzidas na subsuperfície (adaptado de McNeill, 1994).

Figura 12 - Sequência de eventos do método TDEM (adaptado de Christiansen et al., 2006).

Figura 13 - Fontes de acoplamentos do método TDEM $(\mathrm{R}=$ resistor, $\mathrm{L}=$ indutor e $\mathrm{C}=$ capacitor): a) Acoplamento galvânico; b) Acoplamento capacitivo (adaptado de Sørensen et al., 2000).....

Figura 14 - Arranjos de aquisição TDEM: a) Loop central e b) Caminhamento TDEM com loop transmissor fixo e a bobina receptora móvel.

Figura 15 - Discretização da eletrorresistividade 2D por diferenças finitas. $\Delta \boldsymbol{A} \boldsymbol{i}, \boldsymbol{j}$ representa um elemento de área discretizada (Bortolozo, 2016)................................................. 30

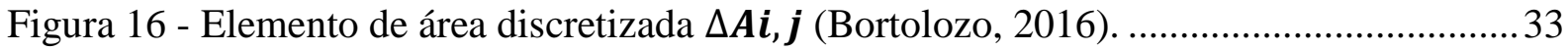

Figura 17 - Exemplo de numeração de nós para uma malha retangular com 16 nós (Bortolozo, 2016). 
Figura 18 - Discretização TDEM 2D por diferenças finitas. Os pontos pretos no ar são necessários para a condição de fronteira na interface Terra-Ar (Bortolozo, 2016)........ 37

Figura 19 - Ponto $\boldsymbol{E} \boldsymbol{i}, \boldsymbol{j}$ na malha de diferenças finitas, cercado por seus vizinhos e elementos de condutividade constante. ABCD é o retângulo formado pela união dos pontos centrais dos quadrados ao redor de $\boldsymbol{E} \boldsymbol{i}, \boldsymbol{j}$ (Bortolozo, 2016).

Figura 20 - Conjunto de soluções possíveis: A para a inversão de um dado de CE, B para a inversão de um dado de CTDEM e C para a inversão conjunta.

Figura 21 - Esquema geral dos arranjos de aquisição de dados ER e TDEM utilizados. Sondagens SEV e TDEM loop central sobrepostas. Quatro perfis de CTDEM e dois perfis de CE nas direções NS e EW.

Figura 22 - Equipamentos utilizados na aquisição dos dados ER: a) Resistivímetro Syscal R2 para SEV e b) Resistivímetro Syscal PRO para CE, ambos da Iris Instruments............ 46

Figura 23 - Equipamentos utilizados na aquisição dos dados TDEM: a) Transmissor TDEM 57-MK2 e gerador; b) Receptor PROTEM e c) Bobina receptora 3D.

Figura 24 - Mapa de localização das aquisições de dados. Os quadrados azul e vermelho representam os loops transmissores e todos os círculos pretos dentro dos loops representam as sondagens TDEM. Os perfis de CE's são representados pelos pontos azuis $(\mathrm{E}-\mathrm{W})$, amarelos $(\mathrm{N}-\mathrm{S})$ e verdes $(\mathrm{N}-\mathrm{S})$ e as SEV's foram realizadas nas posições centrais dos dois loops e também na interface representada pela seta branca. Os perfis de CTDEM numerados de 1 a 3 foram utilizados nas Inversões Conjuntas 2D de CE e CTDEM. 48

Figura 25 - Fotos da terceira campanha de aquisição de dados: a) Aquisição SEV e b) Aquisição TDEM... 49

Figura 26 - Curva de dados TDEM brutos no software IX1D.............................................. 50

Figura 27 - Curva de dados TDEM editados no software IX1D. ............................................. 50

Figura 28 - Malha de diferenças finitas utilizada nas Inversões de CE. ................................. 51

Figura 29 - Detalhe da malha de diferenças finitas da Figura 28. ......................................... 51

Figura 30 - Modelo geoelétrico inicial homogêneo com $100 \Omega \cdot \mathrm{m}$, utilizado nas as Inversões de CE.

Figura 31 - Malha de diferenças finitas utilizada nas Inversões de CTDEM e nas Inversões Conjuntas 2D CE/CTDEM. 52

Figura 32 - Detalhe da malha de diferenças finitas da Figura 31. 53

Figura 33 - Modelo geoelétrico inicial homogêneo com $30 \Omega \cdot \mathrm{m}$, utilizado nas as Inversões de CTDEM e nas Inversões Conjuntas 2D CE/CTDEM. 53

Figura 34 - Resultado da Inversão do CE com arranjo polo-dipolo e espaçamento de eletrodos de $10 \mathrm{~m}$ na direção do perfil $1(\mathrm{E}-\mathrm{W})$. 54 
Figura 35 - Resultado da Inversão do CE com arranjo polo-dipolo e espaçamento de eletrodos de $20 \mathrm{~m}$ na direção do perfil 1 (E-W).

Figura 36 - Resultado da Inversão do CTDEM na direção do perfil 1 (E-W). 56

Figura 37 - Resultado da Inversão Conjunta 2D CE/CTDEM para o perfil 1 (E-W) com a escala de cores entre $10 \Omega \cdot \mathrm{m}$ e $100 \Omega \cdot \mathrm{m}$.

Figura 38 - Resultado da Inversão Conjunta 2D CE/CTDEM para o perfil 1 (E-W) com a escala de cores entre $5 \Omega \cdot \mathrm{m}$ e $45 \Omega \cdot \mathrm{m}$.

Figura 39 - Resultado da Inversão do CE com arranjo polo-dipolo e espaçamento de eletrodos

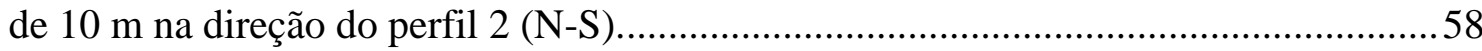

Figura 40 - Resultado da Inversão do CTDEM na direção do perfil 2 (N-S). 59

Figura 41 - Resultado da Inversão Conjunta 2D CE/CTDEM para o perfil $2(\mathrm{~N}-\mathrm{S})$ com a escala de cores entre $10 \Omega \cdot \mathrm{m}$ e $100 \Omega \cdot \mathrm{m}$.

Figura 42 - Resultado da Inversão Conjunta 2D CE/CTDEM para o perfil $2(\mathrm{~N}-\mathrm{S})$ com a escala de cores entre $5 \Omega \cdot \mathrm{m}$ e $45 \Omega \cdot \mathrm{m}$.

Figura 43 - Resultado da Inversão do CE com arranjo dipolo-dipolo e espaçamento de eletrodos de $20 \mathrm{~m}$ na direção do perfil $3(\mathrm{~N}-\mathrm{S})$.

Figura 44 - Resultado da Inversão do CTDEM na direção do perfil 3 (N-S). 62

Figura 45 - Resultado da Inversão Conjunta 2D CE/CTDEM para o perfil $3(\mathrm{~N}-\mathrm{S})$ com a escala de cores entre $10 \Omega \cdot \mathrm{m}$ e $100 \Omega \cdot \mathrm{m}$.

Figura 46 - Resultado da Inversão Conjunta 2D CE/CTDEM para o perfil $3(\mathrm{~N}-\mathrm{S})$ com a escala de cores entre $5 \Omega \cdot \mathrm{m}$ e $45 \Omega \cdot \mathrm{m}$

Figura 47 - Interpretação geológica do modelo geoelétrico obtido a partir da Inversão Conjunta para o perfil $1(\mathrm{E}-\mathrm{W})$.

Figura 48 - Interpretação geológica do modelo geoelétrico obtido a partir da Inversão Conjunta para o perfil $2(\mathrm{~N}-\mathrm{S})$.

Figura 49 - Interpretação geológica do modelo geoelétrico obtido a partir da Inversão Conjunta para o perfil $3(\mathrm{~N}-\mathrm{S})$. 65

Figura 50 - Mapa de espessura de sedimentos (modificado de Cogné et al., 2013). 67 


\section{Lista de Tabelas}

Tabela 1 - Poços em Taubaté cadastrados no SIAGAS-CPRM............................................ 10

Tabela 2 - Poços com informações sobre o nível água e vazão (SIAGAS-CPRM). ............... 11

Tabela 3 - Descrição do solo e litologia do poço P01 (SIAGAS-CPRM)............................... 12

Tabela 4 - Descrição do solo e litologia do poço mais profundo P40 (SIAGAS-CPRM)....... 12

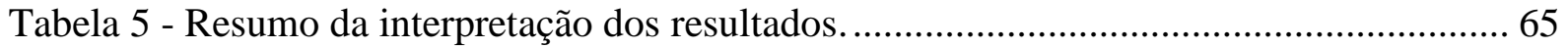




\section{RESUMO}

Nesta Pesquisa foi empregada a metodologia de Inversão Conjunta 2D de dados de caminhamento elétrico (CE) e caminhamento eletromagnético no domínio do tempo (CTDEM) num estudo hidrogeológico da Bacia de Taubaté. A área de estudo localiza-se na cidade de Taubaté/SP, onde o Aquífero Taubaté é uma importante fonte alternativa de água frente à recente crise hídrica. O Grupo Taubaté é o principal pacote sedimentar da bacia, sendo formado principalmente por folhelhos, que formam aquicludes, e arenitos, que formam o aquífero. Foram identificados 40 poços de exploração de água subterrânea na cidade, cadastrados no banco de dados do SIAGAS-CPRM, os quais forneceram informações importantes para a interpretação dos resultados. O objetivo principal da pesquisa é caracterizar a estratigrafia geoelétrica da subsuperfície para localizar o contato entre os sedimentos Quaternários e Terciários e mapear o aquífero. Os dados de CE permitem o reconhecimento das camadas mais rasas, da ordem de dezenas de metros, e os dados de CTDEM investigam as camadas mais profundas, numa ordem de centenas de metros, portanto, fornecem informações complementares. Os resultados das inversões conjuntas de CE/CTDEM permitiram mapear as camadas sedimentares mais rasas resistivas até aproximadamente $30 \mathrm{~m}$ de profundidade, uma camada sedimentar condutiva intermediária entre $30 \mathrm{~m}$ e $50 \mathrm{~m}$ de profundidade, e uma camada condutiva referente ao Grupo Taubaté entre $50 \mathrm{~m}$ e $300 \mathrm{~m}$ de profundidade. O topo do embasamento da bacia foi inferido em $\sim 300$ $\mathrm{m}$ de profundidade com base nas informações de poços e de geologia da bacia, por outro lado, também é possível que essa interface represente uma variação faciológica do Grupo Taubaté, portanto, mais estudos são necessários.

Palavras-chave: Caminhamento Elétrico (CE), Caminhamento Eletromagnético no Domínio do Tempo (CTDEM), Inversão Conjunta 2D CE/CTDEM, Bacia de Taubaté, Aquífero Taubaté. 


\begin{abstract}
In this research, a 2D Joint Inversion of electrical resistivity tomography (ERT) and time domain electromagnetic (TDEM) data was applied to a hydrogeological study of the Taubaté basin. The study area is in Taubaté city, São Paulo State, Brazil, where the Taubaté aquifer is an important alternative water source due to the recent water crisis. The Taubate Group is the main sedimentary package of the basin, which is formed mainly by shales, that form aquicludes, and sandstones that form the aquifer. 40 groundwater exploration wells were identified in the city, registered in SIAGAS-CPRM database, which provided important information for the results interpretation. The main objective of this research is to characterize the geoelectrical stratigraphy of the subsurface to locate the contact between the Quaternary and Tertiary sediments and to map the aquifer. The ERT is used for shallow investigations (tens of meters) and the TDEM can reach a great investigation depth (hundreds of meters), therefore, they provide complementary information. The 2D ERT/TDEM joint inversion results permitted to map the shallower resistive layers down to $30 \mathrm{~m}$ depth, an intermediate conductive layer between $30 \mathrm{~m}$ and $50 \mathrm{~m}$ depth, and the Taubate Group between $50 \mathrm{~m}$ and $300 \mathrm{~m}$ depth, which is very conductive. The top of the basement was inferred at $\sim 300 \mathrm{~m}$ depth based on the well information and the basin geology, on the other hand, it is also possible that this interface represents a faciological variation of the Taubaté Group, therefore, more studies are needed.
\end{abstract}

Keywords: Electrical Resistivity Tomography (ERT), 2D TDEM, 2D Joint Inversion ERT/TDEM, Taubaté Basin, Taubaté Aquifer. 


\section{INTRODUÇÃO E OBJETIVOS}

Desde 2014 o Brasil vem enfrentando uma crise hídrica, que é considerada a pior de sua história, devido principalmente à diminuição do nível das chuvas e o aumento do consumo de água, sendo a região Sudeste a mais afetada, pois é a região mais populosa e desenvolvida do país. Neste contexto, as águas subterrâneas são uma fonte alternativa extremamente importante. Dessa forma, os estudos hidrogeológicos são fundamentais e a geofísica é uma ferramenta que permite a investigação da subsuperfície de forma nãoinvasiva.

A área de estudo situa-se no município de Taubaté, estado de São Paulo, e encontra-se sobre a parte central da Bacia Sedimentar de Taubaté. As principais fontes de abastecimento público de água do município são os rios Paraíba do Sul e Una, sendo que a cidade vem sofrendo com os efeitos da crise hídrica. Por isso, o Aquífero Taubaté é uma fonte de água estratégica e são necessários estudos que permitam um aproveitamento sustentável deste recurso hídrico subterrâneo.

A Bacia de Taubaté apresenta feição tectônica de idade cenozoica e sua arquitetura rifte é caracterizada por semi-grabens (Riccomini et al., 2004). Dentro do banco de dados do Sistema de Informações de Águas Subterrâneas (SIAGAS) da Companhia de Pesquisa de Recursos Minerais (CPRM) foram identificados 40 poços em Taubaté, os quais fornecem informações de dados litológicos e de nível da água. O aquífero é do tipo multicamadas, com alternância de camadas arenosas ou aquíferas e argilosas ou confinantes. A principal área de descarga do aquífero é o Rio Paraíba do Sul. A parte central da bacia apresenta uma espessura saturada entre 200 e 300 metros e encontra-se dentro de um compartimento preenchido predominantemente por argilitos e folhelhos, pouco permeável e com características de aquiclude, apresentando vazões inferiores a $10 \mathrm{~m}^{3} / \mathrm{h}$, sendo, portanto, desfavorável para exploração de água subterrânea (DAEE et al., 1977).

Nesta pesquisa foram utilizados dois métodos geofísicos, o Método da Eletrorresistividade (ER) e o Método Eletromagnético no Domínio do Tempo (TDEM), sendo que ambos investigam a distribuição da resistividade elétrica em subsuperfície. Como as propriedades físicas variam muito para um mesmo mineral ou rocha, as ambiguidades estão sempre presentes no processo de interpretação dos resultados e, dessa forma, a utilização de mais de um método geofísico permite diminuir as ambiguidades e aumentar a confiabilidade da interpretação. 
No Método ER, a resistividade elétrica é obtida através da injeção de uma corrente elétrica no subsolo por meio de dois eletrodos e a medição da diferença de potencial por meio de outros pares de eletrodos. Já no Método Eletromagnético no Domínio do Tempo (TDEM), a distribuição da resistividade elétrica em subsuperfície é obtida através da indução eletromagnética nos materiais condutores e a medida da variação desse campo eletromagnético induzido em função do tempo.

Ambos os métodos possuem vantagens e limitações em termos de resolução e profundidade de investigação, assim como todos os métodos geofísicos. O método ER é normalmente utilizado em investigações rasas, da ordem de dezenas de metros, devido à sua limitação logística, pois requer longas aberturas de eletrodos para obter maiores profundidades. Por outro lado, o método TDEM possui baixa resolução para as camadas mais rasas e normalmente é utilizado em investigações profundas, da ordem de centenas de metros, sendo capaz de definir camadas condutivas. Portanto, os métodos ER e TDEM podem ser utilizados de forma complementar.

Os dois métodos são amplamente aplicados em estudos hidrogeofísicos, devido à rápida aquisição de dados, custo relativamente baixo e confiabilidade. Eles têm o potencial de identificar aquíferos ${ }^{1}$, aquicludes ${ }^{2}$ e aquitardes ${ }^{3}$, que normalmente são mais condutores do que as camadas circundantes. Além disso, eles também são sensíveis a propriedades geológicas, como, por exemplo, o conteúdo de argila, porosidade e permeabilidade.

Um procedimento satisfatório para a integrar dados ER e TDEM é através da inversão conjunta. Neste caso, a inversão dos dados ER e TDEM é feita simultaneamente, ou seja, o mesmo modelo geoelétrico deve satisfazer ao mesmo tempo todo o conjunto de dados. Os trabalhos pioneiros relacionados à inversão conjunta são atribuídos a Vozoff \& Jupp (1975), que utilizaram dados magnetotelúricos (MT) e sondagens elétricas verticais (SEV). Nesta mesma linha de pesquisa, alguns trabalhos mostraram as vantagens da inversão conjunta de dados de ER e TDEM (Raiche et al., 1985; Meju, 1996; Yang \& Tong, 1999) e também aplicações da inversão conjunta em estudos hidrogeológicos (Goldman et al., 1994; Albouy et al., 2000). No Brasil, as pesquisas pioneiras na área de inversão conjunta 1D de dados SEV e TDEM para estudos de água subterrânea são atribuídas a Bortolozo (2011). Outros

\footnotetext{
1 Aquífero é um meio rochoso que tem capacidade para armazenar e transmitir água, ou seja, que possui porosidade e permeabilidade. Exemplo: arenito.

${ }_{2}^{2}$ Aquiclude é um meio rochoso que embora possa armazenar água, não a transmite, isto é, possui porosidade, mas é impermeável. Exemplo: argilito.

3 Aquitarde é um meio rochoso que armazena água, porém a transmite muito lentamente, isto é, possui porosidade e permeabilidade baixas. Exemplo: arenito com matriz argilosa.
} 
pesquisadores também usaram a inversão de dados para estudos de água subterrânea, como, por exemplo, Porsani et al. (2012a, 2012b), Leite (2013), Bortolozo et al. (2014, 2015), Campaña (2015), Couto Jr. (2015), Campaña et al. (2017) e Leite et al. (2018). Os estudos de inversão conjunta 2D de dados de Caminhamento Elétrico (CE) e Caminhamento TDEM (CTDEM) são atribuídos a Bortolozo (2016), sendo, portanto, a área de inversão conjunta 1D/2D ainda pouco explorada.

Nesta pesquisa é empregada a metodologia de Inversão Individual e Conjunta 2D de dados de CE e CTDEM (Bortolozo, 2016), que consiste em um conjunto de rotinas em MATLAB para modelagem e inversão.

Foram realizadas três campanhas de aquisições de dados nos meses de abril, junho e setembro de 2016, onde foram adquiridos dados ER e TDEM em uma e duas dimensões, no entanto, nesta pesquisa foram utilizados apenas os dados 2D.

O objetivo principal desta pesquisa é caracterizar a estratigrafia geoelétrica da área de estudo, visando localizar o contato entre os sedimentos Quaternários e Terciários e mapear o Aquífero Taubaté. Os objetivos secundários são: contribuir com o aprimoramento da metodologia de Inversão Individual e Conjunta 2D de dados de CE e CTDEM e na criação de um tutorial para que as rotinas de modelagem e inversão possam ser utilizadas em trabalhos futuros; contribuir com o avanço na interpretação dos métodos geoelétricos num contexto geológico da Bacia de Taubaté; e corroborar com as pesquisas hidrogeofísicas no Estado de São Paulo. 


\section{2 ÁREA DE ESTUDO}

A área de estudo situa-se numa área rural a cerca de $6 \mathrm{~km}$ a nordeste do centro de Taubaté/SP, que se encontra sobre a porção central da Bacia de Taubaté. Taubaté (Figura 1) é uma cidade média, com aproximadamente 308.000 habitantes (IBGE, 2017), localizada na região do Vale do Paraíba a cerca de $130 \mathrm{~km}$ da capital do estado, São Paulo. As principais fontes de abastecimento público de água do município são os rios Paraíba do Sul e Una.

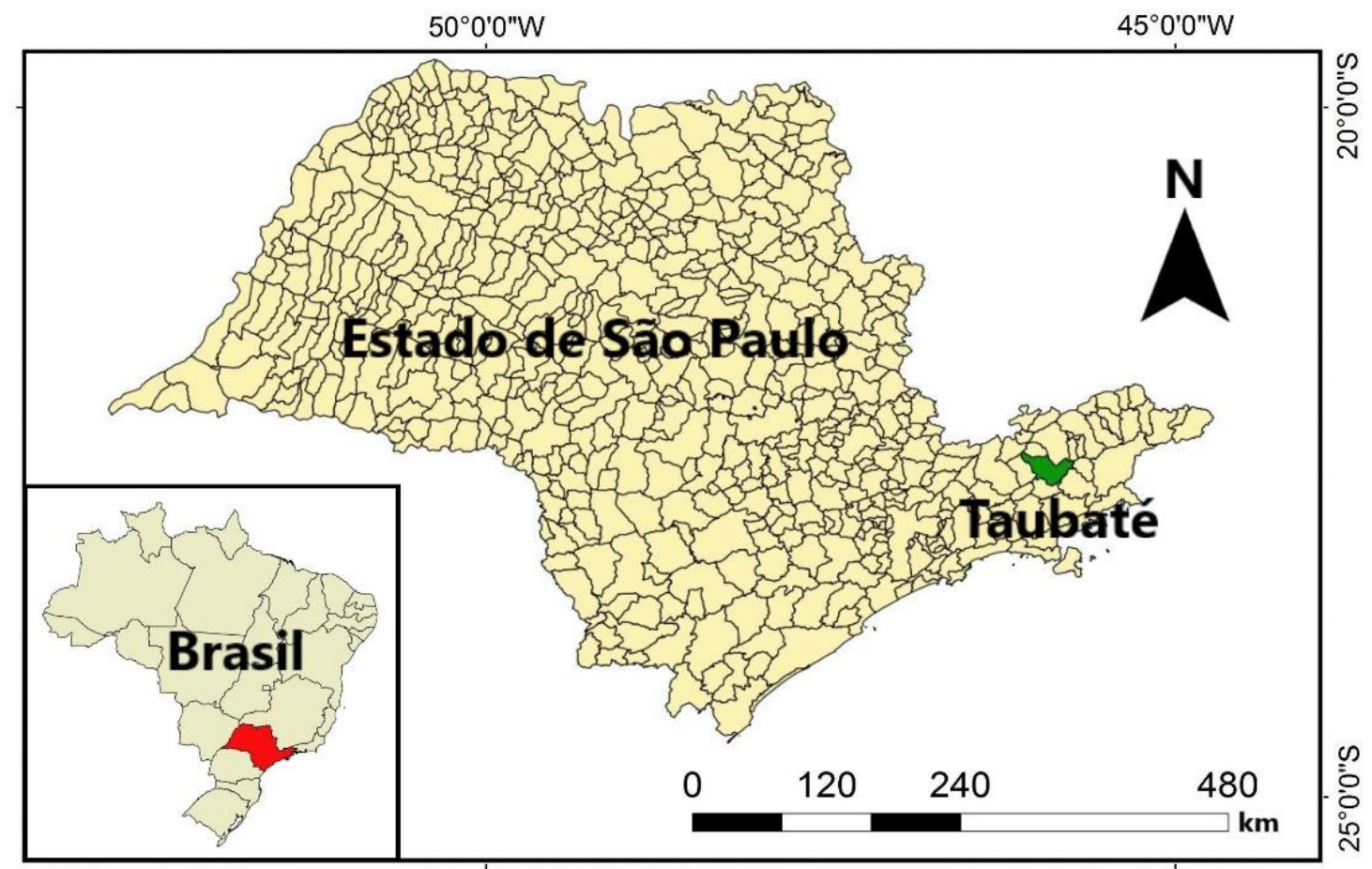

Figura 1 - Mapa de localização da cidade de Taubaté/SP.

\subsection{Aspectos Geológicos da Bacia de Taubaté}

A Bacia de Taubaté localiza-se na parte central do Rifte Continental do Sudeste do Brasil (RCSB) (Riccomini, 1989). O RCSB é formado por um conjunto de bacias sedimentares e abrange mais de $900 \mathrm{~km}$ entre as cidades de Curitiba (PR) e Barra de São João (RJ), formando uma faixa estreita e alongada que acompanha a linha costeira com direção ENE. A Bacia de Taubaté é a maior destas bacias, com $170 \mathrm{~km}$ de comprimento e $20 \mathrm{~km}$ de largura (Riccomini et al., 2004), ocorrendo na região leste do estado de São Paulo. Localizada no Planalto Atlântico, a bacia está posicionada entre as Serras do Mar e Mantiqueira e a espessura estimada dos sedimentos que a compõem pode atingir até 850 metros (Vidal, 2004). A Figura 2 apresenta um mapa geológico da bacia com a localização da área de estudo. 


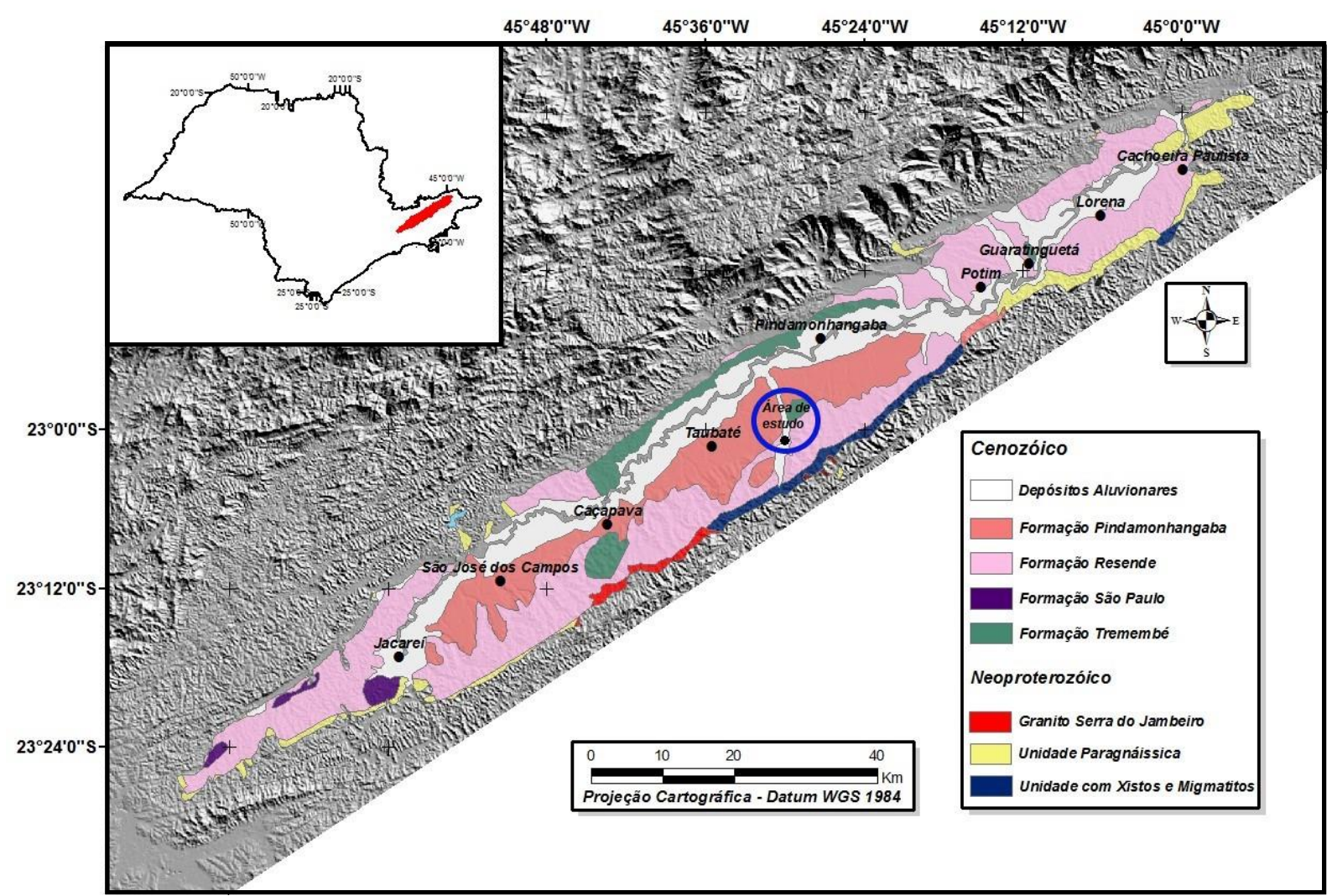

Figura 2 - Localização da Bacia de Taubaté e da área de estudo (modificado de Souza Filho et al., 2013).

A bacia está relacionada à tectônica distensiva de idade terciária e é caracterizada por uma série de semi-grabens preenchidos com sedimentos Terciários e Quartenários. A Figura 3 apresenta um quadro litoestratigráfico e a evolução tectono-sedimentar do segmento central do RCSB.

A bacia desenvolveu-se sobre rochas metamórficas e rochas ígneas de idade proterozóica, referentes ao Cinturão Ribeira (Almeida et al.,1973) e rochas alcalinas de idade Neocretácea associados a diques e sills (Riccomini, 1989). O preenchimento sedimentar é basicamente continental e pode ser dividido em duas fases: a primeira, sintectônica ao rifte, com a deposição dos sedimentos do Grupo Taubaté; e a segunda, posterior à tectônica diastrófica, com a deposição das Formações Itaquaquecetuba e Pindamonhangaba e depósitos aluviais e coluviais (Riccomini, 1989).

O principal pacote sedimentar da bacia é representado pelo Grupo Taubaté, que é subdividido pelas formações Resende, Tremembé e São Paulo. A Formação Resende, que é o pacote mais abundante do Grupo Taubaté, representa um sistema de leques aluviais associados à planície aluvial de rios entrelaçados, sendo formada por orto e paraconglomerados nas áreas proximais e lamitos nas porções medianas e distais dos leques aluviais, além de arenitos fluviais em rios entrelaçados. A Formação Tremembé, localizada na 
porção central da bacia, se caracteriza por um sistema lacustre eocênico-oligocênico formado por argilitos verdes maciços, ritmitos de folhelhos e margas, dolomitos e arenitos. A Formação São Paulo, localizada no topo do Grupo Taubaté, é caracterizada por um sistema fluvial meandrante oligocênico, contendo como principais litofácies arenitos grossos conglomeráticos e estratificados, siltitos e argilitos laminados, depositados em meandros abandonados, e arenitos médios a grossos, gradando para sedimentos mais finos, rítmicos e laminados de planície de inundação (Riccomini et al., 2004).

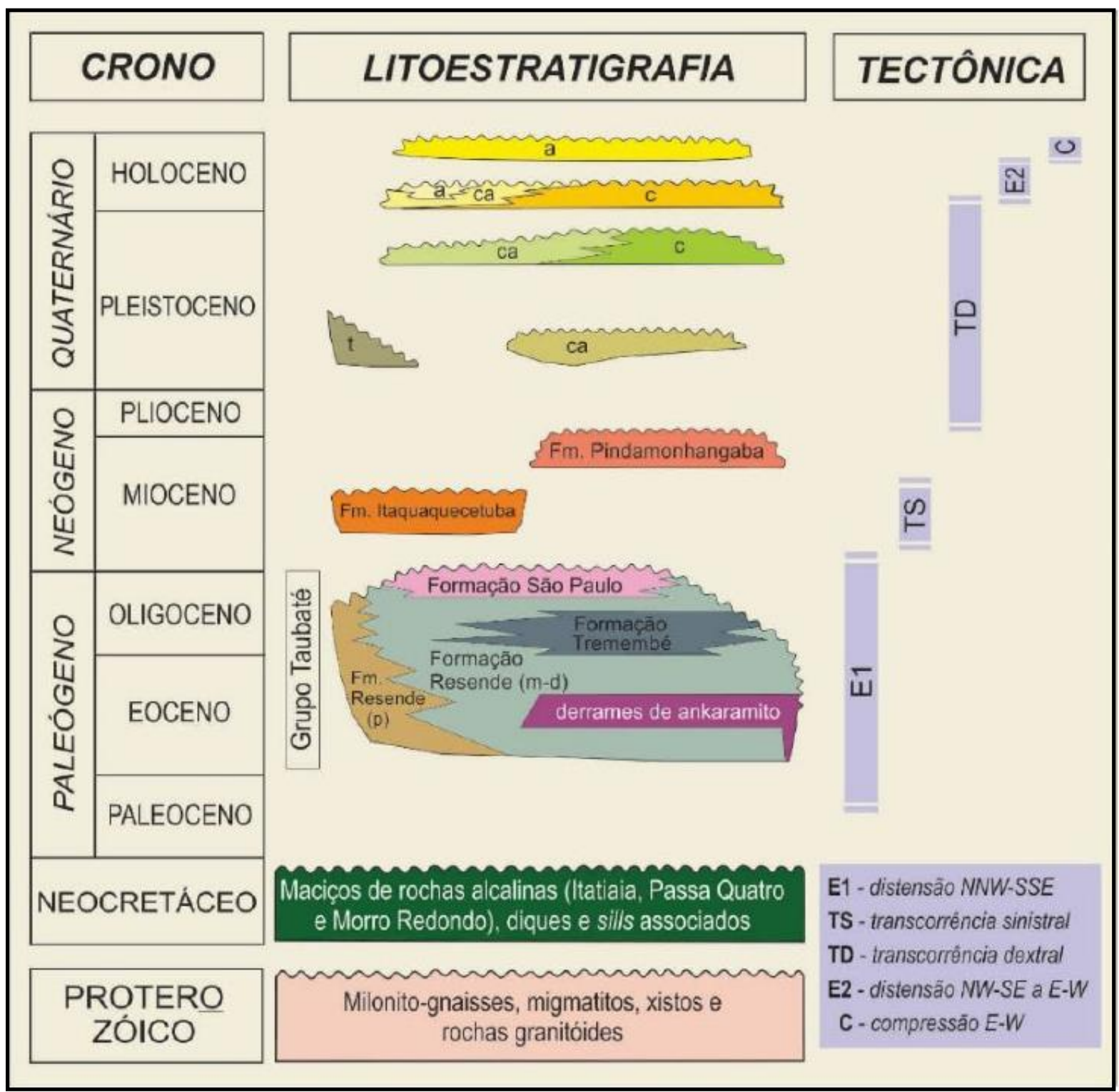

Figura 3 - Quadro litoestratigráfico e evolução tectono-sedimentar do segmento central do RCSB - Letras: p - leques aluviais proximais; m-d - leques aluviais medianos a distais associados a planície aluvial de rios entrelaçados; $\mathbf{t}$ - depósitos de tálus; $\mathbf{c}$ - depósitos coluviais; ca - depósitos colúvio-aluviais; a - depósitos aluviais (modificado de Riccomini et al., 2004). 
No Neógeno, o segmento central do RCSB foi preenchido por sedimentos referentes às formações Itaquaquecetuba e Pindamonhangaba. A Formação Itaquaquecetuba, encontrada apenas na Bacia de São Paulo, caracteriza-se essencialmente por arenitos grossos arcoseanos, pobremente a medianamente selecionados, eventualmente contendo níveis argilo-siltosos, ricos em matéria orgânica e apresentando níveis de conglomerado com seixos de quartzo e quartzito bem arredondados (Riccomini et al., 2004). Estratigraficamente acima da Formação Itaquaquecetuba, encontra-se a Formação Pindamonhangaba, formada por arenitos, conglomerados, argilitos e siltitos, referente a um sistema fluvial meandrante desenvolvido no centro e sudeste da bacia e aflorante em faixa alongada ao sul do rio Paraíba do Sul (Riccomini et al., 2004).

Por último, no período Quaternário, ocorrem sedimentos aluviais, coluviais, colúvioaluviais e de depósitos de tálus, como pode ser observado na Figura 3.

\subsection{Aquífero Taubaté}

O Aquífero Taubaté ocorre em duas áreas principais da bacia, uma situada na porção sudoeste e outra a nordeste. Entre estas duas regiões existe um compartimento preenchido predominantemente por argilitos e folhelhos, pouco permeável e com características de aquiclude (DAEE, 1977), onde se localiza a cidade de Taubaté. Como resultado de seus ambientes deposicionais, o aquífero é do tipo multicamadas com alternância de camadas arenosas ou aquíferas, associadas a fácies fluviais, e camadas argilosas ou confinantes, associadas a fácies lacustre ou de planície de inundação (DAEE, 1977).

$\mathrm{Na}$ área central da bacia, a base do aquífero encontra-se em cotas topográficas entre 100 e 250 m (DAEE, 1977). As cotas da base aumentam na direção dos limites da bacia, atingindo elevações de $550 \mathrm{~m}$ próximo às áreas de afloramento de rochas do embasamento. $\mathrm{Na}$ parte central da bacia ocorrem as maiores espessuras de sedimentos, diminuindo em direção às margens e também à noroeste. A Figura 4 apresenta um mapa da espessura saturada do aquífero indicando a localização da área de estudo, onde é possível observar que a espessura saturada é maior do que $250 \mathrm{~m}$. 


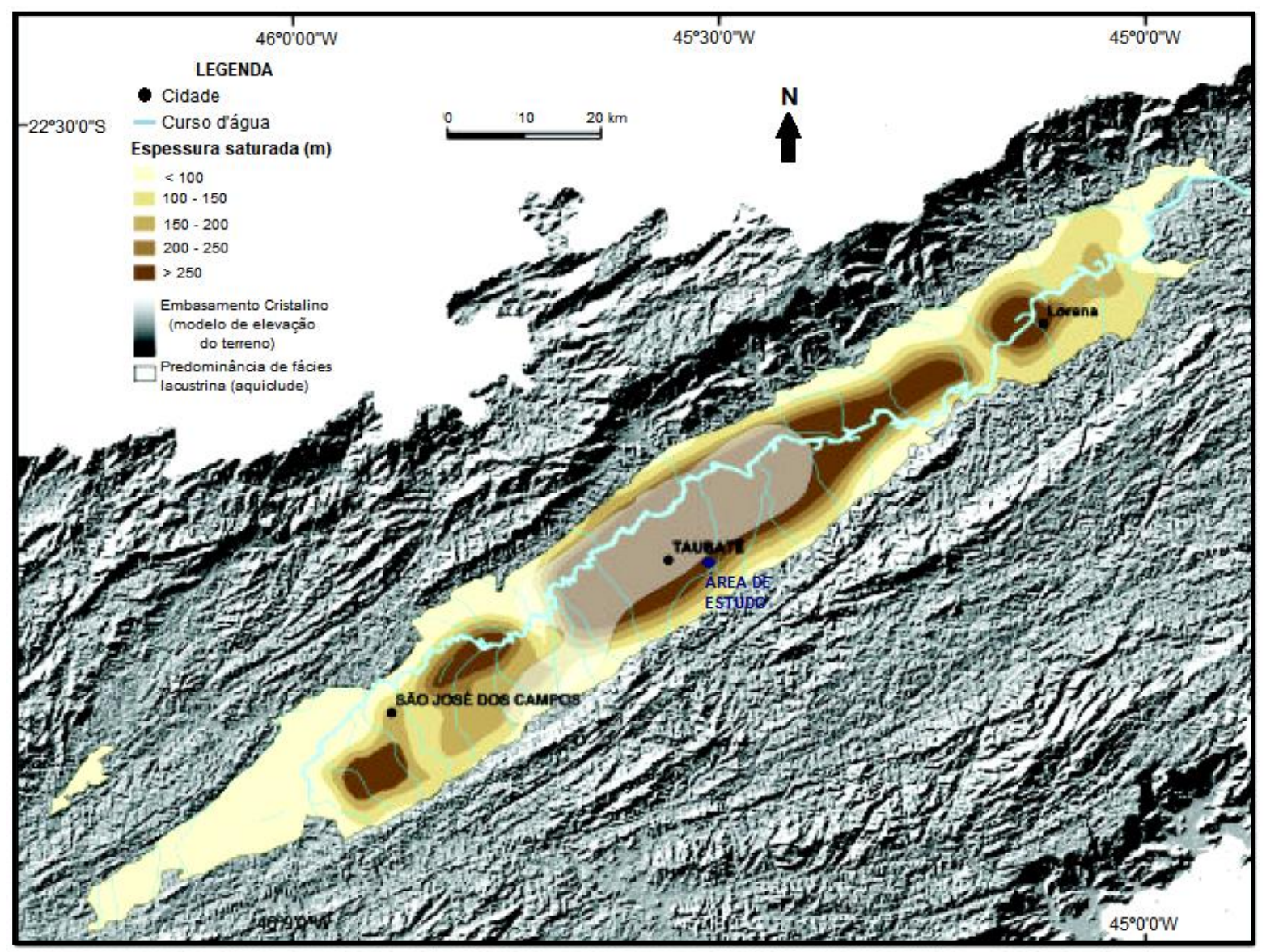

Figura 4 - Espessura saturada do Aquífero Taubaté (modificado de DAEE et al., 2005).

O aquífero ocorre, principalmente, de forma livre ou pouco confinada, observando-se poucas áreas sob pressão (DAEE, 1977). As cotas potenciométricas variam de $600 \mathrm{~m}$, nos limites da bacia, a $550 \mathrm{~m}$ nas proximidades do rio Paraíba do Sul, principal área de descarga natural das águas do aquífero. De acordo com o relatório do DAEE et al. (2005), as regiões do eixo Jacareí - São José dos Campos, Caçapava, Lorena e Guaratinguetá são consideradas as mais promissoras para exploração de água subterrânea, com vazões entre 80 e $120 \mathrm{~m}^{3} / \mathrm{h}$, enquanto a região central da bacia, entre os municípios de Taubaté e Pindamonhangaba, apresenta características menos favoráveis, com vazões inferiores a $10 \mathrm{~m}^{3} / \mathrm{h}$. Vazões entre 40 e $80 \mathrm{~m}^{3} / \mathrm{h}$, observadas no sudoeste da bacia, nas proximidades de Roseira, resultam, principalmente, do aumento da espessura saturada do aquífero nesta região. 


\subsection{Poços}

Foram identificados 40 poços de exploração de água subterrânea na cidade de Taubaté cadastrados no banco de dados do Sistema de Informações de Águas Subterrâneas (SIAGAS) da Companhia de Pesquisa de Recursos Minerais (CPRM). A Figura 5 apresenta um mapa de localização dos poços e a Tabela 1 apresenta as coordenadas, profundidades e uso da água. Todos os poços são tubulares e a água é utilizada principalmente para abastecimento doméstico ou industrial.

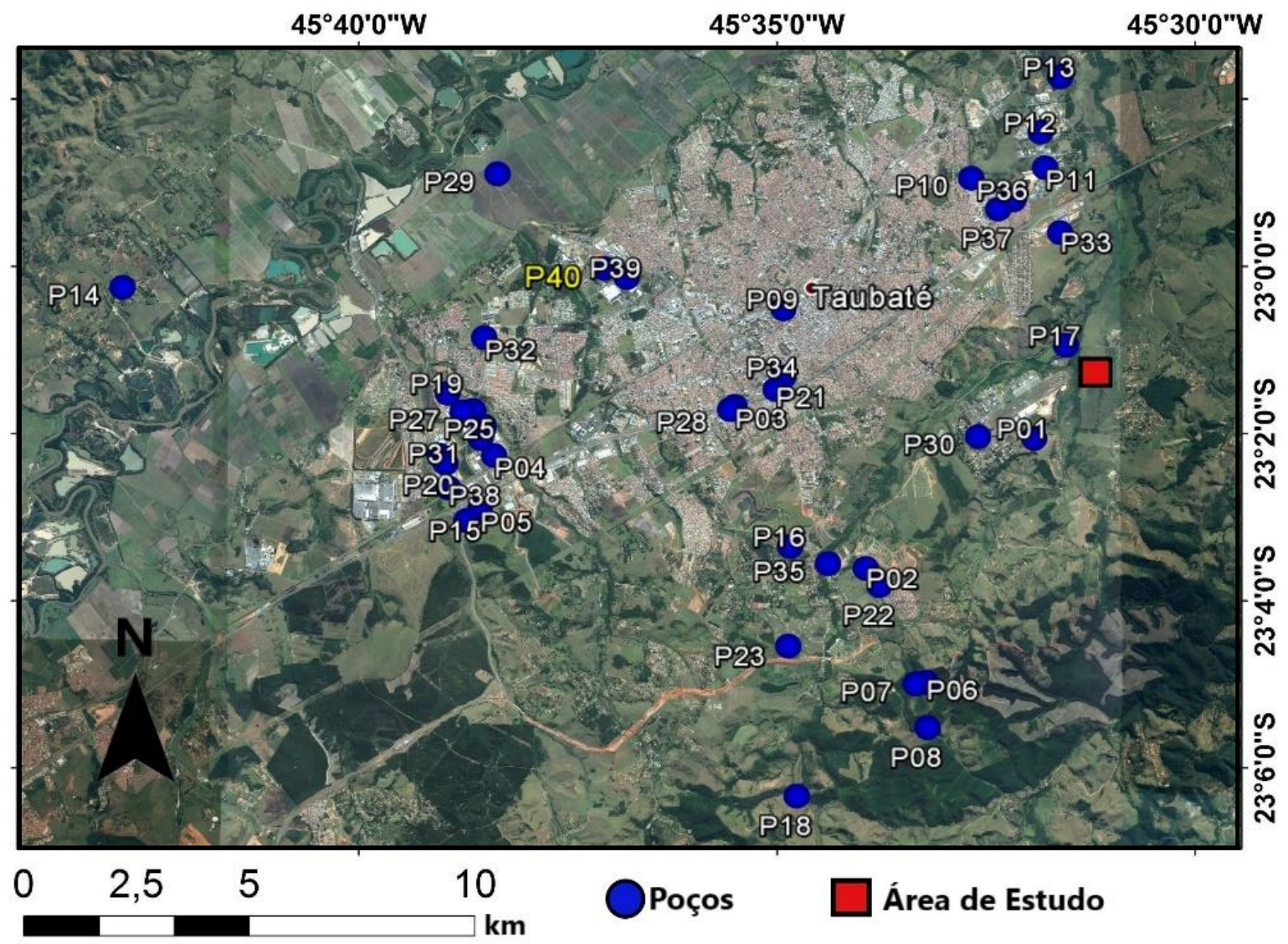

Figura 5 - Localização dos poços em Taubaté cadastrados no SIAGAS-CPRM e da área de estudo, com destaque para o poço mais profundo $(\mathbf{P 4 0})$. 
Tabela 1 - Poços em Taubaté cadastrados no SIAGAS-CPRM.

\begin{tabular}{|c|c|c|c|c|}
\hline Poço & Latitude $\left(^{\circ}\right)$ & Longitude $\left(^{\circ}\right)$ & Profundidade (m) & Uso da água \\
\hline $\mathrm{P} 01$ & -23.0472 & -45.5153 & 124 & Abastecimento doméstico \\
\hline $\mathrm{P} 02$ & -23.0706 & -45.5461 & 186 & - \\
\hline P03 & -23.0417 & -45.5703 & 122,5 & Outros (lazer, etc.) \\
\hline P04 & -23.0506 & -45.6144 & 120 & Abastecimento industrial \\
\hline P05 & -23.0603 & -45.6169 & 270 & Abastecimento doméstico \\
\hline P06 & -23.0908 & -45.5350 & 80 & Abastecimento industrial \\
\hline P07 & -23.0911 & -45.5369 & 100 & Abastecimento industrial \\
\hline P08 & -23.0989 & -45.5347 & 156 & Outros (lazer, etc.) \\
\hline P09 & -23.0242 & -45.5614 & 95 & Abastecimento industrial \\
\hline $\mathrm{P} 10$ & -23.0006 & -45.5269 & 60 & Abastecimento industrial \\
\hline P11 & -22.9986 & -45.5133 & 120 & Abastecimento industrial \\
\hline $\mathrm{P} 12$ & -22.9922 & -45.5142 & 340 & Abastecimento industrial \\
\hline $\mathrm{P} 13$ & -22.9822 & -45.5106 & 352 & Abastecimento industrial \\
\hline P14 & -23.0206 & -45.6831 & 200 & Abastecimento doméstico \\
\hline $\mathrm{P} 15$ & -23.0622 & -45.6194 & 314 & Abastecimento doméstico \\
\hline P16 & -23.0667 & -45.5600 & 134 & Abastecimento doméstico \\
\hline P17 & -23.0306 & -45.5094 & 115 & Irrigação \\
\hline $\mathrm{P} 18$ & -23.1111 & -45.5586 & 249 & Outros (lazer, etc.) \\
\hline P19 & -23.0394 & -45.6231 & 70 & Abastecimento industrial \\
\hline $\mathrm{P} 20$ & -23.0506 & -45.6244 & 70 & Abastecimento industrial \\
\hline $\mathrm{P} 21$ & -23.0386 & -45.5628 & 38 & Abastecimento industrial \\
\hline $\mathrm{P} 22$ & -23.0736 & -45.5436 & 122 & Abastecimento urbano \\
\hline $\mathrm{P} 23$ & -23.0844 & -45.5603 & 240 & Abastecimento industrial \\
\hline $\mathrm{P} 24$ & -23.0425 & -45.6183 & 370 & Abastecimento industrial \\
\hline $\mathrm{P} 25$ & -23.0475 & -45.6169 & 350 & Abastecimento industrial \\
\hline $\mathrm{P} 26$ & -23.0453 & -45.6164 & 200 & Outros (lazer, etc.) \\
\hline P27 & -23.0428 & -45.6203 & 80 & Abastecimento industrial \\
\hline P28 & -23.0422 & -45.5711 & 200 & Abastecimento doméstico \\
\hline P29 & -23.0000 & -45.6142 & 393 & Abastecimento industrial \\
\hline $\mathrm{P} 30$ & -23.0469 & -45.5256 & 172 & Outros (lazer, etc.) \\
\hline P31 & -23.0519 & -45.6233 & 100 & Abastecimento industrial \\
\hline $\mathrm{P} 32$ & -23.0294 & -45.6164 & 35 & Abastecimento industrial \\
\hline P33 & -23.0103 & -45.5106 & 220 & Abastecimento industrial \\
\hline P34 & -23.0364 & -45.5614 & 155 & Outros (lazer, etc.) \\
\hline P35 & -23.0697 & -45.5531 & 112,5 & - \\
\hline P36 & -23.0044 & -45.5192 & 132 & Abastecimento industrial \\
\hline P37 & -23.0061 & -45.5219 & 123 & Abastecimento industrial \\
\hline P38 & -23.0564 & -45.6228 & 100 & Abastecimento industrial \\
\hline P39 & -23.0186 & -45.5903 & 442,3 & Abastecimento industrial \\
\hline $\mathrm{P} 40$ & -23.0169 & -45.5944 & 650 & Abastecimento industrial \\
\hline
\end{tabular}


A Tabela 2 apresenta os poços que possuem informações sobre o nível de água e a vazão, onde o nível estático é medido antes do bombeamento e, o nível dinâmico, após o bombeamento. Com exceção do poço P02, observa-se que a vazão em geral é baixa, sendo coerente com a informação disponível no relatório do DAEE et al. (2005).

Tabela 2 - Poços com informações sobre o nível água e vazão (SIAGAS-CPRM).

\begin{tabular}{ccccc}
\hline Poço & Cota $(\mathbf{m})$ & Nível estático $(\mathbf{m})$ & Nível dinâmico $(\mathbf{m})$ & Vazão $\left(\mathbf{m}^{\mathbf{3}} \mathbf{h}\right)$ \\
\hline P01 & - & 42 & 56 & 6 \\
P02 & 590 & 17,6 & 75,47 & 34,5 \\
P03 & - & 20,05 & 95,6 & 10 \\
P04 & 586 & 18 & 110 & 1,5 \\
P05 & 600 & 34,6 & 195 & 10,35 \\
P06 & 632 & 2 & 56 & 3 \\
P07 & 638 & 0 & 70 & 3 \\
P08 & 602 & 0,2 & 33,5 & 5 \\
P09 & 560 & 7,2 & 65,78 & 1,5 \\
\hline
\end{tabular}

Seis poços atingiram o embasamento da bacia, que é formado principalmente por gnaisses. Os poços P06, P07, P08 e P18 encontram-se próximos a borda da bacia e atingiram o embasamento em menos de 20 m de profundidade. Já os poços P39 e P40 encontram-se no centro da bacia e atingiram o embasamento em $442 \mathrm{~m}$ e $510 \mathrm{~m}$ de profundidade, respectivamente.

O poço mais próximo da área de estudo, P17, encontra-se cerca de $750 \mathrm{~m}$ de distância e possui $115 \mathrm{~m}$ de profundidade, no entanto, a sua descrição geológica inicia-se em $80 \mathrm{~m}$ de profundidade, por isso, contribui pouco com a interpretação dos resultados.

O segundo poço mais próximo é o P01, a cerca de $1,8 \mathrm{~km}$ de distância da área de estudo. Este poço possui $124 \mathrm{~m}$ de profundidade e encontra-se em uma altitude de $624 \mathrm{~m}$. Como a área de estudo encontra-se em uma altitude média de $565 \mathrm{~m}$, portanto, o poço P01 encontra-se a cerca de $60 \mathrm{~m}$ acima da área de estudo. A Tabela 3 apresenta a descrição de solo e litologia do poço P01. 
Tabela 3 - Descrição do solo e litologia do poço P01 (SIAGAS-CPRM).

\begin{tabular}{cccc}
\hline De $(\mathbf{m})$ & Até $(\mathbf{m})$ & Solo / Litologia & Descrição \\
\hline 0 & 12 & Solo & Solo argilo-arenoso \\
12 & 14 & Arenito argiloso & Amarelo, muito fino \\
14 & 26 & Arenito & Amarelo, grosso, argiloso (5\%) \\
26 & 36 & Arenito argiloso & Amarelo, argiloso (50\%) \\
36 & 44 & Argilito & Verde \\
44 & 60 & Arenito argiloso & Amarelo, médio/grosso, argiloso (30\%) \\
60 & 66 & Arenito argiloso & Verde, grosso, argiloso (30\%) \\
66 & 74 & Argilito & Verde \\
74 & 104 & Arenito argiloso & Amarelo/verde, médio/grosso, argiloso (50\%) \\
104 & 124 & Argilito & Verde \\
\hline
\end{tabular}

A Tabela 4 apresenta a descrição do solo e da litologia do poço mais profundo, o P40, o qual encontra-se a cerca de 9,5 km de distância da área de estudo e em $568 \mathrm{~m}$ de altitude, ou seja, uma altitude muito próxima da área de estudo. O poço P40 é considerado o poço mais representativo e que mais contribuiu para a interpretação dos resultados. Na Tabela 4, as rochas entre 16 e $510 \mathrm{~m}$ de profundidade provavelmente representam o Grupo Taubaté.

Tabela 4 - Descrição do solo e litologia do poço mais profundo P40 (SIAGAS-CPRM).

\begin{tabular}{cccc}
\hline De $(\mathbf{m})$ & Até $(\mathbf{m})$ & Solo / Litologia & Descrição \\
\hline 0 & 4 & Solo arenoso & Solo arenoso avermelhado \\
4 & 11 & Argila & Material argiloso variegado \\
11 & 16 & Arenito médio & Arenito conglomerático, friável, mal selecionado \\
16 & 401 & Folhelho & Folhelho esverdeado \\
401 & 485 & Folhelho & Folhelho misturado com seixos gnáissicos (10 a 50\%) \\
485 & 493 & Arenito argiloso & Arenito médio/fino com argila cinza claro \\
493 & 510 & Folhelho & Folhelho misturado com seixos gnáissicos/quartizíticos \\
510 & 650 & Gnaisse & Gnaisse rico em biotita com veios de quartzo \\
\hline
\end{tabular}

Em geral, a partir das descrições de todos os poços, o Grupo Taubaté é formado principalmente por folhelhos ou argilitos intercalados por camadas de arenito, sendo coerente com as informações da geologia. Todas as informações dos poços são muito importantes para o processo de interpretação dos resultados. 


\section{MÉTODOS GEOFÍSICOS}

Nesta pesquisa foram utilizados dois métodos geofísicos: o método da eletrorresistividade (ER) e o método eletromagnético no domínio do tempo (TDEM). Ambos os métodos permitem investigações acerca da mesma propriedade física dos materiais em subsuperfície, a resistividade elétrica $(\rho)$ ou condutividade elétrica $(\sigma=1 / \rho)$.

No método ER a investigação é feita através da injeção de correntes elétricas e, no método TDEM, através da indução eletromagnética nos materiais condutores em subsuperfície. Os dois métodos utilizam fontes artificiais e a resposta é medida na superfície. Dessa forma, é possível relacionar a distribuição da resistividade elétrica com a geologia ou variações na composição litológica, como, por exemplo, presença de água, fraturas e/ou mineralogia.

A resistividade elétrica é uma propriedade intrínseca dos materiais geológicos e é definida como a medida da oposição à passagem de uma corrente elétrica através deste material, ou seja, quanto menor a resistividade, mais facilmente o material permite a passagem de uma corrente elétrica. Numericamente, a resistividade é definida como a resistência (em ohms) entre as faces opostas de um cubo unitário do material. Assim, para um cilindro condutor infinitesimal de resistência $d R$, comprimento $d L$ e área de seção transversal $d A$, a resistividade é dada por:

$$
\rho=\frac{d R d A}{d L}
$$

onde, no sistema internacional (SI), a unidade da resistividade é o $\Omega \cdot \mathrm{m}(\mathrm{Ohm} \cdot \mathrm{metro})$.

O valor da resistividade elétrica pode variar muito para um mesmo tipo de rocha. Alguns minerais são bons condutores de eletricidade, como é o caso dos metais nativos (ouro, prata, cobre, etc.), porém, a maioria dos minerais formadores de rochas é isolante. Rochas sedimentares conduzem eletricidade principalmente através dos fluidos condutivos (soluções eletrolíticas) presentes em seus poros. Portanto, a porosidade é o principal fator que controla a resistividade das rochas sedimentares e, geralmente, a resistividade aumenta com a diminuição da porosidade. As rochas cristalinas possuem porosidade intergranular insignificante, no entanto, podem conduzir eletricidade através de fluidos presentes em fraturas ou fissuras. Assim, além da porosidade e das fraturas ou fissuras, a resistividade também depende da quantidade e da natureza do fluido presente na rocha. Portanto, para 
relacionar as informações dos dados geofísicos com a geologia da área de estudo, é fundamental o conhecimento da geologia local em termos litoestratigráficos.

O interior da terra é heterogêneo, ou seja, é formado por camadas com diferentes valores de resistividade, por isso a resistividade obtida por ambos os métodos representa a média ponderada das resistividades de todas as camadas por onde o sinal passou, denominado de resistividade aparente. A resistividade de cada camada geoelétrica é determinada através do processamento ou inversão dos dados.

A seguir serão apresentados os fundamentos teóricos e as principais características dos métodos ER e TDEM.

\subsection{Método da Eletrorresistividade (ER)}

O método da eletrorresistividade consiste em injetar uma corrente elétrica no subsolo através de eletrodos metálicos cravados na superfície do terreno (eletrodos de corrente) e a medição do potencial resultante por pares de eletrodos (eletrodos de potencial). Dessa forma é possível obter a distribuição da resistividade elétrica da subsuperfície.

\subsubsection{Princípios Físicos do Método ER}

Pela lei de Ohm, a corrente elétrica (I) que passa por um elemento infinitesimal cilíndrico de um material homogêneo é dada por:

$$
I=-\frac{d V}{d R}
$$

onde $d V$ é a diferença de potencial entre os extremos do material e $d R$ é a sua resistência elétrica. O sinal negativo na equação (2) significa que a corrente elétrica se desloca do ponto de maior para menor potencial elétrico.

Isolando $d R$ na equação (1) e substituindo na equação (2), obtém-se:

$$
\frac{d V}{d L}=-\frac{\rho I}{d A}
$$

A Figura 6 representa um eletrodo pontual sobre uma superfície infinita, homogênea e 
isotrópica com resistividade $\rho$. O circuito é fechado para uma fonte de corrente colocado a uma grande distância do eletrodo. A corrente elétrica flui radialmente a partir do eletrodo e sua distribuição é uniforme sobre cascas hemisféricas centradas no eletrodo. Portanto, para uma casca hemisférica de raio $r$ e espessura $d r$, a partir da equação (3) obtém-se:

$$
\frac{d V}{d r}=-\frac{\rho I}{2 \pi r^{2}}
$$

onde $2 \pi r^{2}$ é a área superficial da casca hemisférica.

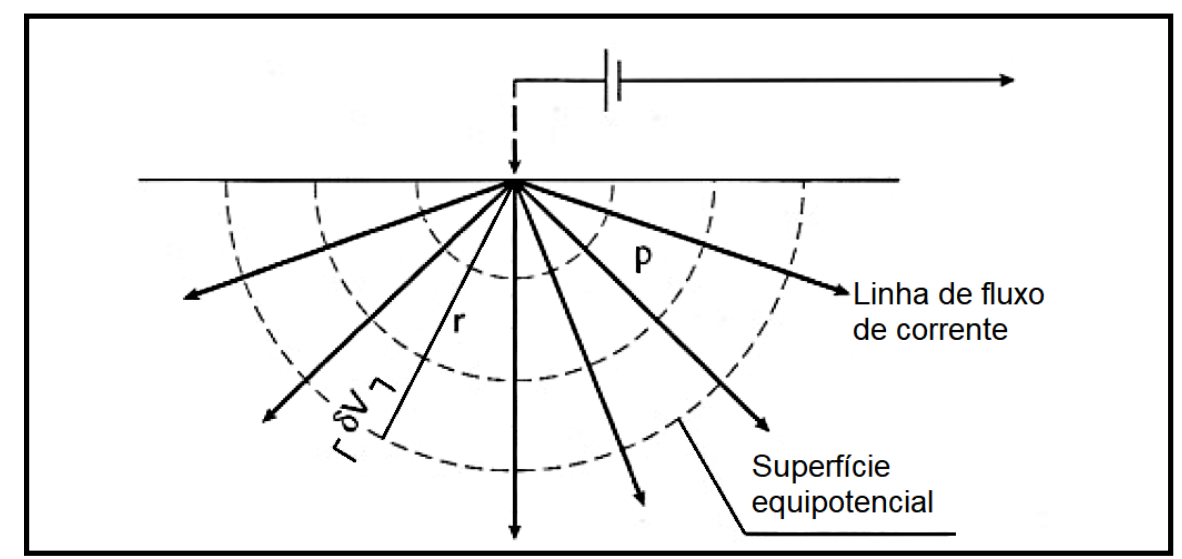

Figura 6 - Eletrodo pontual na superfície de um meio homogêneo e isotrópico (adaptado de Kearey et al., 2002).

Integrando a equação (4), obtém-se o potencial a uma distância $r$ da fonte:

$$
V(r)=\frac{\rho I}{2 \pi r}+C
$$

onde $C$ é uma constante arbitrária. Assumindo o potencial como sendo nulo no infinito, então $C=0$. Assim, a equação (5) fica na forma:

$$
V(r)=\frac{\rho I}{2 \pi r}
$$

A equação (6) é a mais importante para o método ER, pois fornece o potencial elétrico para qualquer ponto abaixo do eletrodo, ou seja, na subsuperfície.

$\mathrm{Na}$ prática são utilizados dois eletrodos de corrente $(A$ e $B)$ para fechar um circuito e dois eletrodos para medir a diferença de potencial $(M$ e $N)$, conforme a Figura 7 . Na Figura 7 , 
o fluxo de corrente é no sentido de $A$ para $B$. Assim, partir da equação (6), os potenciais nos eletrodos $M$ e $N$ são dados por:

$$
\begin{gathered}
V_{M}=\frac{\rho I}{2 \pi}\left(\frac{1}{\overline{A M}}-\frac{1}{\overline{B M}}\right) \\
V_{N}=\frac{\rho I}{2 \pi}\left(\frac{1}{\overline{A N}}-\frac{1}{\overline{B N}}\right)
\end{gathered}
$$

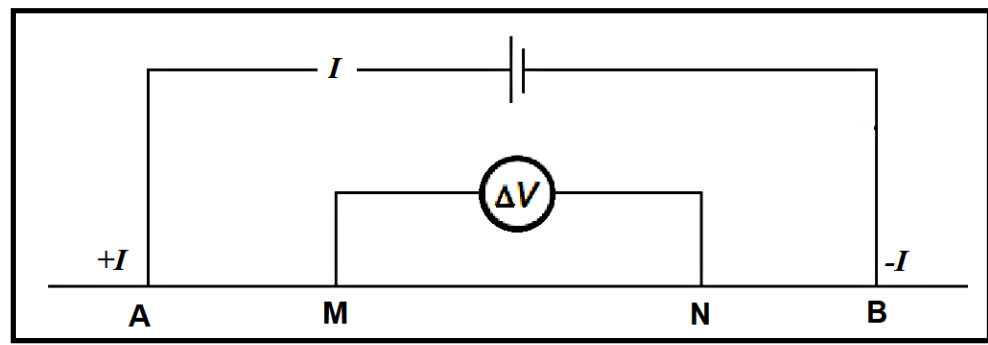

Figura 7 - Arranjo dos eletrodos de corrente (AB) e de potencial (MN) (adaptado de Kearey et al., 2002).

Portanto, a diferença de potencial entre $M$ e $N$ é dada por:

$$
\Delta V_{M N}=V_{M}-V_{N}=\frac{\rho I}{2 \pi}\left(\frac{1}{\overline{A M}}-\frac{1}{\overline{B M}}-\frac{1}{\overline{A N}}+\frac{1}{\overline{B N}}\right)
$$

Finalmente, a partir da equação (9) é possível obter a equação da resistividade aparente $\left(\rho_{a}\right)$ :

$$
\rho_{a}=K \frac{\Delta V}{I}
$$

onde $K$ representa o fator geométrico referente ao arranjo dos eletrodos na superfície dado por:

$$
K=2 \pi\left(\frac{1}{\overline{A M}}-\frac{1}{\overline{B M}}-\frac{1}{\overline{A N}}+\frac{1}{\overline{B N}}\right)^{-1}
$$

A profundidade de investigação depende da resistividade do meio e da separação dos eletrodos, ou seja, maiores profundidades são investigadas aumentando-se o tamanho do arranjo. 


\subsubsection{Técnicas e Arranjos de Aquisição}

$\mathrm{Na}$ aquisição dos dados de eletrorresistividade foram utilizadas duas técnicas: a Sondagem Elétrica Vertical (SEV) e o Caminhamento Elétrico (CE). A SEV é utilizada para determinar a variação da resistividade em função da profundidade (1D) e o CE é utilizado para investigar a variação lateral da resistividade em função da profundidade (2D). Existem vários tipos de arranjos de eletrodos, no entanto, na SEV foi utilizado o arranjo Schlumberger e no CE foram utilizados os arranjos dipolo-dipolo e polo-dipolo.

\section{Sondagem Elétrica Vertical - Arranjo Schlumberger}

Neste arranjo são utilizados quatro eletrodos, $A$ e $B$ para emitir a corrente e $M$ e $N$ para medir a diferença de potencial (Figura 8). O ponto de investigação é mantido fixo no centro do arranjo e aumenta-se gradativamente e simetricamente a distância dos eletrodos $A$ e $B$ em relação ao centro e, dessa forma, aumenta-se a profundidade de investigação. Os eletrodos $M$ e $N$ ficam mais próximos do centro e não é necessário movimentá-los a cada medida, somente quando o sinal de leitura se torna fraco ou ruidoso. Neste caso é possível aumentar o potencial aumentando-se a separação de $M$ e $N$ e mantendo-se fixos os eletrodos $A$ e $B$ para uma mesma medida, sendo este processo conhecido como embreagem.

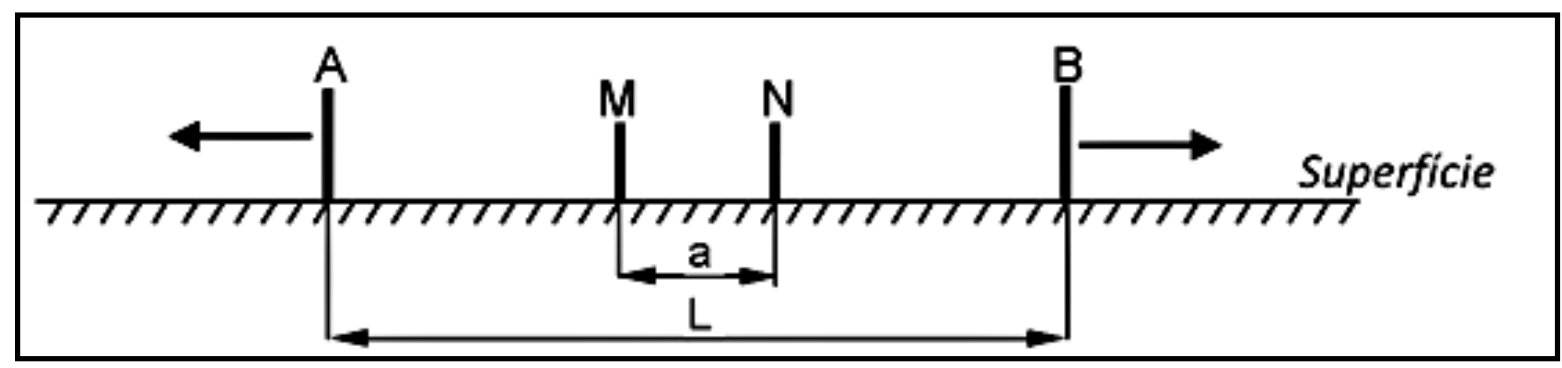

Figura 8 - Sondagem elétrica vertical - arranjo Schlumberger (adaptado de Telford et al., 1990).

\section{Caminhamento Elétrico - Arranjo dipolo-dipolo}

No caminhamento elétrico com o arranjo dipolo-dipolo, o espaçamento $a$ entre os eletrodos de corrente $A$ e $B$ e dos eletrodos de potencial $M$ e $N$ são iguais (Figura 9). Utiliza-se simultaneamente vários ( $n$ ) pares de eletrodos (dipolos) de potencial $M$ e $N$ dispostos ao longo do perfil e cada dipolo $M$ e $N$ corresponde a um nível de investigação em profundidade. Portanto, quanto maior o número $(n)$ de dipolos $M$ e $N$, maior a profundidade de investigação. O espaçamento $a$ é mantido fixo e os pares de eletrodos são movidos progressivamente a cada 
medida ao longo do perfil. Este arranjo apresenta uma boa resolução lateral, porém, conforme aumenta-se o valor de $n$, a intensidade do sinal diminui. Nesta pesquisa foram utilizados seis níveis de investigação $(n=6)$.

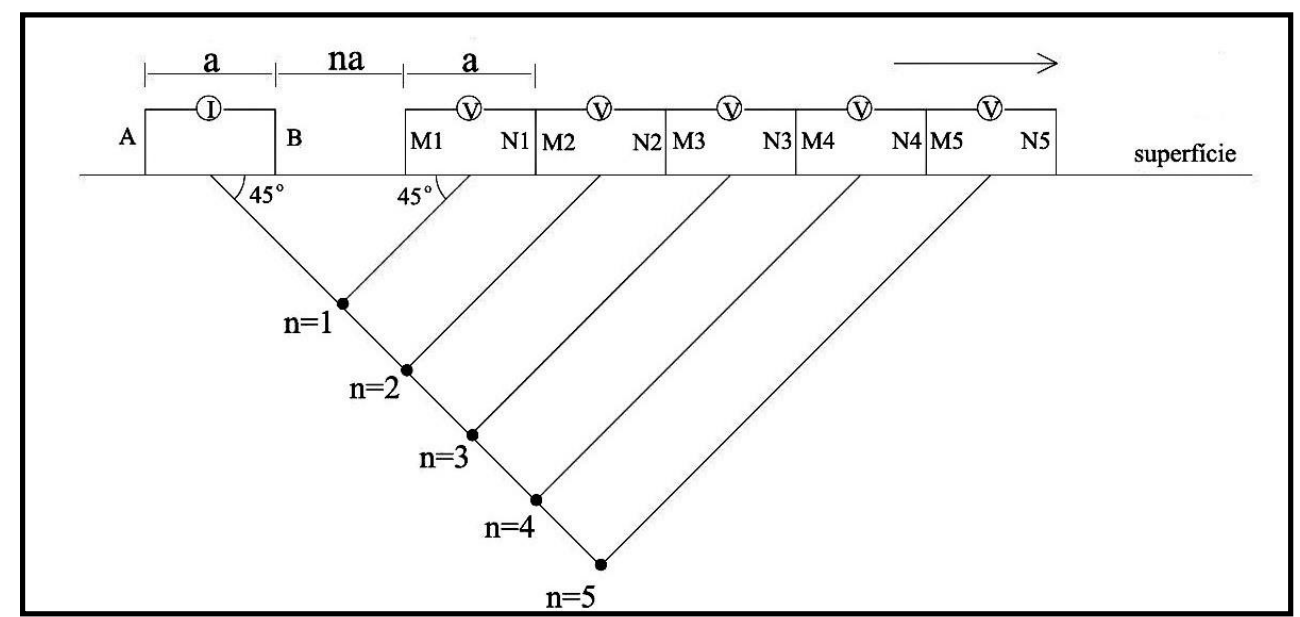

Figura 9 - Caminhamento elétrico - arranjo dipolo-dipolo (adaptado de Telford et al., 1990).

\section{Caminhamento Elétrico - Arranjo polo-dipolo}

O arranjo polo-dipolo é similar ao dipolo-dipolo, a diferença é que o eletrodo de corrente $B$ permanece fixo a uma distância de 10 a 20 vezes o espaçamento $a$ dos eletrodos de potencial $M$ e $N$ (Figura 10). O espaçamento $a$ entre os eletrodos de potencial é constante e o eletrodo de corrente $A$ fica a uma distância $n \cdot a$ dos eletrodos de potencial. Neste caso também, quanto maior o número $(n)$ de dipolos $M$ e $N$, maior a profundidade de investigação. Comparado ao arranjo dipolo-dipolo, a intensidade do sinal e, consequentemente, a profundidade de investigação são maiores, porém, a resolução horizontal é menor. Para este arranjo também foram utilizados seis níveis de investigação $(n=6)$.

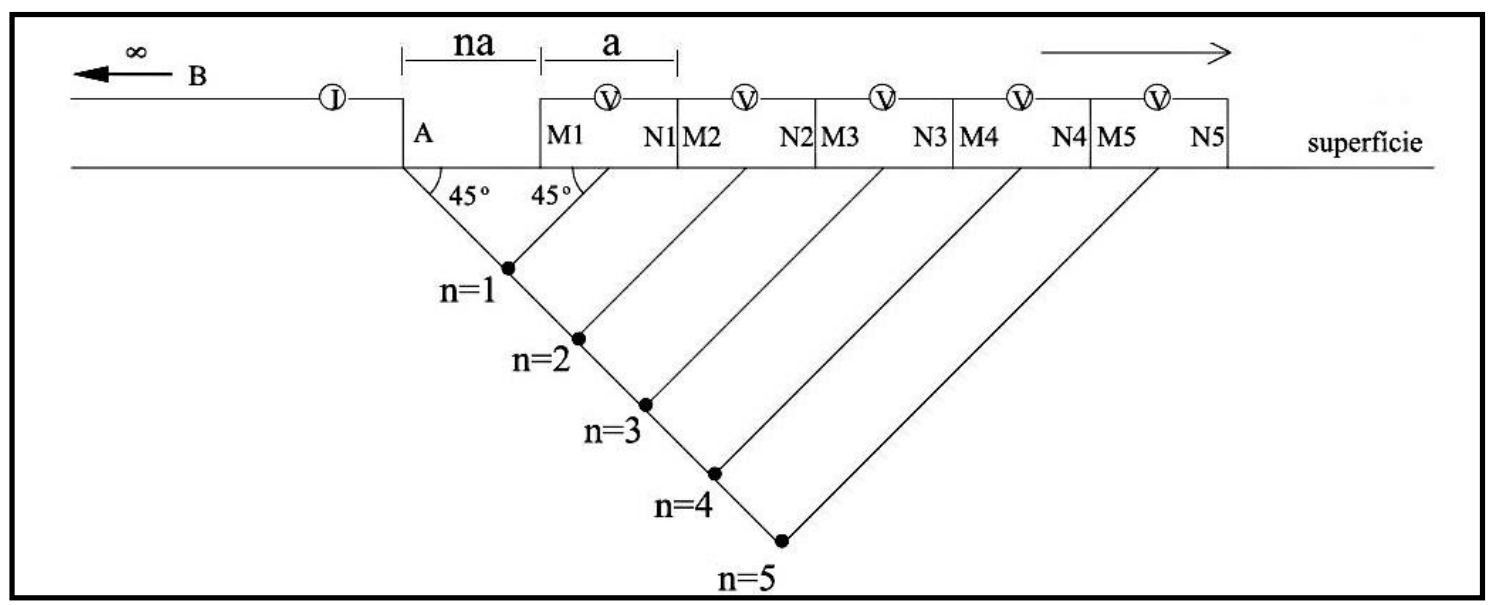

Figura 10 - Caminhamento elétrico - arranjo polo-dipolo (adaptado de Telford et al., 1990). 


\subsection{Método Eletromagnético no Domínio do Tempo (TDEM)}

O método TDEM baseia-se no princípio de indução eletromagnética e é utilizado para estimar a variação da resistividade em função da profundidade. A investigação é realizada através da medida do decaimento de um campo magnético secundário induzido na subsuperfície devido à variação de um campo magnético primário gerado na superfície.

\subsubsection{Princípios Físicos do Método TDEM}

A maioria dos métodos eletromagnéticos pode ser classificada como pertencentes ao domínio da frequência ou ao domínio do tempo. Nos Métodos Eletromagnéticos no Domínio da Frequência (FDEM), o campo magnético secundário, induzido nos materiais em subsuperfície, é medido na presença do campo magnético primário, gerado pelo equipamento ou pela própria fonte natural (no caso do Método Magnetotelúrico (MT)). O campo primário é muito maior que o secundário, por isso, a remoção do campo primário do sinal medido pode gerar uma perda do campo secundário e, com isso, reduzir a precisão dos resultados. O método TDEM não apresenta essa limitação, pois as medidas do campo secundário são realizadas sem a presença do campo primário.

O método TDEM empregado nesta pesquisa consiste em dispor um loop transmissor quadrado e uma bobina receptora, ambos na superfície (Figura 11). Inicialmente injeta-se uma corrente continua no loop transmissor. Em seguida essa corrente é desligada e, no intervalo de tempo da ordem de microssegundos que a corrente vai a zero, o campo eletromagnético primário transiente induz um campo eletromagnético secundário transiente nos materiais em subsuperfície (Figura 12). A variação do campo magnético secundário é então medida pela bobina receptora.

A Figura 11 apresenta um esquema do loop transmissor e da bobina receptora e as correntes secundárias associadas ao campo secundário. Inicialmente, as correntes induzidas apresentam as mesmas dimensões do loop transmissor e, conforme são difundidas na subsuperfície, suas dimensões aumentam, num efeito conhecido como anéis de fumaça (smoke rings). As correntes secundárias sofrem atenuação conforme vão se aprofundando devido ao efeito Joule, onde a energia se converte em calor.

O método TDEM é sensível às camadas de baixa resistividade (condutoras), por causa da maior quantidade de fluxos de corrente que são induzidas nessas camadas. Quanto mais 
resistivo for o meio, mais rápido o campo secundário se difunde e, portanto, maior a penetração na subsuperfície. Por outro lado, quanto mais condutivo for o meio, mais lentamente o campo secundário se difunde e, consequentemente, menor a penetração na subsuperfície.

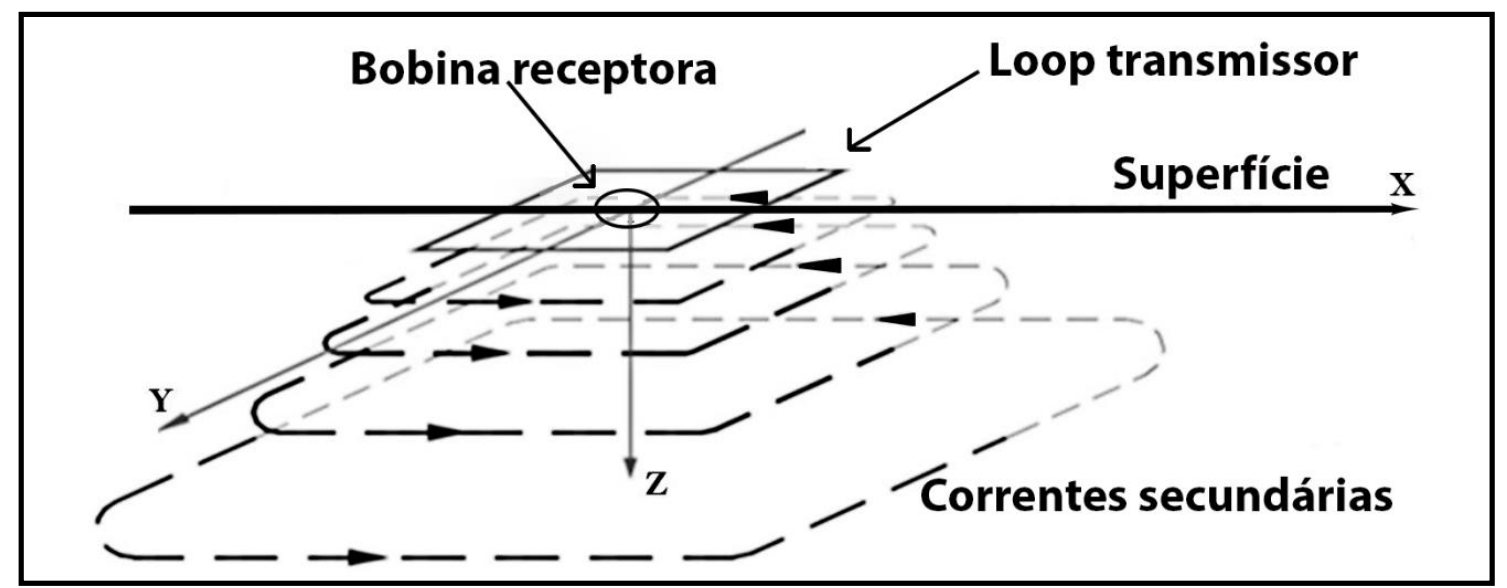

Figura 11 - Esquema do loop transmissor, bobina receptora e as correntes secundárias induzidas na subsuperfície (adaptado de McNeill, 1994).

A Figura 12 apresenta a sequência de eventos do método TDEM. Durante uma aquisição esse processo é repetido por vários ciclos e os resultados são empilhados para aumentar a relação sinal/ruído. A corrente elétrica gerada no loop transmissor é na forma de pulsos alternados para evitar a polarização do meio e a medida da variação do campo secundário é feita de modo discretizado em intervalos de medida ou janelas (gates) de aquisição, que variam de acordo com o equipamento receptor.

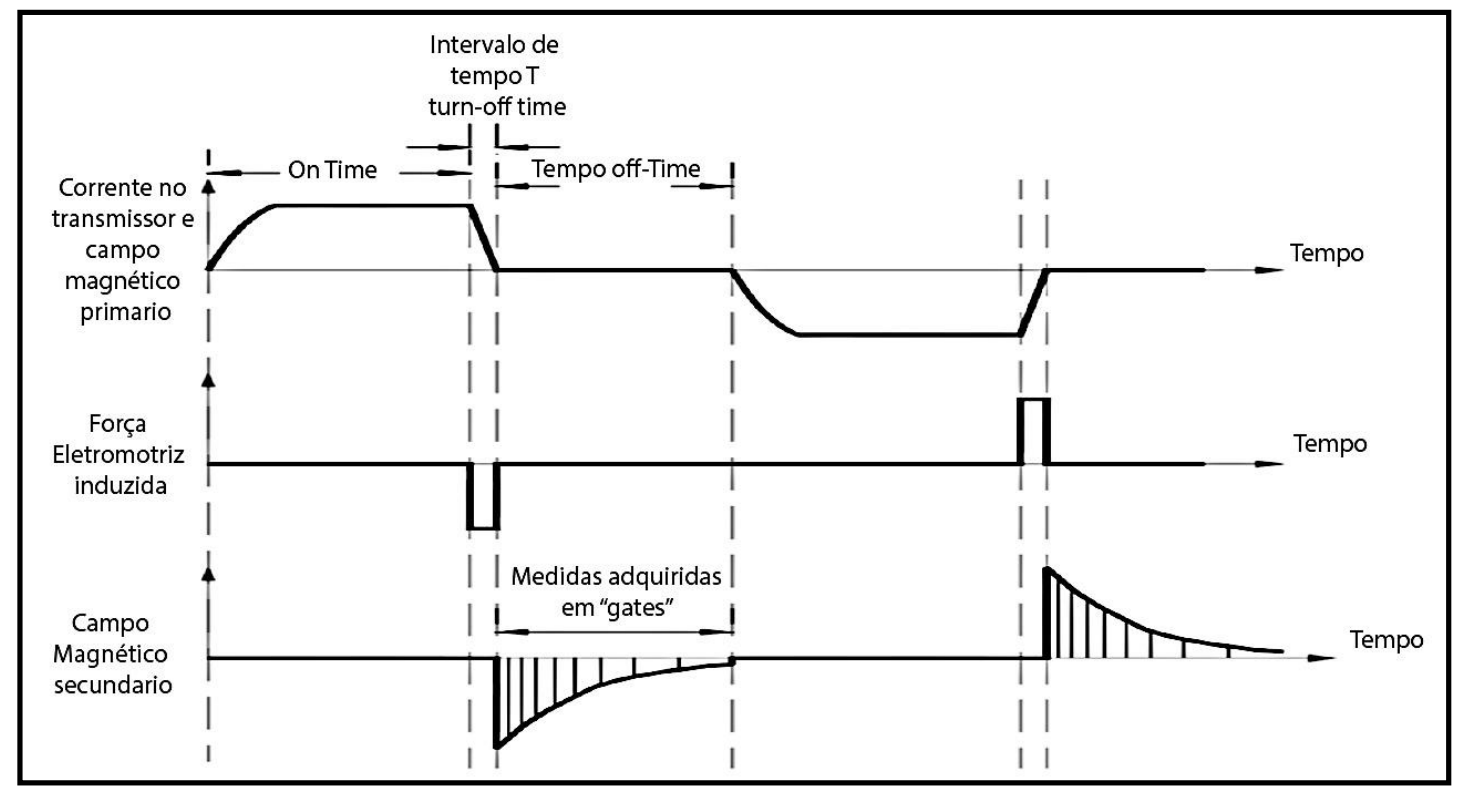

Figura 12 - Sequência de eventos do método TDEM (adaptado de Christiansen et al., 2006). 
Christiansen et al. (2006) demonstraram a relação entre a variação do campo magnético secundário em função do tempo $(\partial B / \partial t)$ e a resistividade aparente $\left(\rho_{a}\right)$ conforme exposto a seguir.

Um campo eletromagnético é definido por cinco funções vetoriais: $\boldsymbol{E}$ (campo elétrico $(\mathrm{V} / \mathrm{m})), \boldsymbol{B}$ (indução magnética $\left(\mathrm{Wb} / \mathrm{m}^{2}=\mathrm{T}\right)$ ), $\boldsymbol{D}$ (densidade de fluxo elétrico $\left(\mathrm{C} / \mathrm{m}^{2}\right)$ ), $\boldsymbol{H}$ (campo magnético $(\mathrm{A} / \mathrm{m}))$ e $\boldsymbol{J}\left(\right.$ densidade de corrente elétrica $\left.\left(\mathrm{A} / \mathrm{m}^{2}\right)\right)$. A relação entre essas funções é descrita pelas equações de Maxwell dadas por:

$$
\begin{aligned}
& \Delta \times \boldsymbol{E}+\frac{\partial \boldsymbol{B}}{\partial t}=0 \\
& \Delta \times \boldsymbol{H}-\frac{\partial \boldsymbol{D}}{\partial t}=\boldsymbol{J}
\end{aligned}
$$

$$
\Delta \cdot \boldsymbol{B}=0
$$

$$
\Delta \cdot \boldsymbol{D}=q
$$

onde $q$ é a densidade volumétrica de carga $\left(\mathrm{C} / \mathrm{m}^{3}\right)$.

As relações constitutivas entre os vetores são:

$$
\begin{aligned}
& \boldsymbol{J}=\sigma \boldsymbol{E} \\
& \boldsymbol{D}=\varepsilon \boldsymbol{E} \\
& \boldsymbol{B}=\mu \boldsymbol{H}
\end{aligned}
$$

onde $\sigma$ é a condutividade elétrica, $\varepsilon$ a permissividade dielétrica e $\mu$ é a permeabilidade magnética do meio (assumida como sendo igual à do vácuo $\mu_{0}$ ).

Aplicando a transformada de Fourier nas equações (12) e (13) e utilizando as equações (16) a (18), obtém-se as equações de Maxwell no domínio da frequência:

$$
\Delta \times \boldsymbol{E}+i \omega \mu_{0} \boldsymbol{H}=0
$$




$$
\Delta \times \boldsymbol{H}-(\sigma+i \varepsilon \omega) \boldsymbol{E}=0
$$

onde $\omega$ é a frequência angular.

Por conveniência, são utilizadas as grandezas impeditividade $(\hat{z}=i \omega \mu 0) \mathrm{e}$ admissividade $(\hat{y}=\sigma+i \varepsilon \omega)$. As equações (19) e (20) são aplicadas apenas para regiões livres de cargas e, para regiões que contêm cargas, elas são substituídas pelas seguintes equações não homogêneas:

$$
\begin{gathered}
\Delta \times \boldsymbol{E}+\hat{z} \boldsymbol{H}=-\boldsymbol{J}_{m}^{s} \\
\Delta \times \boldsymbol{H}+\hat{y} \boldsymbol{E}=-\boldsymbol{J}_{e}^{s}
\end{gathered}
$$

onde $\boldsymbol{J}_{m}^{S}$ é a corrente de fonte magnética e $\boldsymbol{J}_{e}^{S}$ é a corrente de fonte elétrica.

As equações não homogêneas (21) e (22) podem ser resolvidas para regiões homogêneas se $\boldsymbol{J}_{m}^{S}$ e $\boldsymbol{J}_{e}^{S}$ podem ser definidas. Expressando $\boldsymbol{E}$ e $\boldsymbol{H}$ em termos dos potenciais de Schelkunoff $\boldsymbol{A}$ e $\boldsymbol{F}$, facilita a derivação de $\boldsymbol{E}$ e $\boldsymbol{H}$ por diferenciação. A solução das equações fica mais fácil porque os potenciais $\boldsymbol{A}$ e $\boldsymbol{F}$ são paralelos às fontes de corrente, ao contrário dos campos $\boldsymbol{E}$ e $\boldsymbol{H}$. De modo geral, os campos elétrico e magnético em cada região homogênea são descritos como uma superposição de fontes elétricas ou magnéticas da seguinte forma:

$$
\begin{gathered}
E=E_{m}+E_{e} \\
H=H_{m}+H_{e}
\end{gathered}
$$

Assim, um campo eletromagnético é descrito pelos pares de campos $\left(\boldsymbol{E}_{\boldsymbol{m}}, \boldsymbol{H}_{\boldsymbol{m}}\right)$ e $\left(\boldsymbol{E}_{\boldsymbol{e}}\right.$, $\boldsymbol{H}_{\boldsymbol{e}}$ ). Para o primeiro par assume-se $\boldsymbol{J}_{e}^{S}$ como zero e, para o segundo, $\boldsymbol{J}_{m}^{S}$ como zero, ou seja, a fonte de corrente elétrica é nula para os campos elétricos e magnéticos gerados numa fonte magnética e vice-versa.

O método TDEM utiliza um dipolo magnético vertical para induzir campos elétricos horizontais. Assim, a situação se torna mais simples porque é necessário apenas a utilização do potencial $\boldsymbol{F}$ de Schelkunoff. Para materiais geológicos e frequências menores que $10^{5} \mathrm{~Hz}$, a corrente de deslocamento se torna desprezível em comparação à corrente de condução, sendo esta aproximação chamada de "quase-estática". Dessa forma, os campos elétrico e magnético 
totais originados pela fonte magnética podem ser derivados usando o potencial $\boldsymbol{F}$, obtendo-se:

$$
\begin{gathered}
\boldsymbol{E}_{\boldsymbol{m}}=-\Delta \times \boldsymbol{F} \\
\boldsymbol{H}_{\boldsymbol{m}}=-\hat{y} \boldsymbol{F}+\frac{1}{\hat{z}} \Delta(\Delta \cdot \boldsymbol{F})
\end{gathered}
$$

Considerando um meio estratificado unidimensional, $\boldsymbol{F}$ consiste apenas na componente vertical. Para o método TDEM, o campo transmitido é um campo elétrico transversal gerado pelo loop transmissor. Dessa forma, o campo magnético vertical no centro do loop pode ser calculado como uma integração de dipolos magnéticos verticais sobre a sua área. Assim, segundo Christiansen et al. (2006), o potencial $\boldsymbol{F}$ pode ser entendido como um dipolo magnético vertical, dado por:

$$
F(r, z)=\frac{\widehat{z_{0}} m}{4 \pi} \int_{0}^{\infty}\left[e^{-u_{0}|z+h|}+r_{T E} e^{u_{0}(z-h)}\right] \frac{\lambda}{u_{0}} J_{0}(\lambda r) d \lambda
$$

onde $m$ é o momento magnético de dipolo, $J_{0}$ é a função de Bessel de ordem zero, $\lambda=$ $\sqrt{k_{x}^{2}+k_{y}^{2}}$, sendo $k_{x}$ e $k_{y}$ as frequências espaciais nas direções $x$ e $y, u_{n}=\sqrt{\lambda^{2}-k_{n}^{2}}$, onde $k_{n}$ é o número de onda da camada $n$, que na aproximação quase-estática é igual a $k_{n}^{2}=$ $-i \mu_{0} \sigma_{n} \omega, r=\sqrt{x^{2}+y^{2}}$ é a distância radial da fonte ao receptor e $r_{T E}$ é definido como o coeficiente de reflexão.

Integrando a equação (27) sobre um loop circular de raio $a$ e uma corrente elétrica $I$, obtém-se:

$$
F(r, z)=\frac{\widehat{z_{0}} I a}{2} \int_{0}^{\infty} \frac{1}{u_{0}}\left[e^{-u_{0}|z+h|}+r_{T E} e^{u_{0}(z-h)}\right] J_{1}(\lambda a) J_{0}(\lambda r) d \lambda
$$

onde $J_{1}$ é a função de Bessel de primeira ordem. Substituindo a equação (28) na equação (26) e assumindo o receptor no centro do loop transmissor, obtém-se a componente vertical do campo magnético na forma:

$$
H_{z}=\frac{I a}{2} \int_{0}^{\infty}\left[e^{-u_{0}|z+h|}+r_{T E} e^{u_{0}(z-h)}\right] \frac{\lambda^{2}}{u_{0}} J_{1}(\lambda a) d \lambda
$$


A equação (29) está no domínio da frequência e a resposta no domínio do tempo é obtida a partir da transformada inversa de Laplace ou de Fourier. A equação (29) não pode ser resolvida analiticamente devido a sua complexidade, porém, considerando o receptor no centro do loop transmissor e um meio homogêneo, $r_{T E}$ pode ser expresso como:

$$
r_{T E}=\frac{\lambda-u}{\lambda+u}
$$

Assim, a equação (29) simplifica para:

$$
H_{z}=I a \int_{0}^{\infty} \frac{\lambda^{2}}{\lambda-u} J_{1}(\lambda a) d \lambda
$$

Resolvendo a integral e aplicando a transformada inversa de Laplace na equação (31) obtém-se:

$$
B_{z}=\frac{\mu_{0} I}{2 a}\left[\frac{3}{\pi^{1 / 2} \theta a} e^{-\theta^{2} a^{2}}+\left(1-\frac{3}{2 \theta^{2} a^{2}}\right) \operatorname{erf}(\theta a)\right]
$$

onde $\theta=\sqrt{\mu_{0} \sigma / 4 t}$ e $\operatorname{erf}(\theta a)$ é a função erro.

A partir da equação (32), fazendo $t$ tender a zero, $B_{z}=\mu_{0} I / 2 a$, sendo essa a intensidade do campo magnético primário antes da corrente ser desligada no loop transmissor.

Derivando a equação (32) em função do tempo, obtém-se:

$$
\frac{\partial B_{z}}{\partial t}=-\frac{I}{\sigma a^{3}}\left[3 \operatorname{erf}(\theta a)-\frac{2}{\pi^{\frac{1}{2}}} \theta a\left(3+2 \theta^{2} a^{2}\right) e^{-\theta^{2} a^{2}}\right]
$$

A equação (33) representa a variação do campo magnético secundário em função do tempo que é medido pela bobina receptora. No entanto, as medidas são feitas após um pequeno intervalo de tempo transcorrido do desligamento da corrente (aproximadamente 100 microssegundos), quando o campo primário já não está mais presente. Assim, após esse intervalo de tempo, a equação (33) é aproximada por: 


$$
\frac{\partial B_{z}}{\partial t} \approx\left(\frac{I \pi a^{2}}{20}\right)\left(\frac{\sigma}{\pi}\right)^{3 / 2}\left(\frac{\mu_{0}}{t}\right)^{5 / 2}
$$

Portanto, a resistividade aparente para método TDEM é dada pela expressão:

$$
\rho_{a}=\frac{1}{\pi}\left(\frac{I \pi a^{2}}{20 \partial B_{z} / \partial t}\right)^{2 / 3}\left(\frac{\mu_{0}}{t}\right)^{5 / 3}
$$

\subsubsection{Profundidade de Investigação}

O conceito de skin depth usado nos métodos eletromagnéticos no domínio da frequência, também pode ser utilizado no método TDEM. O skin depth representa a profundidade de investigação que o campo eletromagnético gerado decai a $1 / e$ do seu valor inicial. Segundo Spies (1989), o skin depth no domínio do tempo é dado por:

$$
\delta_{T D}=\sqrt{\frac{2 t}{\mu \sigma}} \approx 1,26 \sqrt{\rho t}
$$

onde $\mu$ é a permeabilidade magnética, $\sigma$ é a condutividade, $\rho$ é a resistividade e $t$ é o tempo de aquisição em segundos.

Ainda de acordo com Spies (1989), a profundidade de investigação para o método TDEM é dada por:

$$
d \approx 0,55\left(\frac{I A}{\sigma \eta_{V}}\right)^{1 / 5}
$$

onde $I$ é a corrente injetada no loop transmissor, $A$ é a área do loop transmissor e $\eta_{V}$ é o nível de ruído na bobina receptora, onde, assumindo-se um nível de ruído típico igual a $0,5 \mathrm{nV} / \mathrm{m}^{2}$, obtém-se:

$$
d \approx 40(M \rho)^{1 / 5}
$$

onde $M=I A$ é o momento de dipolo magnético do transmissor. Portanto, aumentando-se o 
momento de dipolo é possível atingir maiores profundidades de investigação.

As aquisições dos dados TDEM foram realizadas com uma corrente $(I)$ entre 17,5 A e 18,0 A e um loop transmissor quadrado com $200 \mathrm{~m}$ de lado, ou seja, uma área de $40.000 \mathrm{~m}^{2}$. Assim, a partir da equação (38), assumindo um meio com resistividade média de $30 \Omega \cdot \mathrm{m}$, a profundidade de investigação teórica é de aproximadamente $1166 \mathrm{~m}$.

\subsubsection{Ruídos e Acoplamentos}

O método TDEM é pouco suscetível à influência de ruídos provenientes de fontes naturais comparado com outros métodos eletromagnéticos como, por exemplo, o magnetotelúrico. As principais fontes de ruído são artefatos produzidos pelo homem, como linhas de transmissão de energia, cabos e tubulações enterradas e cercas metálicas próximas a área de aquisição de dados. Sørensen et al. (2000) classificaram as fontes de acoplamento do método TDEM em duas formas: o galvânico e o capacitivo (Figura 13).

O acoplamento galvânico ocorre quando há um condutor em contato galvânico com o solo como, por exemplo, linhas de transmissão de energia com torres aterradas. $\mathrm{O}$ transmissor do TDEM induz correntes elétricas nestes condutores aterrados que, por conseguinte, interferem nas correntes secundárias induzidas nos materiais em subsuperfície. Este sistema caracteriza um circuito indutor-resistor (LR) que apresenta um decaimento exponencial, como é apresentado na Figura 13a. O campo magnético relacionado à fonte de interferência encontra-se em fase com o campo magnético secundário, resultando em um deslocamento da curva de decaimento, podendo ser confundido com uma camada rasa de baixa resistividade. Este tipo de acoplamento não pode ser removido através de nenhuma filtragem, porém, pode ser identificado quando há dados de sondagens próximas ao local onde o efeito foi detectado.

O acoplamento capacitivo ocorre quando há um material metálico envolto por um isolante, como por exemplo, um cabo elétrico no subsolo. Este sistema caracteriza um circuito resistor-indutor-capacitor (RLC), como é apresentado na Figura 13b. O transmissor carrega o capacitor do circuito que é então descarregado de forma oscilante. Desta forma, a resposta deste acoplamento é o decaimento oscilante das correntes induzidas, sendo facilmente identificável nos dados. Neste caso também não é possível remover o efeito dos dados, mas, por ser facilmente detectado, pode ser evitado mudando-se a área de aquisição de dados para uma área neutra a este tipo de acoplamento. Vale ressaltar que os dados TDEM adquiridos em Taubaté não foram afetados por nenhuma dessas fontes de acoplamentos. 


\section{a) Acoplamento Galvânico}
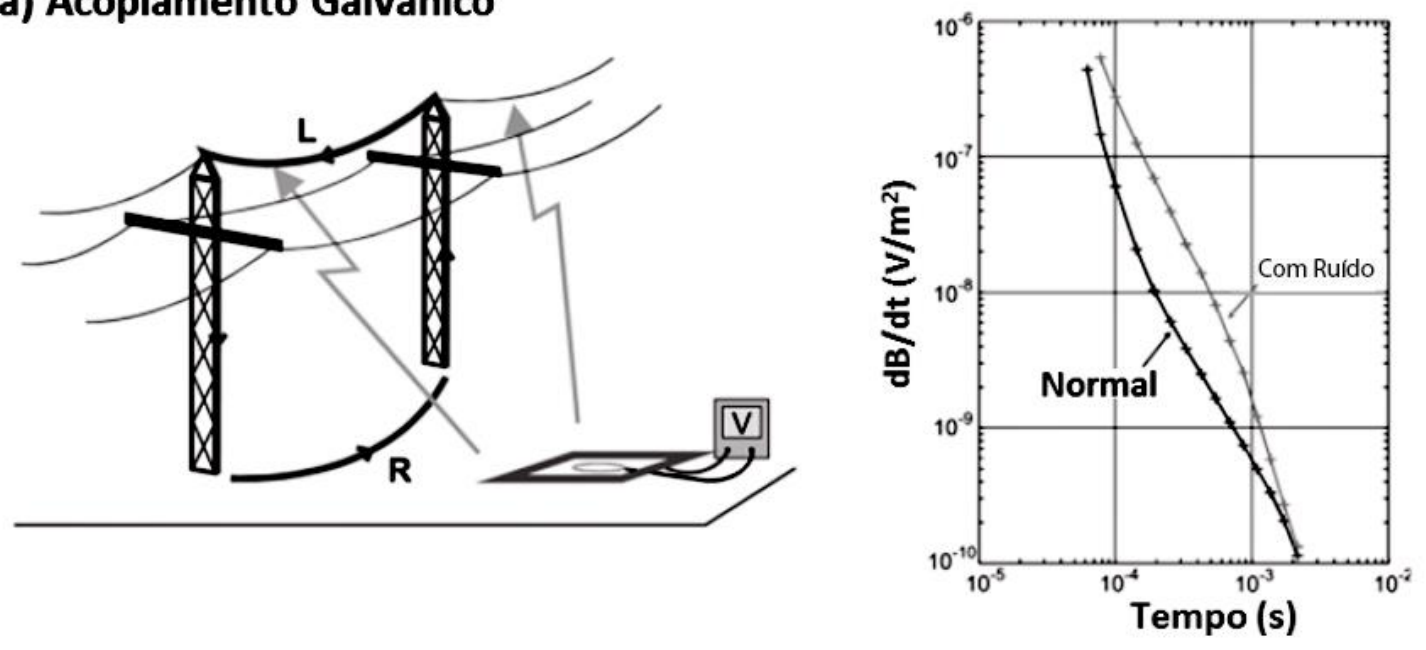

\section{b) Acoplamento Capacitivo}

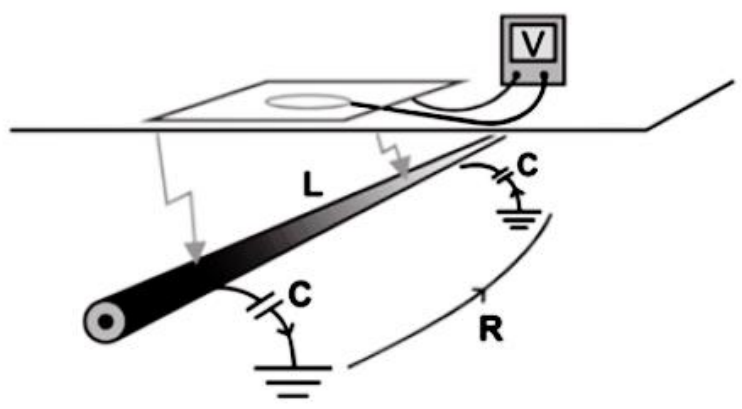

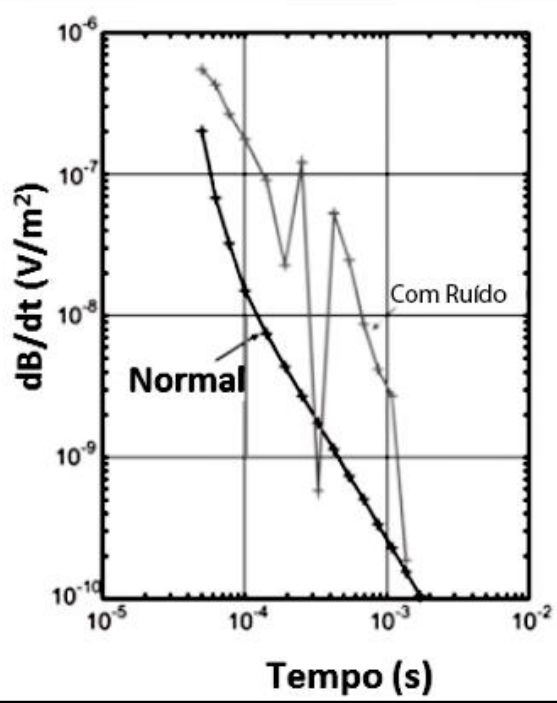

Figura 13 - Fontes de acoplamentos do método $\operatorname{TDEM}(\mathrm{R}=$ resistor, $\mathrm{L}=$ indutor e $\mathrm{C}=$ capacitor $)$ : a) Acoplamento galvânico; b) Acoplamento capacitivo (adaptado de Sørensen et al., 2000).

\subsubsection{Arranjos de Aquisição}

Existem vários tipos de arranjos de aquisição e procedimentos de campo no método TDEM. A escolha da configuração do arranjo e dos parâmetros de aquisição dependem do objetivo da pesquisa.

Assim como no método ER, no método TDEM é possível investigar as variações vertical e lateral da resistividade elétrica dos materiais em subsuperfície. Na aquisição dos dados TDEM foram utilizados dois tipos de arranjos: o loop central (1D) e o caminhamento TDEM com loop transmissor fixo e a bobina receptora móvel (2D).

No arranjo loop central (Figura 14a) a bobina receptora é posicionada no centro do loop transmissor. Para uma bobina receptora com $1 \mathrm{~m}$ de diâmetro, o loop transmissor não 
pode ter menos que $40 \mathrm{~m}$ de lado. Esse arranjo é um dos mais utilizados no método TDEM e tem a vantagem de apresentar uma boa relação sinal/ruído.

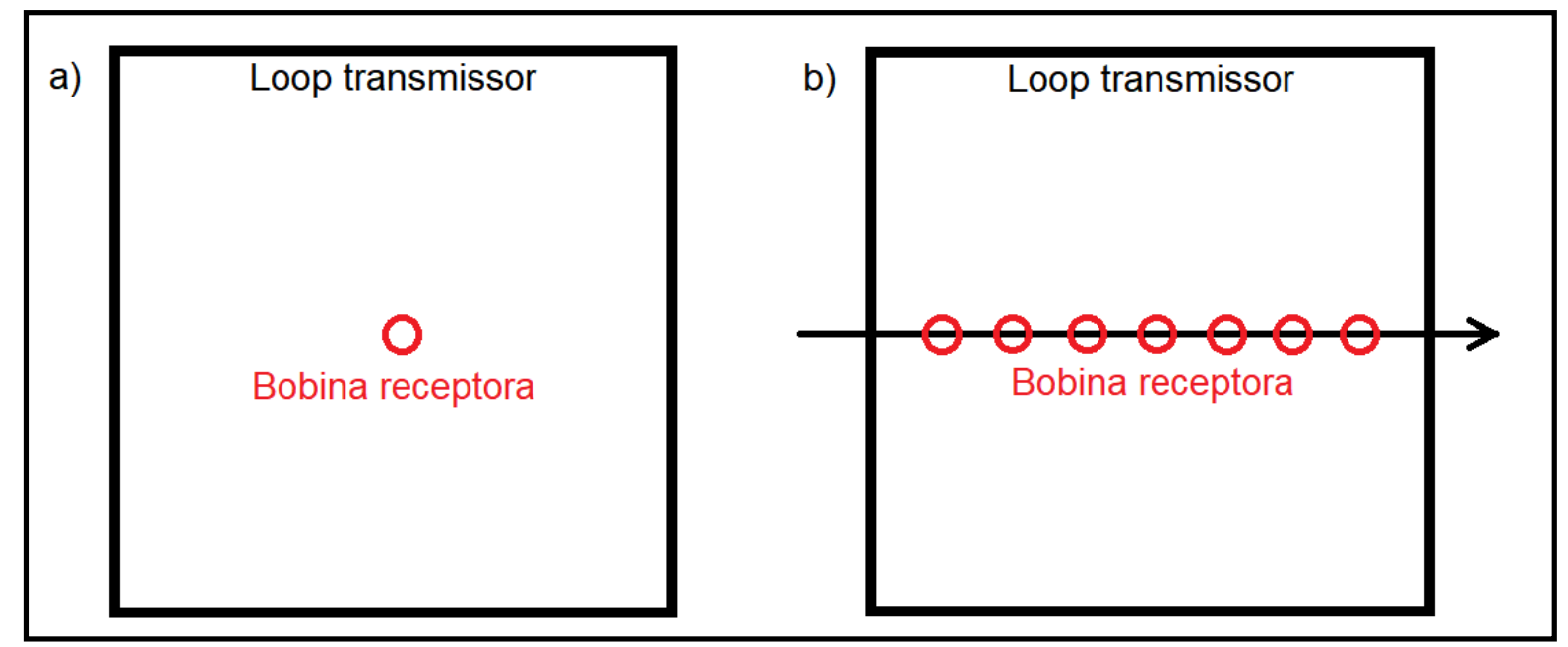

Figura 14 - Arranjos de aquisição TDEM: a) Loop central e b) Caminhamento TDEM com loop transmissor fixo e a bobina receptora móvel.

No caminhamento TDEM com loop transmissor fixo e a bobina receptora móvel (Figura 14b), a bobina receptora é movida com um espaçamento fixo ao longo de uma linha que atravessa o loop transmissor, formando um perfil. As sondagens são realizadas apenas no interior do loop transmissor, pois, assumindo-se que as linhas do campo magnético são positivas saindo da terra em direção ao ar, quando a bobina receptora é colocada do lado de fora do loop transmissor, a premissa do campo magnético positivo é violada e obtêm-se valores negativos de resistividade aparente (Bortolozo et al., 2016). Além disso, quanto mais distante a bobina receptora é posicionada do lado de fora do loop transmissor, mais fraco e ruidoso é o sinal medido. 


\section{INVERSÃO DOS DADOS}

Este capítulo consiste em um resumo da metodologia de Inversão Individual e Conjunta de dados de CE e CTDEM desenvolvida por Bortolozo (2011 e 2016).

\subsection{Inversão em Geofísica}

A inversão de dados geofísicos pode ser dividida em três etapas:

1) formulação do problema e determinação de um modelo interpretativo;

2) obtenção dos parâmetros que definem esse modelo e

3) avaliação do modelo obtido.

O objetivo da primeira etapa é determinar um modelo interpretativo capaz de representar as principais características da subsuperfície. Deve-se considerar a propriedade física investigada e a dimensão do problema (1D, 2D, etc). O modelo interpretativo é definido por parâmetros conhecidos, normalmente relacionados aos parâmetros de aquisição dos dados, e parâmetros desconhecidos, que no caso dos métodos geoelétricos são as resistividades e as espessuras das camadas.

O objetivo da segunda etapa é obter os parâmetros que propiciam o ajuste aos dados medidos e, simultaneamente, satisfazem vínculos geológicos eventualmente disponíveis, sendo esta a inversão dos dados propriamente dita. Esse processo varia caso a caso, pois existem diversos algoritmos de inversão.

A terceira e última etapa consiste em analisar a confiabilidade dos parâmetros obtidos na inversão, onde é fundamental a atuação do intérprete para determinar a coerência entre o modelo obtido e os parâmetros geológicos conhecidos da área de estudo.

$\mathrm{Na}$ inversão conjunta os dados de duas ou mais metodologias são invertidos simultaneamente, ou seja, o mesmo modelo deve satisfazer todas as bases de dados, aumentando-se assim a confiança do resultado obtido.

\subsection{Modelagem Numérica}

$\mathrm{Na}$ geofísica, a modelagem numérica consiste em simular matematicamente a distribuição espacial de uma determinada propriedade física a partir de um modelo representativo do meio geológico. 
No caso dos métodos Eletrorresistivo (ER) 2D e Eletromagnético no Domínio do Tempo (TDEM) 2D, o modelo geoelétrico é representado por uma seção discretizada em polígonos, onde cada polígono possui um valor de resistividade elétrica independente dos demais. Desse modo, o problema direto consiste em determinar a distribuição dos campos elétricos e eletromagnéticos na seção discretizada devido a uma fonte conhecida. Existem diversas formas de discretizar o meio, no entanto, uma das mais utilizadas em métodos elétricos e eletromagnéticos é a aproximação por diferenças finitas. A vantagem desse método é a sua capacidade em discretizar o meio para a maioria das estruturas de forma irregular e arbitrária. Além disso, é possível reduzir um problema contínuo, com um número infinito de variáveis, em um problema discreto, com um número finito de variáveis, possibilitando assim resolver o problema computacionalmente.

\subsubsection{Modelagem ER 2D por Diferenças Finitas}

A modelagem ER 2D foi realizada com base no artigo de Dey e Morrison (1979), onde os autores simularam uma distribuição de condutividade elétrica em um semi-plano. Para isso é necessário definir uma malha para a discretização, que consiste em um conjunto finito de pontos ou nós em um semi-plano. A Figura 15 apresenta uma malha cartesiana ortogonal uniforme onde qualquer ponto $(x, y)$ é representado na malha por $(i, j)$ e os seus vizinhos por $(i \pm 1, j \pm 1)$. Desse modo as derivadas são aproximadas pelas diferenças dos valores numéricos da função do potencial elétrico nestes pontos.

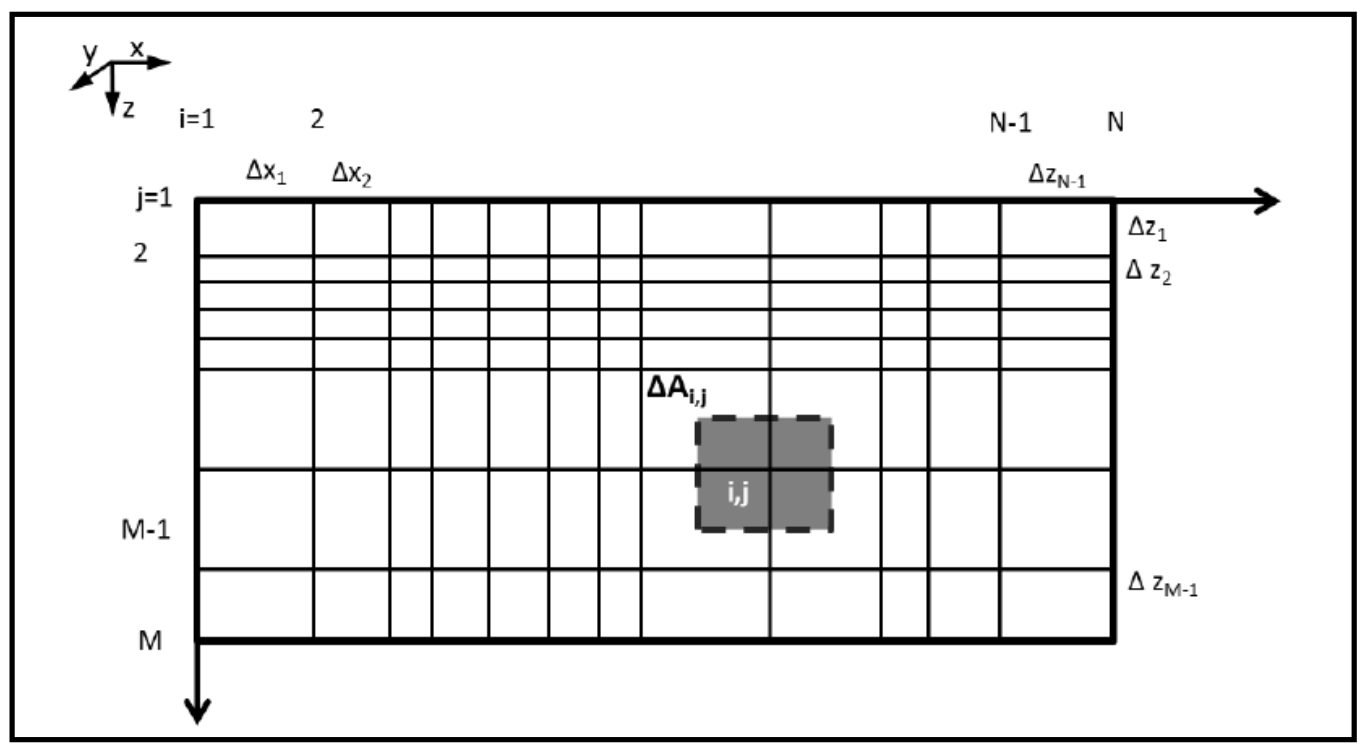

Figura 15 - Discretização da eletrorresistividade 2D por diferenças finitas. $\Delta A_{i, j}$ representa um elemento de área discretizada (Bortolozo, 2016). 
Pela lei de Ohm, a densidade de corrente $J$ é proporcional a intensidade do campo elétrico $E$ e uma condutividade elétrica isotrópica $\sigma$, dada por:

$$
\bar{J}=\sigma \bar{E}
$$

Como os campos elétricos estacionários são conservativos, é possível aplicar o princípio da conservação de carga ao longo de um volume. Assim, a partir da equação de continuidade, obtém-se:

$$
\Delta \cdot \bar{J}=\frac{\partial \rho}{\partial t} \delta(x) \delta(y) \delta(z)
$$

onde $\rho$ é a densidade de carga definida em um ponto $(x, y, z)$ por uma função delta de Dirac.

Assume-se que não há mudança na distribuição de condutividade no eixo y (strike geoelétrico) e, para facilitar o cálculo, aplica-se a transformada de Fourier, transformando $y$ em $K y$. Assim, para um valor fixo de $K y$, a distribuição tridimensional do potencial elétrico $\phi(x, y, z)$ devido a uma fonte pontual em $\left(x_{S}, y_{S}, z_{S}\right)$ sobre uma distribuição bidimensional de condutividade $\sigma(x, z)$, é reduzida para o potencial transformado bidimensional $\tilde{\phi}(x, K y, z)$ :

$$
\begin{gathered}
\nabla^{2}\{\sigma(x, z) . \nabla \tilde{\phi}(x, K y, z)\}+\sigma(x, z) \nabla^{2} \tilde{\phi}(x, K y, z)-\tilde{\phi}(x, K y, z) \nabla^{2} \sigma(x, z) \\
-2 K y^{2} \sigma(x, z) \tilde{\phi}(x, K y, z)=-2 \tilde{Q} \delta\left(x_{s}\right) \delta\left(z_{s}\right)
\end{gathered}
$$

onde $\widetilde{Q}$ é a densidade de corrente constante no estado estacionário no espaço $(x, K y, z)$ e pode ser relacionada com a corrente $I$ injetada em $\left(x_{S}, z_{S}\right)$ pela equação:

$$
\tilde{Q}=\frac{I}{2 \Delta A}
$$

sendo $\Delta A$ uma área representativa no plano $x z$ sobre o ponto de injeção de carga $\left(x_{\mathrm{S}}, z_{\mathrm{S}}\right)$.

O objetivo da modelagem ER 2D é obter as soluções numéricas da equação (41) sujeita as seguintes condições de contorno: o potencial elétrico $\phi(x, y, z)$ e a componente normal $(\bar{J} \equiv \partial \phi / \partial \eta)$ devem ser contínuos através de cada fronteira da distribuição de condutividade $\sigma(x, z)$. 
A solução de $\tilde{\phi}(x, K y, z)$ é obtida derivando a equação de diferença (41), pela discretização adequada do espaço $(x, K y, z)$ sobre o qual o problema deve ser resolvido.

A forma generalizada da equação (41) pode ser expressa como:

$$
\begin{gathered}
-\frac{\partial}{\partial x}\left(P(x, z) \frac{\partial \tilde{\phi}}{\partial x}(x, K y, z)\right)-\frac{\partial}{\partial z}\left(P(x, z) \frac{\partial \tilde{\phi}}{\partial z}(x, K y, z)\right) \\
+\sigma(x, z) \tilde{\phi}(x, K y, z)=f(x, z) ;(x, z) \in R
\end{gathered}
$$

onde o conjunto $R$ é considerado fechado, conectado e possui um interior não vazio.

O semi-espaço inferior com distribuição de condutividade arbitrária é definido de tal forma que o conjunto $R$ apresenta limites infinitamente distantes nas direções $x$ e $z$. A malha é retangular e os nós possuem espaçamento aleatório e irregular. Os nós na direção $x$ são indexados por $i=1,2,3 \ldots, N$, e os nós na direção $z$ por $j=1,2,3 \ldots, M$. As extremidades esquerda e direita são representadas pelas linhas $i=1$ e $i=N$, respectivamente, e a extremidade inferior pela linha $j=M$.

O potencial primário, devido a uma fonte pontual, e os potenciais secundários, devido as heterogeneidades de condutividade diminuem inversamente com a distância. Dessa forma, aumentando-se gradualmente o tamanho dos elementos, estabelecendo um número suficientemente grande para $M$ e $N$ e utilizando as condições de fronteira apropriadas, as extremidades infinitas podem ser aproximadas por um número finito de $M$ e $N$.

Aplica-se a equação (41) para todos os nós $(i, j)$ para representar uma aproximação através de uma área $\Delta A$, representada pela área cinza na Figura 16. Para uma fonte pontual no interior da malha obtém-se:

$$
\Delta A=\frac{\left(\Delta x_{i}+\Delta x_{i-1}\right) \cdot\left(\Delta z_{i}+\Delta z_{i-1}\right)}{4}
$$

e na superfície $(z \rightarrow 0)$

$$
\Delta A=\frac{\left(\Delta x_{i}+\Delta x_{i-1}\right) \cdot \Delta z_{i}}{4}
$$




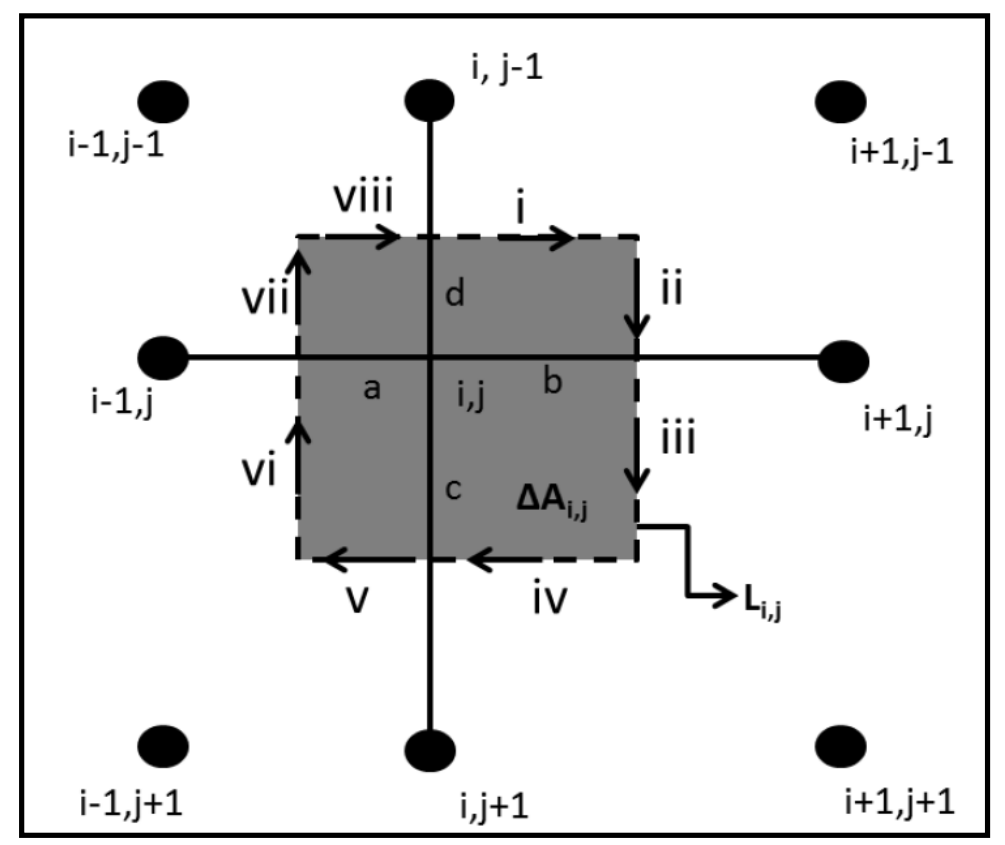

Figura 16 - Elemento de área discretizada $\Delta A_{i, j}$ (Bortolozo, 2016).

Na superfície $(z=0)$ o potencial é zero. As extremidades esquerda, direita e inferior são dadas pela ampliação da malha a distâncias suficientemente grandes, de modo que a distribuição do potencial total nessas extremidades se aproxima de um comportamento assintótico. Assim, os potenciais nas extremidades podem ser definidos a partir do potencial de um semi-espaço homogêneo e as heterogeneidades são consideradas como perturbações dessa distribuição homogênea.

A derivação das equações de diferenças finitas é realizada através da discretização por pontos. A distribuição de condutividade $\sigma(x, z)$ é discretizada em cada nó por $\sigma i, j$ e na solução numérica obtém-se um valor de potencial $\tilde{\phi}_{i, j}$ para cada nó. Assim, a equação de diferença para qualquer nó interior $(i, j)$ da malha é dada por:

$$
C_{L}^{i j} \tilde{\phi}_{i-1, j}+C_{R}^{i j} \tilde{\phi}_{i+1, j}+C_{T}^{i j} \tilde{\phi}_{i, j-1}+C_{B}^{i j} \tilde{\phi}_{i, j+1}+C_{P}^{i j} \tilde{\phi}_{i, j}=2 \tilde{Q} \delta\left(x_{s}\right) \delta\left(z_{s}\right)
$$

onde $C_{L}^{i j}$ é o coeficiente de acoplamento entre os nós $(i, j)$ e $(i-1, j), C_{R}^{i j}$ entre $(i, j)$ e $(i+1$, $j), C_{T}^{i j}$ entre $(i, j)$ e $(i, j-1), C_{B}^{i j}$ entre $(i, j)$ e $(i, j+1)$ e $C_{P}^{i j}$ o auto acoplamento do nó $(i, j)$.

A equação de diferença (46) mostra que a solução de $\tilde{\phi}$ no nó $(i, j)$ depende apenas dos valores de $\tilde{\phi}$ nos nós vizinhos $(i-1, j),(i+1, j),(i, j-1)$ e $(i, j+1)$. Os coeficientes de acoplamento $(C)$ são conhecidos em todos os nós do conjunto $R$, pois são funções dos valores de condutividade $\sigma i, j$ e da geometria da malha. A equação (46) é válida para qualquer 
distribuição de $\sigma i, j(0<\sigma<\infty)$ e, portanto, as condições de fronteira necessárias para a continuidade de $\tilde{\phi}$ e $\sigma \partial \tilde{\phi} / \partial \eta$ são satisfeitas para qualquer ponto no interior da malha. No entanto, as equações de diferença são um pouco diferentes para os nós localizados na superfície e nas extremidades esquerda, direita e inferior, conforme demonstrado a seguir:

1. Nós na superfície $(\boldsymbol{z}=\mathbf{0})$ : para todos $(i, j) \operatorname{com} i=2,3, \ldots, N-1, j=1$, temos:

$$
C_{L}^{i j} \tilde{\phi}_{i-1, j}+C_{R}^{i j} \tilde{\phi}_{i+1, j}+C_{B}^{i j} \tilde{\phi}_{i, j+1}+C_{P}^{i j} \tilde{\phi}_{i, j}=2 \widetilde{Q} \delta\left(x_{s}\right) \delta\left(z_{s}\right)
$$

2. Nós no topo esquerdo e direito $(\boldsymbol{z}=\mathbf{0})$ : para o nó superior esquerdo $(1,1)$ :

$$
C_{R}^{i j} \tilde{\phi}_{i+1, j}+C_{B}^{i j} \tilde{\phi}_{i, j+1}+C_{P}^{i j} \tilde{\phi}_{i, j}=2 \tilde{Q} \delta\left(x_{S}\right) \delta\left(z_{S}\right)
$$

e para o nó superior direito $(N, 1)$ :

$$
C_{L}^{i j} \tilde{\phi}_{i-1, j}+C_{B}^{i j} \tilde{\phi}_{i, j+1}+C_{P}^{i j} \tilde{\phi}_{i, j}=2 \tilde{Q} \delta\left(x_{s}\right) \delta\left(z_{s}\right)
$$

3. Nós na extremidade inferior $(\mathbf{z}=\mathbf{M})$ : para todos $(i, M)$, com $i=2,3, \ldots, N-1$ :

$$
C_{L}^{i j} \tilde{\phi}_{i-1, j}+C_{R}^{i j} \tilde{\phi}_{i+1, j}+C_{T}^{i j} \tilde{\phi}_{i, j-1}+C_{P}^{i j} \tilde{\phi}_{i, j}=2 \tilde{Q} \delta\left(x_{s}\right) \delta\left(z_{s}\right)
$$

4. Nós nos cantos inferiores esquerdo e direito: para o nó $(1, M)$ :

$$
C_{R}^{i j} \tilde{\phi}_{i+1, j}+C_{T}^{i j} \tilde{\phi}_{i, j-1}+C_{P}^{i j} \tilde{\phi}_{i, j}=2 \tilde{Q} \delta\left(x_{s}\right) \delta\left(z_{s}\right)
$$

e para o nó $(N, M)$ :

$$
C_{L}^{i j} \tilde{\phi}_{i-1, j}+C_{T}^{i j} \tilde{\phi}_{i, j-1}+C_{P}^{i j} \tilde{\phi}_{i, j}=2 \tilde{Q} \delta\left(x_{s}\right) \delta\left(z_{S}\right)
$$

5. Nós na lateral esquerda: para todos $(1, j) \operatorname{com} j=2,3, \ldots, M-1$ :

$$
C_{R}^{i j} \tilde{\phi}_{i+1, j}+C_{T}^{i j} \tilde{\phi}_{i, j-1}+C_{B}^{i j} \tilde{\phi}_{i, j+1}+C_{P}^{i j} \tilde{\phi}_{i, j}=2 \tilde{Q} \delta\left(x_{S}\right) \delta\left(z_{S}\right)
$$


6. Nós na lateral direita: para todos $(N, j) \operatorname{com} j=2,3, \ldots, M-1$ :

$$
C_{L}^{i j} \tilde{\phi}_{i-1, j}+C_{T}^{i j} \tilde{\phi}_{i, j-1}+C_{B}^{i j} \tilde{\phi}_{i, j+1}+C_{P}^{i j} \tilde{\phi}_{i, j}=2 \tilde{Q} \delta\left(x_{s}\right) \delta\left(z_{S}\right)
$$

As equações de diferenças para todos os nós podem ser resolvidas simultaneamente na forma matricial. A matriz $M N x M N$ é chamada de matriz resistência ou capacitância $(C)$. A seguir é apresentado um exemplo de como a matriz resistência é montada.

A Figura 17 mostra um exemplo de uma malha com 16 nós, com quatro linhas e quatro colunas. Os nós são numerados de $k=1,2, \ldots, 16$ a partir do topo esquerdo até o canto inferior direito.

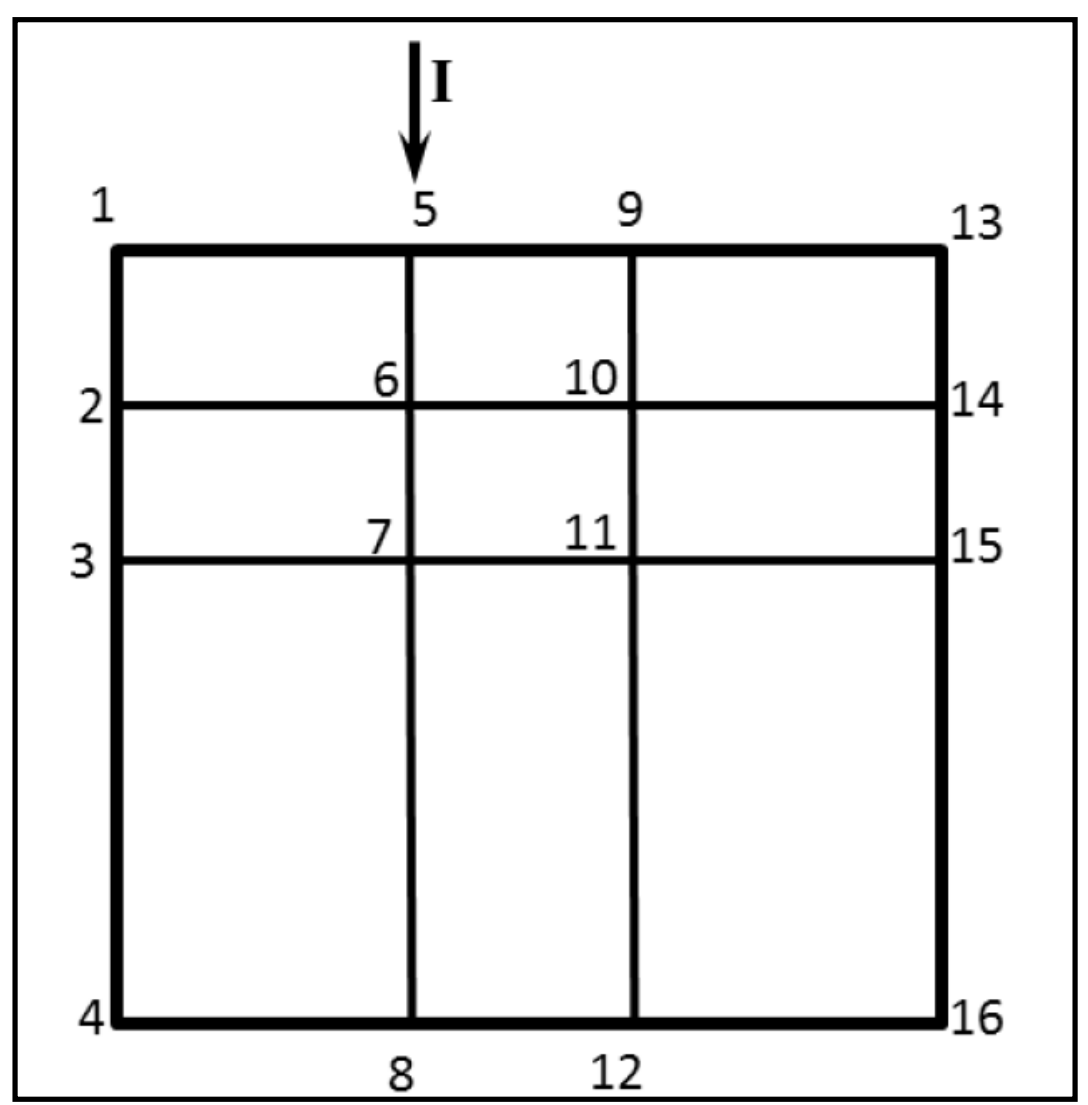

Figura 17 - Exemplo de numeração de nós para uma malha retangular com 16 nós (Bortolozo, 2016).

Na matriz $C$ cada nó $(i, j)$ é representado pela linha $k$ equivalente ao seu número e as colunas possuem valores diferentes de zero nas posições dos nós vizinhos ao nó $k$. Dessa forma a matriz $C$ para os 16 nós do exemplo é dada por: 


\begin{tabular}{|c|c|c|c|c|c|c|c|c|c|c|c|c|c|c|c|}
\hline$\left\lceil C_{P}(1)\right.$ & $C_{B}(1)$ & 0 & 0 & $C_{R}(1)$ & 0 & 0 & 0 & 0 & 0 & 0 & 0 & 0 & 0 & 0 & 0 \\
\hline$C_{T}(2)$ & $C_{P}(2)$ & $C_{B}(2)$ & 0 & 0 & $C_{R}(2)$ & 0 & 0 & 0 & 0 & 0 & 0 & 0 & 0 & 0 & 0 \\
\hline 0 & $C_{T}(3)$ & $C_{P}(3)$ & $C_{B}(3)$ & 0 & 0 & $C_{R}(3)$ & 0 & 0 & 0 & 0 & 0 & 0 & 0 & 0 & 0 \\
\hline 0 & 0 & $C_{T}(4)$ & $C_{P}(3)$ & 0 & 0 & 0 & $C_{R}(4)$ & 0 & 0 & 0 & 0 & 0 & 0 & 0 & 0 \\
\hline$C_{L}(5)$ & 0 & 0 & 0 & $C_{P}(5)$ & $C_{B}(5)$ & 0 & 0 & $C_{R}(5)$ & 0 & 0 & 0 & 0 & 0 & 0 & 0 \\
\hline 0 & $C_{L}(6)$ & 0 & 0 & $C_{T}(6)$ & $C_{P}(6)$ & $C_{B}(6)$ & 0 & 0 & $C_{R}(6)$ & 0 & 0 & 0 & 0 & 0 & 0 \\
\hline 0 & 0 & $C_{L}(7)$ & 0 & 0 & $C_{T}(7)$ & $C_{P}(7)$ & $C_{B}(7)$ & 0 & 0 & $C_{R}(7)$ & 0 & 0 & 0 & 0 & 0 \\
\hline 0 & 0 & 0 & $C_{L}(8)$ & 0 & 0 & $C_{T}(8)$ & $C_{P}(8)$ & 0 & 0 & 0 & $C_{R}(8)$ & 0 & 0 & 0 & 0 \\
\hline 0 & 0 & 0 & 0 & $C_{L}(9)$ & 0 & 0 & 0 & $C_{P}(9)$ & $C_{B}(9)$ & 0 & 0 & $C_{R}(9)$ & 0 & 0 & 0 \\
\hline 0 & 0 & 0 & 0 & 0 & $C_{L}(10)$ & 0 & 0 & $C_{T}(10)$ & $C_{P}(10)$ & $C_{B}(10)$ & 0 & 0 & $C_{R}(10)$ & 0 & 0 \\
\hline 0 & 0 & 0 & 0 & 0 & 0 & $C_{L}(11)$ & 0 & 0 & $C_{T}(11)$ & $C_{P}(11)$ & $C_{B}(11)$ & 0 & 0 & $C_{R}(11)$ & 0 \\
\hline 0 & 0 & 0 & 0 & 0 & 0 & 0 & $C_{L}(12)$ & 0 & 0 & $C_{T}(12)$ & $C_{P}(12)$ & 0 & 0 & 0 & $C_{R}(12)$ \\
\hline 0 & 0 & 0 & 0 & 0 & 0 & 0 & 0 & $C_{L}(13)$ & 0 & 0 & 0 & $C_{P}(13)$ & $C_{B}(13)$ & 0 & 0 \\
\hline 0 & 0 & 0 & 0 & 0 & 0 & 0 & 0 & 0 & $C_{L}(14)$ & 0 & 0 & $C_{T}(14)$ & $C_{P}(14)$ & $C_{B}(14)$ & 0 \\
\hline 0 & 0 & 0 & 0 & 0 & 0 & 0 & 0 & 0 & 0 & $C_{L}(15)$ & 0 & 0 & $C_{T}(15)$ & $C_{P}(15)$ & $C_{B}(15)$ \\
\hline 0 & 0 & 0 & 0 & 0 & 0 & 0 & 0 & 0 & 0 & 0 & $C_{L}(16)$ & 0 & 0 & $C_{T}(16)$ & $C_{P}(16)$ \\
\hline
\end{tabular}

A equação (55) pode ser escrita simbolicamente como:

$$
C \tilde{\phi}=S
$$

Como o objetivo é obter o potencial $\tilde{\phi}$, portanto o sistema linear a ser resolvido é dado por:

$$
\tilde{\phi}=C^{-1} S
$$

Dado que a matriz $C$ depende somente da geometria e da condutividade da malha, ela se mantém inalterada para qualquer posição de fonte de corrente. Portanto, a inversão da matriz $C$ possibilita a solução para os conjuntos de $\tilde{\phi}$ para as posições de origem que formam o vetor $S$.

O potencial elétrico $\phi(x, \mathrm{~K} y, z)$ é então obtido através da transformada inversa do potencial transformado $\tilde{\phi}(x, K y, z)$, dado por: 


$$
\phi(x, K y, z)=\frac{1}{\pi} \int_{0}^{\infty} \tilde{\phi}(x, K y, z) d \lambda
$$

A integral (58) é resolvida de forma numérica, sendo que o valor de $\lambda$ é obtido empiricamente por tentativa e erro.

A partir da diferença de potencial elétrico para uma configuração genérica de eletrodos como na Figura 7, a resistividade aparente $\left(\rho_{a}\right)$ é calculada pela equação (10).

\subsubsection{Modelagem TDEM 2D por Diferenças Finitas}

A modelagem TDEM 2D foi feita com base no artigo de Oristaglio e Hohmann (1984), que utilizaram o método das diferenças finitas para simular sondagens TDEM. Os autores assumiram um loop retangular aproximado por dois fios infinitos perpendiculares ao eixo y (strike geoelétrico) e utilizaram o método de diferenças finitas explícitas originalmente proposto por DuFort e Frankel (1953). A discretização do meio, representada na Figura 18, é da mesma forma que na modelagem ER 2D por diferenças finitas. A condutividade elétrica $\sigma(x, z)$ é constante dentro de cada elemento, mas pode variar entre os elementos no plano ( $x$, $z)$. A permeabilidade magnética $(\mu)$ e a permissividade dielétrica $(\varepsilon)$ são constantes nos seus valores no vácuo: $\mu=4 \pi \times 10^{-7} \mathrm{H} / \mathrm{m}$ e $\varepsilon=8.854 \times 10^{-12} \mathrm{~F} / \mathrm{m}$.

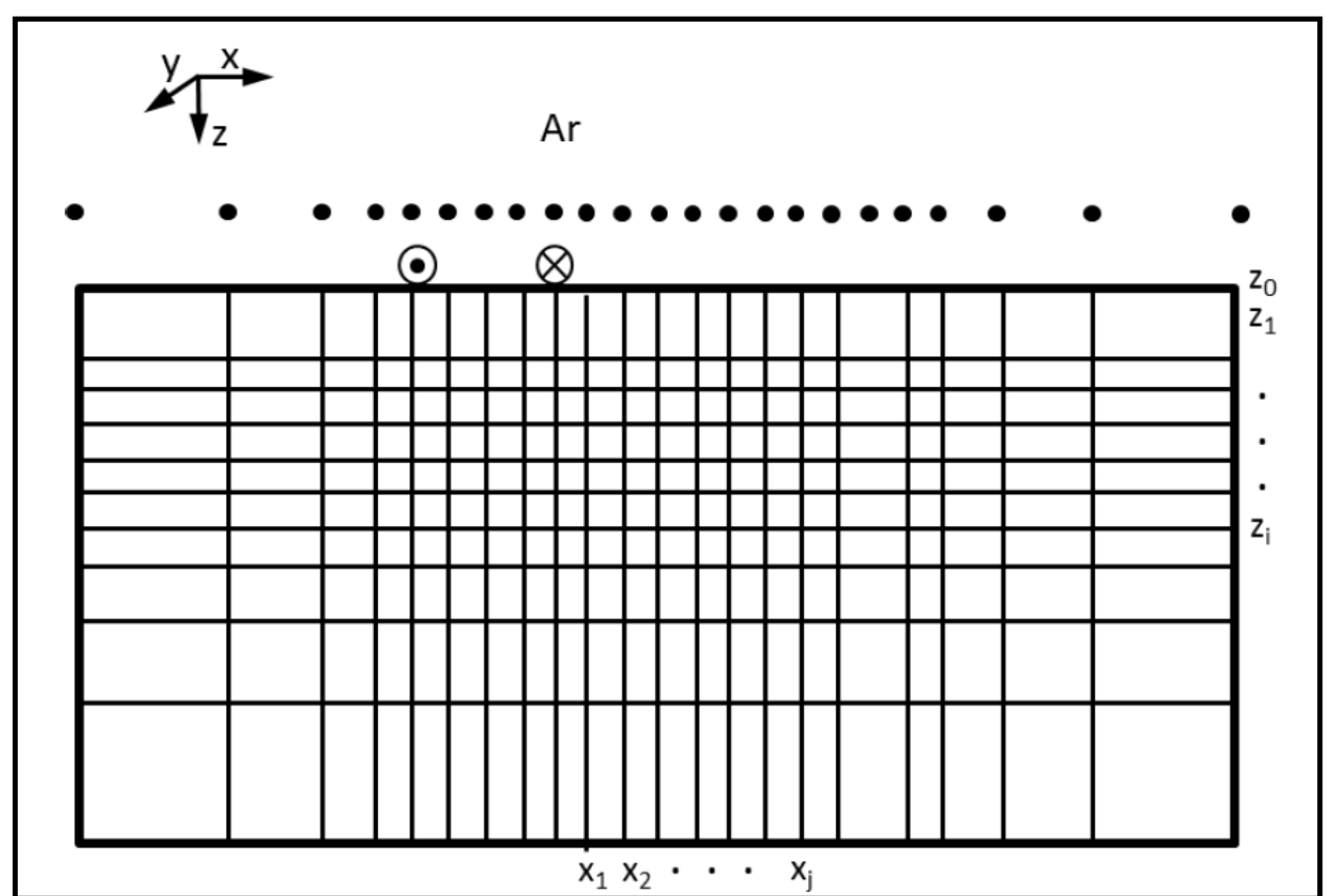

Figura 18 - Discretização TDEM 2D por diferenças finitas. Os pontos pretos no ar são necessários para a condição de fronteira na interface Terra-Ar (Bortolozo, 2016). 
As equações de Maxwell para os campos elétricos e magnéticos no modo transiente 2D são dadas por:

$$
\begin{gathered}
\boldsymbol{E}(x, z, t)=E_{y} \hat{y} \\
\boldsymbol{H}(x, z, t)=H_{x} \hat{x}+H_{z} \hat{z}
\end{gathered}
$$

As equações (59) e (60) podem ser reduzidas a uma equação escalar para o campo elétrico na direção do strike geoelétrico, tomando $E y=E$ :

$$
\partial_{x x} E+\partial_{z z} E-\mu \sigma \partial_{t} E-\mu \varepsilon \partial_{t t} E=\mu \partial_{t} J_{S}
$$

onde $J S$ é a densidade da corrente na direção $y$. A equação (61) é uma equação de onda amortecida.

Em materiais geológicos a condutividade elétrica $(\sigma)$ varia da ordem de $10^{-4}$ a $10^{3}$, enquanto que a permissividade elétrica $(\varepsilon)$, considerada como a do vácuo, tem a ordem de $10^{-12}$. Portanto, o quarto termo da equação (61) pode ser desprezado e $E$ obedece a seguinte equação de difusão 2D:

$$
\partial_{x x} E+\partial_{z z} E-\mu \sigma \partial_{t} E=\mu \partial_{t} J_{S}
$$

O limite de difusão começa em tempos muito pequenos para a maioria dos modelos geofísicos. Assim, para distâncias pequenas e intervalos de tempo curtos, a equação de difusão se aproxima assintoticamente para a seguinte condição que é sempre satisfeita em sondagens TDEM:

$$
\left(t^{2}-R^{2} / c^{2}\right)^{1 / 2} \gg \frac{2 \varepsilon}{\sigma}
$$

onde $R=\left(x^{2}+z^{2}\right)^{1 / 2}$ é a distância da origem e $c=(\mu \varepsilon)^{-1 / 2}$ a velocidade da luz no vácuo.

$\mathrm{O}$ intervalo de tempo para a difusão $2 \mathrm{D}$ com espaçamento $\Delta$, é dado por:

$$
\Delta t_{1}=\frac{\mu \sigma \Delta^{2}}{4}
$$


sendo $\Delta t_{1}$ o passo de tempo máximo utilizado na solução explícita da equação de difusão.

A aproximação por diferenças finitas para os termos espaciais da equação de difusão, omitindo-se os termos fonte, é dada por:

$$
\mu \sigma \partial_{t} E=\partial_{x x} E+\partial_{z z} E
$$

A Figura 19 representa um ponto $E_{i, j}$ na malha cercado por seus vizinhos $E_{i+1, j}$, $E_{i-1, j}, E_{i, j+1}, E_{i, j-1}$. Integrando a equação (65) sobre o retângulo $A B C D$ formado pela união dos pontos médios dos quatro retângulos, obtém-se:

$$
\begin{gathered}
\iint_{A B C D} d x d z \mu \sigma \partial_{t} E=\iint_{A B C D} d x d z\left(\partial_{x x} E+\partial_{z z} E\right) \\
=\int_{B C} d x \partial_{z} E-\int_{A D} d x \partial_{z} E+\int_{D C} d z \partial_{x} E-\int_{A B} d z \partial_{x} E
\end{gathered}
$$

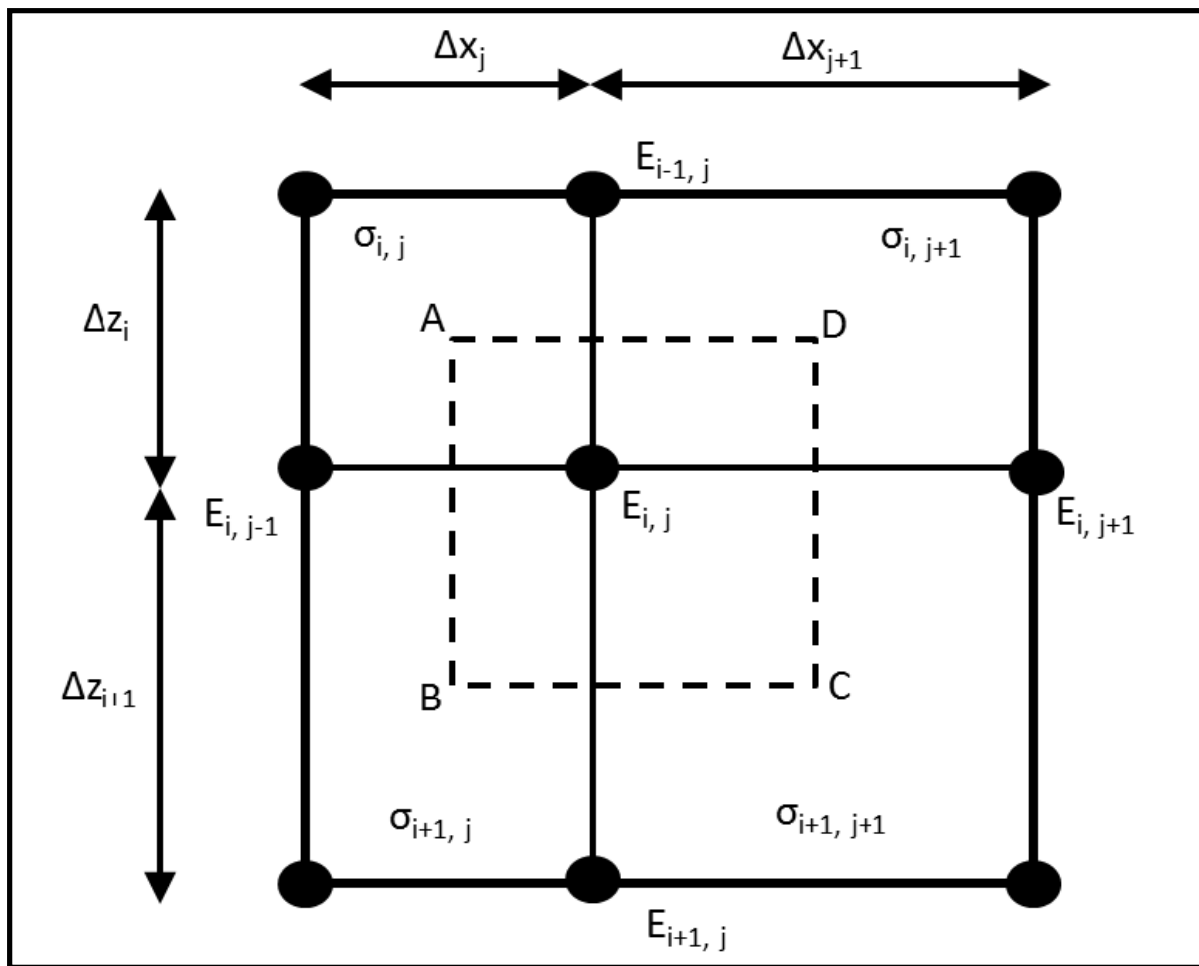

Figura 19 - Ponto $E_{i, j}$ na malha de diferenças finitas, cercado por seus vizinhos e elementos de condutividade constante. ABCD é o retângulo formado pela união dos pontos centrais dos quadrados ao redor de $E_{i, j}$ (Bortolozo, 2016).

Resolvendo a integral (66), obtém-se: 


$$
\begin{aligned}
\mu \bar{\sigma}_{i, j} \partial_{t} E_{i, j}= & \frac{1}{\Delta z_{i} \Delta z_{i+1}}\left(\frac{2 \Delta z_{i+1}}{\Delta z_{i}+\Delta z_{i+1}} E_{i-1, j}+\frac{2 \Delta z_{i}}{\Delta z_{i}+\Delta z_{i+1}} E_{i+1, j}-2 E_{i, j}\right) \\
& +\frac{1}{\Delta x_{j} \Delta x_{j+1}}\left(\frac{2 \Delta x_{j+1}}{\Delta x_{j}+\Delta x_{j+1}} E_{i, j-1}+\frac{2 \Delta x_{j}}{\Delta x_{j}+\Delta x_{j+1}} E_{i, j+1}-2 E_{i, j}\right)
\end{aligned}
$$

onde $\bar{\sigma}_{i, j}$ é a média ponderada por área das condutividades circundantes à $E_{i, j}$ dada por:

$$
\bar{\sigma}_{i, j}=\frac{\sigma_{i, j} \Delta z_{i} \Delta x_{j}+\sigma_{i+1, j} \Delta z_{i+1} \Delta x_{j}+\sigma_{i, j+1} \Delta z_{i} \Delta x_{j+1}+\sigma_{i+1, j+1} \Delta z_{i+1} \Delta x_{j+1}}{\left(\Delta z_{i}+\Delta z_{i+1}\right)\left(\Delta x_{j}+\Delta x_{j+1}\right)}
$$

e o lado direito da equação (67) é a diferença aproximada em 5 pontos do Laplaciano em uma malha irregular.

Os tempos de difusão variam de acordo com a distribuição de condutividade elétrica no interior da malha. Para estimar a constante de decaimento, pode-se assumir um elemento de lados $L_{x}$ e $L_{z}$ e condutividade $\sigma$, cercado por um condutor perfeito. A maior constante de tempo do elemento retangular, que é da ordem de milissegundos, é dada por:

$$
\tau_{2 D}=\frac{\sigma \mu \bar{L}^{2}}{\pi^{2}}
$$

onde

$$
\frac{1}{\bar{L}^{2}}=\frac{1}{L_{x}^{2}}+\frac{1}{L_{z}^{2}}
$$

De acordo com Oristaglio e Hohmann (1984), o método mais adequado para a solução do problema de modelagem TDEM 2D é o DuFort-Frankel, que é estável e explícito. Adiciona-se um termo hiperbólico à equação de difusão para obter-se a estabilidade e considera-se inicialmente um modelo simples, onde os espaçamentos são iguais $(\Delta x=\Delta z=$ $\Delta)$. O comportamento difusivo irá dominar quando:

$$
t \gg \frac{4 \Delta t^{2}}{\mu \sigma \Delta^{2}}
$$


Assim, resultados cada vez mais precisos são obtidos para a equação de difusão a medida que $t$ se torna maior em relação ao lado direito da equação (71). Transformando a inequação (71) em uma equação e resolvendo para $\Delta t$, obtém-se o resultado para o máximo passo no tempo para o método DuFort-Frankel:

$$
\Delta t_{D F F}=(\mu \sigma t)^{1 / 2} \frac{\Delta}{2}
$$

O algoritmo desenvolvido utiliza como passo máximo o menor valor entre $\Delta t_{D F F} \mathrm{e}$ $\Delta t_{1}$, o que proporciona uma maior precisão mesmo com maior custo computacional. Além disso, para obter-se resultados melhores, o tempo inicial é dado por $t_{0}=\Delta t_{D F F}$.

A generalização do método DuFort-Frankel para uma malha irregular é dada por:

$$
\begin{gathered}
E_{i, j}^{n+1}=\frac{1-4 \bar{r}_{i, j}}{1+4 \bar{r}_{i, j}} E_{i, j}^{n-1}+\frac{2 r_{i, j}^{z}}{1+4 \bar{r}_{i, j}}\left(\frac{\Delta z_{i}}{\overline{\Delta z_{l}}} E_{i+1, j}^{n}+\frac{\Delta z_{i+1}}{\overline{\Delta z_{l}}} E_{i-1, j}^{n}\right) \\
+\frac{2 r_{i, j}^{x}}{1+4 \bar{r}_{i, j}}\left(\frac{\Delta x_{j}}{\overline{\Delta x_{J}}} E_{i, j+1}^{n}+\frac{\Delta x_{j+1}}{\overline{\Delta x_{J}}} E_{i, j-1}^{n}\right)
\end{gathered}
$$

onde $\overline{\Delta z_{l}}$ e $\overline{\Delta x_{J}}$ são os espaçamentos médios nas direções $x$ e $z$ e $\bar{r}_{i, j}$ é a média das razões de malha dada por:

$$
\bar{r}_{i, j}=\frac{r_{i, j}^{x}+r_{i, j}^{Z}}{2}
$$

No modo transiente 2D o campo elétrico e seu gradiente são contínuos em todas as fronteiras, portanto, as únicas condições de contorno que precisam de tratamento especial são as quatro extremidades da malha.

A condição de radiação no ar, onde o campo elétrico satisfaz a equação de Laplace sob a aproximação quase estática, é dada por:

$$
\partial_{x x} E+\partial_{z z} E=0
$$

Assim, o campo elétrico no ar pode ser calculado a partir do seu valor na interface terra-ar $E(\mathrm{x}, \mathrm{z}=0, \mathrm{t})$ por uma continuação para cima. No primeiro passo de tempo, o campo 
elétrico em $z=0$ pode ser calculado pela equação (75) a uma distância $\Delta z$ acima da interface, representado pelos pontos pretos na Figura 18. Após obter este valor, a equação de diferença (75) é utilizada para calcular o campo elétrico para o próximo valor de tempo. Esse ciclo pode ser repetido para qualquer número de passos de tempo.

Uma vez que as fronteiras laterais e inferior da malha não têm uma implementação numérica simples, a solução é estender essas fronteiras para que fiquem longe das heterogeneidades. Assim, aumenta-se gradativamente o tamanho dos elementos em direção as extremidades. Além disso, são definidos valores iguais a solução de um semi-espaço homogêneo. Dessa maneira se reduz a instabilidade numérica e garante-se que o aumento do tempo de cálculo, devido ao aumento do número de elementos, seja compensado com o aumento de precisão.

Considerando um semi-espaço homogêneo e uma fonte formada por um fio positivo, a partir da lei de Faraday na forma diferencial, obtém-se as seguintes expressões para as derivadas temporais dos campos magnéticos verticais e horizontais na superfície $(z=0)$ :

$$
\begin{gathered}
\frac{\partial B_{z}}{\partial t}=-\frac{\mu I}{2 \pi x} \frac{4}{\mu \sigma x^{2}}\left[e^{-x^{2} / T}\left(1+\frac{x^{2}}{T}\right)-1\right]=\frac{\mu I}{2 \pi x} \frac{1}{t} \sum_{n=1}^{\infty} \frac{(-1)^{n+1} n}{(n+1) !}\left(\frac{x^{2}}{T}\right)^{n} \\
\frac{\partial B_{x}}{\partial t}=\frac{\mu I}{2 \pi x} \frac{2}{\sqrt{\pi}} \frac{1}{t}\left[\frac{T^{1 / 2}}{x}-F\left(x T^{1 / 2}\right)\left(1+\frac{T}{x^{2}}\right)\right] \\
=\frac{\mu I}{2 \pi x} \frac{2}{\sqrt{\pi}} \frac{1}{t} \sum_{n=0}^{\infty} \frac{(-1)^{n}(2 n+1)(n+1) !}{(2 n+3) !}\left(\frac{4 x^{2}}{T}\right)^{n+1 / 2}
\end{gathered}
$$

onde $T$ (unidade de $\mathrm{m}^{2}$ ) é uma variável de tempo normalizada dada por:

$$
T=\frac{4 t}{\mu \sigma}
$$

Aplicando-se ao campo elétrico o mesmo tipo de derivada espacial, obtém-se a variação temporal do campo magnético na direção $z(\partial B z / \partial t)$ para um meio heterogêneo. Com $\partial B z \partial t$ é possível obter a resistividade aparente $\left(\rho_{a}\right)$ para o TDEM 2D: 


$$
\rho_{a}=\frac{\mu^{2} L_{f}}{16 \pi t^{2} \partial B_{z} / \partial t}
$$

onde $L_{f}$ é a distância entre os fios do loop transmissor.

A equação (79) é válida somente para medidas realizadas no interior do loop transmissor, porque quando a bobina receptora é colocada do lado de fora do loop transmissor, obtêm-se valores negativos de resistividade aparente (Bortolozo et al., 2016).

Informações detalhadas sobre as derivações das equações de Maxwell para o método TDEM podem ser encontradas em Christiansen et al. (2006) e Bortolozo (2016), dentre outros.

\subsection{Algoritmo de Inversão}

Conforme demonstrado anteriormente, foi utilizada a modelagem 2D por diferenças finitas para o cálculo direto para os métodos ER e TDEM. Assim, ambas as formulações são as mais compatíveis possíveis na inversão conjunta, possuindo a mesma malha e o mesmo tipo de discretização.

Dessa forma, Bortolozo (2016) desenvolveu um conjunto de rotinas em MATLAB para modelagem e inversão individual e conjunta de dados de CE e CTDEM.

O algoritmo de inversão, que é o mesmo tanto para a inversão individual quanto para a inversão conjunta, foi desenvolvido a partir do método de Occam para modelos suavizados. Como referência foi utilizado o algoritmo desenvolvido por Sasaki (1989), que realizou uma inversão conjunta de dados de eletrorresistividade e magnetotelúrico. Esse algoritmo determina iterativamente as resistividades dos elementos retangulares que representam o modelo geoelétrico bidimensional (2D).

As derivadas parciais são calculadas para um meio homogêneo pelo método das diferenças finitas apenas antes do processo iterativo, formando o primeiro conjunto de derivadas do algoritmo na matriz Jacobiana. Utiliza-se então o método de Broyden (1965) para atualizar a matriz. Esse método fornece uma generalização do método de Newton unidimensional, substituindo a derivada da matriz. A matriz Jacobiana é determinada de forma iterativa com base na equação de aproximação da diferença finita dada por:

$$
J_{n} \Delta x_{n} \approx \Delta f_{n}
$$


onde $n$ é o índice de iteração. A estimativa atual da Jacobiana $J n-1$ é utilizada para se obter a matriz para a próxima iteração Jn da seguinte forma:

$$
J_{n}=J_{n-1}+\frac{\Delta f_{n}-J_{n-1} \Delta x_{n}}{\left\|\Delta x_{n}\right\|^{2}} \Delta x_{n}^{T}
$$

Uma restrição de suavidade é adicionada na inversão para remover valores de resistividade espúrios que podem ocorrer devido a discretização em elementos menores do que a resolução espacial dos dados. Assim, busca-se o modelo que melhor ajuste os dados com uma variação suave a cada iteração.

O erro de ajuste dos resultados é calculado por:

$$
\Phi=\frac{\sum_{k=1}^{n}\left\|\left(d_{O B S}(k)-h_{P}(k)\right)\right\|}{\sum_{k=1}^{n} d_{O B S}(k)} \times 100
$$

onde $d_{o B S}$ são os dados reais ou sintéticos, $h_{P}$ o operador do cálculo direto e $n$ o número total de dados. A equação (82) representa a porcentagem do erro total de ajuste pelo valor total do somatório dos dados. A desvantagem desse cálculo de erro é que se existem valores de resistividade aparente muito mais altos que outros, o valor do erro $\Phi$ é alto. Por isso, o erro de ajuste obtido pela equação (82) não deve ser interpretado como um valor normal de RMS, pois não considera os erros percentuais ponto a ponto.

A Figura 20 apresenta de forma simplificada o conjunto de soluções possíveis. O conjunto A representa as soluções possíveis para a inversão de um dado de CE e o B para a inversão de um dado de CTDEM. Como ambos os métodos geofísicos investigam a mesma propriedade física, a resistividade elétrica, existe um conjunto de soluções que satisfazem as duas bases de dados, representado pelo conjunto C. Essa é a principal vantagem da inversão conjunta, ela reduz o espaço de soluções possíveis, diminuindo as ambiguidades e permitindo que a estimativa dos parâmetros seja mais confiável. 


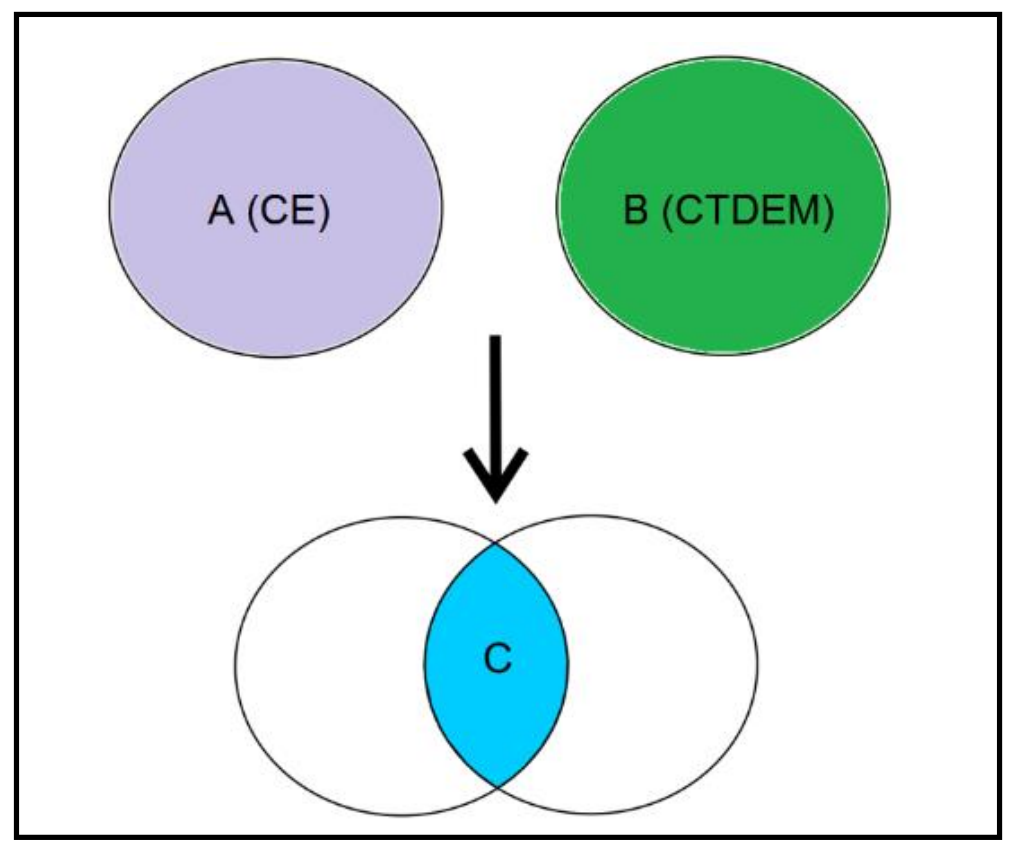

Figura 20 - Conjunto de soluções possíveis: A para a inversão de um dado de CE, B para a inversão de um dado de CTDEM e C para a inversão conjunta. 


\section{AQUISIÇÃO E TRATAMENTO DOS DADOS}

Neste capítulo são apresentados todos os parâmetros de aquisição de dados realizados na área de estudo, que incluem dados ER e TDEM em uma e duas dimensões. Porém, para as inversões foram utilizados apenas os dados 2D: caminhamento elétrico (CE) e caminhamento TDEM (CTDEM).

A Figura 21 apresenta um esquema geral dos arranjos de aquisição de dados ER e TDEM utilizados. As aquisições 1D e 2D são sobrepostas para que possam ser invertidas conjuntamente, ou seja, as aquisições SEV e TDEM loop central são sobrepostas e CE e CTDEM também sobrepostas.

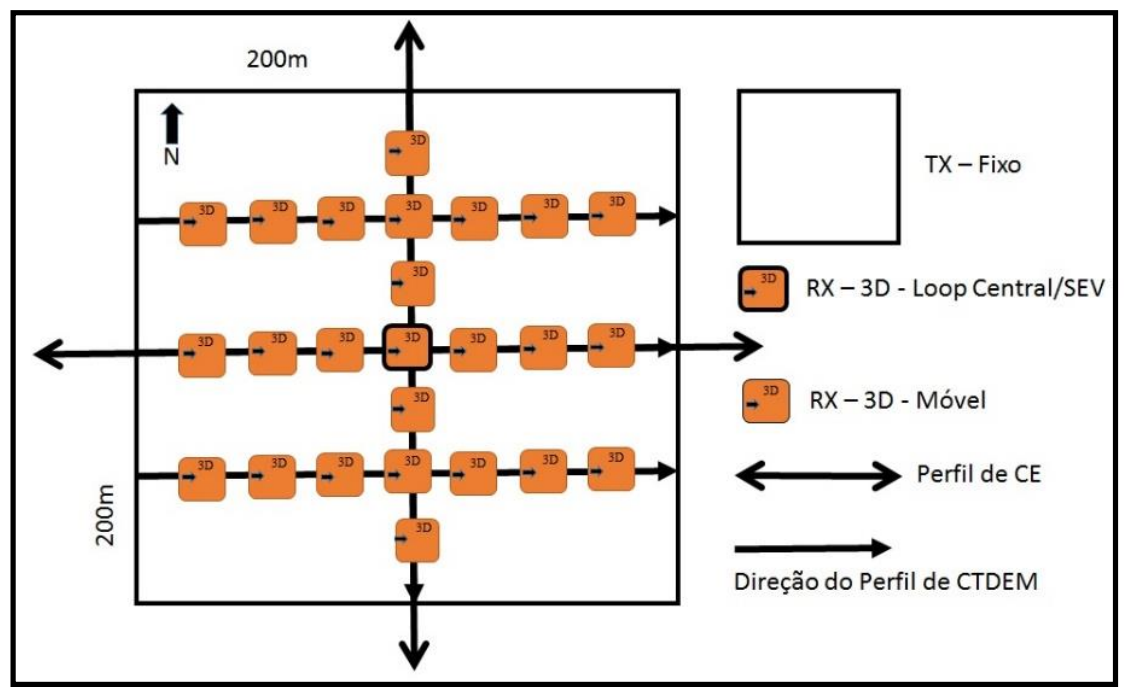

Figura 21 - Esquema geral dos arranjos de aquisição de dados ER e TDEM utilizados. Sondagens SEV e TDEM loop central sobrepostas. Quatro perfis de CTDEM e dois perfis de CE nas direções NS e EW.

As aquisições de dados ER foram feitas com as técnicas de Sondagem Elétrica Vertical (1D) e Caminhamento Elétrico (2D). As SEV's foram realizadas com o equipamento Syscal R2 da Iris Instruments (Figura 22a) e arranjo Schlumberger. Já os CE’s foram realizados com o equipamento Syscal PRO da Iris Instruments (Figura 22b), arranjos polodipolo e dipolo-dipolo.

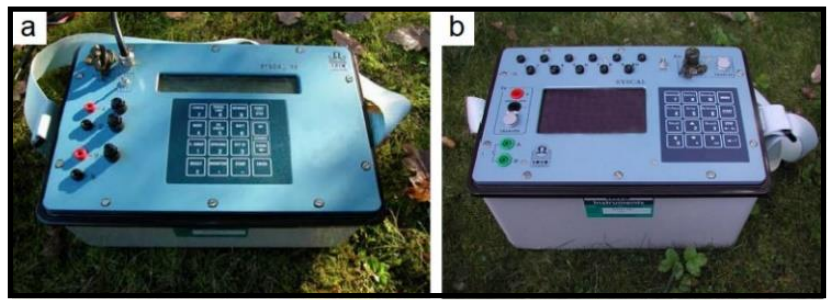

Figura 22 - Equipamentos utilizados na aquisição dos dados ER: a) Resistivímetro Syscal R2 para SEV e b) Resistivímetro Syscal PRO para CE, ambos da Iris Instruments. 
As aquisições de dados TDEM foram feitas com os arranjos loop central (1D) e CTDEM (2D) com o loop transmissor fixo e a bobina receptora móvel, sendo o loop transmissor com $200 \mathrm{~m}$ de lado. Cada CTDEM incluiu 7 sondagens, com $25 \mathrm{~m}$ de espaçamento entre as posições da bobina receptora, perfazendo $150 \mathrm{~m}$ de comprimento. Foram utilizados os seguintes equipamentos da Geonics (Figura 23): transmissor TEM57MK2, receptor PROTEM e bobina receptora 3D com área efetiva de $200 \mathrm{~m}^{2}$. A fonte de corrente elétrica utilizada foi um gerador Honda a gasolina com potência de $2 \mathrm{~kW}$ (Figura 23a), que forneceu uma corrente entre 17,5 A e 18,0 A. Para todas as medidas foram utilizadas 20 janelas (gates) de aquisição e frequências de $30 \mathrm{~Hz}, 7,5 \mathrm{~Hz}$ e $3 \mathrm{~Hz}$, sendo que quanto menor a frequência, maior o intervalo de tempo e, consequentemente, maior a profundidade de investigação. Foram medidas as 3 componentes do campo magnético secundário (vertical $z$ e horizontais $x$ e $y$ ). Para cada frequência as medidas são feitas durante um intervalo de integração, que foi de 30 ou 60 s, e estas medidas são empilhadas para melhorar a relação sinal/ruído.

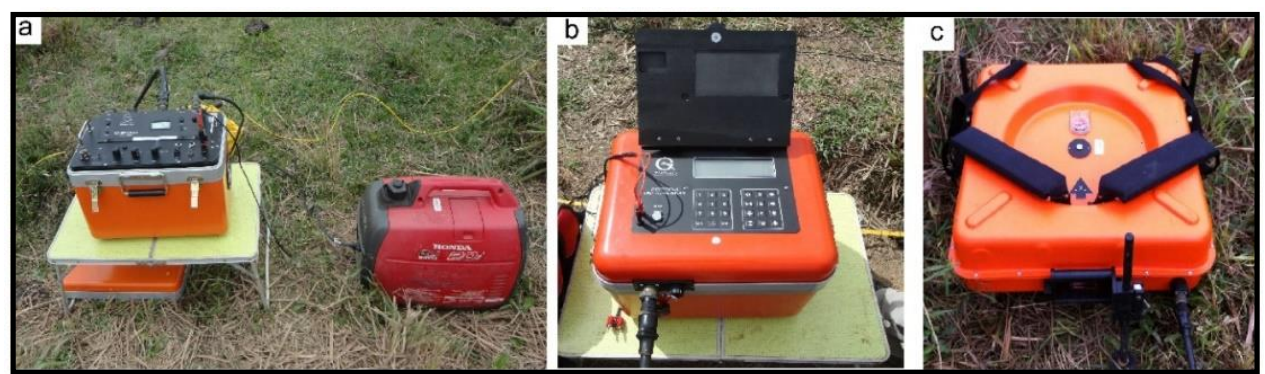

Figura 23 - Equipamentos utilizados na aquisição dos dados TDEM: a) Transmissor TDEM 57-MK2 e gerador; b) Receptor PROTEM e c) Bobina receptora 3D.

Foram realizadas 3 campanhas de aquisição de dados em 2016. Ao todo, foram adquiridas 3 SEV's, 4 CE's e 8 CTDEM's, totalizando 50 sondagens TDEM. A Figura 24 apresenta um mapa de localização de todas as aquisições realizadas.

A primeira campanha foi realizada entre os dias 25 e 29 de abril de 2016, quando foram realizados 4 CTDEM's (círculos pretos dentro do loop vermelho na Figura 24) e uma SEV no centro do loop vermelho com abertura máxima dos eletrodos de corrente $A B / 2$ de 275 m.

Na segunda campanha foram adquiridos 4 CE's entre os dias 1 e 4 de junho de 2016. O primeiro e o segundo CE's (pontos azuis na Figura 24) foram realizados com o arranjo polo-dipolo, ambos com $400 \mathrm{~m}$ de comprimento, sendo o início e o fim à $100 \mathrm{~m}$ do lado do loop vermelho, e espaçamento entre os eletrodos de $10 \mathrm{~m}$ e $20 \mathrm{~m}$, respectivamente. O terceiro 
$\mathrm{CE}$ (pontos verdes na Figura 24) foi feito com o arranjo polo-dipolo e espaçamento de $10 \mathrm{~m}$ entre os eletrodos, também com $400 \mathrm{~m}$ de extensão e início e fim à $100 \mathrm{~m}$ do lado do loop vermelho. O quarto e último $\mathrm{CE}$ (pontos amarelos na Figura 24) foi feito com o arranjo dipolo-dipolo, $20 \mathrm{~m}$ de espaçamento entre os eletrodos e percorreu a linha central dos dois loops totalizando $660 \mathrm{~m}$ de comprimento.

A terceira campanha foi realizada entre os dias 3 e 7 de setembro de 2016. Similar à primeira campanha, foram realizados 4 CTDEM's (círculos pretos dentro do loop azul na Figura 24) e 2 SEV's, uma no centro do loop azul e outra na interface entre os loops (seta branca na Figura 24), com abertura máxima dos eletrodos de corrente $A B / 2$ de $150 \mathrm{~m}$ e 200 $\mathrm{m}$, respectivamente. A Figura 25 apresenta duas fotos da terceira campanha.

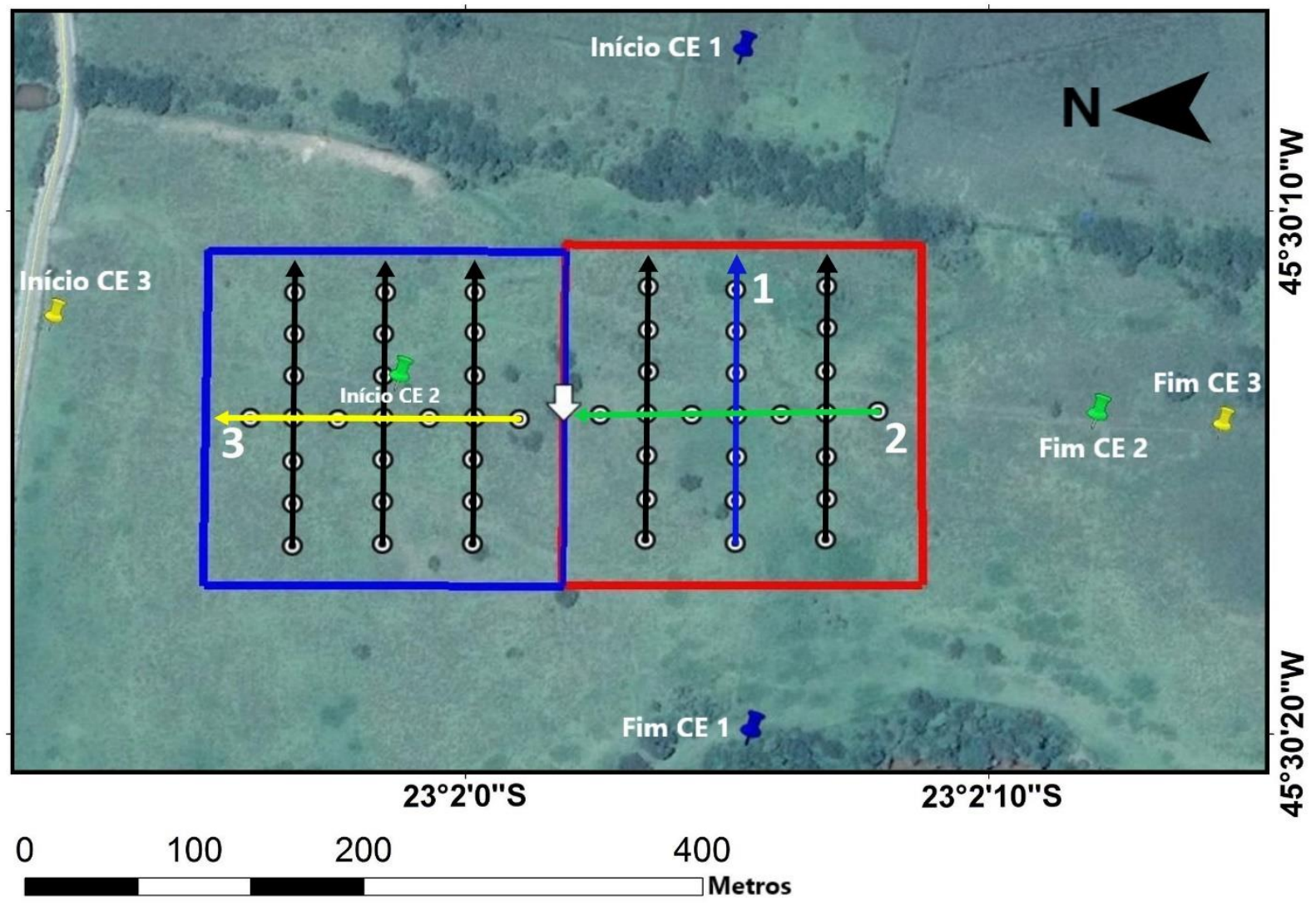

Figura 24 - Mapa de localização das aquisições de dados. Os quadrados azul e vermelho representam os loops transmissores e todos os círculos pretos dentro dos loops representam as sondagens TDEM. Os perfis de CE's são representados pelos pontos azuis (E-W), amarelos (N-S) e verdes (N-S) e as SEV's foram realizadas nas posições centrais dos dois loops e também na interface representada pela seta branca. Os perfis de CTDEM numerados de 1 a 3 foram utilizados nas Inversões Conjuntas 2D de CE e CTDEM. 


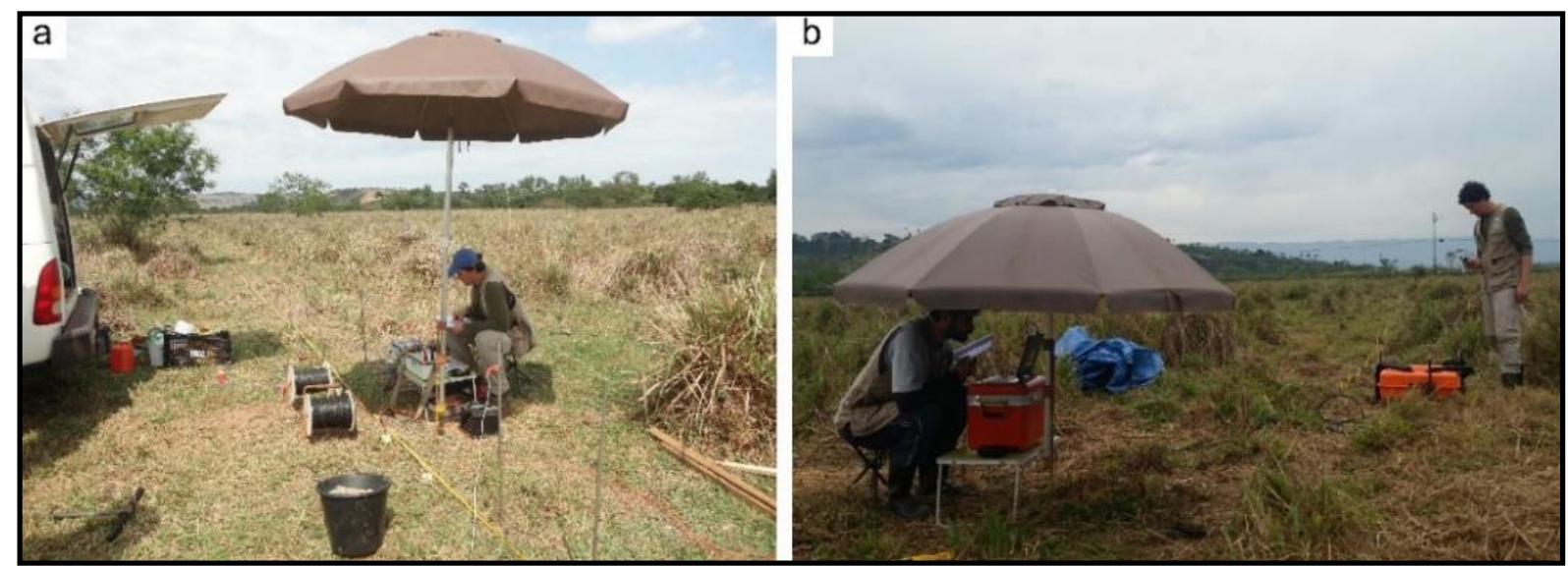

Figura 25 - Fotos da terceira campanha de aquisição de dados: a) Aquisição SEV e b) Aquisição TDEM.

Durante todas as aquisições de dados TDEM, nenhuma fonte de acoplamento influenciou as medidas, pois as redes de energia elétrica e cercas metálicas estavam a mais de 100 m distância, o que é considerada uma distância segura (Christiansen et al., 2006). Além disso, os dados foram adquiridos em um terreno praticamente plano, o que tem a vantagem de não exigir correção topográfica no processamento dos dados.

Os dados de CE precisam ser editados para remoção de possíveis pontos espúrios e, no caso dos dados adquiridos em Taubaté, não foram observados nenhum ponto espúrio. Já no caso dos dados de CTDEM, é necessário utilizar o software IX1D (Interpex) para remover os pontos espúrios e converter os dados para resistividade aparente em função do tempo. As mesmas edições devem ser aplicadas para as 7 sondagens do CTDEM, obtendo-se os mesmos intervalos de tempo e, consequentemente, o mesmo número de pontos. Embora foram medidas as 3 componentes do campo magnético secundário $(\mathrm{H} x, \mathrm{H} y$ e $\mathrm{Hz})$, utilizou-se apenas a componente vertical $(\mathrm{Hz})$ para as inversões.

As Figuras 26 e 27 apresentam um exemplo de uma curva de dados de resistividade aparente em função do tempo antes e depois do tratamento, respectivamente. O dado bruto possui 20 pontos para cada frequência, que correspondem as janelas (gates) de aquisição, totalizando 60 pontos. As curvas das 3 frequências são concatenadas e os pontos discrepantes que estão espalhados em relação à tendência da curva de resistividade aparente são eliminados. Os pontos removidos são normalmente associados a ruído ou perda de sinal para maiores tempos de aquisição, sendo o ruído ambiental assumido como $0,5 \mathrm{nV} / \mathrm{m}^{2}$ (Spies, 1989). A análise da tendência da curva de dados é fundamental para o processo de inversão. 


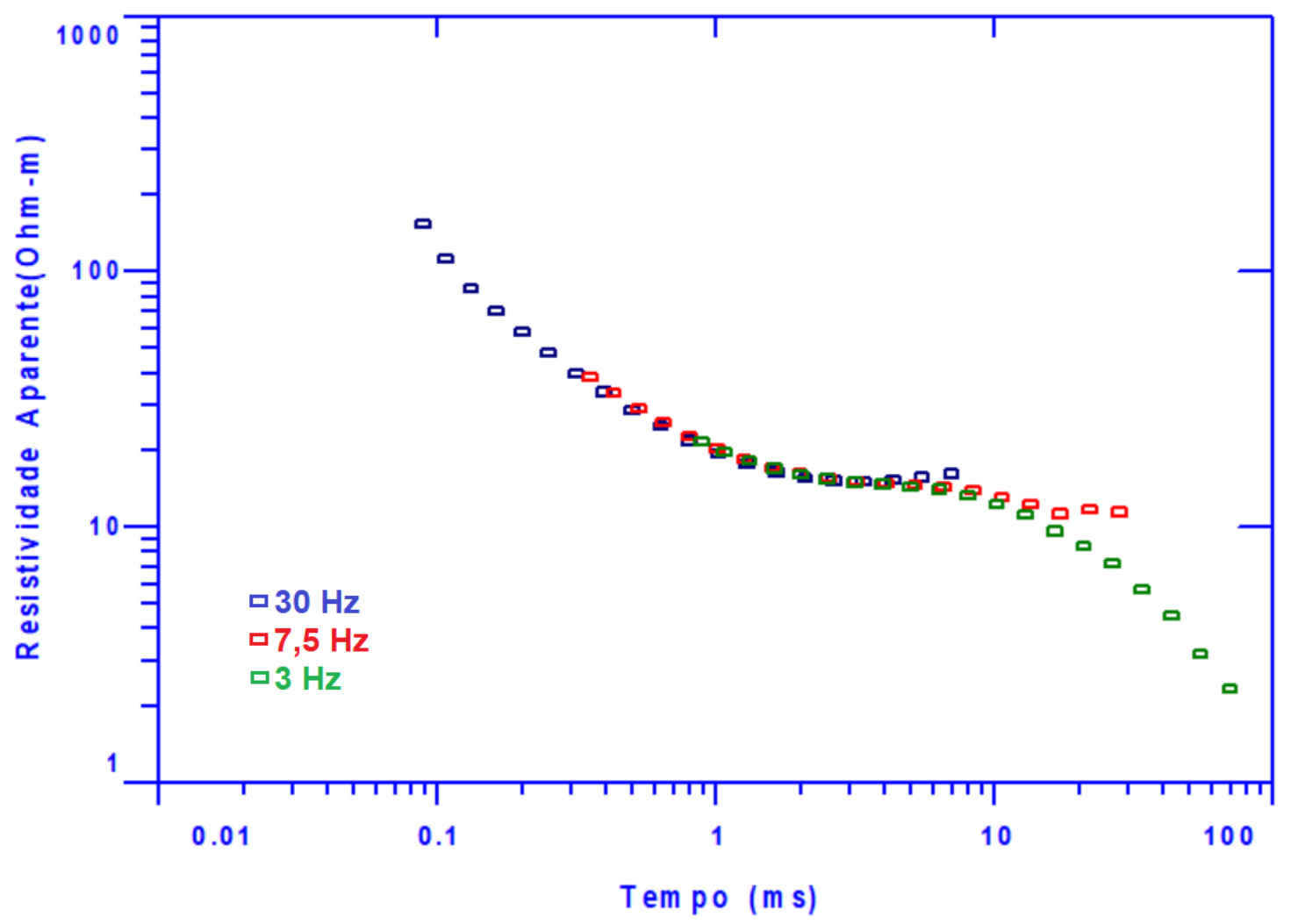

Figura 26 - Curva de dados TDEM brutos no software IX1D.

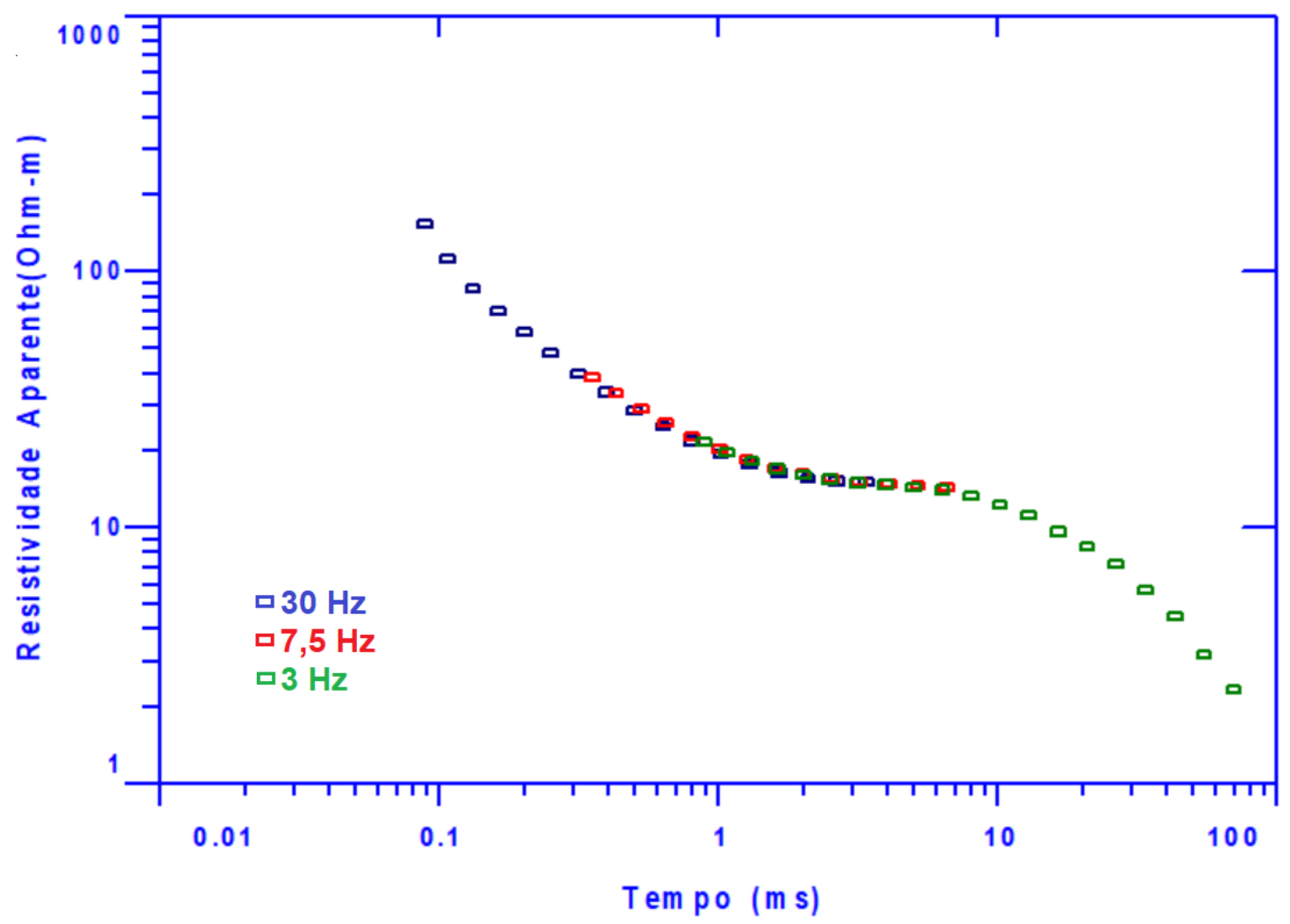

Figura 27 - Curva de dados TDEM editados no software IX1D. 
Após a edição dos dados passou-se a realizar as inversões individuais e conjuntas dos dados de CE e CTDEM. A Figura 24 apresenta a localização dos perfis de CE e CTDEM utilizados nas inversões, onde os 3 perfis de CTDEM, que coincidem com os perfis de inversão conjunta, estão numerados: 2 no loop vermelho com direções Leste-Oeste (1) e Norte-Sul (2) e 1 no loop azul com direção Norte-Sul (3).

As inversões individuais e conjuntas foram realizadas com as rotinas em MATLAB desenvolvidas por Bortolozo (2016), conforme a metodologia descrita no capítulo 4.

A Figura 28 apresenta a malha de diferenças finitas utilizada nas inversões individuais de CE, e a Figura 29 mostra um detalhe da malha. A Figura 30 apresenta o modelo geoelétrico inicial homogêneo, com resistividade igual a $100 \Omega \cdot \mathrm{m}$, utilizado nas inversões individuais de CE.

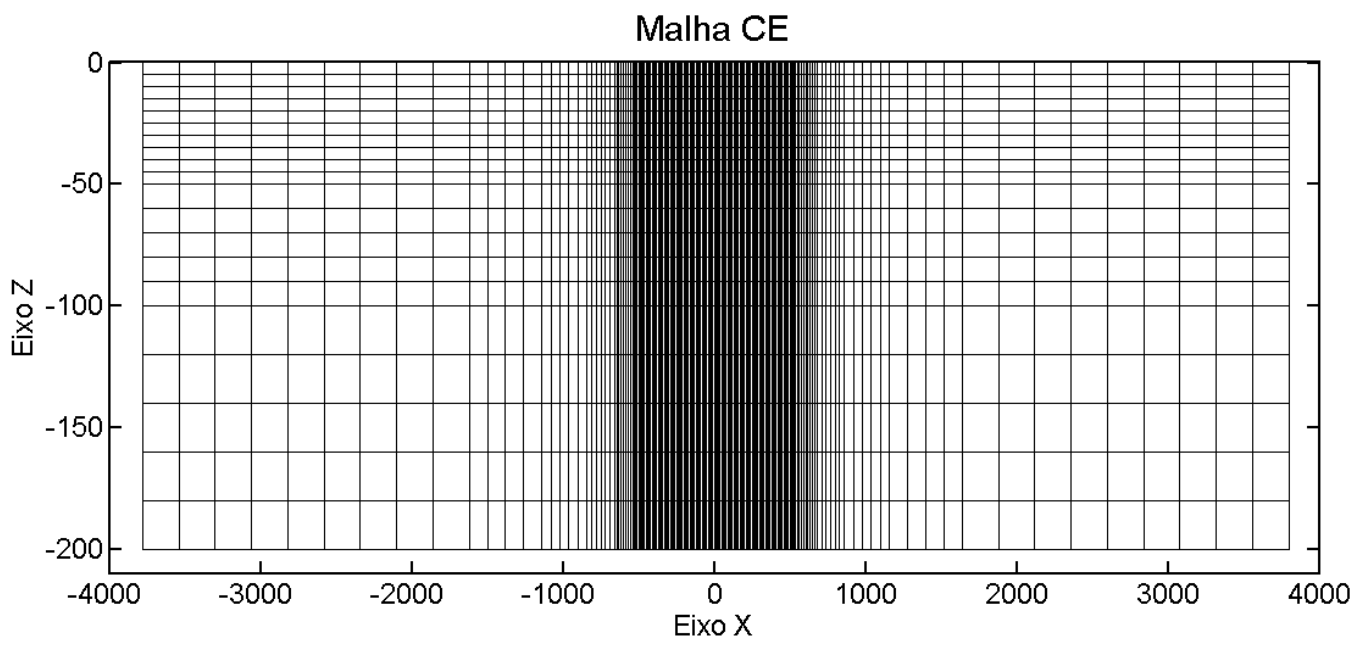

Figura 28 - Malha de diferenças finitas utilizada nas Inversões de CE.

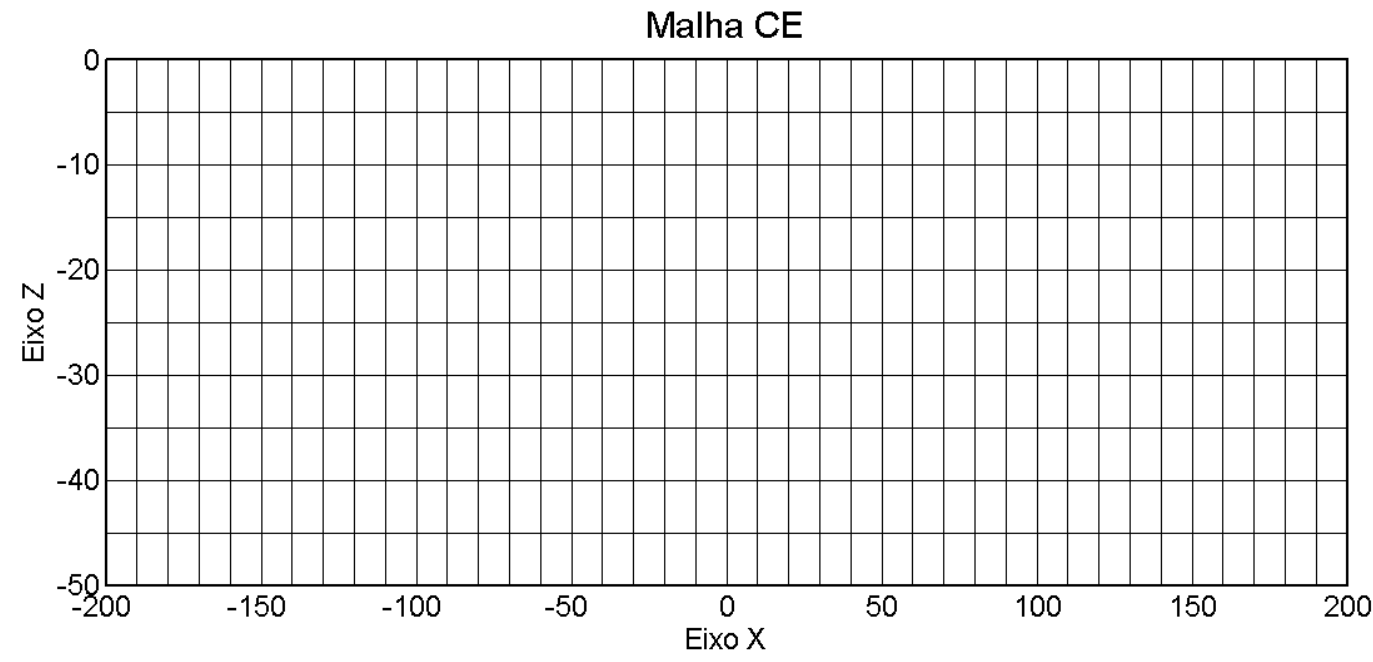

Figura 29 - Detalhe da malha de diferenças finitas da Figura 28. 


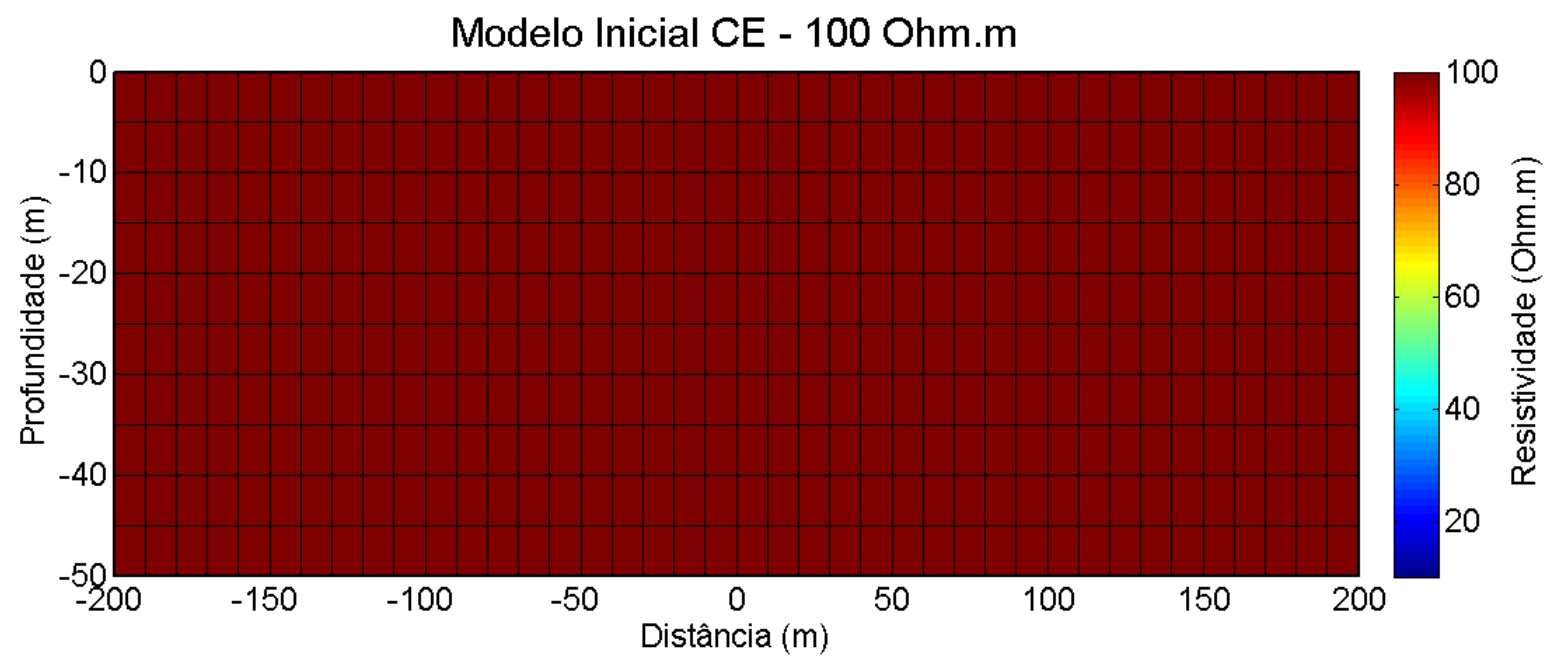

Figura 30 - Modelo geoelétrico inicial homogêneo com $100 \Omega \cdot m$, utilizado nas as Inversões de CE.

A Figura 31 apresenta a malha de diferenças finitas utilizada nas inversões individuais de CTDEM e inversões conjuntas CE/CTDEM, e a Figura 32 mostra um detalhe da malha. Já a Figura 33 apresenta o modelo geoelétrico inicial homogêneo, com resistividade igual a 30 $\Omega \cdot \mathrm{m}$, que foi utilizado nas inversões individuais de CTDEM e nas inversões conjuntas.

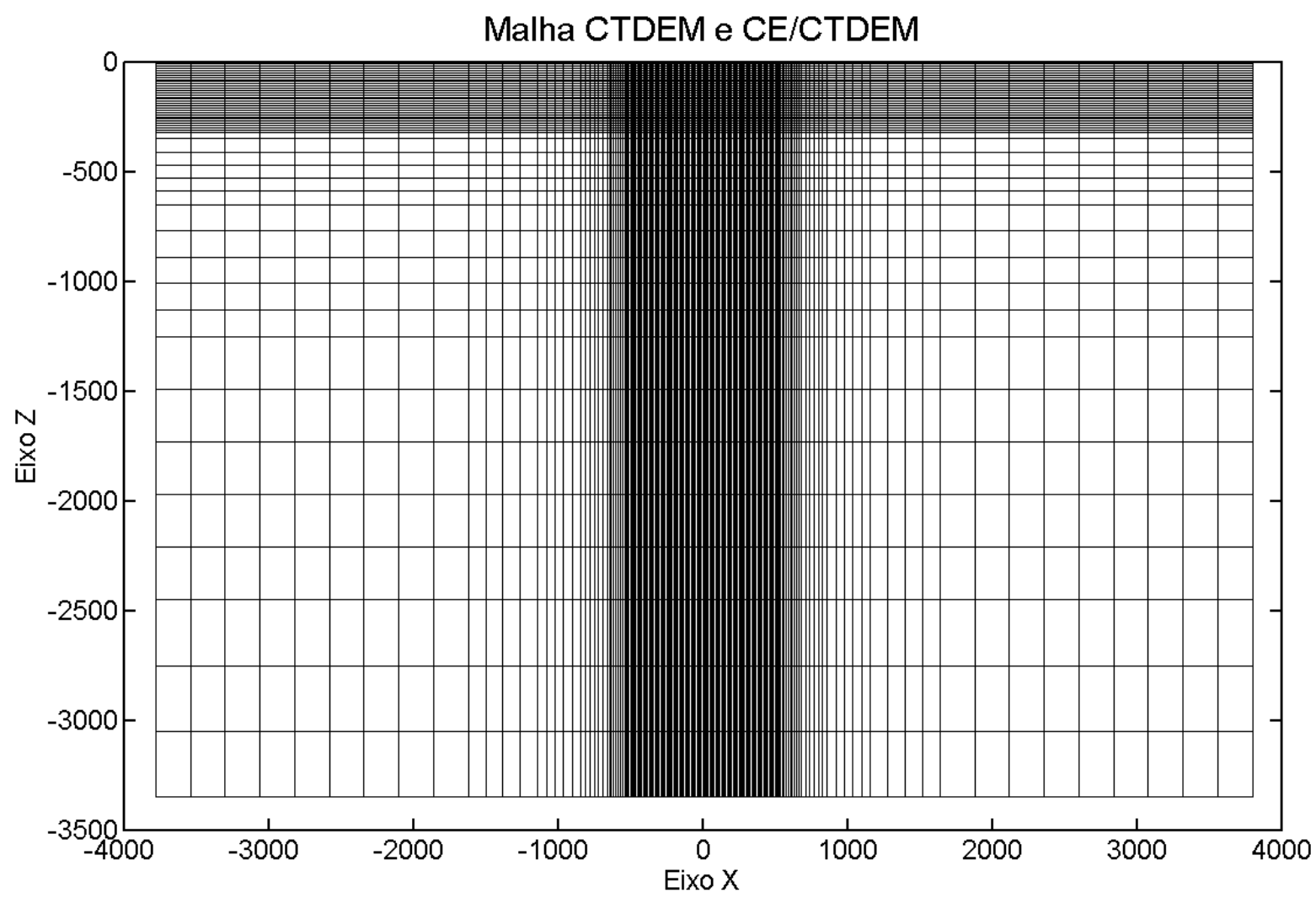

Figura 31 - Malha de diferenças finitas utilizada nas Inversões de CTDEM e nas Inversões Conjuntas 2D CE/CTDEM. 


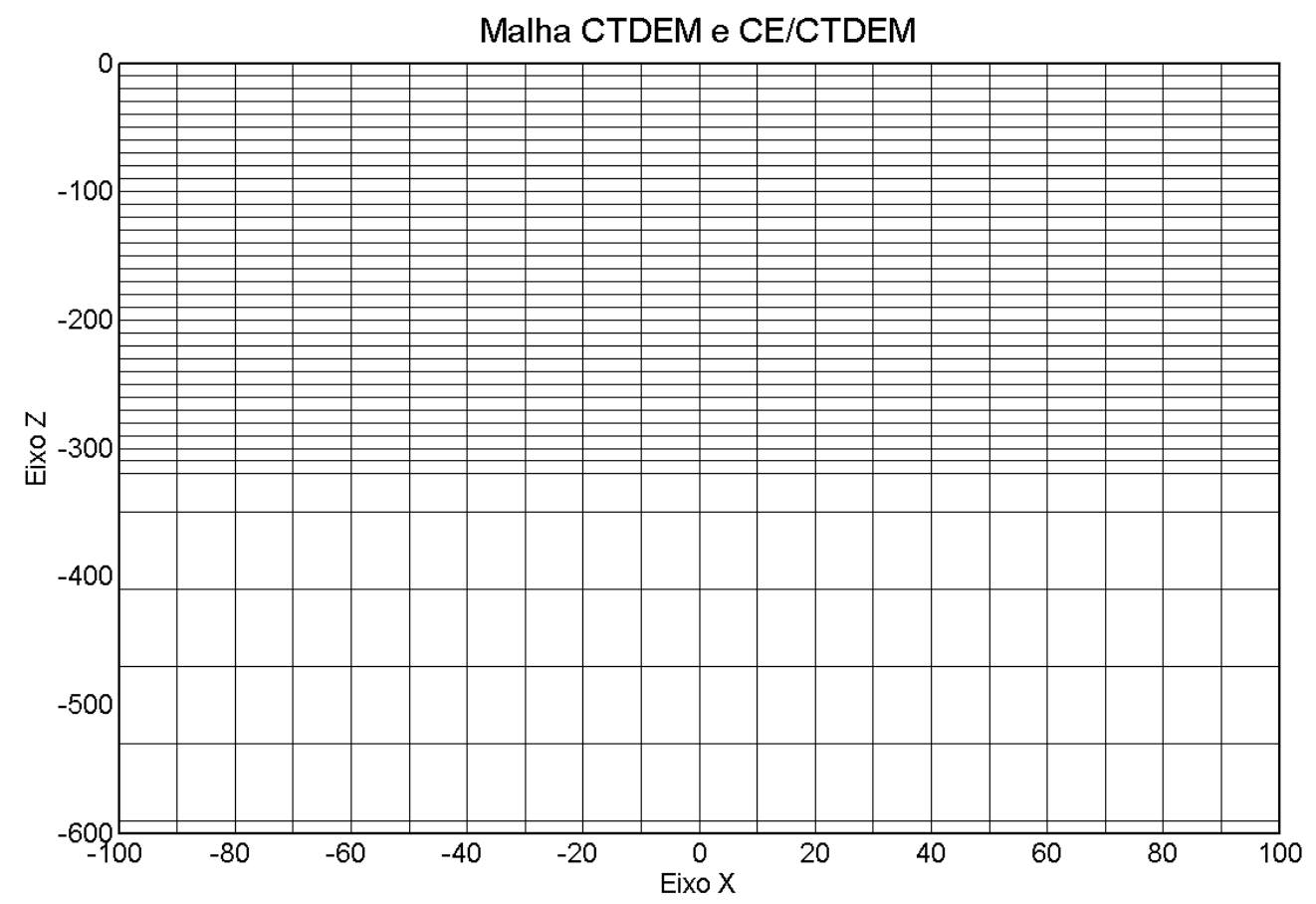

Figura 32 - Detalhe da malha de diferenças finitas da Figura 31.

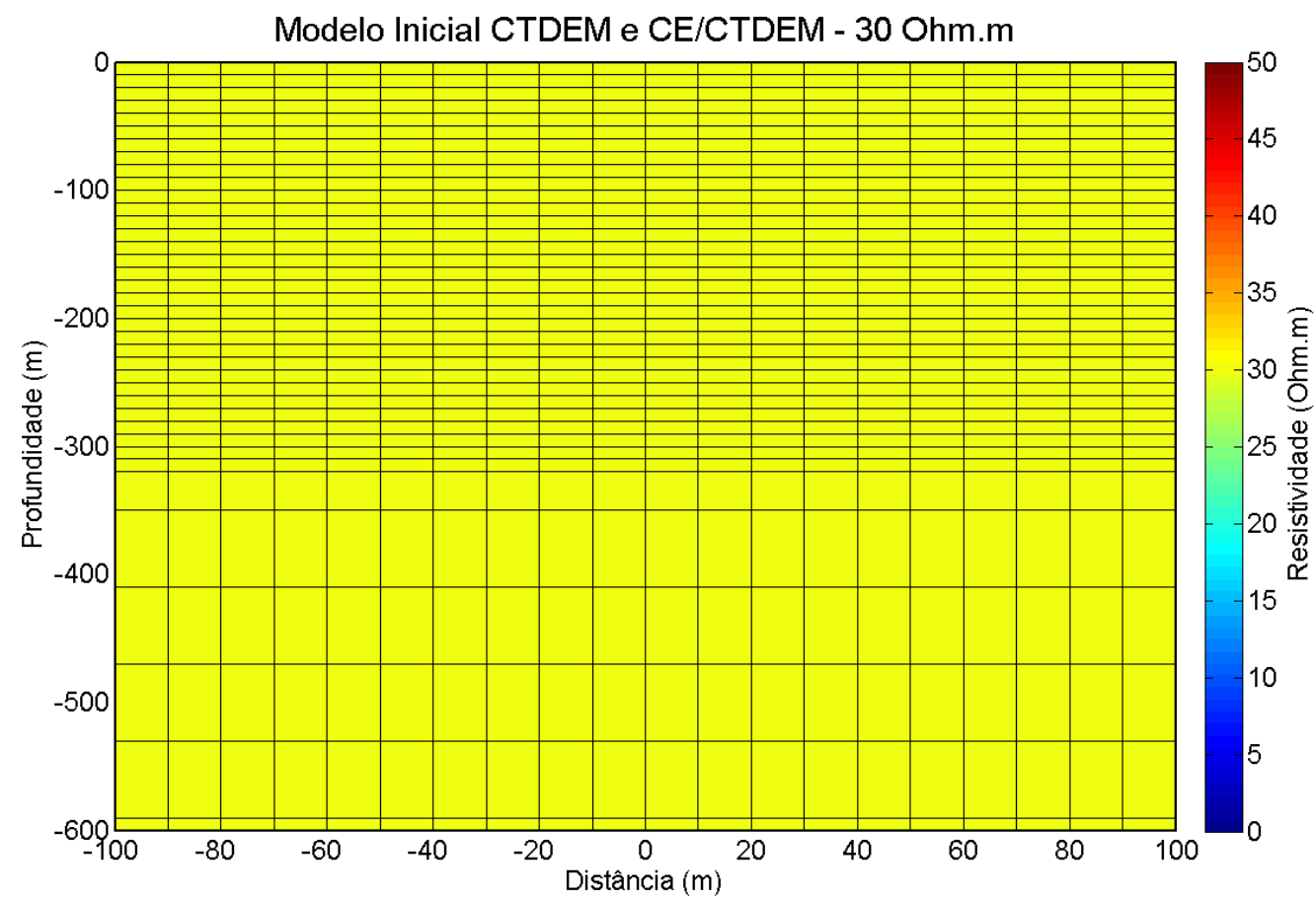

Figura 33 - Modelo geoelétrico inicial homogêneo com $30 \Omega \cdot m$, utilizado nas as Inversões de CTDEM e nas Inversões Conjuntas 2D CE/CTDEM.

As malhas foram definidas de uma forma para se adequar aos métodos geofísicos, sendo muito maior que a região de interesse, para atender as condições de fronteira necessárias para os cálculos das equações diferenciais de ambos os métodos. 


\section{RESULTADOS E INTERPRETAÇÕES}

Neste capítulo são apresentados os resultados e interpretações das Inversões Individuais de CE e CTDEM e das Inversões Conjuntas CE/CTDEM.

\subsection{Perfil 1}

As Figuras 34 e 35 apresentam os resultados das inversões dos CE's na direção do perfil 1 (E-W) (pontos azuis na Figura 24). Os dois CE's foram realizados com arranjo polodipolo, comprimento de $400 \mathrm{~m}$ e espaçamento de eletrodos de $10 \mathrm{~m}$ e $20 \mathrm{~m}$, respectivamente. Os erros de ajuste $(\Phi)$ das inversões foram de $15,1 \%$ e 17,5\%, respectivamente. A partir dos modelos geoelétricos invertidos (Figuras 34 e 35), observa-se que a resistividade diminui gradativamente com a profundidade e é possível identificar duas camadas geoelétricas. A primeira, com profundidade entre $40 \mathrm{~m}$ (leste) e $20 \mathrm{~m}$ (oeste), possui resistividade mais alta, atingindo valores maiores que $100 \Omega \cdot \mathrm{m}$, portanto, é uma camada resistiva. A segunda camada abaixo é condutiva, apresentando resistividade menor que $40 \Omega \cdot \mathrm{m}$.

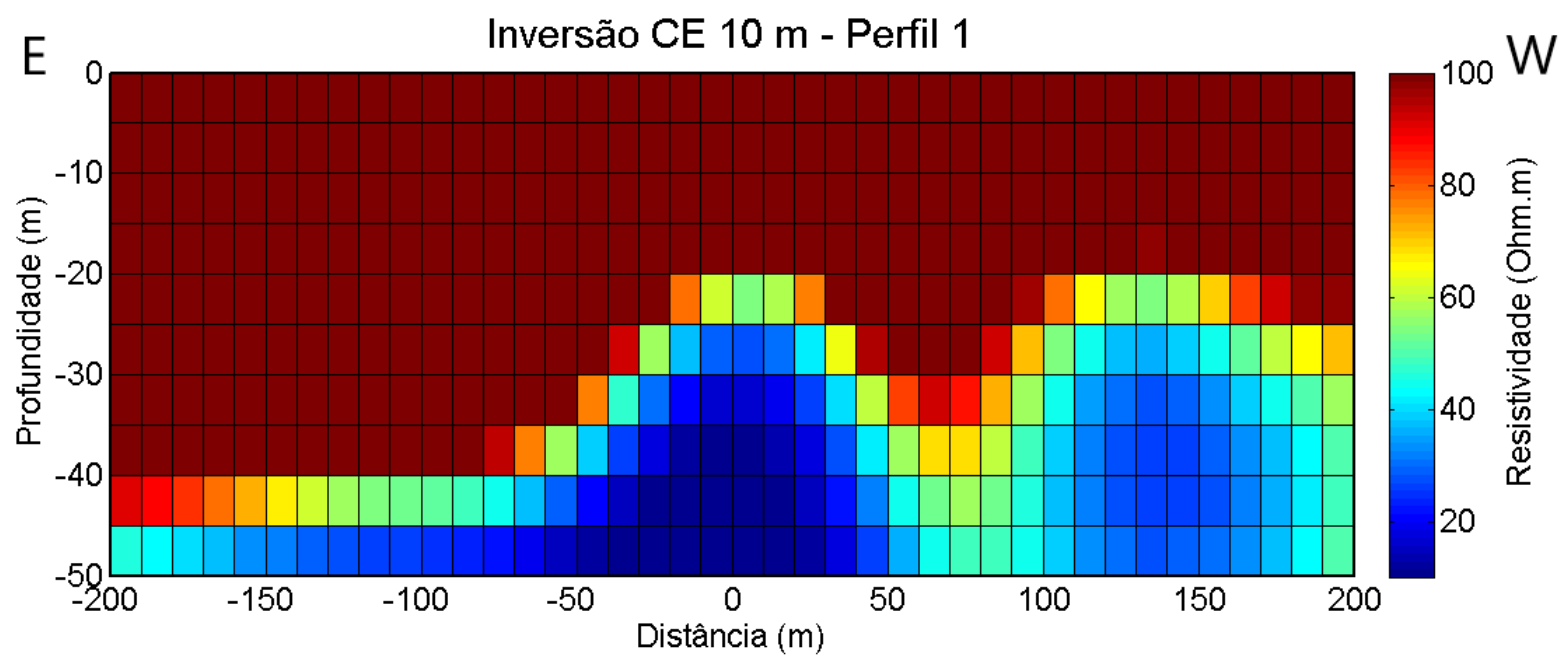

Figura 34 - Resultado da Inversão do CE com arranjo polo-dipolo e espaçamento de eletrodos de $10 \mathrm{~m}$ na direção do perfil 1 (E-W). 


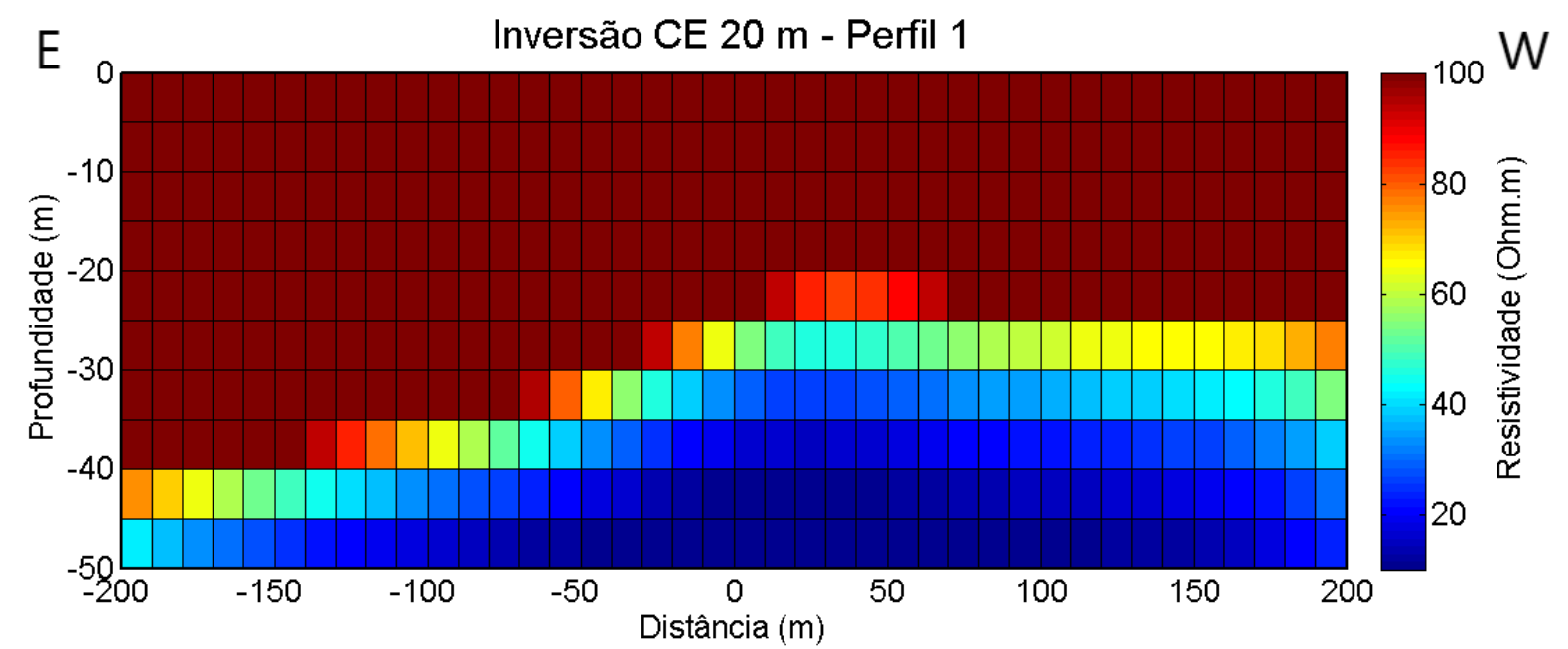

Figura 35 - Resultado da Inversão do CE com arranjo polo-dipolo e espaçamento de eletrodos de $20 \mathrm{~m}$ na direção do perfil 1 (E-W).

A Figura 36 apresenta o resultado da inversão individual do CTDEM na direção do perfil 1 (E-W) (Figura 24), onde o erro de ajuste obtido foi de 10,6\%. Embora a aquisição deste CTDEM tenha sido na direção Oeste para Leste (Figura 24), a inversão foi realizada na direção oposta, Leste para Oeste (Figura 36), para poder compará-la com a Inversão Conjunta CE/CTDEM do mesmo perfil (Figura 38). É possível observar 3 camadas geoelétricas. A primeira camada até a profundidade de $20 \mathrm{~m}$ apresenta resistividade em torno de $30 \Omega \cdot \mathrm{m}$, a segunda camada entre $20 \mathrm{~m}$ e $280 \mathrm{~m}$ de profundidade possui resistividade entre $5 \Omega \cdot \mathrm{m}$ e 20 $\Omega \cdot \mathrm{m}$ e a terceira camada, abaixo de $280 \mathrm{~m}$, possui resistividade entre $30 \Omega \cdot \mathrm{m}$ e $35 \Omega \cdot \mathrm{m}$. As camadas superficiais são mais resistivas, por isso não foram bem determinadas quando comparadas com os resultados das Inversões do CE (Figuras 34 e 35), pois o método TDEM possui baixa resolução para as camadas mais rasas. No entanto, o resultado da inversão do CTDEM do perfil 1 (Figura 36) permitiu identificar uma camada condutiva entre $20 \mathrm{~m}$ e 280 $\mathrm{m}$ de profundidade. A terceira camada, abaixo de $280 \mathrm{~m}$ profundidade, provavelmente está no limite de resolução do método TDEM, essa camada também é observada nas inversões conjuntas e será discutida na interpretação dos resultados (seção 6.4). 


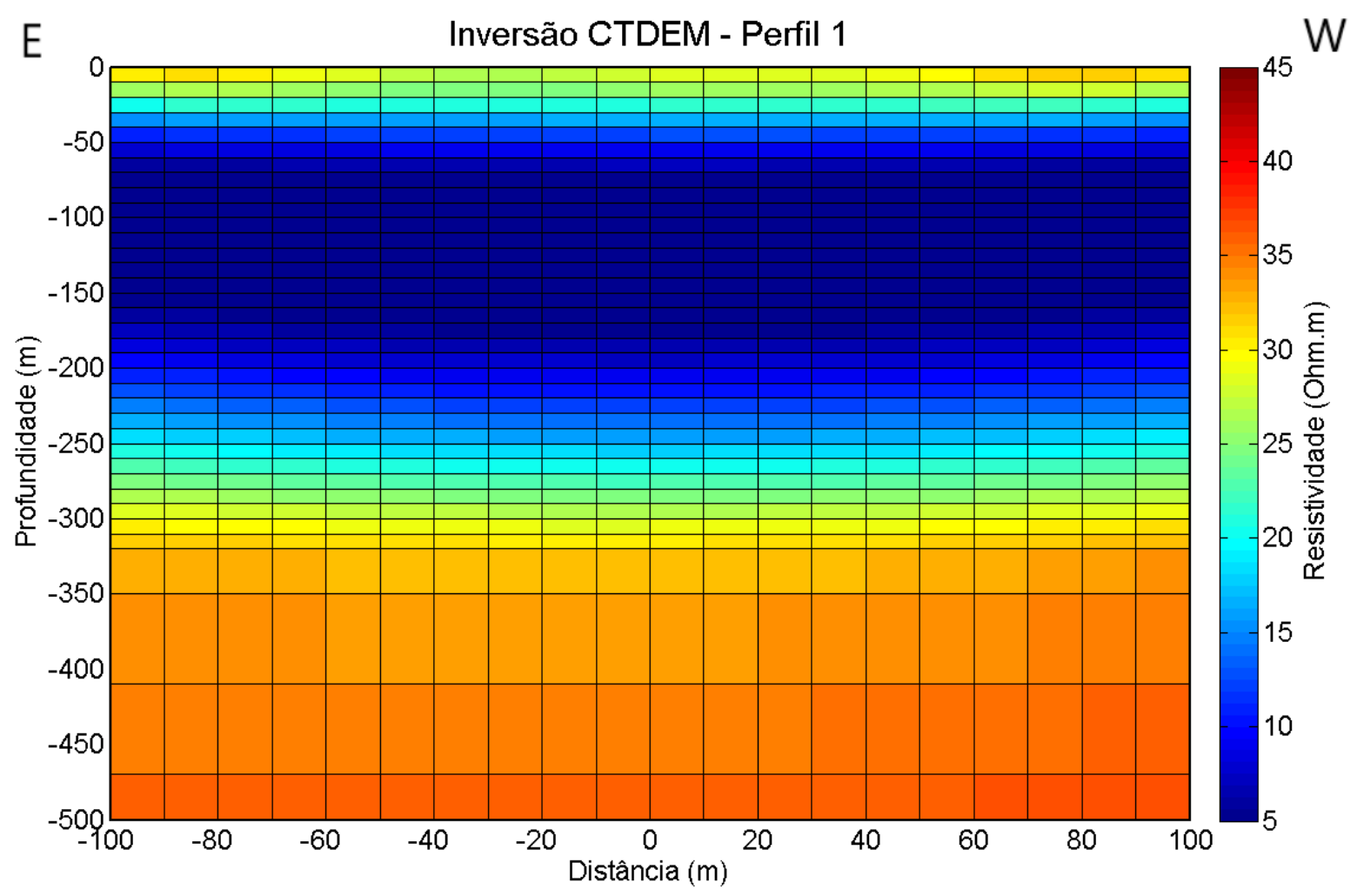

Figura 36 - Resultado da Inversão do CTDEM na direção do perfil 1 (E-W).

A Figura 37 apresenta o modelo geoelétrico obtido a partir da Inversão Conjunta 2D CE/CTDEM do perfil 1 (E-W) com a escala de cor entre $10 \Omega \cdot \mathrm{m}$ e $100 \Omega \cdot \mathrm{m}$, onde é possível observar melhor as camadas resistivas mais rasas, e a Figura 38 apresenta o mesmo resultado com a escala de cor entre $5 \Omega \cdot \mathrm{m}$ e $45 \Omega \cdot \mathrm{m}$, onde é possível observar melhor o contraste em $300 \mathrm{~m}$ de profundidade. O erro de ajuste da inversão foi de 10,9\%. É possível observar 4 camadas geoelétricas. A primeira camada com até $30 \mathrm{~m}$ de profundidade, possui resistividade entre $45 \Omega \cdot \mathrm{m}$ e valores maiores que $100 \Omega \cdot \mathrm{m}$. A segunda camada abaixo, possui $20 \mathrm{~m}$ de espessura e resistividade média de aproximadamente $40 \Omega \cdot \mathrm{m}$. A terceira camada entre $50 \mathrm{~m}$ e $300 \mathrm{~m}$, é muito condutiva, com resistividade entre $5 \Omega \cdot \mathrm{m}$ e $20 \Omega \cdot \mathrm{m}$. Abaixo de $300 \mathrm{~m}$ de profundidade a resistividade é de aproximadamente $20 \Omega \cdot \mathrm{m}$. 


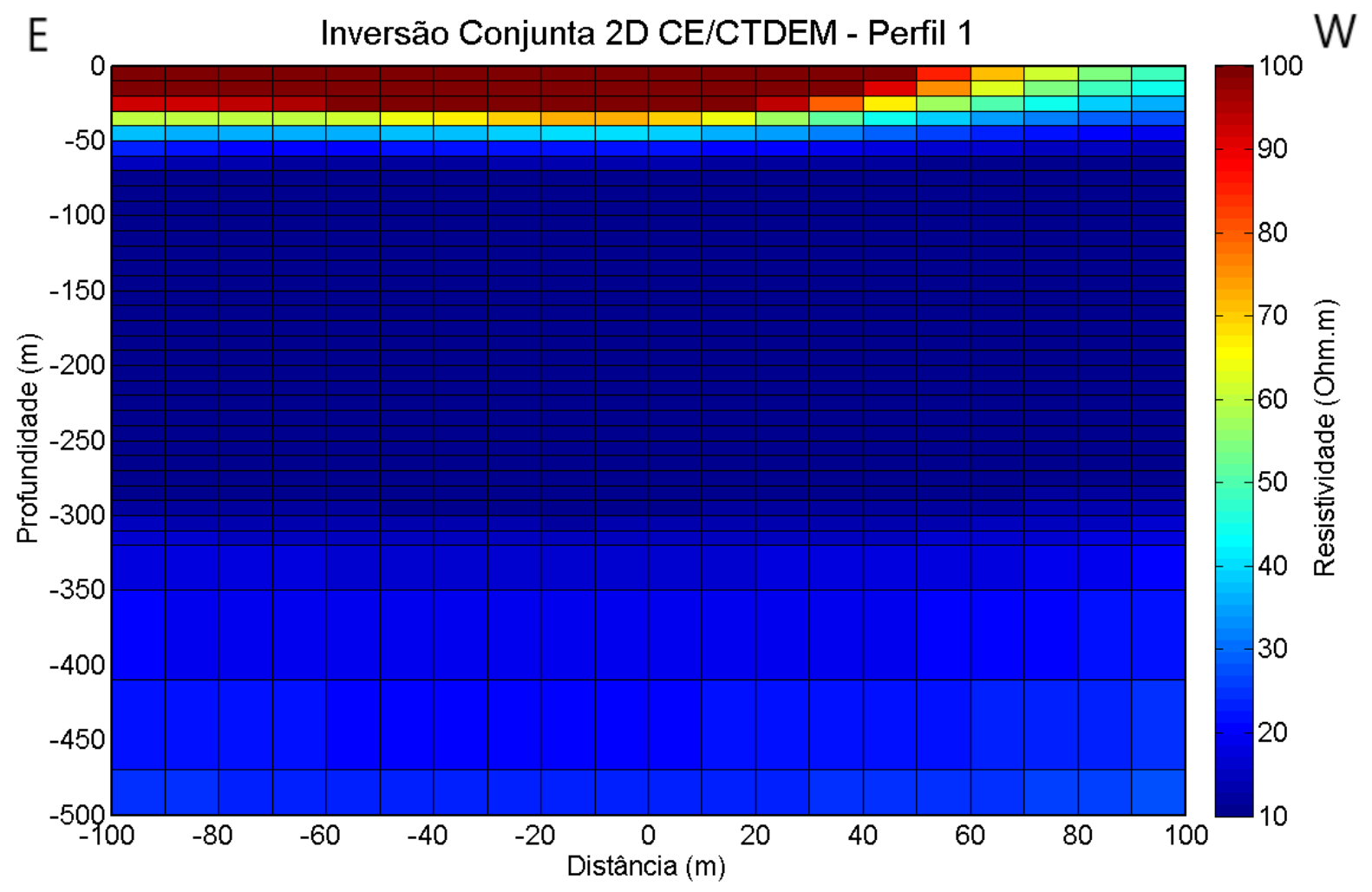

Figura 37 - Resultado da Inversão Conjunta 2D CE/CTDEM para o perfil 1 (E-W) com a escala de cores entre $10 \Omega \cdot \mathrm{m}$ e $100 \Omega \cdot \mathrm{m}$.

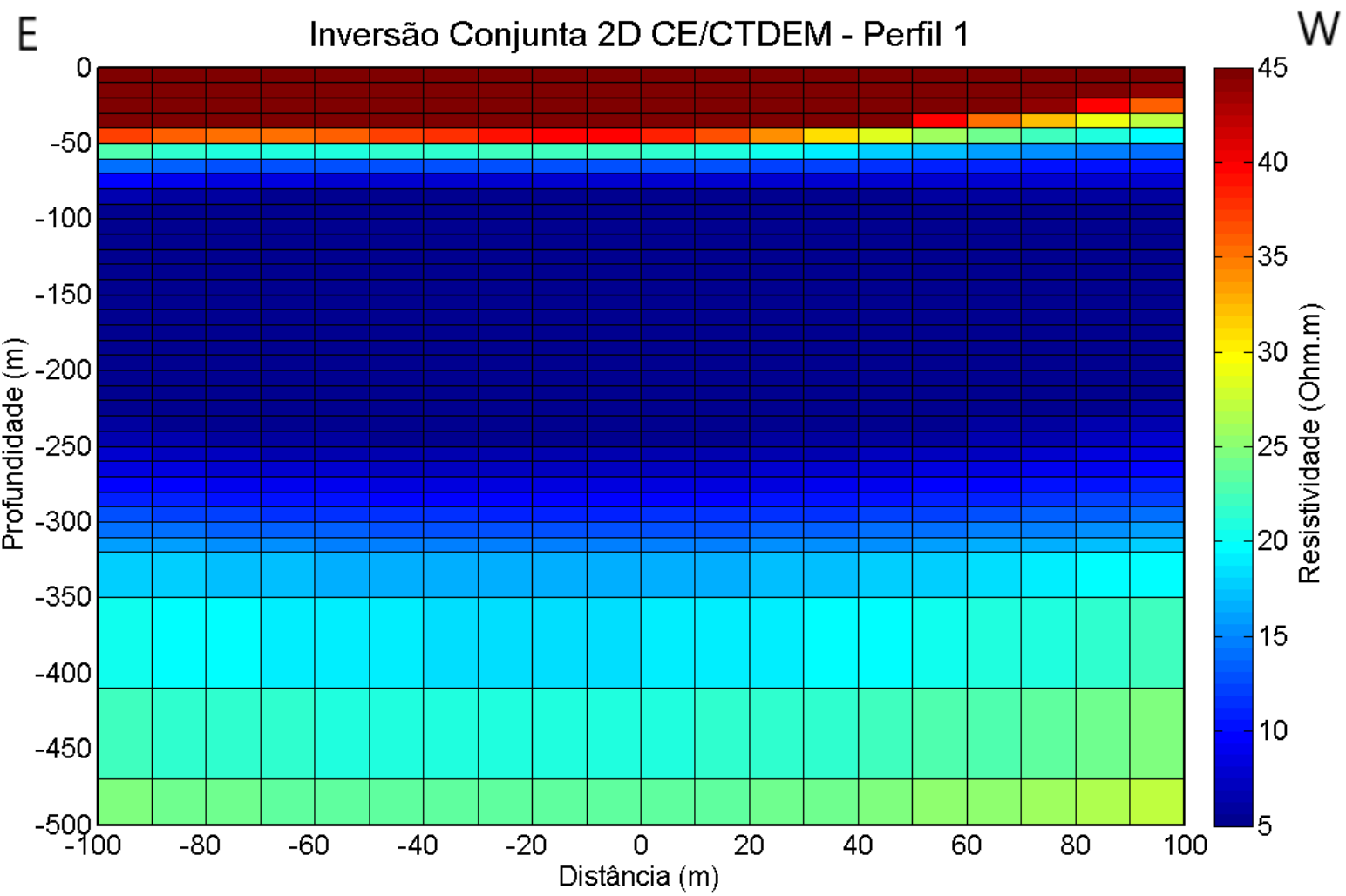

Figura 38 - Resultado da Inversão Conjunta 2D CE/CTDEM para o perfil 1 (E-W) com a escala de cores entre $5 \Omega \cdot m$ e $45 \Omega \cdot m$. 


\subsection{Perfil 2}

As Figura 39 apresenta o resultado da inversão do CE na direção do perfil 2 (N-S) (pontos verdes na Figura 24), realizado com espaçamento de eletrodos de $10 \mathrm{~m}$, arranjo polodipolo e comprimento de $400 \mathrm{~m}$ na direção Norte-Sul. O erro de ajuste foi de $20,0 \%$. É possível identificar duas camadas geoelétricas: a primeira entre $25 \mathrm{~m}$ e $35 \mathrm{~m}$ de profundidade é resistiva apresentando resistividade maior que $100 \Omega \cdot \mathrm{m}$, e, abaixo, a segunda camada é condutiva, com resistividade menor que $40 \Omega \cdot \mathrm{m}$.

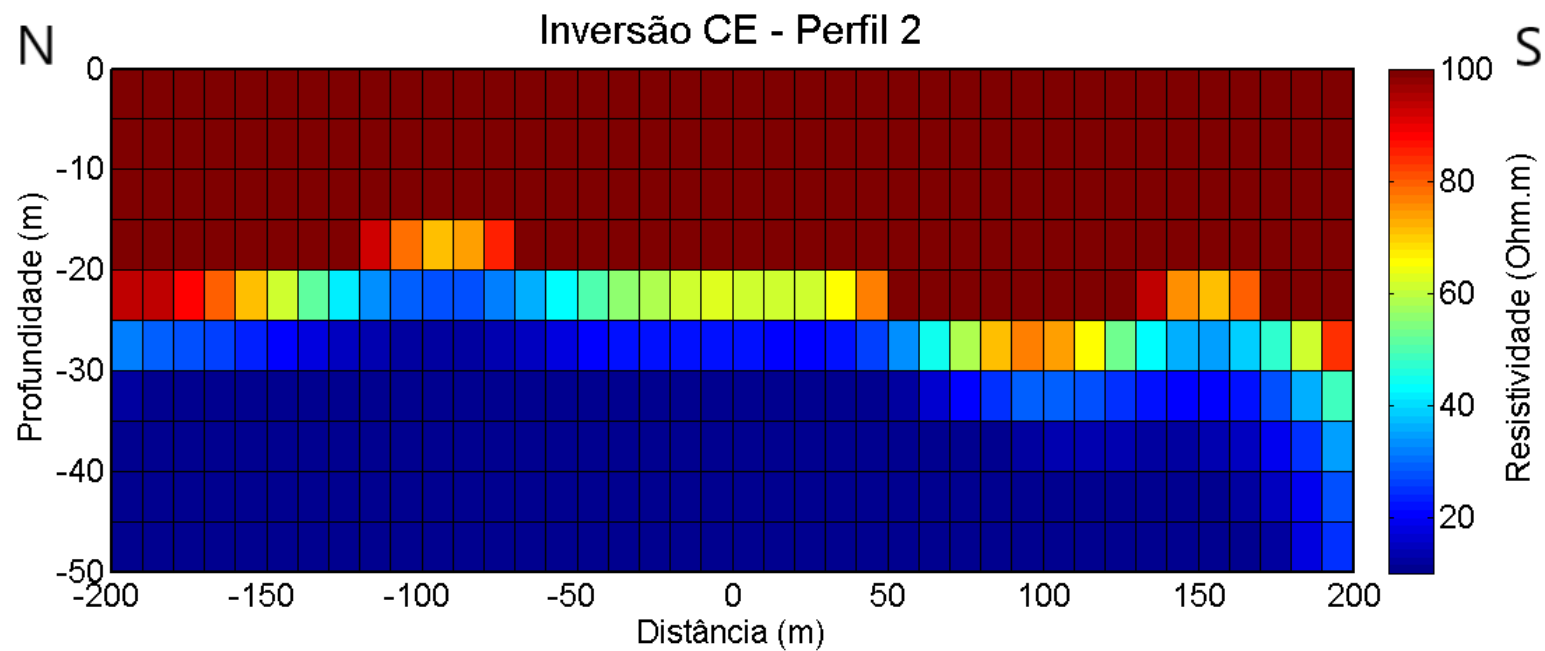

Figura 39 - Resultado da Inversão do CE com arranjo polo-dipolo e espaçamento de eletrodos de $10 \mathrm{~m}$ na direção do perfil $2(\mathrm{~N}-\mathrm{S})$.

A Figura 40 apresenta o resultado da inversão do CTDEM na direção do perfil 2, onde o erro de ajuste foi de 10,7\%. Embora a aquisição deste CTDEM tenha sido na direção Sul para Norte (Figura 24), a inversão foi realizada na direção oposta, Norte para Sul (Figura 40), para poder compará-la com a Inversão Conjunta CE/CTDEM do mesmo perfil (Figura 42). Na Figura 40 é possível observar 3 camadas geoelétricas: a primeira até a profundidade de 20 $\mathrm{m}$ apresenta resistividade em torno de $30 \Omega \cdot \mathrm{m}$, a segunda camada entre $20 \mathrm{~m}$ e $280 \mathrm{~m}$ de profundidade possui resistividade entre $5 \Omega \cdot \mathrm{m}$ e $20 \Omega \cdot \mathrm{m}$ e a terceira camada, abaixo de $280 \mathrm{~m}$, possui resistividade entre $30 \Omega \cdot \mathrm{m}$ e $40 \Omega \cdot \mathrm{m}$. 


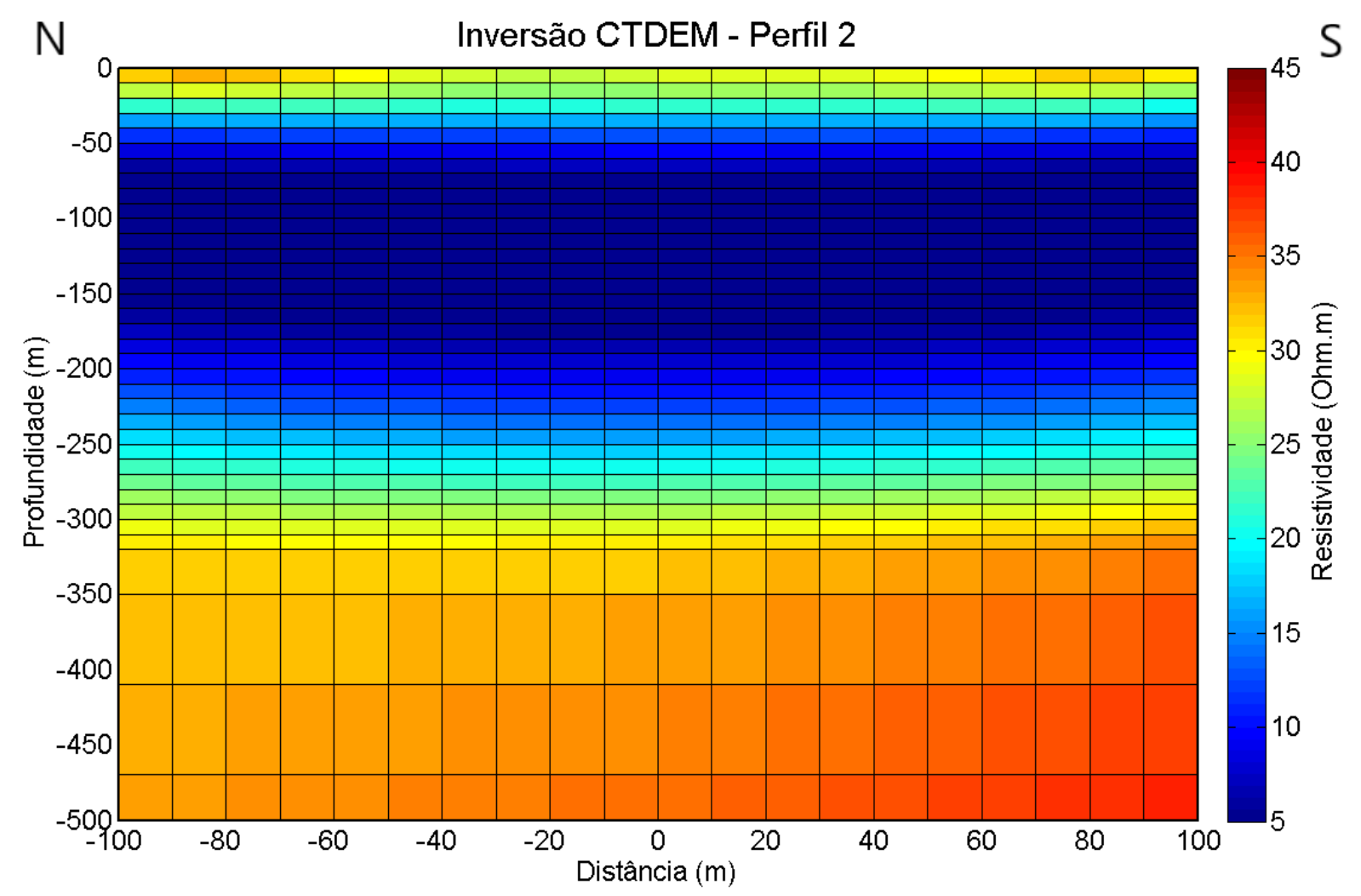

Figura 40 - Resultado da Inversão do CTDEM na direção do perfil 2 (N-S).

A Figura 41 apresenta o modelo geoelétrico obtido a partir da Inversão Conjunta 2D CE/CTDEM do perfil $2(\mathrm{~N}-\mathrm{S})$ com a escala de cor entre $10 \Omega \cdot \mathrm{m}$ e $100 \Omega \cdot \mathrm{m}$, onde é possível observar melhor as camadas resistivas mais rasas, e a Figura 42 apresenta o mesmo resultado com a escala de cor entre $5 \Omega \cdot \mathrm{m}$ e $45 \Omega \cdot \mathrm{m}$, onde é possível observar melhor o contraste em $300 \mathrm{~m}$ de profundidade. O erro de ajuste da inversão foi de 14,0\%. É possível identificar 4 camadas geoelétricas: a primeira camada com até $30 \mathrm{~m}$ de profundidade, possui resistividade entre $45 \Omega \cdot \mathrm{m}$ e valores maiores que $100 \Omega \cdot \mathrm{m}$; a segunda camada com espessura de $20 \mathrm{~m}$ apresenta resistividade média de aproximadamente $40 \Omega \cdot \mathrm{m}$; a terceira camada entre $50 \mathrm{~m}$ e $300 \mathrm{~m}$ é muito condutiva, com resistividade entre $5 \Omega \cdot \mathrm{m}$ e $20 \Omega \cdot \mathrm{m}$; e abaixo de $300 \mathrm{~m}$ de profundidade a resistividade aumenta para valores entre $20 \Omega \cdot \mathrm{m}$ e $30 \Omega \cdot \mathrm{m}$. 


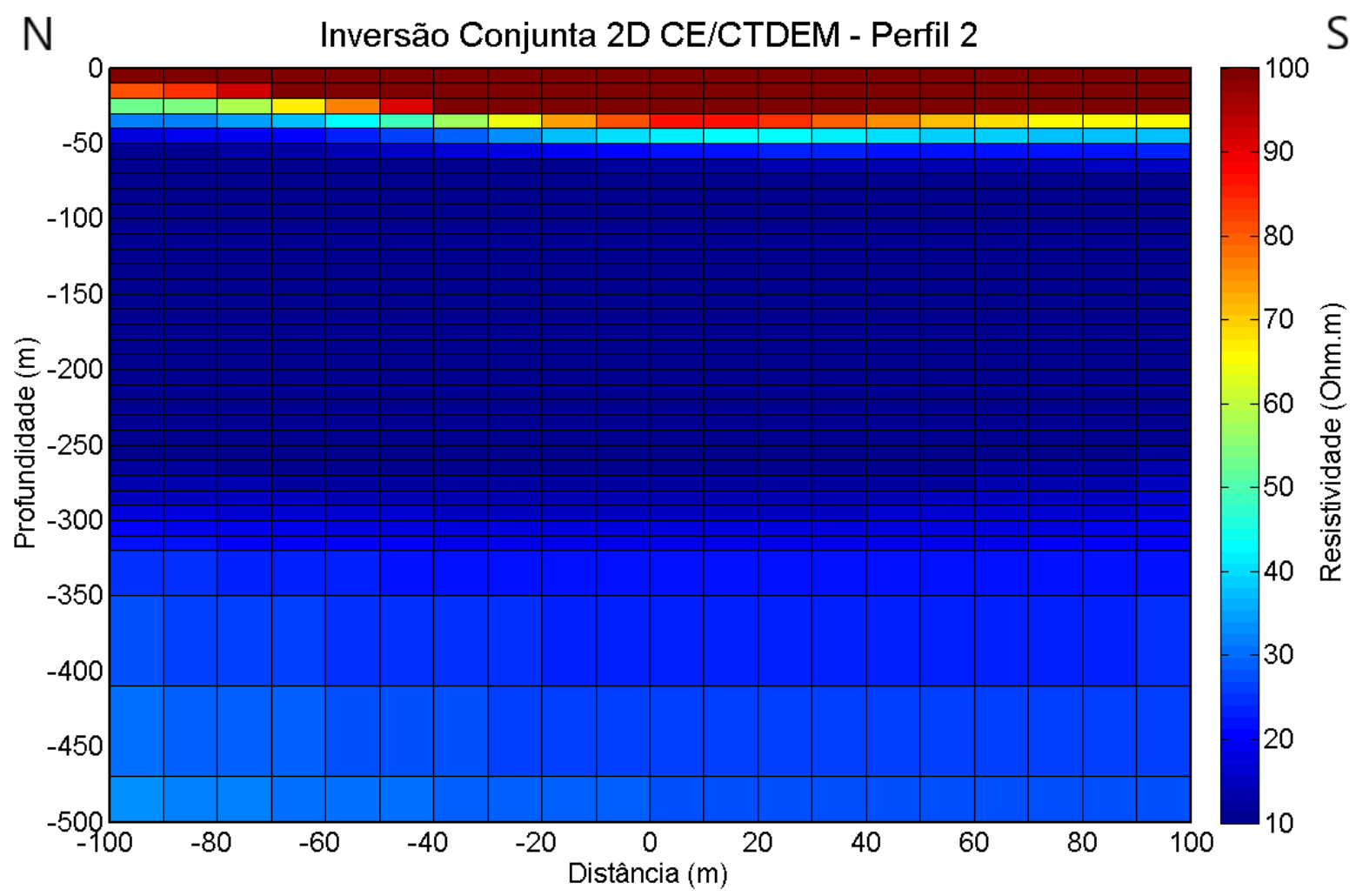

Figura 41 - Resultado da Inversão Conjunta 2D CE/CTDEM para o perfil 2 (N-S) com a escala de cores entre $10 \Omega \cdot m$ e $100 \Omega \cdot m$.

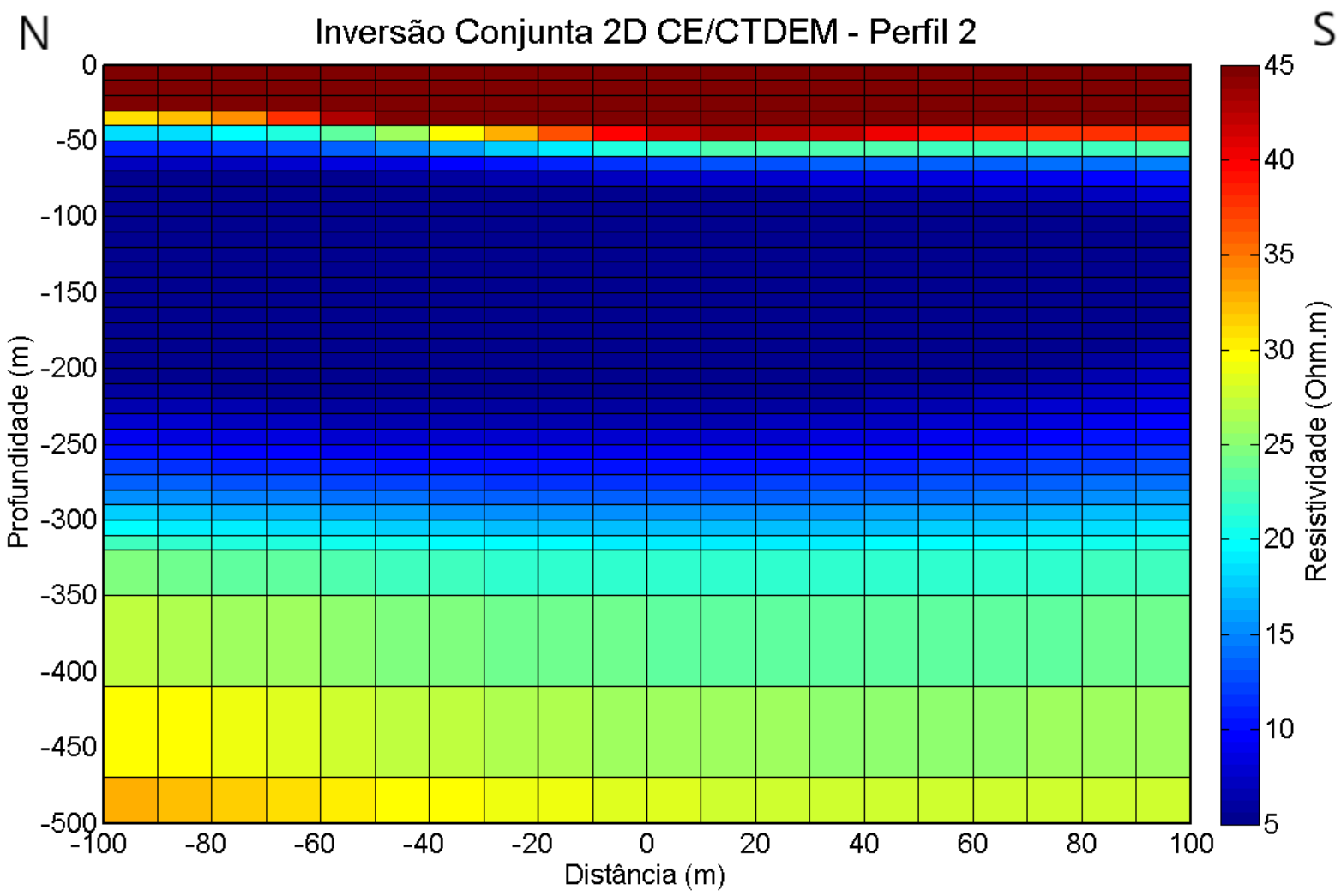

Figura 42 - Resultado da Inversão Conjunta 2D CE/CTDEM para o perfil 2 (N-S) com a escala de cores entre $5 \Omega \cdot \mathrm{m}$ e $45 \Omega \cdot \mathrm{m}$. 


\subsection{Perfil 3}

As Figura 43 apresenta o resultado da inversão do CE na direção do perfil 3 (N-S) (pontos amarelos na Figura 24), realizado com espaçamento de eletrodos de $20 \mathrm{~m}$, arranjo dipolo-dipolo e comprimento de $660 \mathrm{~m}$ na direção Norte-Sul. O erro de ajuste foi de $12,5 \%$. É possível identificar duas camadas geoelétricas: a primeira entre $20 \mathrm{~m}$ e $30 \mathrm{~m}$ de profundidade é resistiva apresentando resistividade maior que $100 \Omega \cdot \mathrm{m}$, e, abaixo, a segunda camada é condutiva, com resistividade menor que $40 \Omega \cdot \mathrm{m}$.

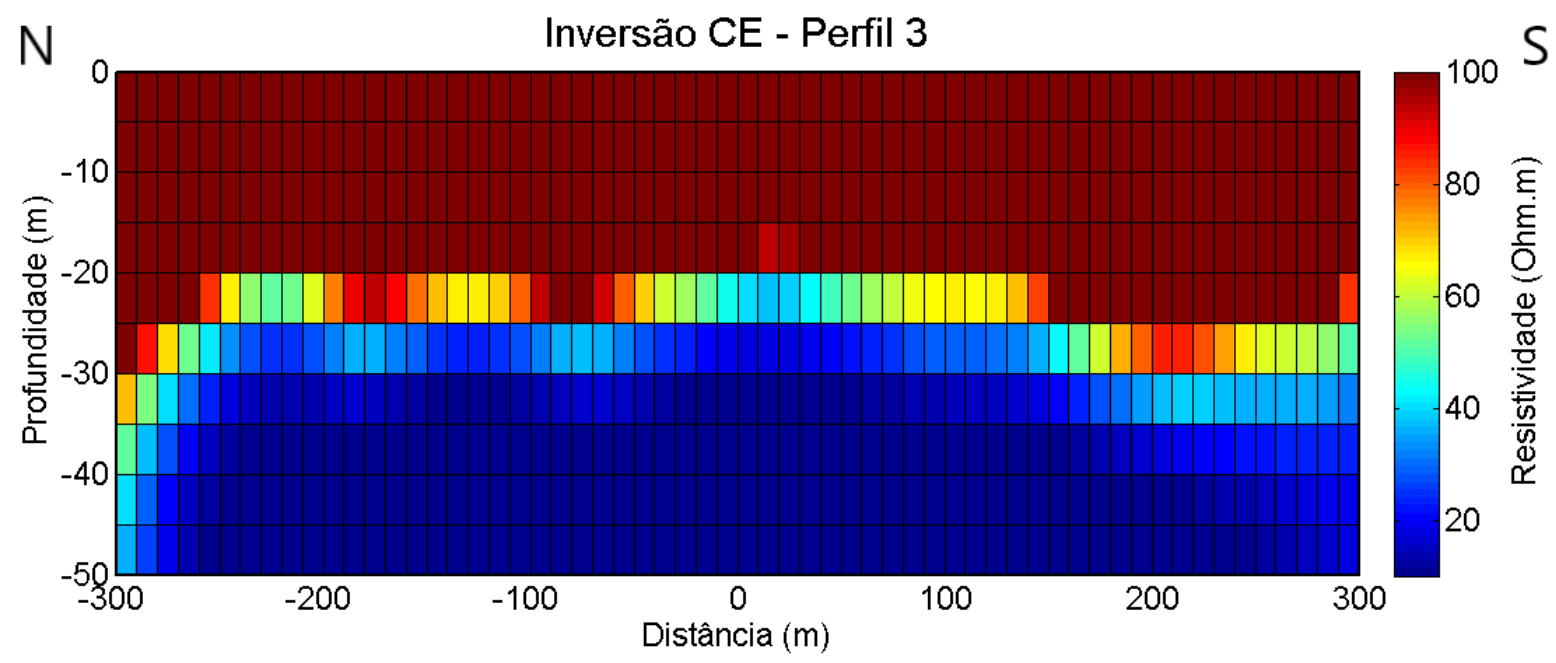

Figura 43 - Resultado da Inversão do CE com arranjo dipolo-dipolo e espaçamento de eletrodos de 20 m na direção do perfil $3(\mathrm{~N}-\mathrm{S})$.

A Figura 44 apresenta o resultado da inversão do CTDEM na direção do perfil 3, onde o erro de ajuste foi de 9,7\%. A aquisição deste CTDEM foi na direção Sul para Norte (Figura 24), no entanto, a inversão foi realizada na direção oposta, Norte para Sul (Figura 44), para poder compará-la com a Inversão Conjunta CE/CTDEM do mesmo perfil (Figura 46). Na Figura 44 é possível observar 3 camadas geoelétricas: a primeira até a profundidade de $30 \mathrm{~m}$ apresenta resistividade entre $25 \Omega \cdot \mathrm{m}$ e $45 \Omega \cdot \mathrm{m}$, a segunda entre $30 \mathrm{~m}$ e $300 \mathrm{~m}$ de profundidade possui resistividade entre $5 \Omega \cdot \mathrm{m}$ e $20 \Omega \cdot \mathrm{m}$ e a terceira camada, abaixo de $300 \mathrm{~m}$, possui resistividade entre $20 \Omega \cdot \mathrm{m}$ e $25 \Omega \cdot \mathrm{m}$. 


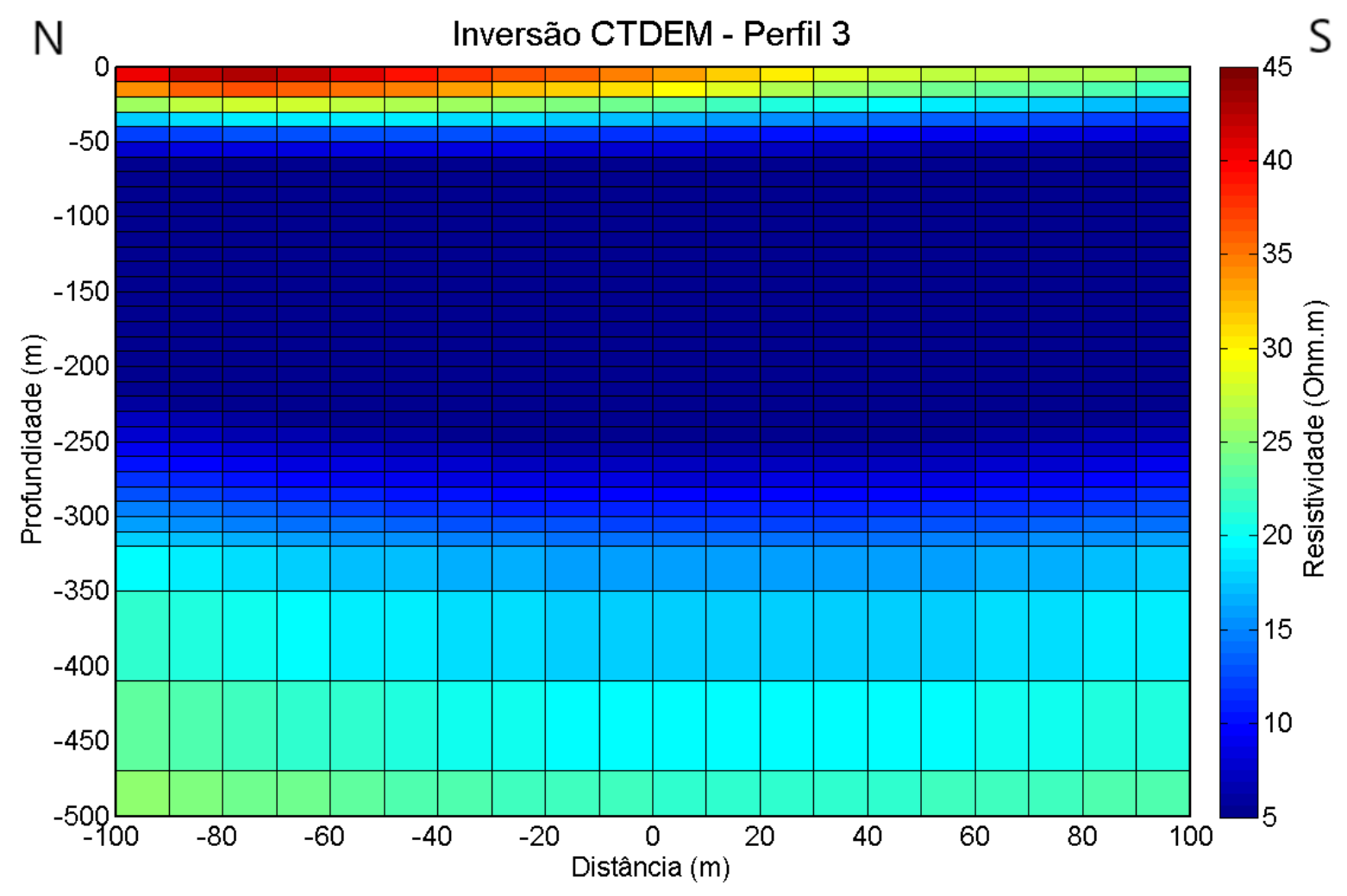

Figura 44 - Resultado da Inversão do CTDEM na direção do perfil 3 (N-S).

A Figura 45 apresenta o modelo geoelétrico obtido a partir da Inversão Conjunta 2D CE/CTDEM do perfil $3(\mathrm{~N}-\mathrm{S})$ com a escala de cor entre $10 \Omega \cdot \mathrm{m}$ e $100 \Omega \cdot \mathrm{m}$, onde é possível observar melhor as camadas resistivas mais rasas, e a Figura 46 apresenta o mesmo resultado com a escala de cor entre $5 \Omega \cdot \mathrm{m}$ e $45 \Omega \cdot \mathrm{m}$, onde é possível observar melhor o contraste em $300 \mathrm{~m}$ de profundidade. O erro de ajuste da inversão foi de 13,2\%. É possível identificar 4 camadas geoelétricas: a primeira camada com $30 \mathrm{~m}$ de profundidade, possui resistividade entre $45 \Omega \cdot \mathrm{m}$ e valores maiores que $100 \Omega \cdot \mathrm{m}$; a segunda camada com espessura de $20 \mathrm{~m}$ possui resistividade de aproximadamente $40 \Omega \cdot \mathrm{m}$; a terceira camada entre $50 \mathrm{~m}$ e $300 \mathrm{~m}$ é muito condutiva, com resistividade entre $5 \Omega \cdot \mathrm{m}$ e $20 \Omega \cdot \mathrm{m}$; e abaixo de $300 \mathrm{~m}$ de profundidade a resistividade aumenta para valores entre $20 \Omega \cdot \mathrm{m}$ e $35 \Omega \cdot \mathrm{m}$. 


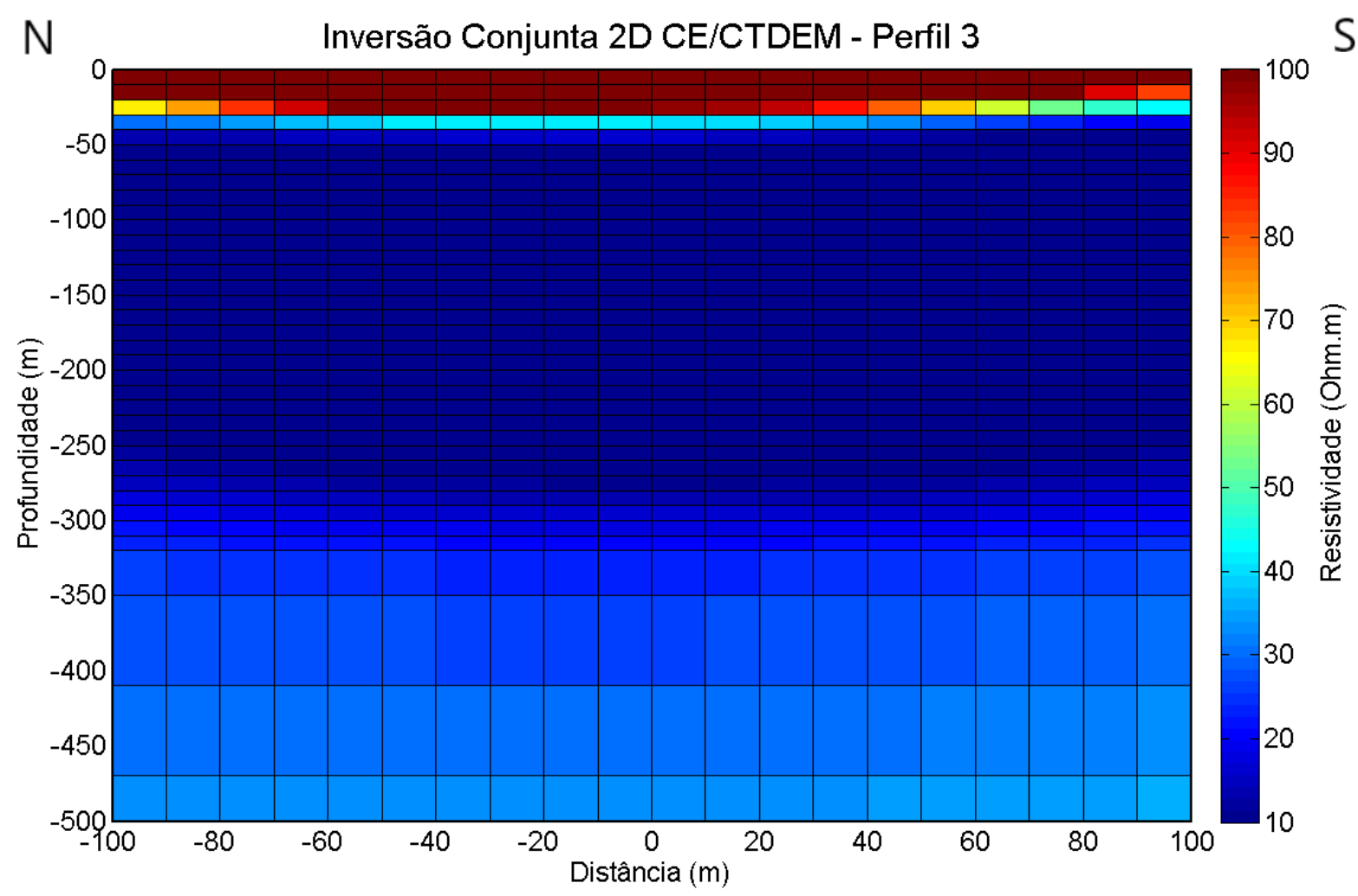

Figura 45 - Resultado da Inversão Conjunta 2D CE/CTDEM para o perfil 3 (N-S) com a escala de cores entre $10 \Omega \cdot m$ e $100 \Omega \cdot m$

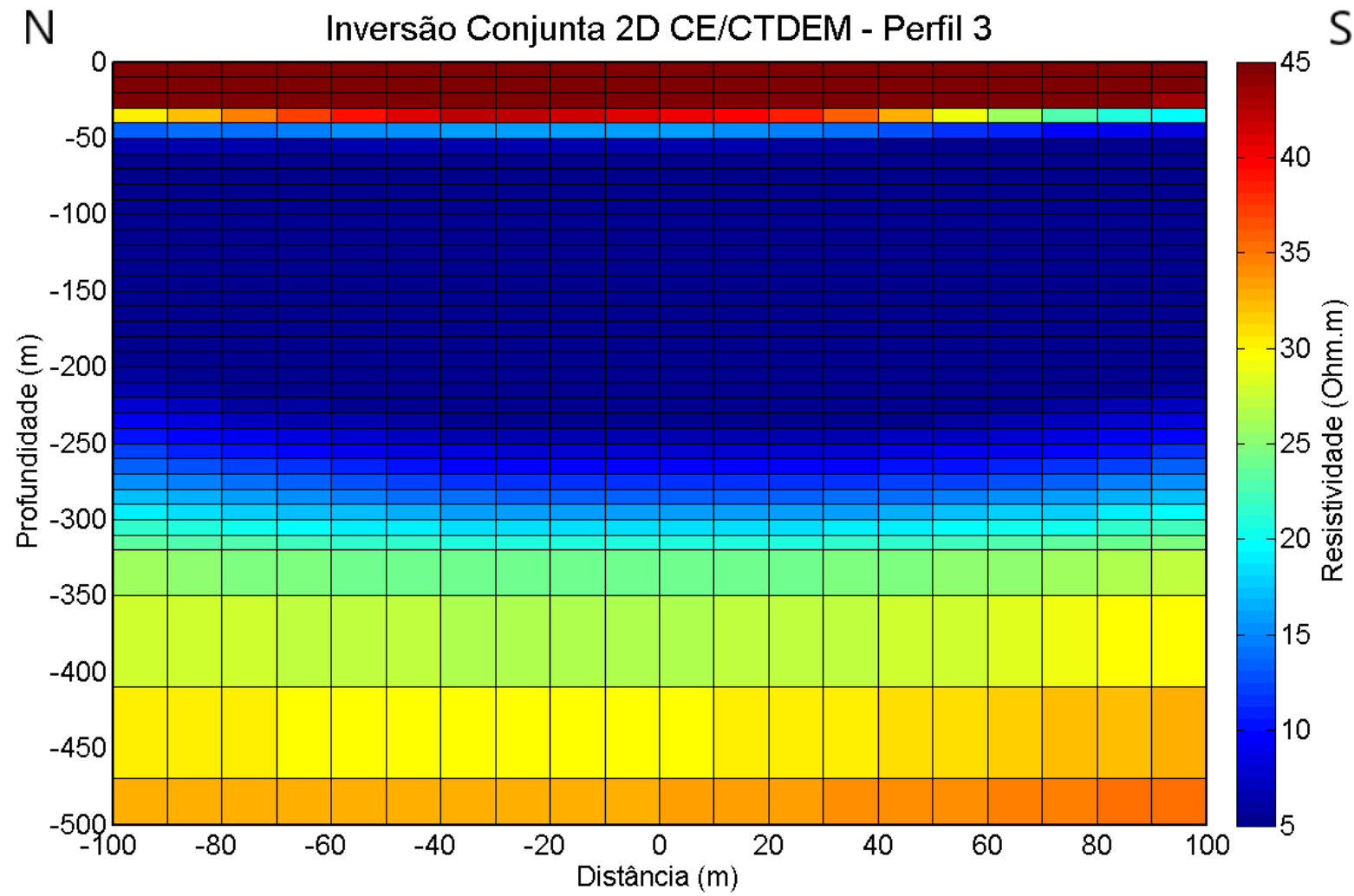

Figura 46 - Resultado da Inversão Conjunta 2D CE/CTDEM para o perfil 3 (N-S) com a escala de cores entre $5 \Omega \cdot m$ e $45 \Omega \cdot m$ 


\subsection{Interpretação dos Resultados}

As Figuras 47, 48 e 49 apresentam às interpretações geológicas dos modelos geoelétricos obtidos a partir das inversões conjuntas dos perfis $1(\mathrm{E}-\mathrm{W}), 2(\mathrm{~N}-\mathrm{S})$ e $3(\mathrm{~N}-\mathrm{S})$, respectivamente.

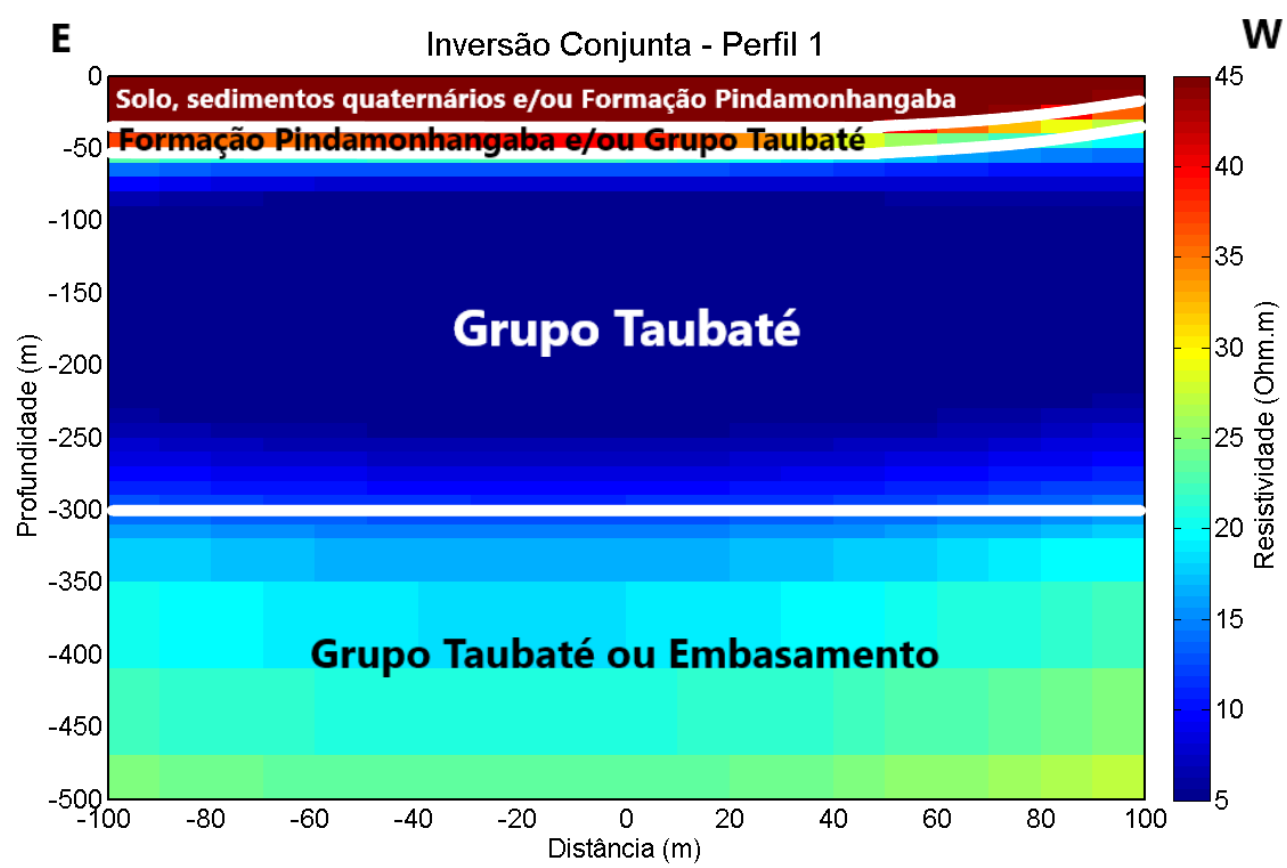

Figura 47 - Interpretação geológica do modelo geoelétrico obtido a partir da Inversão Conjunta para o perfil $1(\mathrm{E}-\mathrm{W})$.

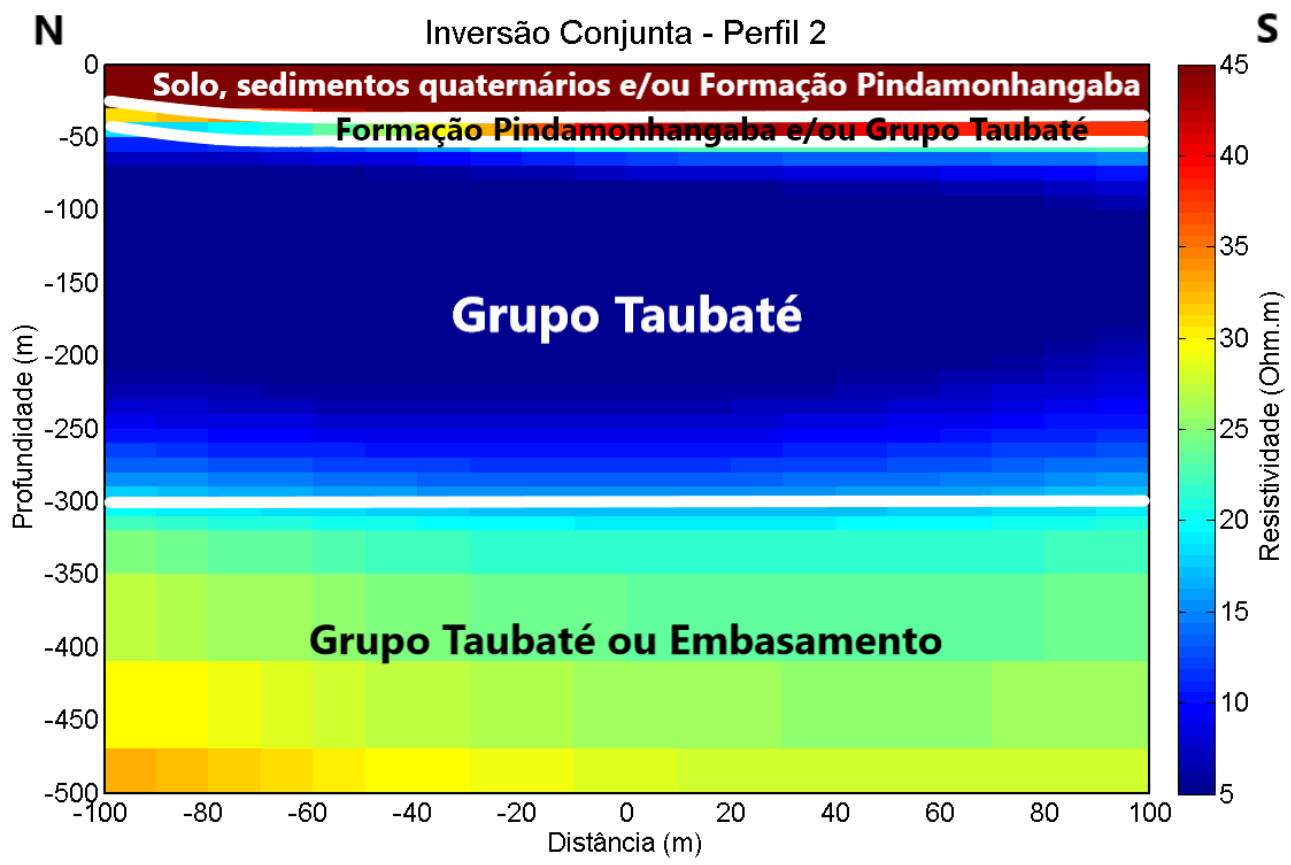

Figura 48 - Interpretação geológica do modelo geoelétrico obtido a partir da Inversão Conjunta para o perfil 2 (N-S). 
A primeira camada até $30 \mathrm{~m}$ de profundidade é resistiva, apresentando resistividade entre $45 \Omega \cdot \mathrm{m}$ e $250 \Omega \cdot \mathrm{m}$. Essa primeira camada representa o solo e pode conter sedimentos quaternários e/ou a Formação Pindamonhangaba, que é terciária. A partir das informações de poços, a camada de solo mais sedimentos quaternários juntos apresentam no máximo $20 \mathrm{~m}$ de espessura. Portanto, não foi possível determinar o contato entre os sedimentos Quaternários e Terciários.

As camadas resistivas mais rasas, até $\sim 30 \mathrm{~m}$ de profundidade, foram melhor determinadas pelos CE's, pois o método TDEM possui baixa resolução para as camadas mais rasas resistivas.

A segunda camada entre $30 \mathrm{~m}$ e $50 \mathrm{~m}$ de profundidade, possui resistividade média de $40 \Omega \cdot \mathrm{m}$, representando sedimentos condutivos intermediários, os quais podem representar a Formação Pindamonhangaba e/ou sedimentos do Grupo Taubaté.

Entre $50 \mathrm{~m}$ e $300 \mathrm{~m}$ de profundidade observa-se uma camada muito condutiva, com valores de resistividade entre $5 \Omega \cdot \mathrm{m}$ e $20 \Omega \cdot \mathrm{m}$, sendo interpretada como rochas sedimentares terciárias do Grupo Taubaté, o qual é constituído principalmente por intercalações de folhelhos, que formam aquicludes, e arenitos, que formam o aquífero. Como tanto os folhelhos quanto os arenitos são muito condutivos, eles foram definidos como uma única camada a partir do método TDEM, pois não há resolução para separá-los.

Em $~ 300 \mathrm{~m}$ de profundidade é possível observar uma interface onde a resistividade aumenta para valores entre $20 \Omega \cdot \mathrm{m}$ e $40 \Omega \cdot \mathrm{m}$. As inversões conjuntas conseguiram definir melhor a profundidade dessa interface, pois nas inversões individuais de CTDEM do perfil 1 (E-W) (Figura 36) e do perfil 2 (N-S) (Figura 40), a mesma encontra-se em $\sim 280 \mathrm{~m}$ de profundidade. A partir dos valores de resistividade, é possível que essa interface represente uma variação faciológica do Grupo Taubaté ou, conforme será discutido a seguir, pode representar o topo do embasamento.

A Figura 50 apresenta um mapa de espessura de sedimentos, que foi elaborado por Cogné et al. (2013) com base no trabalho original de Marques (1990). A localização da área estudo é indicada na Figura 50, onde é possível estimar a espessura de sedimentos em torno de $300 \mathrm{~m}$. Portanto, é possível que a interface em $300 \mathrm{~m}$ de profundidade represente o topo do embasamento, no entanto, mais estudos são necessários. 


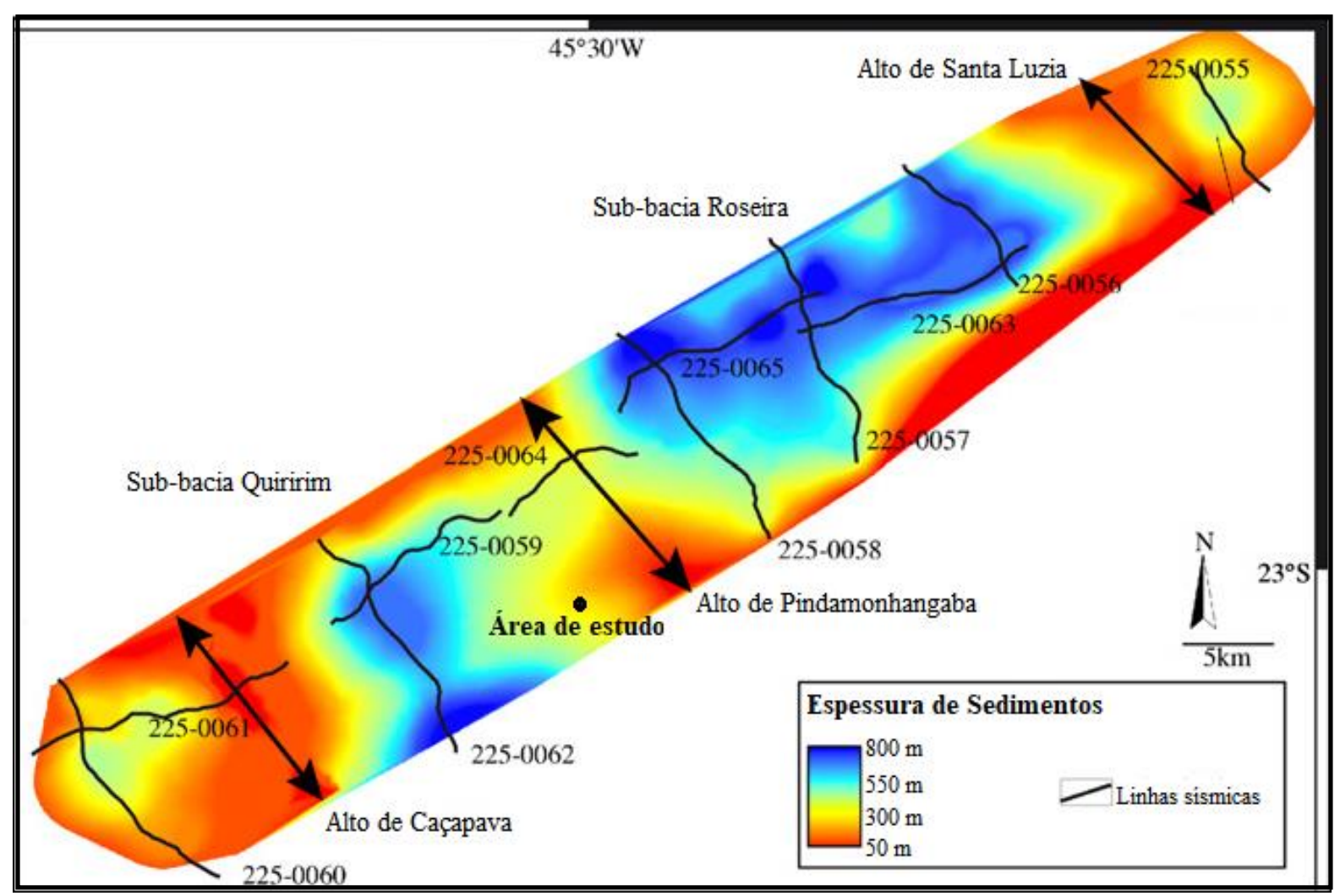

Figura 50 - Mapa de espessura de sedimentos (modificado de Cogné et al., 2013).

O embasamento da Bacia de Taubaté é formado por gnaisses, que é uma rocha muito resistiva, cujo valor de resistividade pode variar entre $68.000 \Omega \cdot \mathrm{m}$ úmida e $3.000 .000 \Omega \cdot \mathrm{m}$ seca (Telford et al., 1990). Conforme foi discutido na seção 3.2.1., o método TDEM é sensível a camadas condutivas por causa da maior indução eletromagnética nessas camadas, quanto mais condutivo o meio, mais lentamente o campo secundário induzido se difunde e vice-versa. Como as rochas do Grupo Taubaté são muito condutivas, o sinal se concentra e se dissipa nessa camada, não sendo capaz de induzir correntes na camada abaixo. Por isso, no caso desta pesquisa, a profundidade de investigação real é de cerca de $300 \mathrm{~m}$, sendo menor que a profundidade de investigação teórica $(1166 \mathrm{~m})$ calculada a partir da equação (38) na seção 3.2.2, pois esta equação é uma aproximação que não considera as características geológicas do meio.

De uma forma geral, os resultados obtidos são coerentes entre si e também com as informações de poços e a geologia da bacia. 


\section{CONCLUSÕES}

A aplicação da metodologia de Inversão Individual e Conjunta de dados de CE e CTDEM apresentou eficiência para a caracterização geoelétrica da Bacia de Taubaté, permitindo o mapeamento do Grupo Taubaté, o qual armazena o Aquífero Taubaté.

As inversões obtiveram erros de ajuste entre $10 \%$ e $20 \%$, e, em geral, resultaram em modelos geoelétricos coerentes com as informações de poços e a geologia da bacia.

As inversões individuais dos CE's permitiram identificar bem as camadas sedimentares mais rasas resistivas e também a camada de sedimentos condutivos intermediários. As inversões individuais dos CTDEM's permitiram identificar a camada condutiva referente ao Grupo Taubaté. Já as inversões conjuntas resultaram em modelos geoelétricos mais completos, apresentando todas as camadas definidas pelos dois métodos geofísicos. Portanto, os resultados mostraram a vantagem da Inversão Conjunta em relação à Inversão Individual, sendo aquela capaz de definir todas as camadas geoelétricas identificadas por ambos os métodos e diminuir as ambiguidades na interpretação dos resultados.

Os resultados não permitiram a determinação do contato entre os sedimentos Quaternários e Terciários, pois a primeira camada resistiva apresentou uma espessura de 30 $\mathrm{m}$, e, a partir das informações de poços, a camada de solo mais sedimentos quaternários apresentam no máximo $20 \mathrm{~m}$ de espessura. Portanto, essa primeira camada resistiva foi interpretada como solo mais sedimentos quaternários e/ou a Formação Pindamonhangaba, a qual é terciária.

O Grupo Taubaté apresentou pelo menos $250 \mathrm{~m}$ de espessura, sendo formado principalmente por folhelhos intercalados por camadas de arenito. Os folhelhos formam aquicludes e os arenitos formam o aquífero, sendo que ambas rochas sedimentares são muito condutivas e, portanto, o método TDEM permitiu identificar como uma única camada geoelétrica.

Como as rochas sedimentares do Grupo Taubaté são muito condutivas e, abaixo, o embasamento é muito resistivo, então, conforme esperado, o método TDEM não permitiu a identificação do embasamento. No entanto, com base na Figura 50 e nas informações de poços, é possível inferir que o topo do embasamento esteja a cerca de $300 \mathrm{~m}$ de profundidade. Porém, mais estudos são necessários, pois, devido a resistividade obtida entre $20 \Omega \cdot \mathrm{m}$ e 40 $\Omega \cdot \mathrm{m}$, existe a possibilidade dessa interface representar uma variação faciológica do Grupo 
Taubaté. Portanto, recomenda-se um levantamento sísmico na área de estudos para se definir o topo do embasamento.

A Bacia de Taubaté possui uma geologia complexa, apresentando sedimentos quaternários e variadas rochas sedimentares terciárias, incluindo arenitos, argilitos, folhelhos, conglomerados e siltitos, portanto, se houvesse um poço com descrição geológica mais próximo da área de estudos, ajudaria na interpretação dos resultados, principalmente em relação as camadas mais rasas, até $50 \mathrm{~m}$ de profundidade.

Estes resultados poderão ser importantes para a cidade de Taubaté, uma vez que devido à recente crise hídrica, o Aquífero Taubaté é uma importante fonte alternativa de água, que poderá ser relevante para o abastecimento doméstico, industrial e/ou agropecuário.

Esta pesquisa contribuiu com o aprimoramento da metodologia de Inversão Conjunta 2D CE/CTDEM. Além disso, contribuiu com o avanço na interpretação dos métodos geoelétricos num contexto geológico da Bacia de Taubaté e corroborou com as pesquisas hidrogeofísicas no Estado de São Paulo. 


\section{REFERÊNCIAS ${ }^{4}$}

Albouy, Y., Andrieux, P., Rakotondrasoa, G., Ritz, M., Descloitres, M., Join, J.L., Rasolomanana, E. Mapping Coastal Aquifers by Joint Inversion of DC and TEM SoundingsThree Case Histories: Ground Water. Vol. 39, no. 1, p. 87-97, 2001.

Almeida, F. F. M. de, Amaral, G., Cordani, U. G., Kawashita, K. The precambrian evolution of the South American Cratonic Margin South of Amazon River. In: A. E. Nairn, F. G. Stehli (eds) The ocean basins and margins. Plenum Publ., New York, N. Y., v. 1, p. 411-446, 1973.

Bortolozo, C. A. Inversão conjunta 1D de dados de SEV e TDEM: aplicações em hidrogeologia. Dissertação (Mestrado em Geofísica), IAG/USP, p. 102, 2011.

Bortolozo, C. A., Couto Jr., M. A., Porsani, J. L., Almeida, E. R., Monteiro Santos, F. A. Geoelectrical characterization using joint inversion of VES/TEM data: A case study in Paraná Basin, São Paulo State, Brazil. Journal of Applied Geophysics 111, p. 33-46, 2014.

Bortolozo, C. A., Porsani, J. L., Santos, F. A. M., Almeida, E. R. VES/TEM 1D joint inversion by using Controlled Random Search (CRS) algorithm. Journal of Applied Geophysics 112, p. 157-174, 2015.

Bortolozo, C. A. Inversão conjunta 1D e 2D de dados de Eletrorresistividade e TDEM aplicados em estudos de hidrogeologia na bacia do Paraná. Tese (Doutorado em Geofísica), IAG/USP, São Paulo, p. 369, 2016.

Bortolozo, C. A., Campaña, J. D. R., Junior, M. A. C., Porsani, J. L. and dos Santos, F. A. M. The Effects of Negative Values of Apparent Resistivity in TEM Surveys. International Journal of Geosciences 7, p. 1182-1190, 2016.

Broyden, C. G. A Class of Methods for Solving Nonlinear Simultaneous Equations. American Mathematical Society. Mathematics of Computation 19, p. 577-593, 1965.

Campaña, J. D. R. Análise do campo magnético secundário 3D usado no método TDEM: Aplicações em aquíferos fraturados. Dissertação (Mestrado em Geofísica), IAG/USP, p. 158, 2015.

Campaña, J. D. R., Porsani, J. L., Bortolozo, C. A., Oliveira, G. S. and Santos, F. A. M. Inversion of TEM Data and Analysis of the 2D Induced Magnetic Field Applied to the Aquifers Characterization in the Paraná Basin, Brazil. Journal of Applied Geophysics 138, p. 233-244, 2017.

Christiansen, A. V., Auken, E., Sørensen, K. The transient electromagnetic method, in R. Kirsch ed., Groundwater geophysics: a tool for hydrogeology, Cap. 6. Springer, Second edition, p. 179-226, 2006.

Cogné, N., Coboold, P.R., Riccomini, C., Gallagher, K. Tectonic setting of the Taubaté Basin (southeastern Brazil): insights from regional seismic profiles and outcrop data. Journal of South American Earth Sciences, 42, p. 194-204, 2013.

\footnotetext{
${ }^{4}$ De acordo com a Associação Brasileira de Normas Técnicas (ABNT NBR 6023).
} 
Couto Jr., M. A. Caracterização hidrogeoelétrica na região de Termas de Ibirá, Bacia do Paraná, por meio de inversão conjunta 1D de sondagens TDEM/SEVs. Dissertação (Mestrado em Geofísica), IAG/USP, p. 148, 2015.

CPRM - Companhia de Pesquisa de Recursos Minerais. SIAGAS - Sistema de Informações de Águas Subterrâneas. Disponível em: <http://siagasweb.cprm.gov.br/layout/>. Acesso em: 31 de janeiro de 2018.

DAEE - Departamento de Águas e Energia Elétrica. Estudo de Águas Subterrâneas - Região Administrativa 3 - São José dos Campos e Faixa Litorânea. São Paulo, p. 175, 1977.

DAEE - Departamento de Águas e Energia Elétrica, IG - Instituto Geológico, IPT - Instituto de Pesquisas Tecnológicas, CPRM - Serviço Geológico do Brasil. Mapa de águas subterrâneas do Estado de São Paulo. São Paulo, p. 119, 2005.

Dey, A., Morrison, F. Resistivity modeling for arbitrarily shaped two-dimensional structures. Geophysical Prospecting 27, p. 106-136, 1979.

DuFort, E. C. e Frankel, S. P. Stability Conditions in the Numerical Treatment of Parabolic Differential Equations. Mathematical Tables and Other Aids to Computation 7, p. 135-152, 1953.

Goldman, M., Du Plooy, A., Eckard. On reducing ambiguity in the interpretation of transient electromagnetic sounding data. Geophysical Prospecting 42, p. 3-25, 1994.

IBGE - Instituto Brasileiro de Geografia e Estatística. Disponível em:

<https://cidades.ibge.gov.br/brasil/sp/taubate/panorama>. Acesso em: 29 de março de 2018.

Kearey, P., Brooks, M., \& Hill, I. An Introduction to Geophysical Exploration. Third Edition. England: Blackwell Science Ltd, 2002.

Leite, D. N. Caracterização geoelétrica por meio de inversão conjunta 1D de SEV/TDEM na região de Urupês-SP, Bacia do Paraná: aplicações em hidrogeologia. Dissertação (Mestrado em Geofísica), IAG-USP, p. 95, 2013.

Leite, D.N., Bortolozo, C.A., Porsani, J.L., Couto Jr., M.A., Campaña, J.D.R., Monteiro Santos, F.A., Rangel, R.C., Hamada, L.R., Sifontes, R.V., Oliveira, G.S., Stangari, M.C. Geoelectrical Characterization with 1D VES/TDEM Joint Inversion in Urupês-SP Region, Paraná Basin: Applications to Hydrogeology. Journal of Applied Geophysics, 2018. Accepted.

Marques, A. Evolução tectono-sedimentar e perspectivas exploratórias da Bacia de Taubaté. São Paulo, Brasil. Boletim de Geociências da Petrobrás 4, p. 253-262, 1990.

McNeill, J. D. Principles and application of time domain electromagnetic techniques for resistivity sounding, Technical Note TN-27, Geonics Ltd, Mississauga, Ontario, Canada, 1994.

Meju, M. A. Joint inversion of TEM and distorted MT soundings: some effective practical considerations. Geophysics 61 (1), p. 56-65, 1996. 
Oristaglio, M. L. e Hohmann, G. W. Diffusion of electromagnetic fields into a twodimensional earth: A finite-difference approach. Geophysics 49, p. 870-894, 1984.

Porsani, J. L., Almeida, E. R., Bortolozo, C. A., Monteiro Santos, F. A. TDEM survey in an area of seismicity induced by water wells in Paraná sedimentary basin, Northern São Paulo State, Brazil. Journal of Applied Geophysics 80, p. 1-9, 2012a.

Porsani, J. L., Bortolozo, C.A., Almeida, E. R., Santos Sobrinho, E. N., Santos, T. G. TDEM survey in urban environmental for hydrogeological study at USP campus in São Paulo city, Brazil. Journal of Applied Geophysics 76, p. 102-108, 2012 b.

Raiche, A. P., Jupp, D. L. B., Rutter, H., Vozoff, K. The joint use of coincident loop transient electromagnetic and Schlumberger sounding to resolve layered structures. Geophysics 50, p. 1618-1627, 1985.

Riccomini, C. O Rift Continental do Sudeste do Brasil. Tese (Doutorado em Geologia), IGc/USP, p. 256, 1989.

Riccomini, C., Sant'anna, L. G., Ferrari, A. L. Evolução Geológica do Rift Continental do Sudeste do Brasil. In: Manteso-Neto, V., Bartorelli, A., Carneiro, C. D. R., Neves, B. B. B. Geologia do Continente Sul-Americano: Evolução da Obra de Fernando Marques de Almeida. Beca, São Paulo, p. 384-405, 2004.

Sasaki, Y. Two-dimensional joint inversion of magnetotelluric and dipole-dipole resistivity data. Geophysics 54, p. 254-262, 1989.

Sørensen, K. I., Auken, E., Thomsen, P. TDEM in groundwater mapping - a continuous approach. Proceedings of the Symposium on the Application of Geophysics to Engineering and Environmental Problems, Arlington, Virginia, p. 485 - 491, 2000.

Souza Filho, M. N., Pereira, S. Y., Barbuena, D. Potencial geotérmico da Bacia sedimentar de Taubaté baseado em dados de perfilagem geofísica em poços. Águas Subterrâneas 27 (1), p. 37-52, 2013.

Spies, B. R. Depth of Investigation in electromagnetic sounding methods: Geophysics, 54, p. 872-888, 1989.

Telford, W. M., Geldart, L. P. Sheriff, R. E. Applied Geophysics. Second Edition. Cambridge University Press, 1990.

Vidal, A.C., Fernandes, F.L., Chang, H.K. Distribuição dos arenitos na bacia de Taubaté - SP. São Paulo, UNESP. Geociências 23, p. 55-66, 2004.

Vozoff, K., Jupp, D. L. B. Joint inversion of geophysical data. Geophysical Journal International 42 (3), p. 977-991, 1975.

Yang, C. H., Tong, L. T. A study of joint inversion of direct current resistivity, transient electromagnetic and magnetotelluric sounding data. Terrestrial, Atmospheric and Oceanic Sciences (TAO) 10 (1), p. 293-301, 1999. 


\section{ANEXOS}

9.1 Resumo expandido para $01^{\circ}$ Congresso Internacional da Sociedade Brasileira de Geofísica 


\section{Geoelectrical analysis from electroresistivity and TDEM data applied to geological and hydrogeological studies in Taubaté Basin: Preliminary results}

Rodrigo Corrêa Rangel (USP/IAG) ${ }^{*}$, Jorge Luís Porsani (USP/IAG), Cassiano Antônio Bortolozo (USP/IAG), Luiz Rodrigo Hamada (USP/IAG), Gabriela Serejo de Oliveira (USP/IAG), Marcelo Cesar Stangari (USP/IAG), Ernande Costa Santos (USP/IAG)

Copyright 2017, SBGf - Sociedade Brasileira de Geofísica

This paper was prepared for presentation during the $15^{\text {th }}$ International Congress of the Brazilian Geophysical Society held in Rio de Janeiro, Brazil, 31 July to 3 August, 2017.

Contents of this paper were reviewed by the Technical Committee of the $15^{\text {th }}$ International Congress of the Brazilian Geophysical Society and do not necessarily represent any position of the SBGf, its officers or members. Electronic reproduction or storage of any part of this paper for commercial purposes without the written consent of the Brazilian Geophysical Society is prohibited.

\section{Abstract}

In this research, we have performed a geoelectrical analysis from electroresistivity (ER) and time domain electromagnetic (TDEM) data to study the geology and hydrogeology in Taubaté city, that is on the Taubaté Sedimentary Basin, which is characterized by halfgrabens. ER method is more accurate for resistive structures and presents good resolution for shallower layers, on the other hand, TDEM method is more accurate for conductive structures and can reach great investigation depth, therefore these methods are complementary to each other. The preliminary results permit to define the top of the shallow sedimentary aquifer and the contact of the Quaternary and Tertiary sediments. The next step will be to perform a 2D TDEM/ER joint inversion using a new methodology developed by Bortolozo (2016), which uses the advantage of both methods to minimize the ambiguities and characterize 2D structures with more accuracy.

\section{Introduction}

In geophysics applied to natural resources exploration, the ambiguities in the results interpretation are always present. One way to reduce such ambiguities is to jointly use more than one geophysical method. With a wider range of data, the ambiguities can be reduced and the final interpretation becomes more reliable. The problem consists of looking for physical properties information of the subsurface. For example, the electrical resistivity can be obtained through electrical and electromagnetic methods.

In the ER method, the electrical resistivity is obtained by injecting an electric current in the subsurface through two electrodes ( $A$ and $B$ ) and the potential is measured by another two electrodes ( $\mathrm{M}$ and $\mathrm{N}$ ). For TDEM method, the resistivity is obtained as a function of the time response of the medium, i.e., the transient magnetic field.

The study area is located in Taubaté basin, next to Taubaté city, São Paulo State, Brazil (Figure 1). According to Riccomini et al. (2004) Taubaté basin is the largest basin of the Continental Rift of Southeastern Brazil
(CRSB) with $170 \mathrm{~km}$ length and $20 \mathrm{~km}$ width, covering an area of about $3200 \mathrm{~km}^{2}$. Figure 2 shows a geologic map of Taubaté Basin. The basin is elongated in NE-SW direction and presents normal faults in NW-SE direction, it presents a rift architecture and is characterized by a series of half-grabens with $\sim 850 \mathrm{~m}$ thickness of sediments.

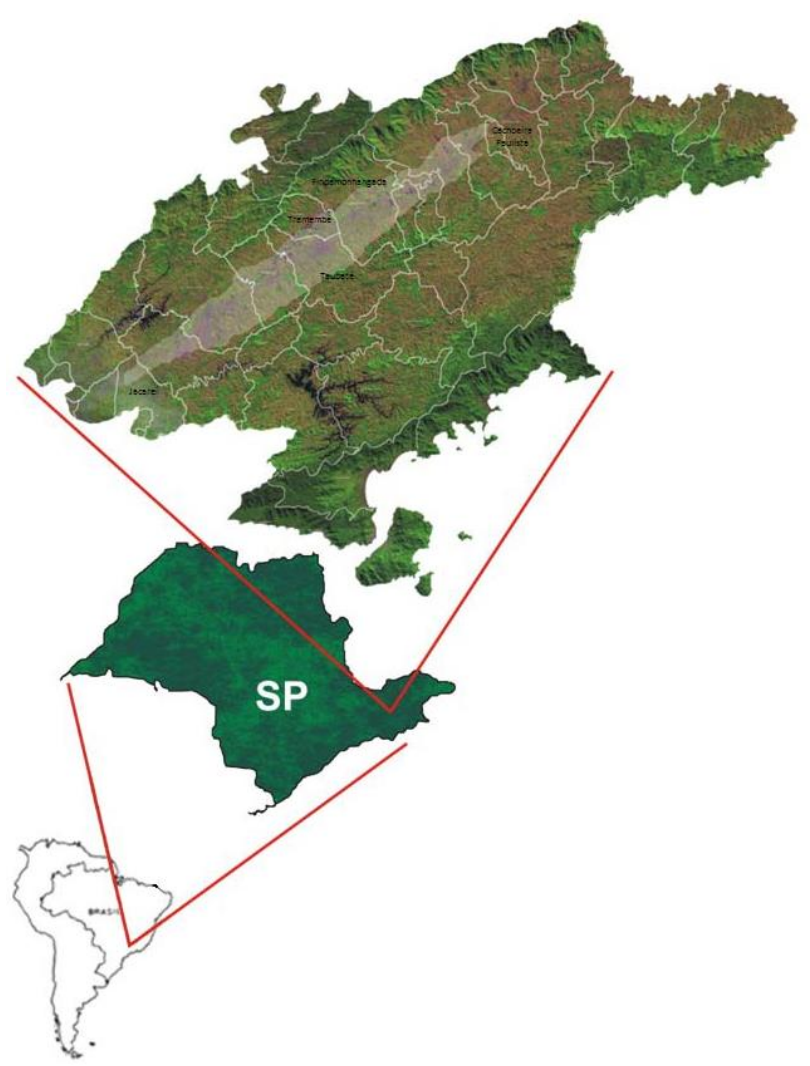

Figure 1. Taubaté Basin, São Paulo State, Brazil (Ribeiro, 2010).

The study aims to characterize the geoelectrical stratigraphy of the subsurface to locate the contact of the Quaternary and Tertiary sediments, to map the shallow sedimentary aquifer, to define the top of the basement rocks and to determine the fault zones that form the halfgrabens. 


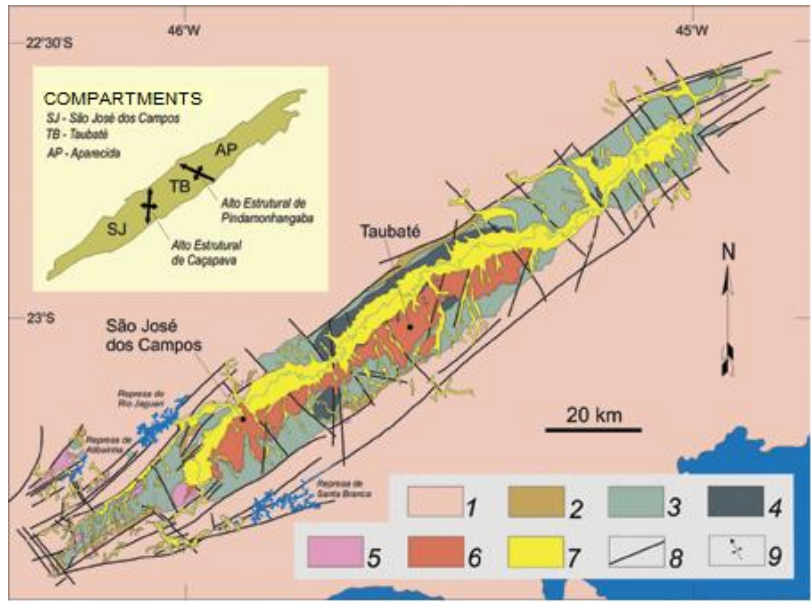

Figure 2. Geologic map of the Taubaté Basin: 1) Precambrian basement rocks; 2) Resende Formation; 3) Resende Formation; 4) Tremembé Formation; 5) São Paulo Formation; 6) Pindamonhangaba Formation; 7) Quaternary sediments; 8) Cenozoic faults, in part reactivated from Precambrian basement shear zones; 9) main fold axes (adapted from Riccomini et al., 2004).

\section{Method}

\section{Electroresistivity}

ER is a geophysical method widely used in the investigation of mineral deposits, groundwater exploration, evaluation of contaminated areas, among others (Telford et al., 1990). The method consists in injecting electric current (I) into the ground through metallic electrodes ( $A B$ current electrodes), and measuring the potential $(\Delta V)$ by another two electrodes (MN potential electrodes). Electrical resistivity values can be estimated because the spatial arrangement of the electrodes is known. According to Koefoed (1979), the apparent electrical resistivity $\left(\rho_{a}\right)$ of the subsurface can be expressed as:

$$
\rho_{\mathrm{a}}=\mathrm{k} \frac{\Delta \mathrm{V}}{\mathrm{I}}
$$

where $\mathrm{k}$ is the geometric factor, which depends on the spacing and arrangement of the electrodes chosen for the survey. The investigation depth depends on the medium and the separation of the electrodes. Greater depths are achieved when the array size is increased.

Increasing centered arrangements on the same point constitutes the investigation procedure called Vertical Electrical Sounding (VES), which investigates the vertical variation of the resistivity (1D investigation). On the other hand, the electrical resistivity imaging (Figure 3 ) allows to map the lateral variation of the resistivity (2D investigation), used in this study.

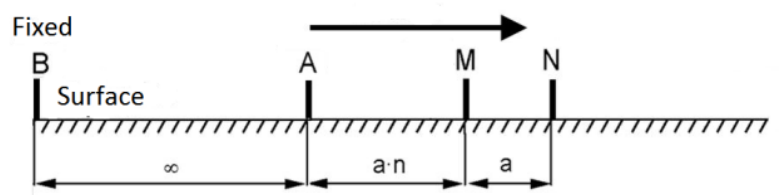

Figure 3. Electrical resistivity imaging, pole-dipole array (adapted from Bortolozo, 2016).

\section{Time Domain Electromagnetic Method}

The TDEM method investigates the lateral and vertical variations of the electrical resistivity of rocks in subsurface through the induction of electric currents in the interior of the Earth, being based on the principle of electromagnetic induction (Christiansen et al., 2006). It consists of having a square transmitter loop and a receiver coil, both on the surface. Initially a continuous current is injected in the transmitter loop, then this current is switched off and in the interval, in the order of microseconds, that the current goes to zero, the primary electromagnetic field varies in time and induces a secondary electromagnetic field, also variable in time, in the subsurface materials. The variation of the secondary magnetic field is measured by the receiver coil.

According to McNeill (1994), the apparent resistivity in late times is expressed by the equation:

$$
\rho_{\mathrm{a}}(\mathrm{t})=\frac{\mathrm{k}_{1} \mathrm{M}^{2 / 3}}{\mathrm{~V}(\mathrm{t})^{2 / 3} \mathrm{t}^{5 / 3}}
$$

where $\rho_{a}(t)$ is the apparent electrical resistivity (Ohm.m), $\mathrm{k}_{1}=\mu_{0}{ }^{5 / 3} \mathrm{M} / 20 \pi\left(\mu_{0}=4 \pi \times 10^{-7} \mathrm{H} / \mathrm{m}\right.$, which is the vacuum magnetic permeability), $M$ is the magnet moment that is equals to the product of the current $(A)$ by the area $\left(\mathrm{m}^{2}\right)$, $\mathrm{V}(\mathrm{t})$ is the normalized voltage measured by a unit coil with $1 \mathrm{~m}^{2}$ area and $\mathrm{t}$ is the interval given in seconds.

The voltage $\mathrm{V}(\mathrm{t})$ variation as a function of time is associated with the electrical resistivity variation of the subsurface materials.

Some of the main applications of the TDEM method include applications in groundwater prospecting in sedimentary aquifers (McNeill, 1994; Jens et al., 2003; Carrasquilla \& Ulugergerli, 2006; Porsani et al., 2012a, $2012 b$ ), aquifers in fractured crystalline rocks (Porsani et al., 2012b), mineral exploration (McNeill, 1994) and contamination of the environment.

There are many acquisition arrays and field procedures. The array configuration, size and other parameters depends on the purpose of the research. The TDEM method can be used in different environments, terrestrial, marine or airborne, making it quite versatile for different situations.

The central loop array (Figure 4) has the advantage of having a good signal-to-noise ratio. In this configuration, the receiver coil is placed in the center of the transmitter loop. With the fixed transmitter loop array and the receiver coil mobile, it is possible to investigate the subsurface structures along the centerline of the transmitter loop. This array also has the advantage of being easy to 
implement and thus allows time saving during data acquisition.

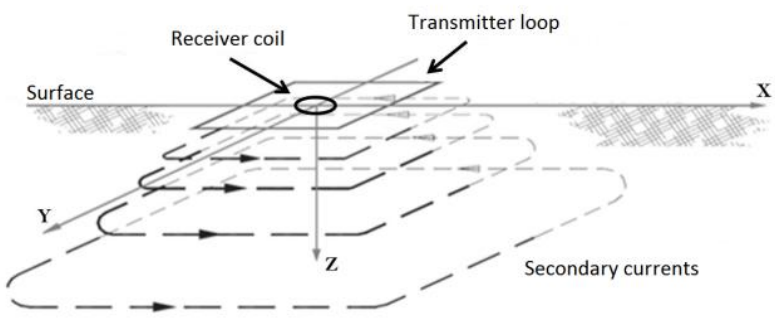

Figure 4. TDEM central loop array showing the secondary currents induced in the subsurface (adapted from McNeil, 1994).

\section{Data Acquisition}

ER data was acquired with the equipment Syscal (Iris Instruments) and it was done with the electrical imaging technique with pole-dipole array (Figure 3 ). The imaging line was $400 \mathrm{~m}$ length (Figure 5) and two profiles were done with $10 \mathrm{~m}$ and $20 \mathrm{~m}$ of electrodes spacing.

TDEM data were acquired with the equipment PROTEM57MK2 (Geonics, 1998), which consist of a transmitter that is connected to a current generator to produce the primary electromagnetic field, and a $3 D$ receiver coil of about $1 \mathrm{~m}$ diameter that is connected to a computer to record the signal of the secondary electromagnetic field induced in the subsurface. The transmitter loop was $200 \times 200 \mathrm{~m}$ and the receiver coil were moved in the centerline of the array within $25 \mathrm{~m}$ spacing, in a total of 7 soundings points (Figure 5 ).

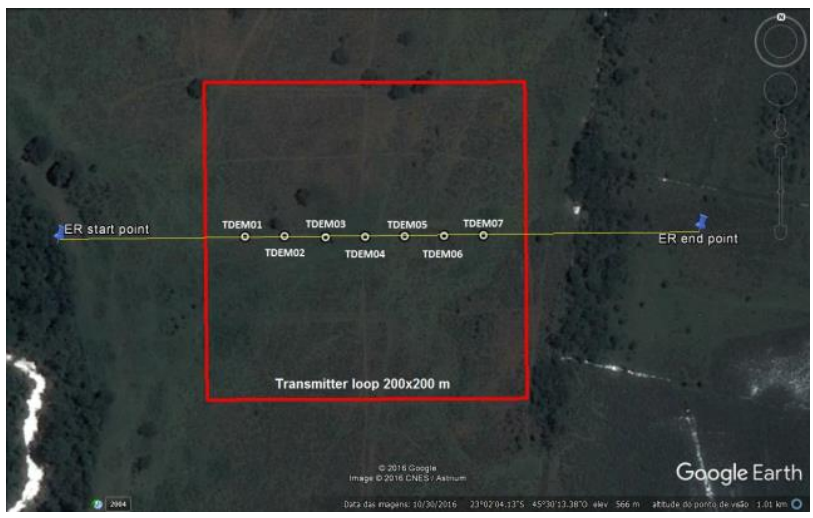

Figure 5. Data acquisition points in Taubaté city. The blue points show the starting and ending points of the ER imaging with $400 \mathrm{~m}$ length. The red square is the TDEM transmitter loop with $200 \times 200 \mathrm{~m}$ and the points inside de loop (TDEM01, TDEM02, ... TDEM07) are the TDEM acquisition points with $25 \mathrm{~m}$ spacing.

\section{Well Information}

Figure 6 shows a map with 40 wells (blue points) drilled in Taubaté and the study area (red square). These well information are available in the Groundwater Information System of the Brazilian Geological Service (http://siagasweb.cprm.gov.br/layout/index.php).

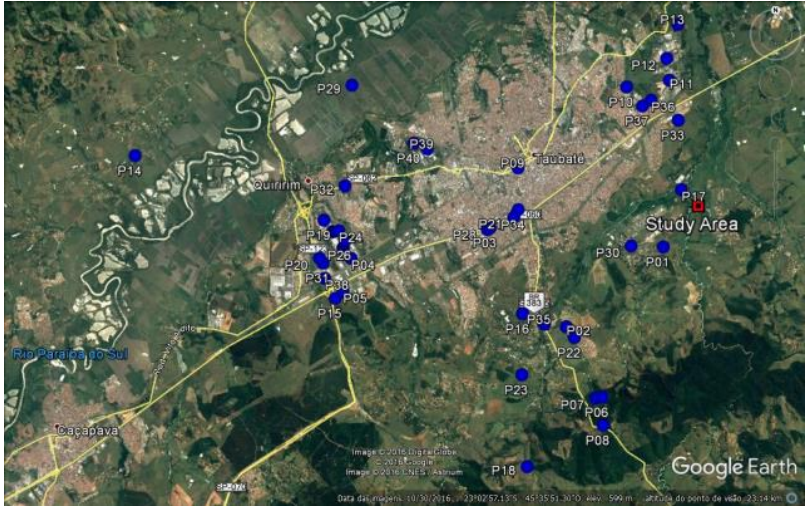

Figure 6. Map showing the wells (40) in blue and the study area in red.

The information include also a geological profile for each well. The closest well is P17, distant around $750 \mathrm{~m}$ from the study area, but its geological profile starts at $78 \mathrm{~m}$ depth and goes to $115 \mathrm{~m}$ depth. P01 is around $1780 \mathrm{~m}$ distant and has $124 \mathrm{~m}$ depth. The deepest well is P40, with $647 \mathrm{~m}$ depth, Table 1 shows its geological profile. P40 is around 9,5 km distant and it is in the same altitude as the study area, $567 \mathrm{~m}$. We can observe that the geology is complex, with variegated shales, sandstones with different grain sizes, shale with gneissic pebbles bellow $400 \mathrm{~m}$ depth and the basement bellow $510 \mathrm{~m}$ depth is formed by gneisses.

Table 2 shows 9 wells with the water level information available from SIAGAS-CPRM. Note that the static water level varies from 0 to around $40 \mathrm{~m}$ depth. For wells P01 to P05 and P09, the lithology is mainly formed by claystone and sandstone and the static water level varies from around 7 to $42 \mathrm{~m}$. For wells P06 to P08, the gneissic basement is shallower, around $10 \mathrm{~m}$ depth and the static water level is shallower.

All well information are very important for the results interpretation.

Table 1. Geological profile of well P40.

\begin{tabular}{|c|c|c|}
\hline From $(\mathbf{m})$ & To $(\mathbf{m})$ & Lithology \\
\hline 0 & 4 & Clayey soil \\
\hline 4 & 11 & Variegated clays \\
\hline 11 & 16 & Conglomeratic sandstone \\
\hline 16 & 401 & Green shale \\
\hline 401 & 485 & Shale with gneissic pebbles \\
\hline 485 & 493 & Sandy shale \\
\hline 493 & 510 & Shale with gneissic pebbles \\
\hline 510 & 650 & Gneisses \\
\hline
\end{tabular}


Table 2. Wells with water level information.

\begin{tabular}{|c|c|c|c|c|c|}
\hline Well & $\begin{array}{c}\text { Depth } \\
(\mathbf{m})\end{array}$ & $\begin{array}{c}\text { Distance }^{1} \\
(\mathbf{k m})\end{array}$ & $\begin{array}{c}\text { Quota }^{2} \\
(\mathbf{m})\end{array}$ & $\begin{array}{c}\mathbf{S L}^{3} \\
(\mathbf{m})\end{array}$ & $\begin{array}{c}\mathbf{D L}^{4} \\
(\mathbf{m})\end{array}$ \\
\hline P01 & 124 & 1.78 & 55 & 42 & 56 \\
\hline P02 & 186 & 6.30 & 27 & 17.6 & 75.47 \\
\hline P03 & 122.5 & 6.84 & 39 & 20.05 & 95.6 \\
\hline P04 & 120 & 11.47 & 22 & 18 & 110 \\
\hline P05 & 270 & 11.92 & 33 & 34.6 & 195 \\
\hline P06 & 80 & 7.00 & 90 & 2 & 56 \\
\hline P07 & 100 & 6.84 & 87 & 0 & 70 \\
\hline P08 & 156 & 7.67 & 121 & 0.2 & 33.5 \\
\hline P09 & 95 & 6.02 & 15 & 7.2 & 65.78 \\
\hline
\end{tabular}

1. Distance from the study area

2. Altitude difference from the study area (all higher)

3. Static water level (before pumping)

4. Dynamic water level (after pumping)

\section{Results}

The electrical resistivity imaging data were inverted with the RES2DINV software (Loke 2004a), which uses the field data to automatically determine a two-dimensional model of resistivity for the medium. Figures 7 and 8 show the results for the array with $10 \mathrm{~m}$ and $20 \mathrm{~m}$ spacing, respectively. Both figures show: the field data (a), the synthetic pseudo-section (b), and the resulting inverted model (c). Analyzing both results, the resistivity sections (c) show three main zones. The first zone, from 0 to around $20 \mathrm{~m}$ depth, the resistivity varies from around 200 Ohm.m to 100 Ohm.m, which can be interpreted as clayey soil and unconsolidated sedimentary rocks. The second zone, from $20 \mathrm{~m}$ to around $40 \mathrm{~m}$ depth, the resistivity varies from around $100 \mathrm{Ohm} . \mathrm{m}$ to $20 \mathrm{Ohm} . \mathrm{m}$, which can be interpreted as claystones and sandstones. The third zone bellow $40 \mathrm{~m}$ depth, with resistivity values lower than 15 Ohm.m, i.e., a conductive zone, can be characterized as rocks filled with water. This saturated zone possibly represents the top of shallow sedimentary aquifer.

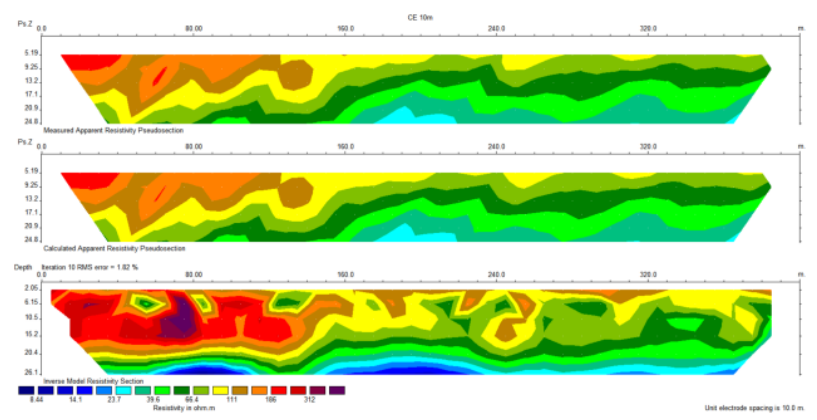

Figure 7. Resistivity section for the $10 \mathrm{~m}$ electrodes spacing.

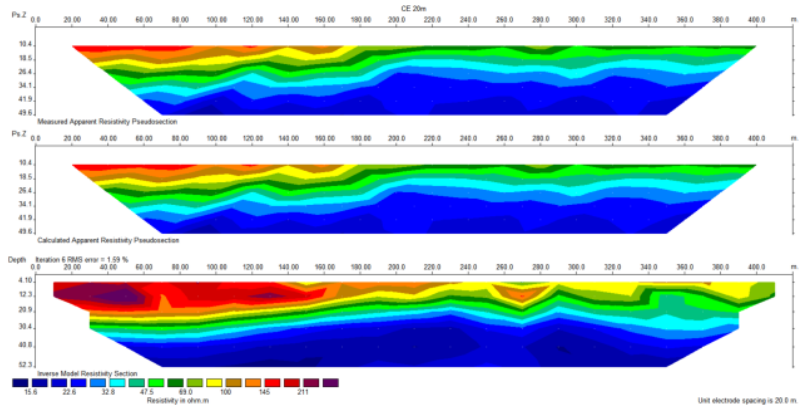

Figure 8. Resistivity section for the $20 \mathrm{~m}$ electrodes spacing.

The TDEM soundings were processed with the IX1D software (Interpex Limited). The inversion process consists of determine the electrical resistivity and thickness of the subsurface layers from the data. Figure 9 shows the inversion result for the central loop sounding TDEM04. In the left side is the apparent resistivity (Ohm.m) versus time ( $\mathrm{ms}$ ) and, in the right side, the geoelectrical model, depth (m) versus resistivity (Ohm.m). It was possible to interpret 6 geoelectrical layers up to $500 \mathrm{~m}$ depth. We limited the depth in $500 \mathrm{~m}$ because bellow that the method loses resolution.
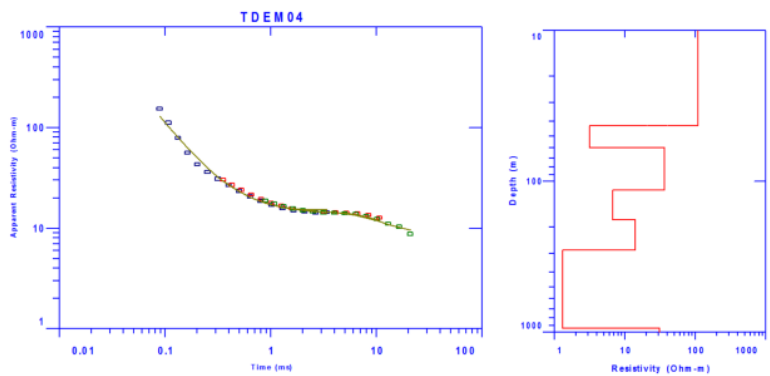

Figure 9. Inversion result for the central loop sounding TDEM04.

The first layer up to $40 \mathrm{~m}$ is consistent to the ER result, resistive with around $100 \mathrm{Ohm} . \mathrm{m}$, which represent the clayey soil, claystone and sandstone. The second layer is also consistent with the ER result, from $40 \mathrm{~m}$ to $60 \mathrm{~m}$ depth, conductive with around $3 \mathrm{Ohm} . \mathrm{m}$, which possibly represent the shallow aquifer. The third layer, from $60 \mathrm{~m}$ to around $110 \mathrm{~m}$ is moderately resistive, around 30 Ohm.m, which can represent a drier sedimentary rock, maybe shale, that has lower porosity and permeability. The forth layer from around $110 \mathrm{~m}$ to $200 \mathrm{~m}$ is conductive, around $8 \mathrm{Ohm} . \mathrm{m}$, can be a saturated rock, probably with higher sand content, presenting higher porosity and permeability. The fifth layer from around $200 \mathrm{~m}$ to $300 \mathrm{~m}$ is still conductive, with the resistivity a little bit higher than the forth, around 12 Ohm.m. Finally, the sixth layer bellow $300 \mathrm{~m}$ is very conductive, around $1 \mathrm{Ohm} . \mathrm{m}$, it is in the limit of the TDEM method resolution.

From the second to the fifth layers can be interpreted as the Tertiary sediments of Taubaté Group, a conductive zone with the resistivity varying from $3 \mathrm{Ohm} . \mathrm{m}$ to 30 
Ohm.m, where the second layer possibly represent the shallow aquifer and it is consistent with the water level from the wells information (Table 2). Taubaté Group presents a very variable lithology and the resistivity can vary a lot too. More resistive zones can have lower porosity and permeability, probably shales, and more conductive zones can represent saturated rocks with a higher sand content and, consequently, higher porosity and permeability.

To summarize, based on the Taubaté Basin geology and the well information, combining ER and TDEM results, we have interpreted three main zones, that are presented in Table 3.

Table 3. Results Interpretation summary.

\begin{tabular}{|c|c|c|c|c|}
\hline Zones & $\begin{array}{c}\boldsymbol{\rho} \\
\text { (Ohm.m) }\end{array}$ & $\begin{array}{c}\text { Depth } \\
\mathbf{( m )}\end{array}$ & Age & Interpretation \\
\hline $1^{\text {st }}$ & $200-100$ & $0-20$ & Quaternary & $\begin{array}{c}\text { Soil and } \\
\text { unconsolidated } \\
\text { rocks }\end{array}$ \\
\hline $2^{\text {nd }}$ & $30-3$ & $20-$ & Tertiary & $\begin{array}{c}\text { Conglomerate, } \\
\text { sandstone, } \\
\text { siltstone, } \\
\text { claystone and } \\
\text { shale (Taubaté } \\
\text { Group) }\end{array}$ \\
\hline $3^{\text {rd }}$ & $<15$ & 400 & Tertiary & $\begin{array}{c}\text { Top of shallow } \\
\text { sedimentary } \\
\text { aquifer }\end{array}$ \\
\hline
\end{tabular}

\section{Conclusions}

The results have shown a great potential for the application of the combined ER and TDEM methods to the geoelectrical characterization of the Taubaté Basin, making it possible to identify the contact between Quaternary and Tertiary sediments and the top of the shallow aquifer. The results are consistent with the Taubaté Basin geology described in the literature and with the well information.

With the 2D TDEM/ER joint inversion, that is the next step in the project, we expect to define 2D structures, such as the half-graben faults. We will also have ER and TDEM data in N-S direction and seismic data to collaborate the interpretation.

\section{Acknowledgments}

RCR thanks to CAPES for providing the scholarship. JLP thanks to FAPESP (2009/08466-3 and 2012/15338-4), CNPq-PVE (406653/2013-5) for the financial support to develop this research and CNPq (301692/2013-0) for research grant. CAB thanks to FAPESP for the scholarship (2011/06404-0) and CNPq for the Postdoctoral scholarship (403389/2015-1). Thanks to the University of Sao Paulo for providing the infrastructure support and to the colleagues Luiz and Gabriela for sharing their knowledge, Ernande and Marcelo for the data acquisition.

\section{References}

Bortolozo, C.A., 2016. Inversão conjunta 1D e 2D de dados de Eletrorresistividade e TDEM aplicados em estudos de hidrogeologia na bacia do Paraná. Tese de Doutorado (Geofísica), IAG-USP, São Paulo, 369 p.

Carrasquila, A.A.G.; Ulugergerli, E., 2006. Evaluation of the transient electromagnetic geophysical method for stratigraphic mapping and hydrogeological delineation in campos basin, Brazil. Revista Brasileira de Geofísica 24, 333-341.

Christiansen, A.V.; Auken, E.; Sørensen, K., 2006, The transient electromagnetic method, in R. Kirsch ed., Groundwater geophysics a tool for hydrogeology, Cap.6, 179-225.

Geonics, 1998. PROTEM-57MK2, operating manual. Geonics Limited, Mississauga, Ontario, Canada.

Jens E. D.; Auken, E.; Jørgensen F.; Søndergaard, V.; Sørensen, K.I., 2003. The application of the transient electromagnetic method in hydrogeophysical surveys. Journal Applied Geophysics 53, 181-198.

Koefoed, O., 1979. Geosounding principles 1: Resistivity sounding measurements, Elsevier, Chapter II.

Loke M. H., 2004a. Res2Dinv versão 3.54 for Windows 98/Me/2000/NT/XP. Rapid 2D Resistivity and IP Inversion using the least-squares method. Software Manual. $133 \mathrm{p}$.

McNeill, J.D., 1994. Principles and application of time domain electromagnetic techniques for resistivity sounding, Technical Note TN-27, Geonics Ltd, Mississauga, Ontario, Canada.

Porsani, J.L., Almeida, E.R., Bortolozo, C.A., Monteiro Santos, F.A., 2012a. TDEM survey in an area of seismicity induced by water wells in Paraná sedimentary basin, Northern São Paulo State, Brazil. Journal of Applied Geophysics 80, 1-9.

Porsani, J.L., Bortolozo, C.A., Almeida, E.R., Santos Sobrinho, E. N., Santos, T.G., 2012b. TDEM survey in urban environmental for hydrogeological study at USP campus in São Paulo city, Brazil. Journal of Applied Geophysics 76, 102-108.

Ribeiro, G.C., 2010. Avaliação morfológica, taxonômica e cronológica dos mamíferos fósseis da Formação Tremembé (Bacia de Taubaté), Estado de São Paulo, Brasil. Dissertação de Mestrado (Zoologia), IB-USP, São Paulo, $112 \mathrm{p}$.

Riccomini, C.; Sant'anna, L. G.; Ferrari, A. L. 2004. Evolução Geológica do Rift Continental do Sudeste do Brasil. In: Manteso-Neto, V.; Bartorelli, A.; Carneiro, C. D. R.; Neves, B. B. B. Geologia do Continente SulAmericano: Evolução da Obra de Fernando Marques de Almeida. Beca, São Paulo, 384-405.

Telford, W.M.; Geldart, L.P.; Sheriff, R.E., 1990. Applied Geophysics, Cambridge University Press, $2^{\text {nd }}$ edition. 
9.2 Artigo publicado pela revista International Journal of Geosciences 


\section{Electrical Resistivity Tomography and TDEM Applied to Hydrogeological Study in Taubaté Basin, Brazil}

\section{Rodrigo Corrêa Rangel1, Jorge Luís Porsani' ${ }^{1}$, Cassiano Antonio Bortolozo ${ }^{1,2}$, Luiz Rodrigo Hamada ${ }^{1}$}

${ }^{1}$ Universidade de São Paulo, Instituto de Astronomia, Geofísica e Ciências Atmosféricas, Departamento de Geofísica, São Paulo, Brazil

${ }^{2}$ CEMADEN-National Center for Natural Disaster Monitoring and Alert, General Coordination of Research and Development, São José dos Campos, Brazil

Email: rodrigo.rangel@iag.usp.br, jorge.porsani@iag.usp.br, cassianoab@gmail.com, luizhamada@iag.usp.br

How to cite this paper: Rangel, R.C., Porsani, J.L., Bortolozo, C.A. and Hamada, L.R. (2018) Electrical Resistivity Tomography and TDEM Applied to Hydrogeological Study in Taubaté Basin, Brazil. International Journal of Geosciences, 9, 119-130. https://doi.org/10.4236/ijg.2018.92008

Received: January 5, 2018

Accepted: February 23, 2018

Published: February 26, 2018

Copyright $\odot 2018$ by authors and Scientific Research Publishing Inc. This work is licensed under the Creative Commons Attribution International License (CC BY 4.0).

http://creativecommons.org/licenses/by/4.0/

\begin{abstract}
This research applies Electrical Resistivity Tomography (ERT) and Time Domain Electromagnetic Method (TDEM) to study the hydrogeology of the Taubaté basin, which is characterized by half-grabens with about $850 \mathrm{~m}$ of maximum sediments thickness. The study area is in Taubaté city, São Paulo State, Brazil, where the Taubaté aquifer is an important water source. The Taubaté Group is the main sedimentary package of the basin; it is formed mainly by shales that form aquicludes, and thin layers of sandstones that form the aquifer. There are 40 groundwater exploration wells in Taubate city that provide important information. The study purpose is to characterize the geoelectrical stratigraphy of the subsurface to locate the contact between the Quaternary and Tertiary sediments and to identify the Taubaté aquifer. The ERT is used for shallow investigations (tens of meters) and the TDEM can reach a great investigation depth (hundreds of meters). Therefore, these geophysical methods are complementary. The ERT data were acquired with the pole-dipole array with $20 \mathrm{~m}$ of electrodes spacing and $400 \mathrm{~m}$ length, and the TDEM data with the central-loop array with a $200 \times 200 \mathrm{~m}$ transmitter loop. The results permit to define the contact between the Quaternary and Tertiary sediments around $15 \mathrm{~m}$ depth, the Pindamonhangaba Formation between 15 $\mathrm{m}$ and $30 \mathrm{~m}$ depth and the Taubate Group between $30 \mathrm{~m}$ and $300 \mathrm{~m}$ depth. The TDEM method defined the Taubate Group as a single geoelectric layer because the shale and the sandstone layers are all very conductive. The basement is formed by gneiss, which is a very resistive rock. The TDEM method is not able to identify a high conductor/resistor contrast. Overall, the results are
\end{abstract}


consistent with the known geology and the wells information.

\section{Keywords}

Electrical Resistivity Tomography (ERT), Time Domain Electromagnetic (TDEM), Hydrogeophysics, Taubaté Basin, Brazil

\section{Introduction}

In this research, the electrical resistivity tomography (ERT) and time domain electromagnetic (TDEM) methods were applied to a hydrogeological study in Taubaté basin, São Paulo State, Brazil (Figure 1), where the Taubaté aquifer is an important water supply for the region.

Each method has advantages and limitations in terms of investigation depth and resolution. The ERT method is normally used in shallow investigations (tens of meters) due to logistical limitation because it requires a long aperture to achieve greater depths. On the other hand, the TDEM method has a low resolution for the shallow layers; it is used in deep investigations (hundreds of meters) and can define conductive layers [1] [2].

Both methods are widely applied in hydrogeophysical studies, because of rapid data acquisition, relatively low cost, and reliability. They have the potential to identify aquifers, aquicludes, and aquitards, which normally, in Brazil, are more conductive than the surrounding layers. They are also sensitive to geological properties, like clay content.

Electrical resistivity method [3] [4] [5] [6] and TDEM [7]-[18] have been successfully applied individually and also jointly [19] [20] [21] [22] [23] for hydrogeophysical investigations in many places around the world, including Brazil [11] [13] [14] [16] [17] [19] [21] [22]. Their applications also include mining, geotechnical and environmental studies [24] [25], among others.

The study aims to characterize the geoelectrical stratigraphy of the subsurface to locate the contact of the Quaternary and Tertiary sediments and identify the Taubaté aquifer. Furthermore, it aims to contribute to the geoelectrical methods interpretation in a hydrogeological context like Taubaté basin and to collaborate with the hydrogeophysical studies in São Paulo State.

\section{Study Area}

The studied area is in Taubaté city, São Paulo State, Brazil, which is located on the central portion of the Taubaté Basin (Figure 1(a)). Taubaté is a medium-sized city with about 308,000 inhabitants, where the demand for water has been increasing.

The data acquisitions were conducted on a cattle farm around $6 \mathrm{~km}$ from the city center. The terrain was practically flat, which has the advantage of not requiting topography correction on data processing. 


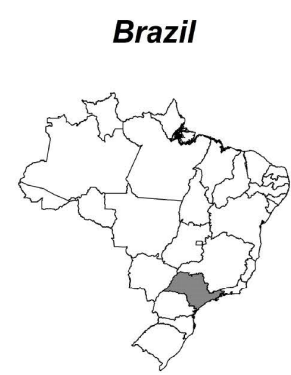

- Wells

* Taubaté City

จ São Paulo City

- Study Area

$\square \quad$ Taubaté Basin
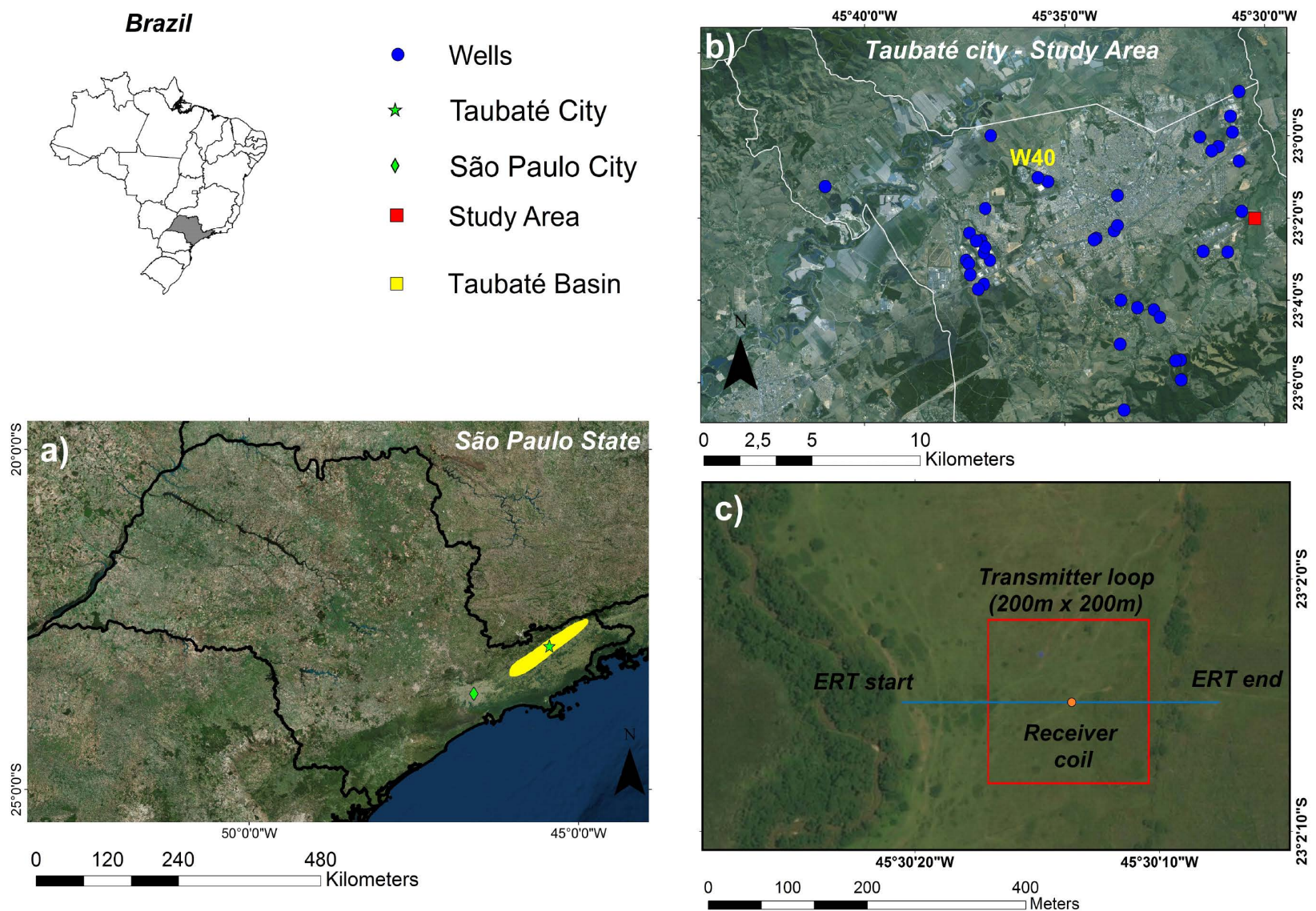

Figure 1. Study area location. (a) Taubaté Basin and Taubaté city, São Paulo state, Brazil; (b) Study area and wells (40); (c) ERT line and TDEM loop.

\subsection{Geological Setting}

According to [26], Taubaté basin is the largest basin of the Continental Rift of Southeast Brazil (CRSB) with $170 \mathrm{~km}$ length and $20 \mathrm{~km}$ width. The basin is elongated in NE-SW direction and presents normal faults in NW-SE direction. The basin is related to Tertiary extensional tectonics and it is characterized by a series of half-grabens with $850 \mathrm{~m}$ of maximum sediments thickness [27].

Figure 2 presents a geological map and the stratigraphic chart of the basin. It was developed over Precambrian gneisses and granites [28]. The sedimentary fill is basically continental and can be divided in two phases: the first, syntectonic to the rift, with the deposition of the Taubaté Group; and the second, posterior to diastrophic tectonics, with the deposition of the Pindamonhangaba Formation and alluvial and colluvial deposits [28].

The main sedimentary package is the Taubate Group, which is subdivided by the Resende, Tremembé and São Paulo formations. Taubaté Group is formed mainly by shales and sandstones. Resende Formation is the most abundant package of the Taubaté Group [27]. Pindamonhangaba Formation was deposited in the Neogene in a fluvial meandering environment. Finally, in the Quaternary, there are alluvial, colluvial, colluvial-alluvial and talus deposits. 


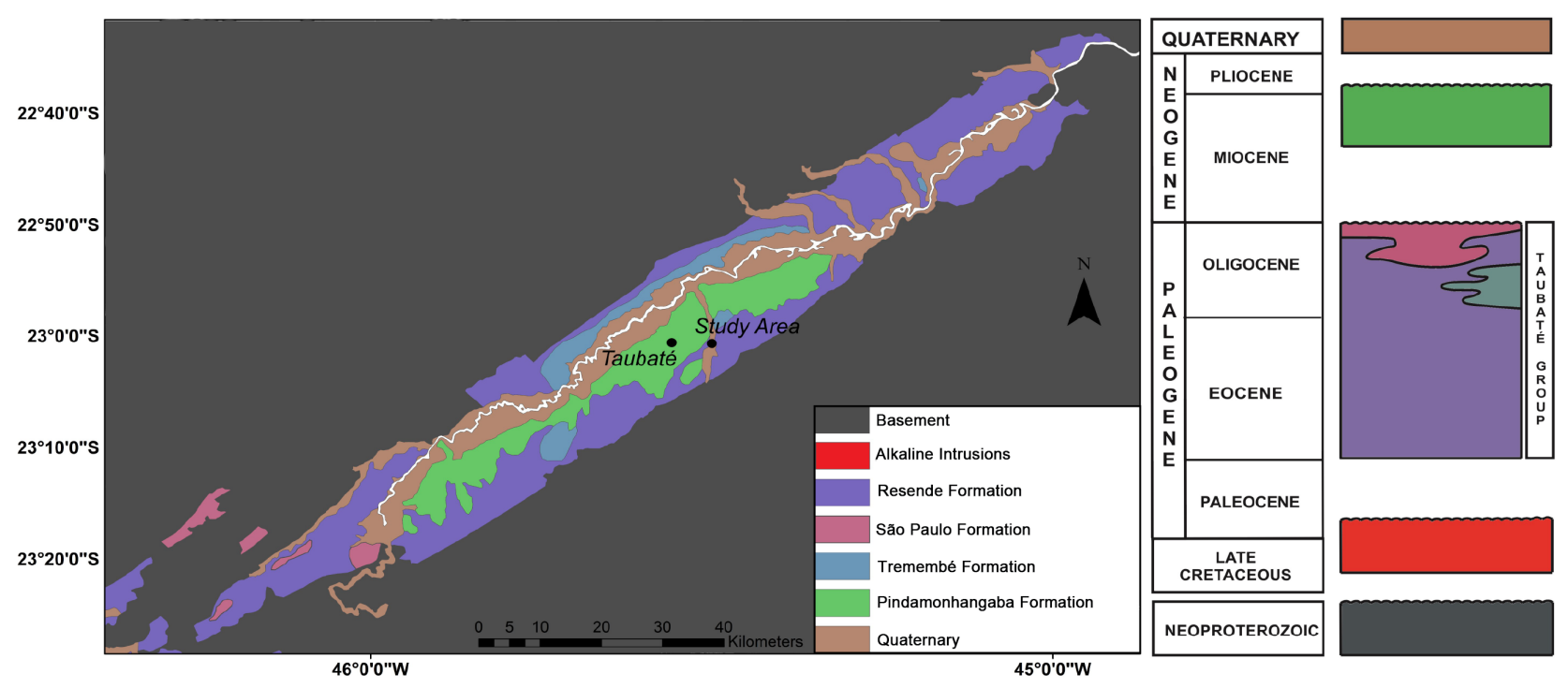

Figure 2. Geological map and stratigraphic chart of Taubaté basin (adapted from [27]).

\subsection{Taubaté Aquifer}

Taubate Aquifer occurs mainly in two areas of the basin, in the southwest and in the northeast. Between these two regions, where Taubate city is located, there is a compartment filled predominantly by argillites and shales with low permeability, which presents aquiclude characteristics [29]. As a result of its depositional environments, the aquifer is a multilayer type, with alternation of sandy or aquifer layers, associated with fluvial facies, and clayey or confining layers, associated with lacustrine or floodplain facies [29]. In Taubaté city, the saturated thickness of the aquifer varies from $200 \mathrm{~m}$ to $300 \mathrm{~m}$. According to [30], the central region of the basin, where Taubaté is located, presents unfavorable characteristics for groundwater exploration, with flow rates lower than $10 \mathrm{~m}^{3} / \mathrm{h}$.

\subsection{Wells}

There are 40 wells in Taubaté city available in the Groundwater Information System (SIAGAS) of the Geological Service of Brazil (CPRM) [31] database, which include the coordinates, depth, water use and a geological profile for each well. Figure 1(b) shows the location of the wells. All wells are tubular and the water is mainly used for domestic or industrial supply. Some of them present the water level and flow rate, which in general are lower than $10 \mathrm{~m}^{3} / \mathrm{h}$, being consistent with [30]. Six wells reached the basement depth, which is formed by gneisses.

The closest well is around $750 \mathrm{~m}$ distant and the deepest (W40) (that reaches $650 \mathrm{~m}$ depth) is around $9.5 \mathrm{~km}$ distant from the study area. Table 1 shows W40 lithological description. Down to $11 \mathrm{~m}$ depth represents the Quaternary sediments. The sandstone between $11 \mathrm{~m}$ and $16 \mathrm{~m}$ depth probably represents the Pindamonhangaba formation. The rocks between 16 and $510 \mathrm{~m}$ depth represent the Taubaté Group, which is formed mainly by shales, and bellow $510 \mathrm{~m}$ depth is the basement formed by gneisses. 
Table 1. Lithological description of the deepest well (W40) in Taubaté city [31].

\begin{tabular}{ccc}
\hline From $(\mathrm{m})$ & To $(\mathrm{m})$ & Soil/Lithology \\
\hline 0 & 4 & Soil \\
4 & 11 & Clay \\
11 & 16 & Sandstone \\
16 & 485 & Shales \\
485 & 493 & Clayey sandstone \\
493 & 510 & Shales \\
510 & 650 & Gneiss \\
\hline
\end{tabular}

In general, from all wells lithological descriptions, the Taubaté Group is formed mainly by shales intercalated with thin layers of sandstones. Therefore, in the Taubaté Group, the shales form aquicludes and the sandstones form the aquifer.

\section{Geophysical Methods}

In this research were applied two geophysical methods: Electrical Resistivity Tomography (ERT) and Time Domain Electromagnetic (TDEM). Both methods investigate the same physical property of subsurface materials, the electrical resistivity $(\rho)$.

In the ERT method, the investigation is done through the injection of electric currents and, in the TDEM method, through the electromagnetic induction in conductive materials in the subsurface. Both methods use artificial sources and the response is measured at the surface. Thus, it is possible to relate the electrical resistivity distribution with the geology or variations in the lithological composition, for example, the presence of water, fractures, and mineralogy.

\subsection{Electrical Resistivity Tomography (ERT)}

The ERT method consists of injecting an electric current $(I)$ into the ground through metallic electrodes and measuring the resulting potential $(\Delta V)$ by other electrode pairs. In this way, it is possible to obtain the electrical resistivity distribution of the subsurface.

Electrical resistivity values can be estimated because the spatial arrangement of the electrodes is known. According to [32], the apparent electrical resistivity $\left(\rho_{a}\right)$ is given by:

$$
\rho_{a}=K \frac{\Delta V}{I}
$$

where $K$ is the geometric factor, which depends on the electrodes array. The investigation depth depends on the subsurface resistivity and the electrodes separation, i.e., greater depths are achieved by increasing the array size.

The ERT is used to map the lateral variation of the resistivity as a function of depth (2D). There are several types of electrode arrays, however, in this research 
was only used the Pole-Dipole array with six investigation levels.

The ERT data was acquired in June 2016 with the Syscal Pro (Iris Instruments) equipment. The imaging line was $400 \mathrm{~m}$ length (Figure $1(\mathrm{c})$ ) and with 20 $\mathrm{m}$ of electrodes spacing. The data were inverted with the RES2DINV software [33], which uses the field data to automatically determine a two-dimensional model of the subsurface resistivity.

\subsection{Time Domain Electromagnetic (TDEM)}

The TDEM method is based on the electromagnetic induction principle and it is used to estimate the resistivity variation as a function of depth [1] [2]. The investigation is performed by measuring the decay of an induced secondary magnetic field in the subsurface due to the variation of a primary magnetic field generated on the surface.

According to [2], the relationship between the secondary magnetic field variation $\left(\partial B_{z} / \partial t\right)$ and the apparent resistivity $\left(\rho_{a}\right)$ is given by:

$$
\rho_{a}=\frac{1}{\pi}\left(\frac{I \pi a^{2}}{20 \partial B_{z} / \partial t}\right)^{2 / 3}\left(\frac{\mu_{0}}{t}\right)^{5 / 3}
$$

where $I(\mathrm{~A})$ is current, $a(\mathrm{~m})$ the loop radius, $\mu_{0}$ the vacuum magnetic permeability and $t(\mathrm{~s})$ the time.

There are several acquisition arrays and field procedures. The array configuration, size, and other parameters depend on the purpose of the research. In this research, the central loop array (Figure 1(c)) was used, where the receiver coil is placed in the transmitter loop center. It is one of the most popular TDEM arrays, having the advantage of a good signal-to-noise ratio.

The TDEM method is sensitive to conductive layers, because of the current flows that are induced in these layers. The investigation depth depends on the subsurface resistivity, the more resistive the medium, the faster the secondary field diffuses and vice versa [2]. The investigation depth also depends on the magnetic dipole moment ( $M=I A$ ) of the transmitter loop. Therefore, increasing $M$, it is possible to reach greater investigation depths. In this research, the acquisition was done with $I=17.5 A$ and $200 \times 200 \mathrm{~m}$ square transmitter loop (Figure 1(c)).

This method has an insignificant influence from natural sources noise. The main noise sources are man-made artifacts such as power transmission lines, cables and buried pipes and metal fences near the data acquisition area [2]. The transmitter induces electric currents in these conductors which, as consequence, interfere in the induced secondary currents in subsurface materials. No noise source influenced the acquisition, because the electric power lines and metal fences were more than $100 \mathrm{~m}$ away, which is considered a safe distance [2].

The data were acquired in April 2016 with the PROTEM 57-MK2 D [34] equipment, which consists of a transmitter that is connected to a power generator to produce the primary electromagnetic field; and a $3 \mathrm{D}$ receiver coil of about $1 \mathrm{~m}$ diameter and $200 \mathrm{~m}^{2}$ of effective area that is connected to a computer to 
record the signal of the secondary electromagnetic field induced in the subsurface. The main acquisition parameters were: repetition rates of $30 \mathrm{~Hz}, 7.5 \mathrm{~Hz}$ and $3 \mathrm{~Hz}$, integration time of $30 \mathrm{~s}$ and average of three points measured for each repetition rate.

The data were inverted with the Curupira software [35]. The inversion process consists of determining the electrical resistivity and thickness of the subsurface layers from the measured data.

\section{Results Analysis}

Figure 3 shows the ERT inversion result, where: a) the measured apparent resistivity pseudosection, b) the calculated apparent resistivity pseudosection and c) the inverted geoelectric model. The RMS error is $1.59 \%$ after 6 iterations. The black line in the inverted model (Figure 3(c)) represents the contact between the Quaternary and Tertiary sediments. The Quaternary sediments are formed by soil, alluvial and colluvial deposits going down to maximum around $30 \mathrm{~m}$ depth in the left side; it is a very resistive zone, with the resistivity varying from more than $200 \Omega \cdot \mathrm{m}$ to $80 \Omega \cdot \mathrm{m}$. Below that, the Pindamonhangaba Formation is formed by sandstones and presents resistivity between the $80 \Omega \cdot \mathrm{m}$ and $30 \Omega \cdot \mathrm{m}$. The white line represents the interface between the Pindamonhangaba formation and the Taubaté Group, which is very conductive, between $30 \Omega \cdot \mathrm{m}$ and 10 $\Omega \cdot \mathrm{m}$.

Figure 4 shows the TDEM inversion result. Figure 4(a) presents the apparent resistivity $(\Omega \cdot \mathrm{m})$ versus time $(\mathrm{ms})$ showing the measured data and the adjusted curve; and Figure 4(b), the inverted geoelectric model, depth (m) versus resistivity $(\Omega \cdot \mathrm{m})$. The adjustment error is $1.7 \%$ after 1000 iterations.

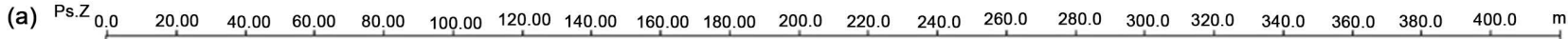

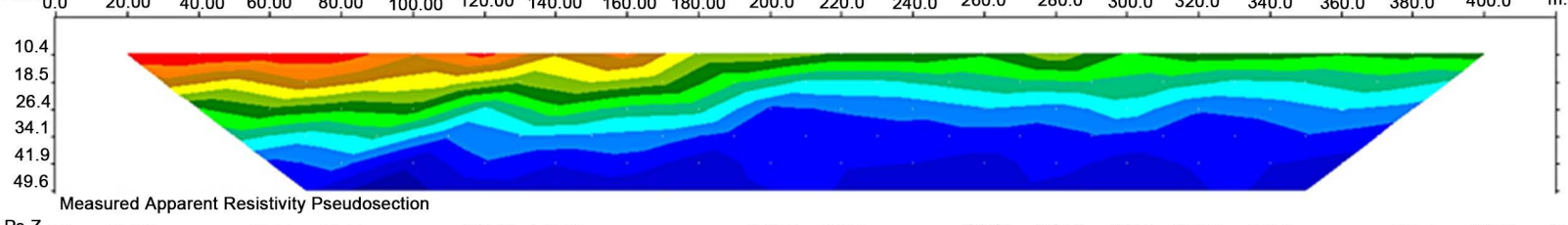

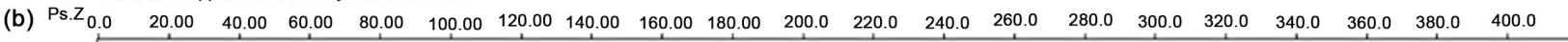

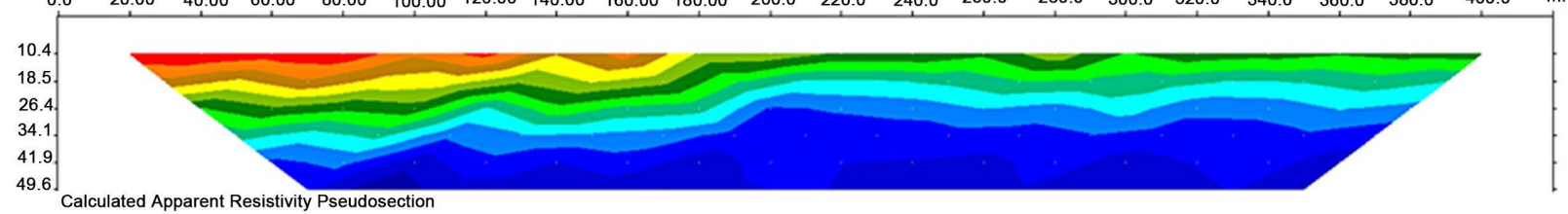

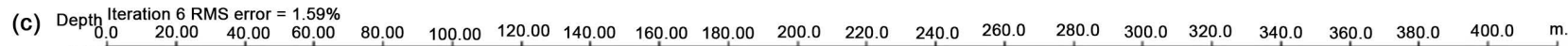

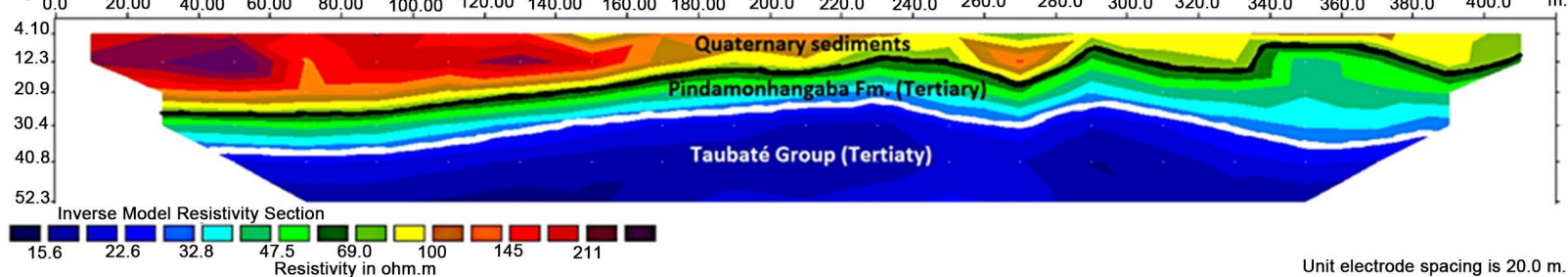

Figure 3. ERT inversion result. (a) Measured apparent resistivity pseudosection; (b) Calculated apparent resistivity pseudosection; c) Inverted geoelectric model. 
(a)

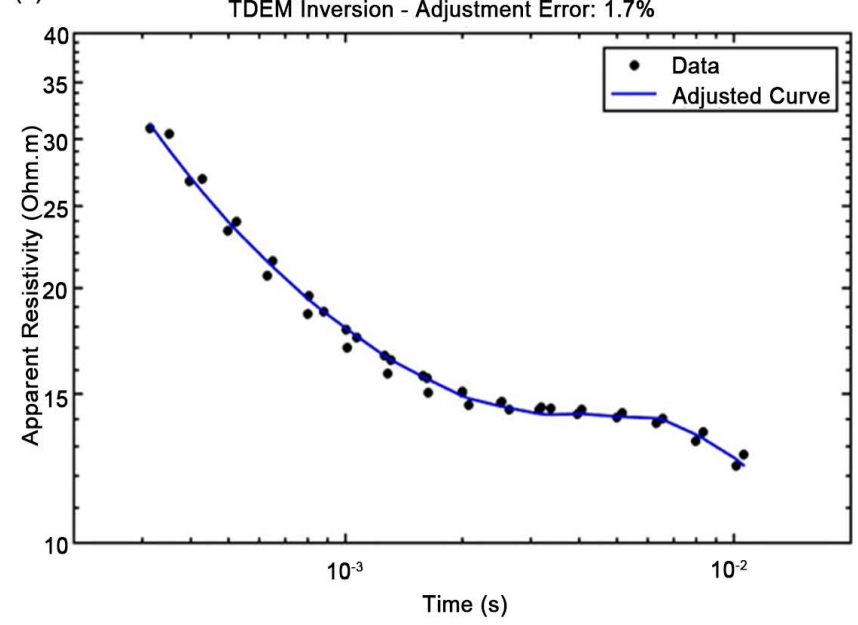

(b)

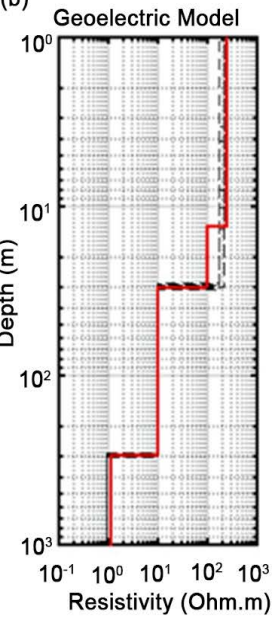

Figure 4. TDEM inversion result. (a) Feild data points with the adjusted curve on Curupira software; (b) The inverted geoelectric model.

In the geoelectric model, there are 3 geoelectrical layers down to $300 \mathrm{~m}$ depth. The first layer down to $13 \mathrm{~m}$ is resistive, with more than $200 \Omega \cdot \mathrm{m}$, representing the Quaternary sediments. The second layer, from $13 \mathrm{~m}$ to $30 \mathrm{~m}$ depth, has a resistivity around $100 \Omega \cdot \mathrm{m}$, which represent the Pindamonhangaba formation. The third layer, from $30 \mathrm{~m}$ to around $300 \mathrm{~m}$ is very conductive, around $10 \Omega \cdot \mathrm{m}$, representing the Taubaté Group. The TDEM result is consistent with the ERT result.

The Taubate Group is very conductive, therefore the signal concentrates and dissipates in this layer, not being able to induce an electromagnetic field in the layer below, which is the resistive basement. The basement is formed by gneisses, which is a very resistive rock, with resistivity values in the order of thousands of $\Omega \cdot \mathrm{m}[36]$.

Table 2 summarizes the ERT and TDEM results interpretation. The first layer is interpreted as the Quaternary sediments, the second layer is the Pindamonhangaba formation and the third layer represents the Taubate Group. The resistivity values were estimated based on the ERT (Figure 3(c)) and the TDEM (Figure 4(b)) inverted geoelectric models. These results are coherent and they agree with wells information.

\section{Conclusions}

The results have shown a great potential for the application of the combined ERT and TDEM methods to the geoelectrical characterization of the Taubaté Basin, making it possible to identify the contact between the Quaternary and Tertiary sediments and to define the Taubaté Group, which stores the Taubaté aquifer.

Both data inversions obtained a low RMS error $(<2 \%)$, resulting in a geoelectrical model coherent with the known geology. 
Table 2. Interpretation of the ERT and TDEM inverted geoelectric models.

\begin{tabular}{cccc}
\hline Depth $(\mathrm{m})$ & Resistivity $(\Omega \cdot \mathrm{m})$ & Interpretation & Period/Epoch \\
\hline $0-15$ & $250-100$ & Soil, alluvial and colluvial deposits & Quaternary \\
$15-30$ & $100-30$ & Pindamonhangaba Formation & Neogene \\
$30-300$ & $30-10$ & Shales and sandstones (Taubaté Group) & Eocene-Oligocene \\
\hline
\end{tabular}

The ERT was able to image the shallow sedimentary layers until $50 \mathrm{~m}$ depth and the TDEM provided information until about $300 \mathrm{~m}$ depth, allowing defining the conductive layer represented by the Taubaté Group.

The Taubaté Group is approximately $270 \mathrm{~m}$ thick, mainly formed by shale and thin sandstone layers. The shales form the aquiclude and the sandstones form the aquifer. Both are very conductive, so the TDEM method identified them as a single conductive layer.

The Taubaté Group is a very conductive layer and, below it, the basement is very resistive. Therefore, in this research, the TDEM method was not able to pass through the sedimentary conductive layer; however, based on the well information, the basement top is probably around $300 \mathrm{~m}$ depth, but more studies are needed.

Both geophysical methods have the advantage of rapid data acquisition, relatively low cost, and they are reliable to identify aquifers and aquicludes. Other geophysical studies have already been done in Taubaté basin. For example, Cogné et al. [37] have reinterpreted 11 seismic profiles of the basin to study its tectonic setting; and Padilha et al. [38] have applied the magnetotelluric method across the basin to obtain a deep $(20 \mathrm{~km}) 2 \mathrm{D}$ geoelectrical model, which identified the conductive sediments of Taubaté Group with low resolution. However, the present research is the first in terms of a hydrogeophysical study in Taubate basin.

\section{Acknowledgements}

RCR and LRH thank CAPES (Coordenação de Aperfeiçoamento de Pessoal de Nível Superior) for providing the research scholarships. JLP thanks to FAPESP (Fundação de Amparo à Pesquisa do Estado de São Paulo) (grants: 2009/08466-3 and 2012/15338-4) and CNPq (Conselho Nacional de Desenvolvimento Científico e Tecnológico) (grants: 301692/2013-0 and 406653/2013-5), both for providing financial support to develop this research. $\mathrm{CAB}$ thanks to CNPq for the Postdoctoral scholarship (150230/2016-8). We thank IAG/USP for providing the infrastructure support. We thank Ernande Costa Santos, Marcelo Cesar Stangari and the students for helping in geophysical data acquisition.

\section{References}

[1] McNeill, J.D. (1994) Principles and Application of Time Domain Electromagnetic Techniques for Resistivity Sounding. Technical Note TN-27, Geonics Ltd., Ontario.

[2] Christiansen, A.V., Auken, E. and Sørensen, K. (2006) The Transient Electromag- 
netic Method. In: Kirsch, R., Ed., Groundwater Geophysics-A Tool for Hydrogeology, GSW Ltd., Aarhus, 179-225. https://doi.org/10.1007/3-540-29387-6_6

[3] Froese, D.G., Smith, D.G. and Clement, D.T. (2005) Characterizing Large River History with Shallow Geophysics: Middle Yukon River, Yukon Territory and Alaska. Geomorphology, 67, 391-406.

https://doi.org/10.1016/j.geomorph.2004.11.011

[4] Chandra, S., Rao, V.A., Krishnamurthy, N.S., Dutta, S. and Ahmed, S. (2006) Integrated Studies for Characterization of Lineaments Used to Locate Groundwater Potential Zones in a Hard Rock Region of Karnataka, India. Hydrogeology Journal, 14, 767-776. https://doi.org/10.1007/s10040-005-0480-3

[5] Fowler, D.E. and Moysey, S.M.J. (2011) Estimation of Aquifer Transport Parameters from Resistivity Monitoring Data within a Coupled Inversion Framework. Journal of Hydrology, 409, 545-554. https://doi.org/10.1016/j.jhydrol.2011.08.063

[6] Yeh, H.F., Lin, H.I., Wu, C.S., Hsu, K.C., Lee, J.W. and Lee, C.H. (2015) Electrical Resistivity Tomography Applied to Groundwater Aquifer at Downstream of Chih-Ben Creek Basin, Taiwan. Environmental Earth Sciences, 73, 4681-4687. https://doi.org/10.1007/s12665-014-3752-1

[7] Fitterman, D.V. and Stewart, M.T. (1986) Transient Electromagnetic Sounding for Groundwater. Geophysics, 51, 955-1005. https://doi.org/10.1190/1.1442158

[8] Goldman, M., Du Plooy, A. and Eckard, M. (1994) On Reducing Ambiguity in the Interpretation of Transient Electromagnetic Sounding Data. Geophysical Prospecting, 42, 3-25. https://doi.org/10.1111/j.1365-2478.1994.tb00192.x

[9] Danielsen, J.E., Auken, E., Jorgensen, F., Sondergaard, V. and Sørensen, K.I. (2003) The Application of the Transient Electromagnetic Method in Hydrogeophysical Surveys. Journal of Applied Geophysics, 53, 181-198. https://doi.org/10.1016/j.jappgeo.2003.08.004

[10] Jørgensen, F., Sandersen, P.B.E. and Auken, E. (2003) Imaging Buried Quaternary Valleys Using Transient Electromagnetic Method. Journal of Applied Geophysics, 53, 199-213. https://doi.org/10.1016/j.jappgeo.2003.08.016

[11] Carrasquilla, A.A.G. and Ulugergerli, E. (2006) Evaluation of the Transient Electromagnetic Geophysical Method for Stratigraphic Mapping and Hydrogeological Delineation in Campos Basin, Brazil. Revista Brasileira de Geofísica, 24, 333-341. https://doi.org/10.1590/S0102-261X2006000300003

[12] Nielsen, L., Jørgensen, N.O. and Gelting, P. (2007) Mapping of the Freshwater Lens in a Coastal Aquifer on the Keta Barrier (Ghana) by Transient Electromagnetic Soundings. Journal of Applied Geophysics, 62, 1-15. https://doi.org/10.1016/j.jappgeo.2006.07.002

[13] Porsani, J.L., Bortolozo, C.A., Almeida, E.R., Sobrinho, E.N.S. and Santos, T.G. (2012) TDEM Survey in Urban Environmental for Hydrogeological Study at USP Campus in São Paulo City, Brazil. Journal of Applied Geophysics, 76, 102-108. https://doi.org/10.1016/j.jappgeo.2011.10.001

[14] Porsani, J.L., Almeida, E.R. and Bortolozo, C.A. (2012) TDEM Survey in an Area of Seismicity Induced by Water Wells in Paraná Sedimentary Basin, Northern São Paulo State, Brazil. Journal of Applied Geophysics, 82, 75-83. https://doi.org/10.1016/j.jappgeo.2012.02.005

[15] Martínez-Moreno, F.J., Santos, F.A.M., Madeira, J., Bernardo, I., Soares, A., Esteves, M. and Adão, F. (2016) Water Prospection in Volcanic Islands by Time Domain Electromagnetic (TDEM) Surveying: The Case Study of the Islands of Fogo and Santo Antão in Cape Verde. Journal of Applied Geophysics, 134, 226-234. 
https://doi.org/10.1016/j.jappgeo.2016.09.020

[16] Almeida, E.R., Porsani, J.L., dos Santos, F.A.M. and Bortolozo, C.A. (2017) 2D TEM Modeling for a Hydrogeological Study in the Paraná Sedimentary Basin, Brazil. International Journal of Geosciences, 8, 693-710. https://doi.org/10.4236/ijg.2017.85038

[17] Campaña, J.D.R., Porsani, J.L., Bortolozo, C.A., Oliveira, G.S. and Santos, F.A.M. (2017) Inversion of TEM Data and Analysis of the 2D Induced Magnetic Field Applied to the Aquifers Characterization in the Paraná Basin, Brazil. Journal of Applied Geophysics, 138, 233-244. https://doi.org/10.1016/j.jappgeo.2017.01.024

[18] Costabel, S., Siemon, B., Houben, G. and Günther, T. (2017) Geophysical Investigation of a Freshwater Lens on the Island of Langeoog, Germany-Insights from Combined HEM, TEM and MRS Data. Journal of Applied Geophysics, 136, 231-245. https://doi.org/10.1016/j.jappgeo.2016.11.007

[19] Meju, M.A., Fontes, S.L., Oliveira, M.F.B., Lima, J.P.R., Ulugergerli, E.U. and Carrasquilla, A.A.G. (1999) Regional Aquifer Mapping using Combined VES-TEMAMT-EMAP Methods in the Semi-Arid Eastern Margin of Parnaiba Basin, Brazil. Geophysics, 64, 337-356. https://doi.org/10.1190/1.1444539

[20] Albouy, Y., Andrieux, P., Rakotondrasoa, G., Ritz, M., Descloitres, M., Join, J.L. and Rasolomanana, E. (2001) Mapping Coastal Aquifers by Joint Inversion of DC and TEM Soundings-Three Case Histories. Ground Water, 39, 87-97. https://doi.org/10.1111/j.1745-6584.2001.tb00354.x

[21] Bortolozo, C.A., Porsani, J.L., Santos, F.A.M. and Almeida, E.R. (2015) VES/TEM 1D Joint Inversion by Using Controlled Random Search (CRS) Algorithm. Journal of Applied Geophysics, 112, 157-174. https://doi.org/10.1016/j.jappgeo.2014.11.014

[22] Bortolozo, C.A., Couto, M.A., Porsani, J.L., Almeida, E.R. and Santos, F.A.M. (2014) Geoelectrical Characterization Using Joint Inversion of VES/TEM Data: A Case Study in Paraná Sedimentary Basin, São Paulo State, Brazil. Journal of Applied Geophysics, 111, 33-46. https://doi.org/10.1016/j.jappgeo.2014.09.009

[23] Martínez-Moreno, F.J., Santos, F.A.M., Bernardo, I., Farzamian, M., Nascimento, C., Fernandes, J., Casal, B. and Riberio, J.A. (2017) Identifying Seawater Intrusion in Coastal Areas by Means of $1 \mathrm{D}$ and quasi-2D Joint Inversion of TDEM and VES Data. Journal of Hydrology, 552, 609-619. https://doi.org/10.1016/j.jhydrol.2017.07.026

[24] Schmutz, M., Albouy, Y., Guerin, R., Maquaire, O., Vassal, J., Schott, J.J. and Descloitres, M. (2000) Joint Electrical and Time Domain Electromagnetism (TDEM) Data Inversion Applied to the Super Sauze Earthflow (France). Surveys in Geophysics, 21, 371-390. https://doi.org/10.1023/A:1006741024983

[25] Schmutz, M., Guérin, R., Andrieux, P. and Maquaire, O. (2009) Determination of the 3D Structure of an Earthflow by Geophysical Methods: The Case of Super Sauze, in the French Southern Alps. Journal of Applied Geophysics, 68, 500-507. https://doi.org/10.1016/j.jappgeo.2008.12.004

[26] Riccomini, C., Sant'Anna, L.G. and Ferrari, A.L. (2004) Evolução geológica do Rift Continental do Sudeste do Brasil. In: Mantesso Neto, V., Bartorelli, A., Carneiro, C.D.R. and Brito-Neves, B.B., Eds., Geologia do Continente Sul-Americano: Evolução da Obra de Fernando Flávio Marques de Almeida, Ed. Beca, São Paulo, 383-405.

[27] Marques, A. (1990) Evolução tectono-sedimentar e perspectivas exploratórias da Bacia de Taubaté, São Paulo, Brasil. Boletim de Geociências da Petrobrás, 4, 253-262. 
[28] Riccomini, C. (1989) O Rift Continental do Sudeste do Brasil. PhD Thesis, Institute of Geosciences, University of São Paulo, São Paulo.

[29] DAEE (Departamento de Águas e Energia Elétrica) (1977) Estudo de águas subterrâneas da região administrativa 3 (São José dos Campos e Faixa Litorânea). Governo do Estado de São Paulo. São Paulo.

[30] DAEE (Departamento de Águas e Energia Elétrica), IG (Instituto Geológico), IPT (Instituto de Pesquisas Tecnológicas) and CPRM (Serviço Geológico do Brasil) (2005) Mapa de águas subterrâneas do Estado de São Paulo. São Paulo.

[31] Groundwater Information System (SIAGAS) of the Geological Service of Brazil (CPRM). http://siagasweb.cprm.gov.br/layout/

[32] Koefoed, O. (1979) Geosounding Principles 1: Resistivity Sounding Measurements. Elsevier Science Publishing Company, Amsterdam.

[33] Loke, M.H. (2004) Res2Dinv v. 3.54 for Windows 98/Me/2000/NT/XP. Rapid 2D Resistivity and IP Inversion Using the Least-Squares Method. Software Manual.

[34] Geonics (2009) PROTEM 57-MK2 D Operating Manual. Geonics Limited, Mississauga, Ontario.

[35] Bortolozo, C.A. and Porsani, J.L. (2012) "CURUPIRA V1.0". Software de inversão conjunta 1D de sondagens SEV/TDEM. Registro de Programa de Computador número 12988-1. Revista da Propriedade Industrial, 2165, 145.

[36] Telford, W.M., Geldart, L.P. and Sheriff, R.E. (1990) Applied Geophysics. 2nd Edition, Cambridge University Press, Cambridge. https://doi.org/10.1017/CBO9781139167932

[37] Cogné, N., Coboold, P.R., Riccomini, C. and Gallagher, K. (2013) Tectonic Setting of the Taubaté Basin (Southeastern Brazil): Insights from Regional Seismic Profiles and Outcrop Data. Journal of South American Earth Sciences, 42, 194-204. https://doi.org/10.1016/j.jsames.2012.09.011

[38] Padilha, A.L., Vitorello, I. and Brito, P.M.A. (2002) Magnetotelluric Soundings across the Taubaté Basin, Southeast Brazil. Earth, Planets and Space, 54, 617-627. https://doi.org/10.1186/BF03353050 
9.3 Tutorial de Inversão Conjunta 2D CE/CTDEM 
Universidade de São Paulo

Instituto de Astronomia, Geofísica e Ciências Atmosféricas

Departamento de Geofísica

\section{Inversão Conjunta 2D CE/CTDEM}

\section{Tutorial}

Rodrigo Corrêa Rangel

Cassiano Antonio Bortolozo

Jorge Luis Porsani

São Paulo 


\section{Sumário}

1. Introdução a metodologia de Inversão Conjunta 2D CE/CTDEM ..........................................95

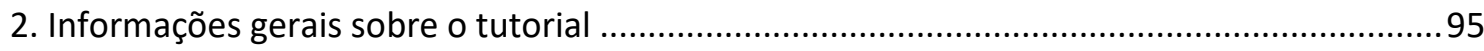

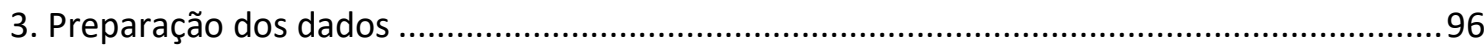

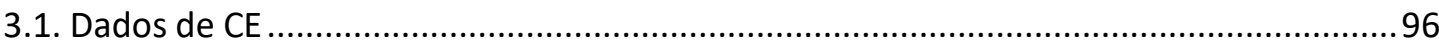

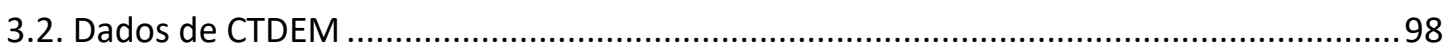

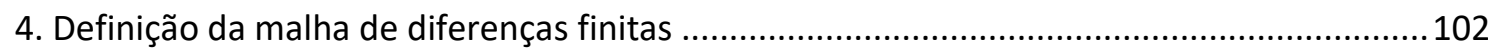

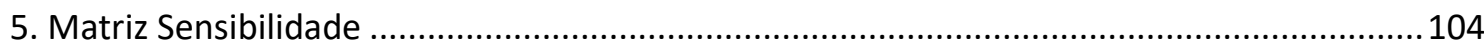

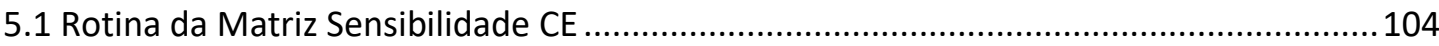

5.2. Rotina da Matriz Sensibilidade CTDEM ………........................................................

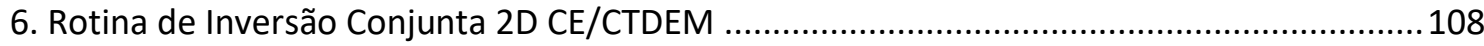

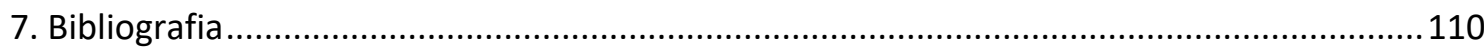




\section{Introdução a metodologia de Inversão Conjunta 2D CE/CTDEM}

A metodologia de Inversão Conjunta 2D de dados de caminhamento elétrico (CE) e caminhamento TDEM (CTDEM) foi desenvolvida de forma pioneira por Bortolozo (2016).

A modelagem do CE foi realizada com base no artigo de Dey e Morrison (1979) e a modelagem do CTDEM com base no artigo de Oristaglio e Hohmann (1984). Ambas as formulações utilizaram a modelagem 2D por diferenças finitas. O modelo geoelétrico é representado por uma seção discretizada em polígonos, onde cada polígono possui um valor de resistividade elétrica independente dos demais. Desse modo o problema consiste em determinar a distribuição dos campos elétricos e eletromagnéticos na seção discretizada devido a uma fonte conhecida. 0 algoritmo de inversão conjunta 2D foi desenvolvido a partir do método de Occam para modelos suavizados.

Um conjunto de rotinas foram criadas no Matlab para realizar a Inversão Conjunta 2D CE/CTDEM e neste tutorial explicamos o passo a passo de como implementá-las. Para maiores detalhes sobre a metodologia, consultar a bibliografia.

\section{Informações gerais sobre o tutorial}

Segue uma lista de informações iniciais importantes sobre o tutorial:

a) Primeiro deve-se rodar as rotinas para obter as Matrizes Sensibilidade CE e CTDEM e depois a Inversão Conjunta CE/CTDEM.

b) A Matriz Sensibilidade CE leva horas para rodar, no caso de Taubaté foram 35 horas.

c) A Matriz Sensibilidade TDEM leva semanas para rodar, quanto maior o intervalo de tempo TDEM, maior o tempo para rodar. No caso de Taubaté foram 10 dias.

d) As mesmas edições de dados no IX devem ser aplicadas para todas sondagens do CTDEM, obtendo-se os mesmos intervalos de tempo e, consequentemente, o mesmo número de pontos.

e) São dois modelos iniciais:

1. Para o cálculo das derivadas parciais na obtenção das matrizes sensibilidade: homogêneo 30 Ohm.m; 2. Para a Inversão Conjunta 2D, com base nos resultados das inversões 1D e/ou nas informações sobre geologia da área de estudo. No caso de Taubaté: $100 \mathrm{Ohm} . \mathrm{m}$ até $40 \mathrm{~m}$ de profundidade (camada rasa resistiva) e $10 \mathrm{Ohm} . \mathrm{m}$ abaixo de $40 \mathrm{~m}$ de profundidade (camada mais profunda condutiva).

f) O valor do lambda é obtido por tentativa e erro, no caso de Taubaté o melhor valor foi 5.

g) Todos os exemplos do tutorial são para os dados de Taubaté. 


\section{Preparação dos dados}

Os dados brutos adquiridos em campo precisam ser preparados para serem inseridos na rotina de inversão conjunta $2 \mathrm{D}$. No caso do dado de $\mathrm{CE}$, nenhuma edição é necessária, apenas precisam ser organizados. Já no caso do CTDEM, é necessário utilizar programa IX1D (Interpex) para remover os pontos ruidosos e converter os dados para resistividade aparente em função do tempo.

A seguir são apresentados os detalhes da preparação dos de CE e CTDEM.

\subsection{Dados de CE}

Os parâmetros de aquisição do CE em Taubaté foram:

- Arranjo: polo-dipolo

- Espaçamento entre eletrodos: $20 \mathrm{~m}$

- Níveis de investigação: 6

- Extensão: 400 m

O equipamento utilizado foi o Syscal Pro (Iris Instruments) e esse é o dado bruto:

\begin{tabular}{|c|c|c|c|c|c|c|}
\hline \multicolumn{7}{|c|}{11} \\
\hline e of & \multicolumn{6}{|c|}{ 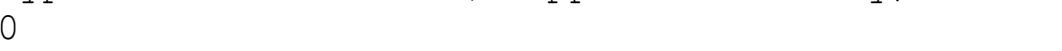 } \\
\hline \multicolumn{7}{|c|}{105} \\
\hline \multicolumn{7}{|c|}{1} \\
\hline \multicolumn{7}{|c|}{ 皮 } \\
\hline 3 & 0.00 & 0.002 & 20.00 & $0.00 \quad 4$ & $10.00 \quad 0$ & .00166 .60 \\
\hline 3 & 0.00 & 0.00 & 40.00 & 0.006 & $50.00 \quad 0$ & $.00 \quad 113.07$ \\
\hline 3 & 0.00 & 0.00 & 60.00 & 0.008 & $30.00 \quad 0$ & $.00 \quad 56.42$ \\
\hline 3 & 0.00 & 0.00 & 80.00 & 0.001 & 00.00 & $0.00 \quad 43.08$ \\
\hline 3 & 0.00 & 0.001 & 100.00 & 0.00 & 120.00 & $0.00 \quad 25.87$ \\
\hline 3 & 0.00 & 0.001 & 120.00 & 0.00 & 140.00 & $0.00 \quad 23.50$ \\
\hline 3 & 20.00 & 0.00 & 40.00 & 0.00 & 60.00 & $0.00 \quad 174.44$ \\
\hline 3 & 20.00 & 0.00 & 60.00 & 0.00 & 80.00 & $0.00 \quad 94.08$ \\
\hline 3 & 20.00 & 0.00 & 80.00 & 0.00 & 100.00 & $0.00 \quad 66.18$ \\
\hline 3 & 20.00 & 0.00 & 100.00 & 0.00 & 120.00 & $0.00 \quad 35.49$ \\
\hline 3 & 20.00 & 0.00 & 120.00 & 0.00 & 140.00 & $0.00 \quad 28.81$ \\
\hline 3 & 20.00 & 0.00 & 140.00 & 0.00 & 160.00 & 0.0016 .13 \\
\hline 3 & 40.00 & 0.00 & 60.00 & 0.00 & 80.00 & $0.00 \quad 162.90$ \\
\hline 3 & 40.00 & 0.00 & 80.00 & 0.00 & 100.00 & $0.00 \quad 117.63$ \\
\hline 3 & 40.00 & 0.00 & 100.00 & 0.00 & 120.00 & $0.00 \quad 59.04$ \\
\hline 3 & 40.00 & 0.00 & 120.00 & 0.00 & 140.00 & $0.00 \quad 41.11$ \\
\hline & 40.00 & 0.00 & 140.00 & 0.00 & 160.00 & $0.00 \quad 19.52$ \\
\hline
\end{tabular}




\begin{tabular}{|c|c|c|c|c|c|c|c|}
\hline 3 & 40.00 & 0.00 & 160.00 & 0.00 & 180.00 & 0.00 & 19.71 \\
\hline & 60.00 & 0.00 & 80.00 & 0.001 & 100.00 & 0.001 & 61.61 \\
\hline & 60.00 & 0.00 & 100.00 & 0.00 & 120.00 & 0.00 & 97.58 \\
\hline & 60.00 & 0.00 & 120.00 & 0.00 & 140.00 & 0.00 & 62.58 \\
\hline & 60.00 & 0.00 & 140.00 & 0.00 & 160.00 & 0.00 & 27.76 \\
\hline & 60.00 & 0.00 & 160.00 & 0.00 & 180.00 & 0.00 & 26.69 \\
\hline & 60.00 & 0.00 & 180.00 & 0.00 & 200.00 & 0.00 & 20.30 \\
\hline & 80. & 0.00 & 100. & 0.00 & 120.00 & 0.00 & 2.50 \\
\hline & 80. & 0.00 & 120. & 0.00 & 140.00 & 0.00 & 88.87 \\
\hline & 80. & 0.00 & 140.00 & 0.00 & 160.00 & 0.00 & 37.77 \\
\hline & 80.00 & 0.00 & 160.00 & 0.00 & 180.00 & 0.00 & 32.08 \\
\hline & 80.00 & 0.00 & 180.00 & 0.00 & 200.00 & 0.00 & 22.85 \\
\hline & 80.00 & 002 & 200.00 & 0.00 & 220.00 & 0.00 & 20.25 \\
\hline & 100.00 & .00 & 120.00 & 0.00 & 140.00 & 0.00 & 154.32 \\
\hline & 100.00 & 0.00 & 140.00 & 0.00 & 160.00 & 0 . & 71.43 \\
\hline & 100.00 & 0.00 & 160.00 & 0.00 & 180.00 & 0. & 53.74 \\
\hline & 100.00 & 0.00 & 180.00 & 0.00 & 200.00 & 0. & 31.46 \\
\hline & 100.00 & 0.00 & 200.00 & 0.00 & 220.00 & & 25.09 \\
\hline & 100.00 & 0.00 & 220.00 & 0.00 & 240.00 & & 18.57 \\
\hline & 120.00 & 0.00 & 140.00 & 0.00 & 160.00 & & 102.80 \\
\hline & 120.00 & 0.00 & 160.00 & 0.00 & 180.00 & 0.00 & \\
\hline & 120.00 & 0 & 180.00 & 0.00 & 200.00 & & \\
\hline & 120.00 & .00 & .00 & 0.00 & 220.00 & 0.00 & 29.82 \\
\hline & 120.00 & 0.00 & 220.00 & 0.00 & 240.00 & 0.00 & 20.49 \\
\hline & 120.00 & 0.00 & 240.00 & 0.00 & 260.00 & 0.00 & 21.51 \\
\hline & 140.00 & 0.00 & .00 & 0.00 & .00 & 0.00 & 129.75 \\
\hline & 140.00 & 0.00 & .00 & 0.00 & 200.00 & 0.00 & 72.80 \\
\hline & 140.00 & 0.00 & .00 & 0.00 & 220.00 & 0.00 & .99 \\
\hline & 140.00 & .00 & 220.00 & 0.00 & 240.00 & 0.00 & 91 \\
\hline & 140.00 & .00 & 240.00 & 0.00 & 260.00 & 0.00 & 21 \\
\hline & 140.00 & .00 & .00 & 0.00 & 280.00 & 0.00 & 54 \\
\hline & 160.00 & .00 & .00 & 0.00 & 200.00 & 0.00 & 80.29 \\
\hline & 160.00 & .00 & 200.00 & 0.00 & 220.00 & 0.00 & 48.59 \\
\hline & 160.00 & 0.00 & 220.00 & 0.00 & 240.00 & 0.00 & 26.22 \\
\hline & 160.00 & 0.00 & 240.00 & 0.00 & 260.00 & 0.00 & 23.67 \\
\hline & 160.00 & 0.00 & 260.00 & 0.00 & 280.00 & 0.00 & 22.92 \\
\hline & 160.00 & 0.00 & 280.00 & 0.00 & 300.00 & 0.00 & 21.07 \\
\hline & 180.00 & 0.00 & 200.00 & 0.00 & 220.00 & 0.00 & 78.69 \\
\hline & 180.00 & 0.00 & 220.00 & 0.00 & 240.00 & 0.00 & 37.89 \\
\hline & 180.00 & 0.00 & 240.00 & 0.00 & 260.00 & 0.00 & 27.74 \\
\hline & 180.00 & 0.00 & 260.00 & 0.00 & 280.00 & 0.00 & 24.97 \\
\hline & 180.00 & 0.00 & 280.00 & 0.00 & 300.00 & 0.00 & 22.56 \\
\hline & 180.00 & 0.00 & 300.00 & 0.00 & 320.00 & 0.00 & 20.21 \\
\hline & 200.00 & 0.00 & 220.00 & 0.00 & 240.00 & 0.00 & 66.18 \\
\hline & 200.00 & 0.00 & 240.00 & 0.00 & 260.00 & 0.00 & 38.22 \\
\hline & 200.00 & 0.00 & 260.00 & 0.00 & 280.00 & 0.00 & 28.96 \\
\hline & 200.00 & 0.00 & 280.00 & 0.00 & 300.00 & 0.00 & 24.76 \\
\hline & 200.00 & 0.00 & 300.00 & 0.00 & 320.00 & 0.00 & 21.35 \\
\hline 3 & 200.00 & 0.00 & 320.00 & 0.00 & 340.00 & 0.00 & 21.88 \\
\hline & 220.00 & 0.00 & 240.00 & 0.00 & 260.00 & 0.00 & 65.76 \\
\hline & 220.00 & 0.00 & 260.00 & 0.00 & 280.00 & 0.00 & 41.84 \\
\hline & 220.00 & 0.00 & 280.00 & 0.00 & 300.00 & 0.00 & 32.20 \\
\hline & 220.00 & 0.00 & 300.00 & 0.00 & 320.00 & 0.00 & 24.73 \\
\hline & 220.00 & 0.00 & 320.00 & 0.00 & 340.00 & 0.00 & 24.15 \\
\hline & 220.00 & 0.00 & 340.00 & 0.00 & 360.00 & 0.00 & 20.13 \\
\hline
\end{tabular}




\begin{tabular}{|c|c|c|c|c|c|c|}
\hline 240.00 & 0.00 & 260.00 & 0.00 & 280.00 & 0.00 & 58.98 \\
\hline 240.00 & 0.00 & 280.00 & 0.00 & 300.00 & 0.00 & 45.56 \\
\hline 40.00 & 0.00 & 300.00 & 0.00 & 320.00 & 0.00 & 30.02 \\
\hline 10.00 & 0.00 & 320.00 & 0.00 & 340.00 & 0.00 & 26.73 \\
\hline 0.00 & 0.00 & 340.00 & 0.00 & 360.00 & 0.00 & 20.99 \\
\hline 0.00 & 0.00 & 360.00 & 0.00 & 380.00 & 0.00 & 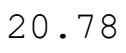 \\
\hline 0.00 & 0.00 & 280.00 & 0.00 & 300.00 & 0.00 & \\
\hline 0.00 & 0.00 & 300.00 & 0.00 & 320.00 & 0.00 & 0.44 \\
\hline 50.00 & 0.00 & 320.00 & 0.00 & 340.00 & 0.00 & . \\
\hline 50.00 & 0.00 & 340.00 & 0.00 & 360.00 & 0.00 & .0 \\
\hline 0.00 & 0.00 & 360.00 & 0.00 & 380.00 & 0.00 & 3.1 \\
\hline 0.00 & 0.00 & 380.00 & 0.00 & 400.00 & 0.00 & 3.4 \\
\hline 30.00 & 0.00 & 300.00 & 0.00 & 320.00 & 0.00 & 55.9 \\
\hline 0.00 & 0.00 & 320.00 & 0.00 & 340.00 & 0.00 & 43.5 \\
\hline 0.00 & 0.00 & 340.00 & 0.00 & 360.00 & 0.00 & 21.0 \\
\hline 0.00 & 0.00 & 360.00 & 0.00 & 380.00 & 0.00 & 3 \\
\hline 0.00 & 0.00 & 380.00 & 0.00 & 400.00 & 0.00 & 22.8 \\
\hline 0.00 & 0.00 & 400.00 & 0.00 & 420.00 & 0.00 & 18.6 \\
\hline 0.00 & 0.00 & 320.00 & 0.00 & 340.00 & 0.00 & 63.4 \\
\hline 0.00 & 0.00 & 340.00 & 0.00 & 360.00 & 0.00 & 8.3 \\
\hline .00 & 0.00 & 360.00 & 0.00 & 380.00 & 0.00 & .4 \\
\hline .00 & 0.00 & 380.00 & 0.00 & 400.00 & 0.00 & - \\
\hline 300.00 & 0.00 & 400.00 & 0.00 & 420.00 & 0.00 & $\angle 1.04$ \\
\hline 320.00 & 0.00 & 340.00 & 0.00 & 360.00 & 0.00 & 62.42 \\
\hline 320.00 & 0.00 & 360.00 & 0.00 & 380.00 & 0.00 & 39.65 \\
\hline 320.00 & 0.00 & 380.00 & 0.00 & 400.00 & 0.00 & $32.9^{\prime}$ \\
\hline 320.00 & 0.00 & 400.00 & 0.00 & 420.00 & 0.00 & 24.5 \\
\hline 340.00 & 0.00 & 360.00 & 0.00 & 380.00 & 0.00 & $5 / .9$ \\
\hline 340.00 & 0.00 & 380.00 & 0.00 & 400.00 & 0.00 & 43.9 \\
\hline 340.00 & 0.00 & 400.00 & 0.00 & 420.00 & 0.00 & $30 \cdot 3^{1}$ \\
\hline 360.00 & 0.00 & 380.00 & 0.00 & 400.00 & 0.00 & 60.5 \\
\hline 360.00 & 0.00 & 400.00 & 0.00 & 420.00 & 0.00 & 39.0 \\
\hline 380.00 & 0.00 & 400.00 & 0.00 & 420.00 & 0.00 & 62.7 \\
\hline
\end{tabular}

As posições dos eletrodos de corrente $(A B / 2)$ e dos eletrodos de potencial $(M N)$ são inseridas nas rotinas de acordo com posições definidas na malha de diferenças finitas, que será explicada no capítulo 4.

Os valores das resistividades aparentes são inseridos no script como um vetor linha.

\subsection{Dados de CTDEM}

Os parâmetros de aquisição do CTDEM em Taubaté foram:

- Arranjo: fixed-loop

- Tamanho do loop transmissor: 200×200 m

- Espaçamento entre sondagens: $25 \mathrm{~m}$ 
- Número de sondagens: 7

- Extensão: $150 \mathrm{~m}$

- Frequências de aquisição: $30 \mathrm{hz}, 7,5 \mathrm{hz}$ e $3 \mathrm{hz}$

O equipamento utilizado foi o PROTEM 57-MK2 (Geonics). O dado bruto é baixado do equipamento no formato .RED. Utiliza-se o programa PROTIX para converter o dado para o formato .USF. Segue um exemplo de dado adquirido no centro do loop:

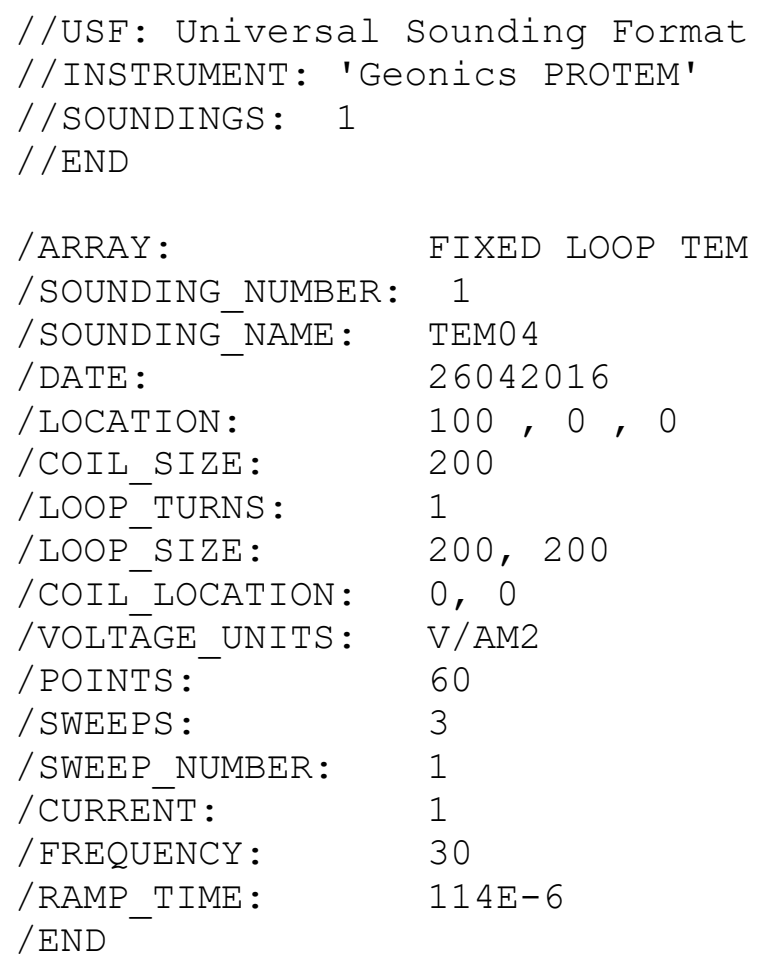




$\begin{array}{llll}19, & 5.5890 \mathrm{E}-03, & 4.83212 \mathrm{E}-09, & 1.32 \mathrm{E}-03 \\ 20, & 7.0920 \mathrm{E}-03, & 2.49617 \mathrm{E}-09, & 1.68 \mathrm{E}-03\end{array}$

$/ E N D$

/SWEEP NUMBER: 2

/CURRENTT: 1

/FREQUENCY: $\quad 7.5$

/RAMP TIME: $\quad 114 \mathrm{E}-6$

$/ \mathrm{END}$

$\begin{array}{rlll}\text { INDEX, } & \text { TIME, } & \text { VOLTAGE, } & \text { WIDTH } \\ 1, & 4.6650 \mathrm{E}-04, & 1.62575 \mathrm{E}-06, & 6.50 \mathrm{E}-05 \\ 2, & 5.4150 \mathrm{E}-04, & 1.20231 \mathrm{E}-06, & 8.50 \mathrm{E}-05 \\ 3, & 6.3900 \mathrm{E}-04, & 8.57622 \mathrm{E}-07, & 1.10 \mathrm{E}-04 \\ 4, & 7.6150 \mathrm{E}-04, & 5.96015 \mathrm{E}-07, & 1.35 \mathrm{E}-04 \\ 5, & 9.1650 \mathrm{E}-04, & 4.03210 \mathrm{E}-07, & 1.75 \mathrm{E}-04 \\ 6, & 1.1170 \mathrm{E}-03, & 2.63629 \mathrm{E}-07, & 2.26 \mathrm{E}-04 \\ 7, & 1.3720 \mathrm{E}-03, & 1.66712 \mathrm{E}-07, & 2.84 \mathrm{E}-04 \\ 8, & 1.6970 \mathrm{E}-03, & 1.02128 \mathrm{E}-07, & 3.66 \mathrm{E}-04 \\ 9, & 2.1120 \mathrm{E}-03, & 6.06987 \mathrm{E}-08, & 4.64 \mathrm{E}-04 \\ 10, & 2.6390 \mathrm{E}-03, & 3.52237 \mathrm{E}-08, & 5.90 \mathrm{E}-04 \\ 11, & 3.3120 \mathrm{E}-03, & 2.00005 \mathrm{E}-08, & 7.56 \mathrm{E}-04 \\ 12, & 4.1690 \mathrm{E}-03, & 1.11785 \mathrm{E}-08, & 9.58 \mathrm{E}-04 \\ 13, & 5.2620 \mathrm{E}-03, & 6.23330 \mathrm{E}-09, & 1.23 \mathrm{E}-03 \\ 14, & 6.6570 \mathrm{E}-03, & 3.49837 \mathrm{E}-09, & 1.56 \mathrm{E}-03 \\ 15, & 8.4370 \mathrm{E}-03, & 2.02096 \mathrm{E}-09, & 2.00 \mathrm{E}-03 \\ 16, & 1.0704 \mathrm{E}-02, & 1.21237 \mathrm{E}-09, & 2.54 \mathrm{E}-03 \\ 17, & 1.3604 \mathrm{E}-02, & 7.35333 \mathrm{E}-10, & 3.26 \mathrm{E}-03 \\ 18, & 1.7304 \mathrm{E}-02, & 4.19439 \mathrm{E}-10, & 4.14 \mathrm{E}-03 \\ 19, & 2.2014 \mathrm{E}-02, & 2.58063 \mathrm{E}-10, & 5.28 \mathrm{E}-03 \\ 20, & 2.8034 \mathrm{E}-02, & 1.36015 \mathrm{E}-10, & 6.76 \mathrm{E}-03\end{array}$

/END

/SWEEP NUMBER: 3

/CURRENTT: 1

/FREQUENCY: 3

/RAMP_TIME: $\quad 114 \mathrm{E}-6$

$/ \mathrm{END}$

$\begin{array}{rccc}\text { INDEX, } & \text { TIME, } & \text { VOLTAGE, } & \text { WIDTH } \\ 1, & 9.9530 \mathrm{E}-04, & 3.38294 \mathrm{E}-07, & 1.63 \mathrm{E}-04 \\ 2, & 1.1830 \mathrm{E}-03, & 2.32108 \mathrm{E}-07, & 2.13 \mathrm{E}-04 \\ 3, & 1.4270 \mathrm{E}-03, & 1.52666 \mathrm{E}-07, & 2.75 \mathrm{E}-04 \\ 4, & 1.7330 \mathrm{E}-03, & 9.72368 \mathrm{E}-08, & 3.37 \mathrm{E}-04 \\ 5, & 2.1200 \mathrm{E}-03, & 6.02758 \mathrm{E}-08, & 4.37 \mathrm{E}-04 \\ 6, & 2.6200 \mathrm{E}-03, & 3.60466 \mathrm{E}-08, & 5.63 \mathrm{E}-04 \\ 7, & 3.2580 \mathrm{E}-03, & 2.10211 \mathrm{E}-08, & 7.13 \mathrm{E}-04 \\ 8, & 4.0700 \mathrm{E}-03, & 1.20357 \mathrm{E}-08, & 9.11 \mathrm{E}-04 \\ 9, & 5.1080 \mathrm{E}-03, & 6.82532 \mathrm{E}-09, & 1.17 \mathrm{E}-03 \\ 10, & 6.4270 \mathrm{E}-03, & 3.91324 \mathrm{E}-09, & 1.47 \mathrm{E}-03 \\ 11, & 8.1080 \mathrm{E}-03, & 2.32303 \mathrm{E}-09, & 1.89 \mathrm{E}-03 \\ 12, & 1.0254 \mathrm{E}-02, & 1.43923 \mathrm{E}-09, & 2.40 \mathrm{E}-03 \\ 13, & 1.2984 \mathrm{E}-02, & 9.22766 \mathrm{E}-10, & 3.06 \mathrm{E}-03\end{array}$




$\begin{array}{llll}14, & 1.6474 \mathrm{E}-02, & 5.72472 \mathrm{E}-10, & 3.92 \mathrm{E}-03 \\ 15, & 2.0924 \mathrm{E}-02, & 3.51323 \mathrm{E}-10, & 4.98 \mathrm{E}-03 \\ 16, & 2.6594 \mathrm{E}-02, & 2.61481 \mathrm{E}-10, & 6.36 \mathrm{E}-03 \\ 17, & 3.3844 \mathrm{E}-02, & 1.54644 \mathrm{E}-10, & 8.14 \mathrm{E}-03 \\ 18, & 4.3084 \mathrm{E}-02, & 1.32014 \mathrm{E}-10, & 1.03 \mathrm{E}-02 \\ 19, & 5.4864 \mathrm{E}-02, & 8.45000 \mathrm{E}-11, & 1.32 \mathrm{E}-02 \\ 20, & 6.9894 \mathrm{E}-02, & 8.02000 \mathrm{E}-11, & 1.68 \mathrm{E}-02\end{array}$

/END

Os dados do CTDEM são então carregados no programa IX1D para que sejam excluídos os pontos ruidosos e então são exportados na forma de resistividade aparente em função do tempo (ASCII Data File). Para maiores detalhes sobre o tratamento dos dados no programa IX1D, consultar o trabalho de Campaña (2015).

Dado adquirido no centro do loop do exemplo acima após tratamento:

\begin{tabular}{ccl} 
DATASET: TEMO4 & \multicolumn{1}{c}{0.00} \\
EAST $:$ & NORTH: & 0.00 \\
NUMBER & TIME & RESISTIVITY \\
1 & 0.200600 & 43.179070 \\
2 & 0.250600 & 36.208424 \\
3 & 0.314400 & 30.905462 \\
4 & 0.395600 & 26.713129 \\
5 & 0.499400 & 23.350004 \\
6 & 0.631300 & 20.694447 \\
7 & 0.799400 & 18.622952 \\
8 & 1.014000 & 17.023289 \\
9 & 1.287000 & 15.858989 \\
10 & 1.636000 & 15.059137 \\
11 & 2.081000 & 14.566569 \\
12 & 2.648000 & 14.358798 \\
13 & 3.373000 & 14.402378 \\
14 & 0.352500 & 30.398853 \\
15 & 0.427500 & 26.950863 \\
16 & 0.525000 & 23.969507 \\
17 & 0.647500 & 21.536919 \\
18 & 0.802500 & 19.548037 \\
19 & 1.003000 & 17.884960 \\
20 & 1.258000 & 16.642570 \\
21 & 1.583000 & 15.730442 \\
22 & 1.998000 & 15.094361 \\
23 & 2.525000 & 14.689892 \\
24 & 3.198000 & 14.451911 \\
25 & 4.055000 & 14.354246 \\
26 & 5.148000 & 14.256063 \\
27 & 0.881300 & 18.780180 \\
28 & 1.069000 & 17.500235 \\
29 & 1.313000 & 16.424631 \\
30 & 1.619000 & 15.647984 \\
31 & 2.006000 & 15.054638 \\
32 & 2.506000 & 14.621095 \\
& &
\end{tabular}




$\begin{array}{rrr}33 & 3.144000 & 14.360596 \\ 34 & 3.956000 & 14.200397 \\ 35 & 4.994000 & 14.080110 \\ 36 & 6.313000 & 13.847968 \\ 37 & 7.994000 & 13.181976 \\ 38 & 10.140000 & 12.340966\end{array}$

\section{Definição da malha de diferenças finitas}

A mesma malha de diferença finitas é utilizada para ambos os dados de CE e CTDEM. A Figura 1 abaixo apresenta um exemplo da malha utilizada para os dados de Taubaté e a Figura 2 apresenta um zoom da mesma malha.

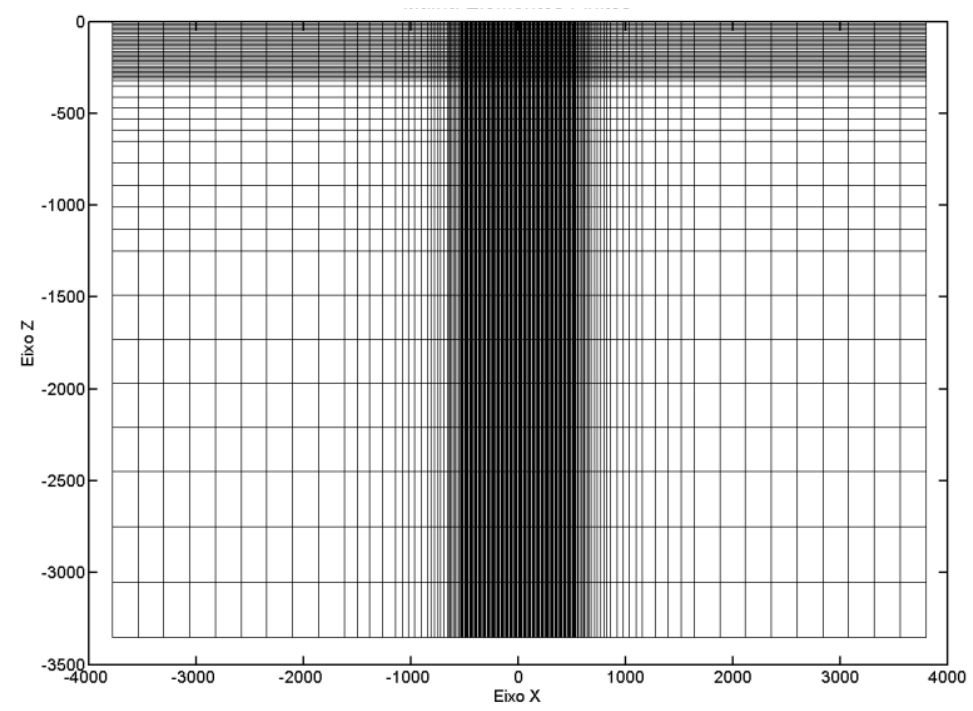

Figura 1. Malha das diferenças finitas utilizada para os dados de Taubaté (Bortolozo, 2016).

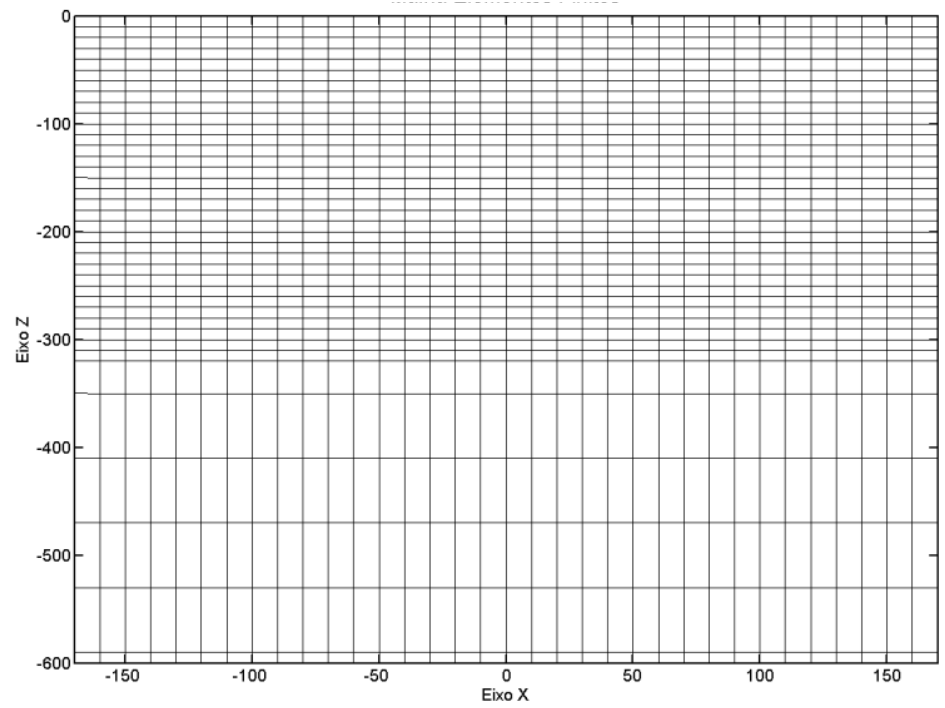

Figura 2. Detalhe da malha utilizada para os dados de Taubaté (Bortolozo, 2016). 
Os parâmetros que definem a malha são:

a) $\mathbf{d x}$ e $\mathbf{d z}$ representam os espaçamentos entre nós nas direções horizontal ( $x$ ) e vertical (z), respectivamente. No caso de Taubaté foram utilizados os seguintes valores:

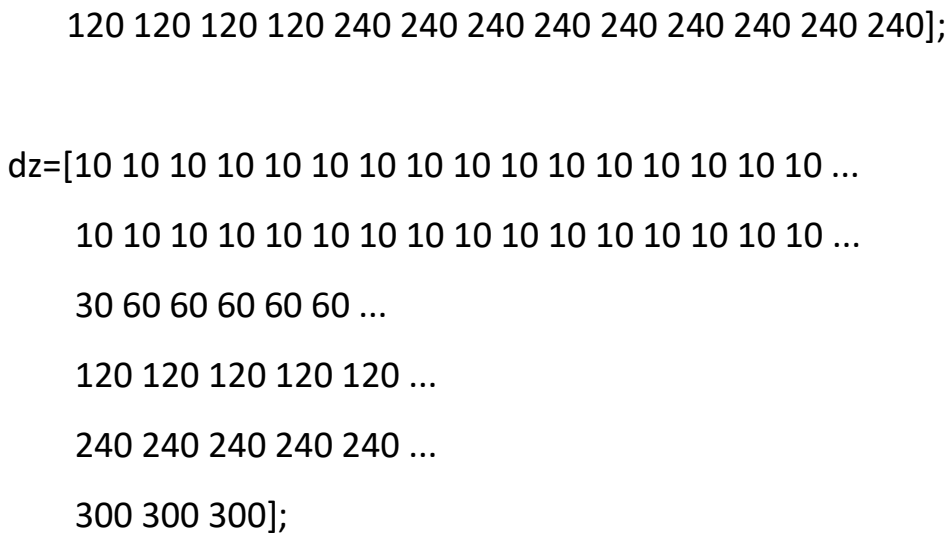

b) $\mathbf{x}$ e $\mathbf{z}$ representam as posições de cada nó. No caso de Taubaté temos:

- $\quad$ v varia de -3780 a $3800 \mathrm{~m}$, sendo 0 a posição central.

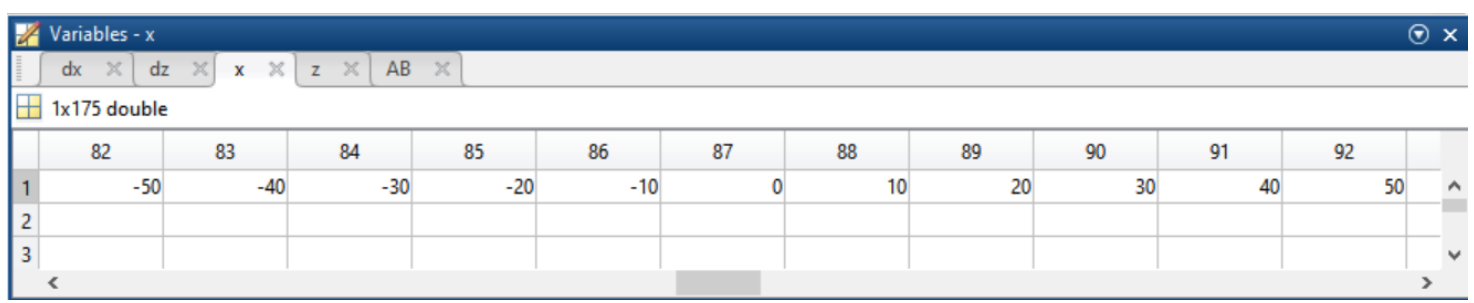

- $\quad$ z varia de 0 (superfície) a $3350 \mathrm{~m}$ de profundidade.

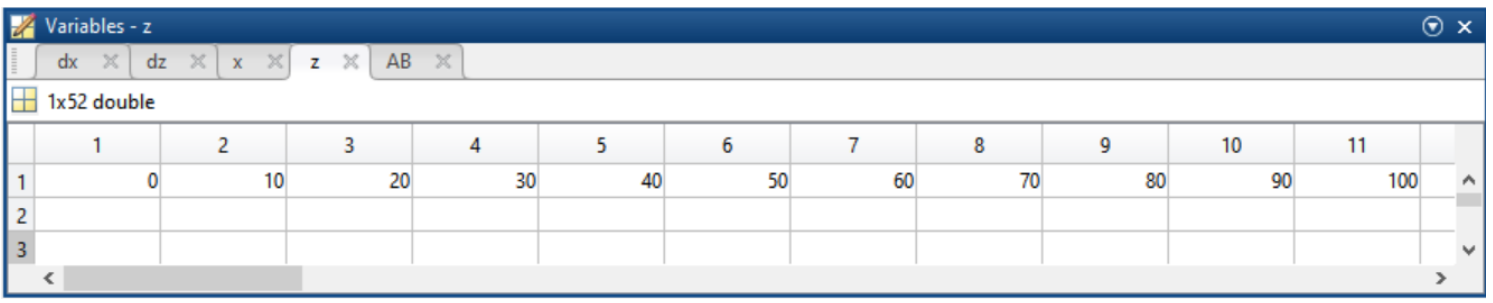


As posições dos eletrodos do CE e as posições das sondagens do CTDEM são inseridos nas rotinas de acordo as posições definidas na malha.

\section{Matriz Sensibilidade}

A Matriz Sensibilidade representa as derivadas parciais que são calculadas para um meio homogêneo pelo método das diferenças finitas uma vez antes do processo iterativo, formando o primeiro conjunto de derivadas do algoritmo. É necessário calcular a Matriz Sensibilidade tanto para os dados de CE quanto para os dados de CTDEM.

A seguir são apresentados os detalhes de como implementar as rotinas da Matriz Sensibilidade para ambos os dados.

\subsection{Rotina da Matriz Sensibilidade CE}

a) Posições dos eletrodos de corrente AB/2 na malha, A (- 200 a 180 m), B no 'infinito':

$A B=[67 ; 69 ; 71 ; 73 ; 75 ; 77 ; 79 ; 81 ; 83 ; 85 ; 87 ; 89 ; 91 ; 93 ; 95 ; 97 ; 99 ; 101 ; 103 ; 105]$;

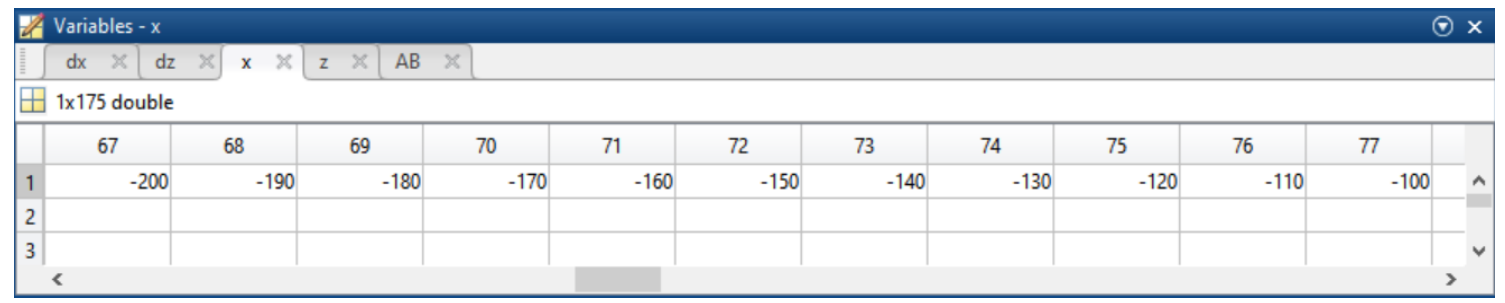

b) Posições dos eletrodos de eletrodos de potencial MN na malha $(-180-160 ;-160-140 ; \ldots$; 180 200; 200 220):

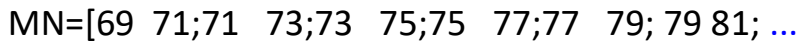

$$
\begin{aligned}
& \begin{array}{lllllll}
71 & 73 ; 73 & 75 ; 75 & 77 ; 77 & 79 ; 79 & 81 ; 81 & 83 ; \ldots
\end{array} \\
& \begin{array}{lllllll}
73 & 75 ; 75 & 77 ; 77 & 79 ; 79 & 81 ; 81 & 83 ; 83 & 85 ; \ldots
\end{array} \\
& \begin{array}{lllllll}
75 & 77 ; 77 & 79 ; 79 & 81 ; 81 & 83 ; 83 & 85 ; 85 & 87 ; \ldots
\end{array} \\
& \begin{array}{lllllll}
77 & 79 ; 79 & 81 ; 8 & 83 ; 83 & 85 ; 85 & 87 ; 87 & 89 ; \ldots
\end{array} \\
& 79 \begin{array}{lllllll}
81 ; 81 & 83 ; 83 & 85 ; 85 & 87 ; 87 & 89 ; 89 & 91 ; \ldots
\end{array} \\
& \begin{array}{llllllll}
81 & 83 ; 83 & 85 ; 85 & 87 ; 87 & 89 ; 89 & 91 ; 91 & 93 ; \ldots
\end{array} \\
& \begin{array}{llllllll}
83 & 85 ; 85 & 87 ; 87 & 89 ; 89 & 91 ; 91 & 93 ; 93 & 95 ; \ldots
\end{array} \\
& \begin{array}{lllllll}
85 & 87 ; 87 & 89 ; 89 & 91 ; 91 & 93 ; 93 & 95 ; 95 & 97 ; \ldots
\end{array}
\end{aligned}
$$




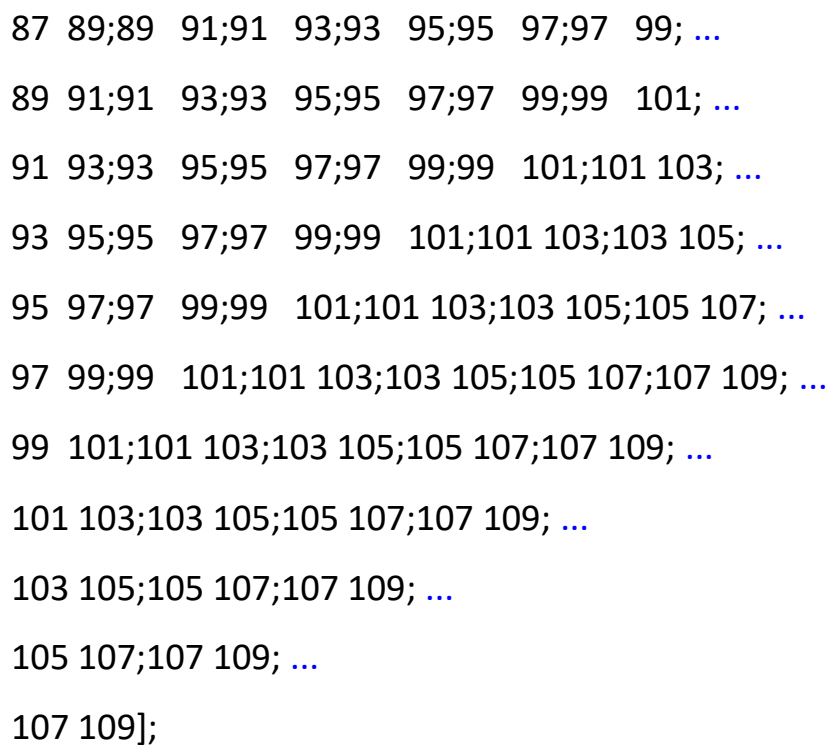

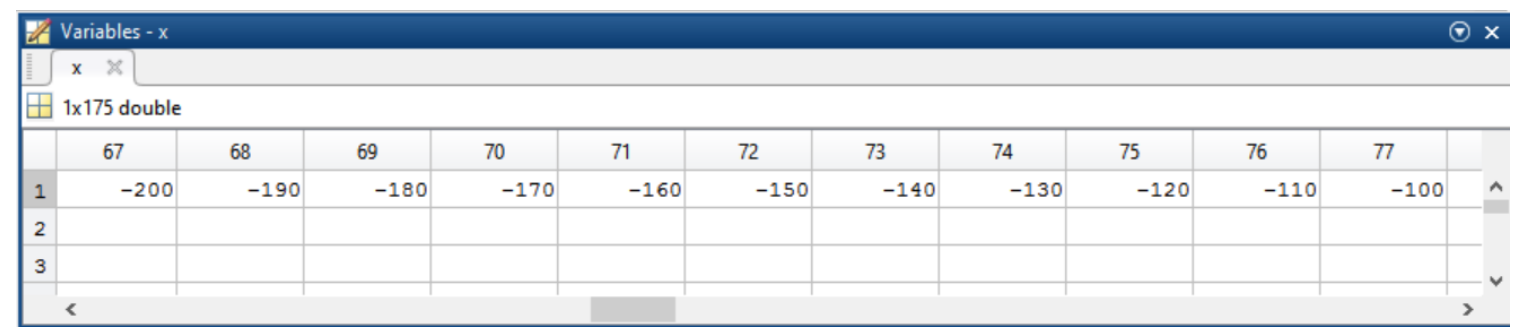

c) Níveis de investigação (6):

* Parâmetros difabs para o fechamento do arranjo

difabs $=6$ *nes(1,length( $A B(:, 1)))$;

$\operatorname{difabs}($ end) $=1$;

difabs(end-1)=2;

$\operatorname{difabs}($ end-2)=3;

$\operatorname{difabs}($ end-3) $=4$;

$\operatorname{difabs}($ end-4)=5;

d) Modelo inicial (homogênio 30 Ohm.m):

rcel=ones((length(z)), (length(x)-1));

rcel=rcel*30;

e) Cálculo da resistividade aparente teórica (modelagem direta):

[rhoap_final_s] = mod_DC_FD_v3(dx, dz, x, z, rcel, numabs, AB, MN); 
f) Cálculo da Matriz Sensibilidade CE:

[Matriz_sensibilidade_CE]=del_mod_DC_v2(rcel, MN, dx, dz, x, z,numabs, AB, rhoap_final_s);

save('Matriz_Sensibilidade_CE.mat', 'Matriz_sensibilidade_CE');

\subsection{Rotina da Matriz Sensibilidade CTDEM}

a) Tempos em milissegundos após tratamento dos dados no programa IX1D, conforme explicado na seção 3.2 .

$$
\begin{array}{r}
\text { tempos } 2=[0.20060 \\
0.25060 \\
0.31440 \\
0.39560 \\
0.49940 \\
0.63130 \\
0.79940 \\
1.01400 \\
1.28700 \\
1.63600 \\
2.08100 \\
2.64800 \\
3.37300 \\
0.35250 \\
0.42750 \\
0.52500 \\
0.64750 \\
0.80250 \\
1.00300 \\
1.25800 \\
1.58300 \\
1.99800 \\
2.52500 \\
3.19800 \\
4.05500 \\
\end{array}
$$


5.14800

0.88130

1.06900

1.31300

1.61900

2.00600

2.50600

3.14400

3.95600

4.99400

6.31300

7.99400

$10.14000]$

b) Conversão para segundos:

tempos=tempos $2 / 1000$;

c) Ordenação dos tempos em ordem crescente:

tempos=sort(tempos);

d) Área efetiva da bobina 3D $\left(200 \mathrm{~m}^{2}\right)$ :

area_efetiv $=200$;

e) Posições das sondagens ( $25 \mathrm{~m}$ e $75 \mathrm{~m}$ arredondados por causa da malha): poisicao_de_med=[-80 -50 -30 03050 80];

f) Posições dos fios na direção $x$ :

posicao_de_fio=[-100 100];

g) Modelo inicial (homogênio 30 Ohm.m):

rcel=ones((length(z)), (length(x)-1));

rcel=rcel*30;

h) Cálculo da resistividade aparente teórica (modelagem direta): 
tic

[ARcal fBS]=Mod_tdem_v8(nSite,t0max,tini,nob,tob,ncwtot,rcel,x,z,nw,nc,nL,ncSite,dz,dx,nc0);

toc

$[\mathrm{rr}, \mathrm{cc}, \mathrm{vv}]=$ find $(\mathrm{ARcal}>0$ \& ARcal $<=4000) ; \%$ para excluir valores anômalos

i) Cálculo da Matriz Sensibilidade TDEM:

[Matriz_sensibilidade_CTDEM] =del_mod_tdem_v2(rcel,dx,dz,x,z,rhoap_final',nSite,tOmax,tini,nob,to b, ncwtot, nw, dxw, nc,nL,ncSite);

save('Matriz_Sensibilidade_CTDEM.mat', 'Matriz_sensibilidade_CTDEM');

\section{Rotina de Inversão Conjunta 2D CE/CTDEM}

a) Modelo Inicial:

rcel=ones((length(z)), (length(x)-1));

rcel=rcel*100; $\quad \% 100 \mathrm{Ohm} . \mathrm{m}$ até $40 \mathrm{~m}$ de profundidade

rcel(5:end,1:end $)=10 ; \quad \% 10$ Ohm.m abaixo de $40 \mathrm{~m}$ de profundidade

b) Dados de resistividade aparente para as sondagens do CTDEM após tratamento dos dados no programa IX1D, conforme explicado na seção 3.2. No caso de Taubaté foram 7 sondagens, portanto são 7 colunas:

\begin{tabular}{|c|c|c|c|c|c|c|}
\hline [57.812988 & 48.984051 & 44.563042 & 43.179070 & 43.940285 & 47.369347 & 55.528801 \\
\hline 47.867382 & 41.054188 & 37.441349 & 36.208424 & 36.927029 & 39.768330 & 46.165321; \\
\hline 39.978558 & 34.767708 & 31.899797 & 30.905462 & 31.485380 & 33.735455 & 38.700645; \\
\hline 33.707546 & 29.749146 & 27.509485 & 26.713129 & 27.179976 & 28.923378 & 32.746311; \\
\hline 28.722433 & 25.735409 & 23.979328 & 23.350004 & 23.721405 & 25.070808 & 28.001520; \\
\hline 24.789337 & 22.543737 & 21.185568 & 20.694447 & 20.991949 & 22.028902 & 24.270834; \\
\hline 21.713133 & 20.051445 & 19.005241 & 18.622952 & 18.866714 & 19.663391 & 21.381800; \\
\hline 19.345419 & 18.107643 & 17.305368 & 17.023289 & 17.220814 & 17.826870 & 19.157171; \\
\hline 17.605425 & 16.687462 & 16.069307 & 15.858989 & 16.024508 & 16.487152 & 17.528059; \\
\hline 16.381628 & 15.697648 & 15.216442 & 15.059137 & 15.198894 & 15.549941 & 16.375994; \\
\hline 15.579860 & 15.064428 & 14.686668 & 14.566569 & 14.684717 & 14.942637 & 15.611696; \\
\hline 15.155268 & 14.763847 & 14.453513 & 14.358798 & 14.458323 & 14.641261 & 15.193277; \\
\hline 15.043389 & 14.743845 & 14.482067 & 14.402378 & 14.485219 & 14.602140 & 15.067096; \\
\hline 38.623363 & 33.827145 & 31.301588 & 30.398853 & 30.779385 & 33.041134 & 37.51129 \\
\hline
\end{tabular}




\begin{abstract}
$\begin{array}{lllllll}33.560604 & 29.751131 & 27.696447 & 26.950863 & 27.260958 & 29.089926 & 32.683083 \text {; }\end{array}$
$\begin{array}{lllllll}29.163206 & 26.211155 & 24.571186 & 23.969507 & 24.213140 & 25.674179 & 28.497890 \text {; }\end{array}$

$\begin{array}{lllllll}25.590080 & 23.307400 & 22.012060 & 21.536919 & 21.726130 & 22.891041 & 25.110254 \text {; }\end{array}$

$\begin{array}{lllllll}22.648724 & 20.923065 & 19.914759 & 19.548037 & 19.693430 & 20.613649 & 22.336121 ;\end{array}$

$\begin{array}{llllllll}20.259510 & 18.937794 & 18.164095 & 17.884960 & 18.004265 & 18.725718 & 20.076075 ;\end{array}$

$\begin{array}{llllllll}18.435738 & 17.441181 & 16.849449 & 16.642570 & 16.734447 & 17.304937 & 18.361469 ;\end{array}$

$\begin{array}{llllllll}17.087584 & 16.333868 & 15.882366 & 15.730442 & 15.803612 & 16.248117 & 17.086149 ;\end{array}$

$\begin{array}{lllllll}16.121803 & 15.554213 & 15.207089 & 15.094361 & 15.149503 & 15.491279 & 16.155359 ;\end{array}$

$\begin{array}{llllllll}15.470703 & 15.039488 & 14.779482 & 14.689892 & 14.728137 & 14.978156 & 15.518961 ;\end{array}$

$\begin{array}{llllllll}15.060301 & 14.721707 & 14.521669 & 14.451911 & 14.472896 & 14.647241 & 15.091452 ;\end{array}$

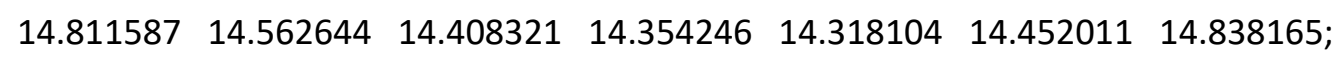

$\begin{array}{lllllll}14.624340 & 14.426450 & 14.294676 & 14.256063 & 14.157258 & 14.292901 & 14.637398] ;\end{array}$
\end{abstract}

c) Dados de resistividade aparente do CE (vetor linha):

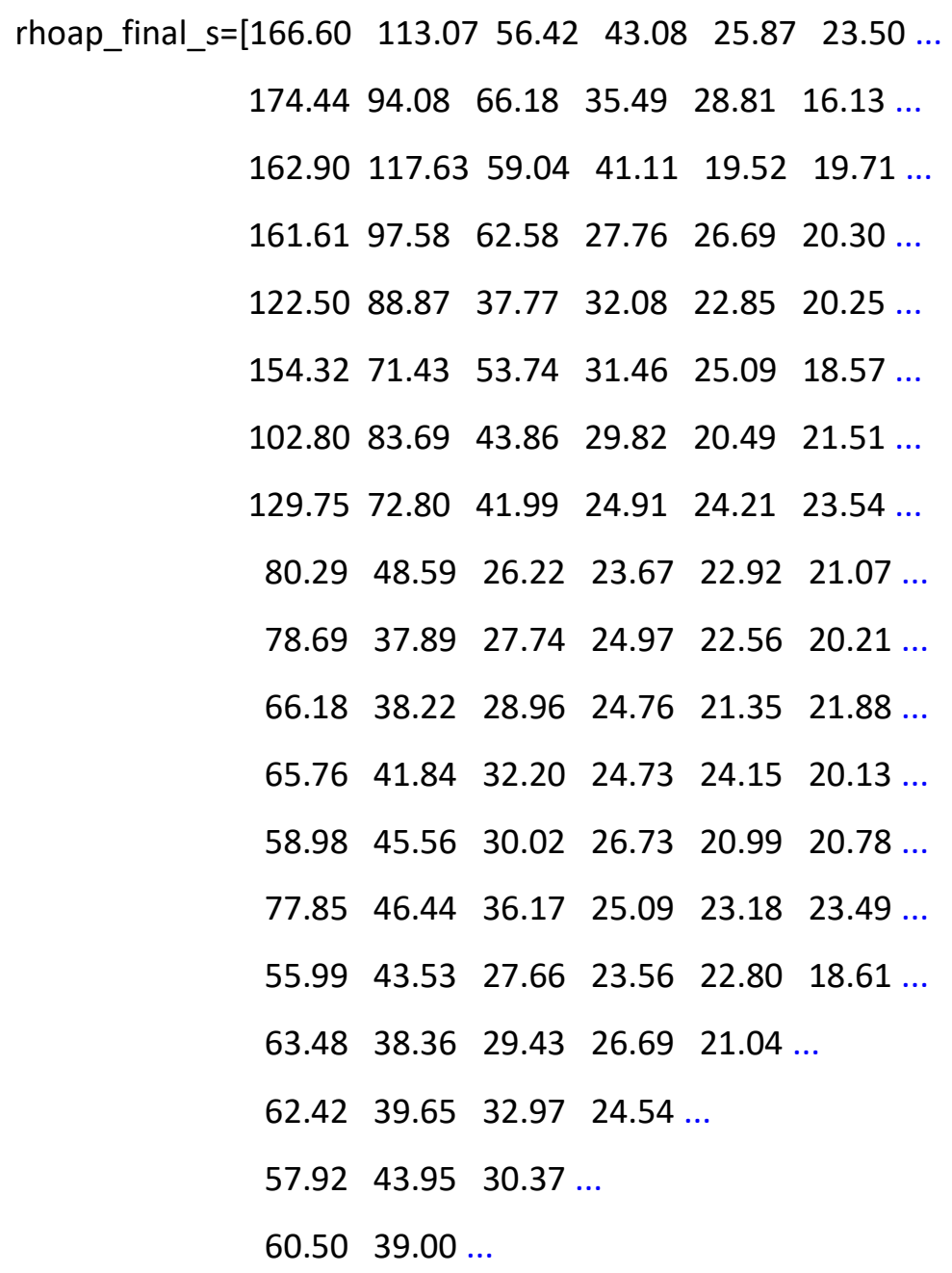


62.74];

d) Matriz Sensibilidade CE:

load('Matriz_Sensibilidade_CE.mat');

A1 = Matriz_sensibilidade_CE;

e) Matriz Sensibilidade CTDEM:

load('Matriz_Sensibilidade_CTDEM.mat');

A2 = Matriz_sensibilidade_CTDEM;

f) Lambda: deve ser testado valores entre 0.01 até 100 .

g) Número de iterações: while continv< $<=30$

h) Erro: if error_it $>1.410 *$ error_pre

O programa para de rodar se o erro da iteração atual (error_it) é maior que 1.41 o valor do erro da iteração anterior (error_pre). O valor de 1.41 pode ser modificado de acordo com as necessidades do dado, pois observamos que a razão arror_it/error_pre é maior inicialmente e tende a diminuir com o aumento do número de iterações.

\section{Bibliografia}

Bortolozo, C.A., 2016. Inversão conjunta 1D e 2D de dados de Eletrorresistividade e TDEM aplicados em estudos de hidrogeologia na bacia do Paraná. Tese de Doutorado (Geofísica), IAG-USP.

Bortolozo, C.A., Campaña, J.D.R., Junior, M.A.C., Porsani, J.L. and dos Santos, F.A.M., 2016. The Effects of Negative Values of Apparent Resistivity in TEM Surveys. International Journal of Geosciences, 7, 1182-1190.

Campaña, J.D.R., 2015. Análise do campo magnético secundário 3D usado no método TDEM: Aplicações em aquíferos fraturados. Dissertação de Mestrado (Geofísica), IAG-USP.

Dey, A., Morrison, F., 1979. Resistivity modeling for arbitrarily shaped two-dimensional structures. Geophysical Prospecting, vol. 27, 106-136. 
Geonics, 1998. PROTEM-57MK2, operating manual. Geonics Limited, Mississauga, Ontario, Canada.

McNeill, J.D., 1994. Principles and application of time domain electromagnetic techniques for resistivity sounding, Technical Note TN-27, Geonics Ltd, Mississauga, Ontario, Canada.

Oristaglio, M. L. e Hohmann, G. W., 1984. Diffusion of electromagnetic fields into a two-dimensional earth: A finite-difference approach. Geophysics, 49, 870-894.

Sasaki, Y., 1989. Two-dimensional joint inversion of magnetotelluric and dipole-dipole resistivity data. Geophysics, 54, 254-262.

Telford, W.M.; Geldart, L.P.; Sheriff, R.E., 1990. Applied Geophysics, Cambridge University Press, 2aㅡ edição. 